\title{
Scalable Synthesis of Functionalized Ferrocenyl Azides and Amines Enabled by Flow Chemistry
}

Merlin Kleoff, Johannes Schwan, Lisa Boeser, Bence Hartmayer, Mathias Christmann,* Biprajit Sarkar, ${ }^{*}$ and Philipp Heretsch*

\section{Supporting Information}

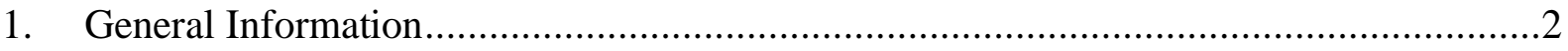

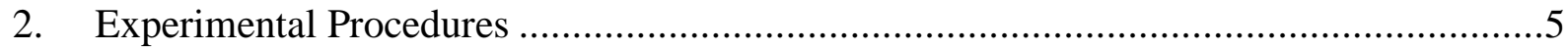

3. NMR Spectra of Synthesized Compounds............................................................52

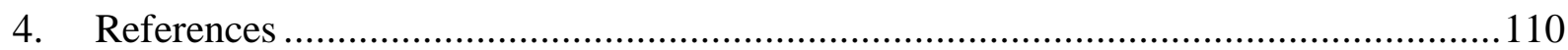




\section{General Information}

\subsection{Materials and Methods}

Unless otherwise noted, all reactions and workups were performed open to air. All substances sensitive to water and oxygen were handled under an argon atmosphere using standard Schlenk techniques and oil pump vacuum. Room temperature (rt) refers to $25^{\circ} \mathrm{C}$.

Anhydrous THF was distilled under an atmosphere of argon over sodium/benzophenone and stored over activated $3 \AA$ mol sieves. Anhydrous $n$-hexane was obtained by storing over activated $3 \AA$ mol sieves for $48 \mathrm{~h}$. Anhydrous $\mathrm{CH}_{2} \mathrm{Cl}_{2}, \mathrm{MeCN}$, and $n$-pentane were obtained from a solvent system MB SPS- 800 from the company MBRAUN. Anhydrous $\mathrm{HN} i P r_{2}$ and $n$-BuOH were distilled from $\mathrm{KOH}$ and stored over activated $3 \AA$ mol sieves. TMEDA was distilled from $\mathrm{CaH}_{2}$ and stored over activated $3 \AA$ mol sieves in the dark at $-18^{\circ} \mathrm{C}$.

EtOAc, $n$-pentane and $n$-hexane were purified by distillation on a rotary evaporator. All other solvents and commercially available reagents were used without further purification unless otherwise stated.

If necessary, $n$ - $\mathrm{BuLi}$ was titrated before use in $\mathrm{Et}_{2} \mathrm{O}$ with $n$ - $\mathrm{BuOH}$ and 2,2'-bipyridine as indicator.

$3 \AA$ mol sieves were activated by drying over night at $100{ }^{\circ} \mathrm{C}$ in a drying cabinet followed by drying at $10^{-3}$ mbar for $30 \mathrm{~min}$ at $600{ }^{\circ} \mathrm{C} . \mathrm{ZnCl}_{2}$ was dried at $10^{-3}$ mbar for at least $15 \mathrm{~min}$ at $600{ }^{\circ} \mathrm{C} . \mathrm{ZnCl}_{2}$ solutions in THF were stored over $3 \AA$ mol sieves. Tosyl azide was stored as a solution in THF over $\mathrm{CaH}_{2}$ (for the preparation, see below).

The following compounds were prepared according to the literature: 2,4,6-Triisopropylbenzenesulfonyl azide $^{[1]}$ and para-chlorobenzenesulfonyl azide. ${ }^{[2]}$

Medium pressure liquid chromatography (MPLC) was performed with a TELEDYNE ISCO CombiFlash Rf or a TELEDYNE ISCO Combi-Flash Rf200 using prepacked $\mathrm{SiO}_{2}$-columns and cartridges from TELEDYNE. UV response was monitored at $254 \mathrm{~nm}$ and $280 \mathrm{~nm}$. As eluents, cyclohexane (99.5\% + quality) and EtOAc (HPLC grade) were used.

High performance liquid chromatography (HPLC) was conducted on a modular KNAUER HPLC system with a UV detector at $254 \mathrm{~nm}$ and differential refractometer on a $4 \times 250 \mathrm{~mm}$ column packed with Nucleosil 50-5 from MACHERY-NAGEL.

For column chromatography, silica $60 \mathrm{M}(0.040-0.063 \mathrm{~mm})$ from MACHERY-NAGEL or basic Aluminum oxide from ACROS or MACHERY-NAGEL were used. Basic aluminum oxide was deactivated prior use with $5 \mathrm{wt} \%$ water by adding the calculated amount of water to aluminum oxide followed by vigorously shaking for some minutes. Alternatively, after addition of water, the aluminum oxide was rotated at atmospheric pressure on a rotary evaporator for $15 \mathrm{~min}$. 
Unless otherwise stated, concentration under reduced pressure was performed by rotary evaporation at $40{ }^{\circ} \mathrm{C}$ and the appropriate pressure.

All reactions involving ferrocenyl azides were performed with additional care on a scale of less than $1 \mathrm{~g}$ and under modest exclusion of light. Concentration of solutions of ferrocenyl azides was performed by rotary evaporation at $38{ }^{\circ} \mathrm{C}$ and appropriate pressure. All ferrocenyl azides were stored as solids or oils, respectively, in the dark at $4{ }^{\circ} \mathrm{C}$. When stored cold, most ferrocenyl azides were found to be relatively stable and decomposed very slowly over months. The decomposition products can easily be removed by filtration of a concentrated solution of the ferrocenyl azide in $\mathrm{CH}_{2} \mathrm{Cl}_{2}$ over a pad of basic $\mathrm{Al}_{2} \mathrm{O}_{3}$ (deactivated with $5 \mathrm{wt} \% \mathrm{H}_{2} \mathrm{O}$ ). In solution, ferrocenyl azides decompose within a few days. Therefore, samples for $\mathrm{NMR}$ spectra in $\mathrm{CDCl}_{3}$ were measured within minutes to hours after preparation.

\subsection{Analysis}

Reaction monitoring: Reactions were monitored by TLC carried out on Merck Silica Gel $50 \mathrm{~F}_{254}$-plates and visualized by fluorescence quenching under UV-light $(\lambda=254 \mathrm{~nm})$ or by using a stain of vanillin (6 g vanillin, $1.5 \mathrm{~mL} 96 \% \mathrm{H}_{2} \mathrm{SO}_{4}, 100 \mathrm{~mL} \mathrm{EtOH}$ ) and heat as developing agent.

NMR spectroscopy: All NMR spectra were acquired on a JEOL ECZ400 (400 MHz), a JEOL ECS400 (400 MHz), a JEOL ECX $400(400 \mathrm{MHz})$, a JEOL ECP $500(500 \mathrm{MHz})$, a Bruker Avance 500 $(500 \mathrm{MHz})$, a Varian INOVA $600(600 \mathrm{MHz})$ or a Bruker Avance $700(700 \mathrm{MHz})$ in the reported deuterated solvents. Chemical shifts are reported in parts per million (ppm) with reference to the residual solvent peaks. The given multiplicities are phenomenological, thus, the actual appearance of the signals is stated and not the theoretically expected one. The following abbreviations were used to designate multiplicities: $\mathrm{s}=$ singlet, $\mathrm{d}=$ doublet, $\mathrm{t}=$ triplet, $\mathrm{q}=$ quartet, quint $=$ quintet. In case no multiplicity could be identified, the chemical shift range of the signal is given $(\mathrm{m}=$ multiplet $)$.

Infrared spectroscopy: Infrared (IR) spectra were measured on a NICOLET Nexus 670/870 FT-IR spectrometer or a JASCO FT/IR-4100 spectrometer. Wavenumbers $\tilde{v}$ are given in $\mathrm{cm}^{-1}$ and intensities are as follows: $\mathrm{s}=$ strong, $\mathrm{m}=$ medium, $\mathrm{w}=$ weak .

High resolution mass spectrometry: High-resolution mass spectra (HRMS) were recorded using an AGILENT 6210 ESI-TOF spectrometer.

Optical Rotation: Optical rotations were measured on a JASCO P-2000 polarimeter at $589 \mathrm{~nm}$ using $100 \mathrm{~mm}$ cells and the solvent and concentration $(\mathrm{g} / 100 \mathrm{~mL})$ indicated. 


\subsection{Flow Equipment}

All flow chemistry experiments were carried out using the following equipment:

Tubings, connectors and valves: FEP tubing (outer diameter 1/16", inner diameter 1/32”) and PTFE tubing (outer diameter 1/8", inner diameter 1/16") were obtained from BOLA. T-mixers made from stainless steel $316 \mathrm{~L}$ were provided by VICI. A static mixer with an internal volume of $2.2 \mu \mathrm{L}$ made from PEEK/UHMWPE was provided by UPCHURCH SCIENTIFIC. Tubings and mixers were connected using either coned 10-32 UNF fittings made from stainless steel 316L obtained from UPCHURCH SCIENTIFIC or flat bottom $1 / 4-28 \mathrm{UNF}$ gripper fittings made from PP obtained from DIBAFIT. Adapters for 1/4-28 UNF systems were made from PP or PTFE and were provided from UPCHURCH SCIENTIFIC. Manual 6-way-valves made from stainless steel 316L were provided by KNAUER.

Gas delivery: A controlled stream of argon gas was delivered using a mass flow controller (EL-FLOW Prestige Series) from BRONKHORST. Connectors and adapters were provided from SWAGELOK.

Pumping systems: Liquids were delivered using 3D-printed syringe pumps based on a setup developed by M. Spano from the group of M. Croatt. ${ }^{[3]}$ Gas-tight Luer-Lock syringes made from glass were obtained from VWR. For solvent supply, a Waters 515 HPLC pump was used.

\section{General remarks for performing flow experiments involving $\boldsymbol{n}$-butyllithium:}

Prior use, tubes were flushed with dry THF for at least 3 residence times. After performing reactions involving $n$-butyllithium, the tubes were washed with $i \mathrm{PrOH}$ (HPLC grade) for at least 3 residence times. Any blockages arising from solid formation were cleared using a solution of $\mathrm{MeOH} / \mathrm{H}_{2} \mathrm{O} / \mathrm{AcOH}$ (10:1:1), followed by washing with $i \mathrm{PrOH}$ (HPLC grade) for at least 3 residence times. 


\section{Experimental Procedures}

\subsection{Optimization of Reaction Conditions for the Synthesis of Ferrocenyl} Azides in Batch

Screening of different organolithiums and arylsulfonyl azides in batch
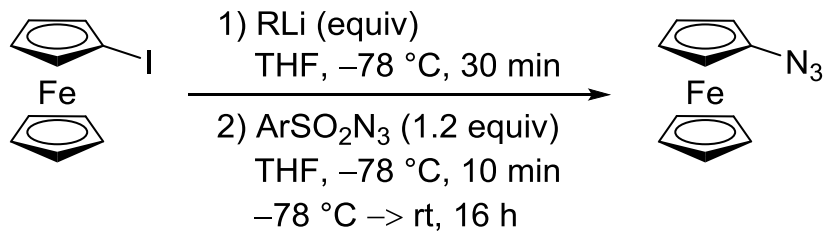

Table S1: Screening of different organolithiums (RLi) and arylsulfonyl azides $\left(\mathrm{ArSO}_{2} \mathrm{~N}_{3}\right)$

\begin{tabular}{cccc}
\hline Entry & $\mathbf{R L i}$ (equiv) & $\mathbf{A r S O}_{2} \mathbf{N}_{3}$ & Yield $^{[\mathbf{a}]}$ \\
$\mathbf{1}$ & $t$-BuLi (2.1 equiv) & $2,4,6-\left(i-\mathrm{Pr}_{3} \mathrm{C}_{6} \mathrm{H}_{2} \mathrm{SO}_{2} \mathrm{~N}_{3}\right.$ & $76 \%$ \\
$\mathbf{2}$ & $t$-BuLi (2.1 equiv) & $4-\mathrm{Cl}_{-} \mathrm{C}_{6} \mathrm{H}_{4} \mathrm{SO}_{2} \mathrm{~N}_{3}$ & $63 \%$ \\
$\mathbf{3}$ & $t$-BuLi (2.1 equiv) & $\mathrm{TsN}_{3}$ & $77 \%$ \\
$\mathbf{4}$ & $n$-BuLi (1.1 equiv) & $\mathrm{TsN}_{3}$ & $95 \%$ \\
$\mathbf{5}^{[\mathbf{b}]}$ & $n$-BuLi (1.1 equiv) & $\mathrm{TsN}_{3}$ & $82 \%$ \\
\hline
\end{tabular}

[a] Isolated yield. [b] Iodine-lithium exchange was performed for $10 \mathrm{~min}$ at $0{ }^{\circ} \mathrm{C}$ (ice bath), $\mathrm{TsN}_{3}$ was added dropwise at $0{ }^{\circ} \mathrm{C}$ and the reaction mixture was warmed to rt over $16 \mathrm{~h}$.

In a dry Schlenk tube, iodoferrocene $(100 \mathrm{mg}, 0.321 \mathrm{mmol}, 1.0$ equiv) was placed and the Schlenk tube was evacuated and backfilled with argon $(3 \times)$. Anhydrous THF $(2 \mathrm{~mL})$ was added and the resulting solution was cooled to $-78{ }^{\circ} \mathrm{C}$ (acetone/dry ice). $\mathbf{R L i}$ was added dropwise and the mixture was stirred for $30 \mathrm{~min}$. Then, $\mathbf{A r S O}_{2} \mathbf{N}_{3}$ (1.2 equiv; $1.0 \mathrm{M}$ in THF) was added dropwise at $-78{ }^{\circ} \mathrm{C}$ and the resulting dark red solution was stirred at this temperature for $10 \mathrm{~min}$. The cooling bath was removed, the Schlenk tube was wrapped in aluminum foil and the mixture was stirred for $16 \mathrm{~h}$ at $\mathrm{rt}$. After exposure to air, $\mathrm{NaHCO}_{3}$ (sat. aq., $20 \mathrm{~mL}$ ) and EtOAc $(20 \mathrm{~mL})$ were added. The aqueous layer was separated and extracted with EtOAc $(2 \times 20 \mathrm{~mL})$. The combined organic layers were dried $\left(\mathrm{Na}_{2} \mathrm{SO}_{4}\right)$, filtered, and concentrated under reduced pressure. Azidoferrocene (2a) was isolated by gravity column chromatography (basic $\mathrm{Al}_{2} \mathrm{O}_{3}$, activity grade $\mathrm{I}+5 \mathrm{wt} \% \mathrm{H}_{2} \mathrm{O}$ ). 


\section{Initial attempts to accelerate the fragmentation of the triazene intermediate in batch.}

As a preliminary investigation for the flow protocol, it was attempted to accelerate the fragmentation of the triazene species in batch at elevated temperature.

In a dry Schlenk tube, 1a (100 mg, $0.321 \mathrm{mmol}, 1.0$ equiv) was placed and the Schlenk tube was evacuated and backfilled with argon $(3 \times)$. Anhydrous THF $(2 \mathrm{~mL})$ was added and the resulting solution was cooled to $-78{ }^{\circ} \mathrm{C}$ (acetone/dry ice). $n$-Butyllithium $(0.141 \mathrm{~mL}, 0.353 \mathrm{mmol}, 1.1$ equiv; $2.5 \mathrm{M}$ in $n$ hexane) was added dropwise and the mixture was stirred for $30 \mathrm{~min}$. Tosyl azide $(0.513 \mathrm{~mL}$, $0.383 \mathrm{mmol}, 1.2$ equiv; $1.0 \mathrm{M}$ in THF) was added dropwise at $-78{ }^{\circ} \mathrm{C}$. Then, the Schlenk tube was removed from the cooling bath and immersed in a preheated oil bath at the temperature stated. The solution was stirred at this temperature for the time indicated. The reaction progress was monitored by TLC. After exposure to air, $\mathrm{NaHCO}_{3}$ (sat. aq., $20 \mathrm{~mL}$ ) and EtOAc $(20 \mathrm{~mL})$ were added. The aqueous layer was separated and extracted with EtOAc $(2 \times 20 \mathrm{~mL})$. The combined organic layers were dried $\left(\mathrm{Na}_{2} \mathrm{SO}_{4}\right)$, filtered, and concentrated under reduced pressure. Azidoferrocene (2a) was isolated by gravity column chromatography (basic $\mathrm{Al}_{2} \mathrm{O}_{3}$, activity grade $\mathrm{I}+5$ wt $\% \mathrm{H}_{2} \mathrm{O}, n$-pentane).

Table S2. Fragmentation of the triazene species at elevated temperature in batch

\begin{tabular}{cccc}
\hline Entry & Temperature $\left({ }^{\circ} \mathbf{C}\right)$ & Time (min) & Yield $^{[\mathrm{a}]}$ \\
$\mathbf{1}^{[\mathrm{b}]}$ & 50 & 10 & $62 \%$ \\
$\mathbf{2}^{[\mathrm{b}]}$ & 60 & 20 & $78 \%$ \\
$\mathbf{3}$ & 60 & 30 & $70 \%$ \\
\hline
\end{tabular}

[a] Isolated yield. [b] Incomplete fragmentation of the triazene species.

These observations indicate that complete fragmentation of the triazene species requires approximately $30 \mathrm{~min}$ at $60{ }^{\circ} \mathrm{C}$ (entry 3). However, azidoferrocene (2a) can be isolated in a slightly higher yield although fragmentation was not complete when heating was performed only for $20 \mathrm{~min}$ (entry 2). This finding suggests that at $60{ }^{\circ} \mathrm{C}$ product 2a starts to decompose resulting in lower yields. 


\subsection{Optimization of Reaction Conditions for the Synthesis of Ferrocenyl}

Azides in Flow

\section{Attempts to perform iodine-lithium exchange and trapping with tosyl azide in a semi-batch procedure.}

To find suitable reaction parameters for the iodine-lithium exchange and subsequent trapping with tosyl azide in flow, semi-batch experiments were conducted. The iodine-lithium exchange and the subsequent trapping with tosyl azide were performed in flow, while fragmentation of the triazene species was completed in batch.

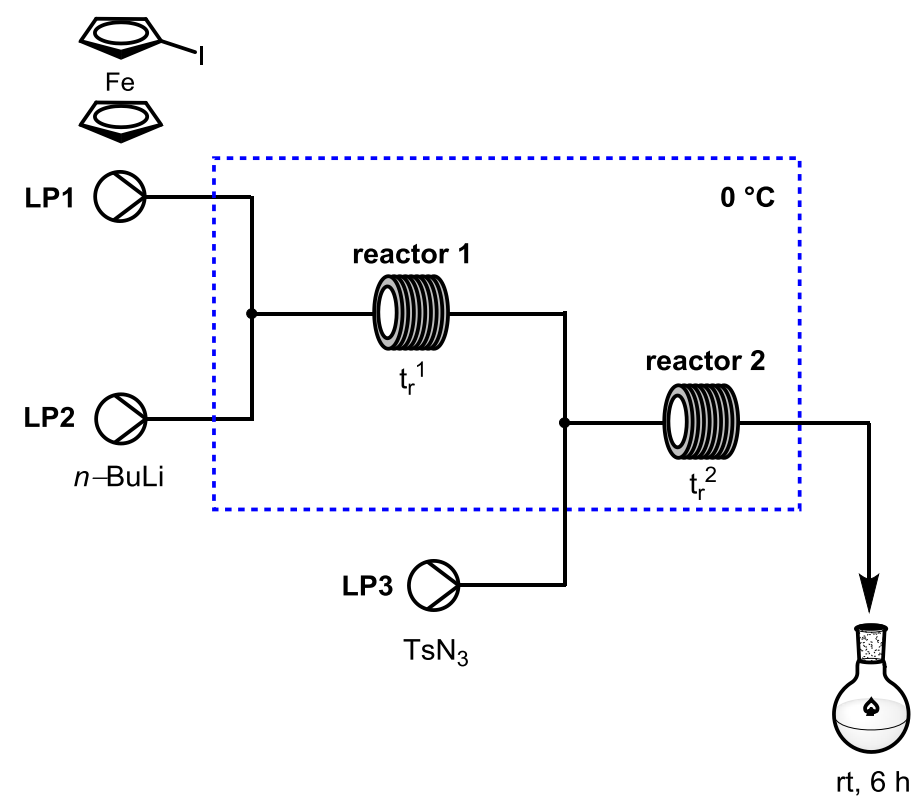

Syringe pumps (LP1-LP3) equipped with gas-tight $10 \mathrm{~mL}$ glass syringes were used to pump solutions of iodoferrocene (1a, $0.2 \mathrm{M}$ in THF, $\left.\mathrm{v}_{\mathrm{FcI}}\right), n$-butyllithium (1.2 $\mathrm{M} n$-hexane, $\mathrm{v}_{n \text {-BuLi }}$ ), and tosyl azide $\left(0.75 \mathrm{M}\right.$ in THF, $\left.\mathrm{v}_{\mathrm{TsN} 3}\right)$. All solutions were pumped through precooling loops (FEP tube, outer diameter $1 / 16$ ", inner diameter $1 / 32 ", V=0.15 \mathrm{~mL}$ ) before mixing. The solutions of iodoferrocene and $n$ butyllithium were merged in a T-mixer or a static mixer and pumped through tube reactor 1 with $t_{R}{ }^{1}$. At the end of this reactor, the reaction stream was merged with a stream of tosyl azide in a T-mixer and pumped through tube reactor 2 (FEP tube, outer diameter 1/16", inner diameter $1 / 32$ ", $V=1 \mathrm{~mL}$ ) with $t_{R}{ }^{2}$. The outlet of this tube reactor was connected to a flask or a dry Schlenk tube. Reactor 1, reactor 2 , the mixing units and the precooling loops were cooled to $0{ }^{\circ} \mathrm{C}$ by immersion to an ice bath.

The syringe pumps were operated at the given flow rates. After an equilibration time of 3 residence times, a sample of $0.2 \mathrm{mmol}$ was collected in a dry Schlenk tube. The collected reaction mixture was stirred at $\mathrm{rt}$ for $6 \mathrm{~h}$. After exposure to air, $\mathrm{NaHCO}_{3}$ (sat. aq., $20 \mathrm{~mL}$ ) and EtOAc $(20 \mathrm{~mL})$ were added. The aqueous layer was separated and extracted with EtOAc $(2 \times 20 \mathrm{~mL})$. The combined organic layers were dried $\left(\mathrm{Na}_{2} \mathrm{SO}_{4}\right)$, filtered and concentrated under reduced pressure. Azidoferrocene (2a) was isolated by gravity column chromatography (basic $\mathrm{Al}_{2} \mathrm{O}_{3}$, activity grade $\mathrm{I}+5 \mathrm{wt} \% \mathrm{H}_{2} \mathrm{O}, n$-pentane). 
Table S3. Screening of parameters for iodine-lithium exchange and subsequent trapping with tosyl azide in a semi-batch procedure

\begin{tabular}{ccccccc}
\hline Entry & $\mathbf{v}_{\text {FcI }}[\mathbf{m L} / \mathbf{m i n}]$ & $\mathbf{v}_{\boldsymbol{n} \text {-BuLi }}[\mathbf{m L} / \mathbf{m i n}]$ & $\mathbf{v}_{\text {TsN3 }}[\mathbf{m L} / \mathbf{m i n}]$ & $\mathbf{t}_{\mathbf{R}}{ }^{\mathbf{1}}[\mathbf{s}]$ & $\mathbf{t}_{\mathbf{R}} \mathbf{2}[\mathbf{s}]$ & $\mathbf{Y i e l d}^{[\mathbf{e}]}$ \\
$\mathbf{1}^{[\mathbf{a}]}$ & 1.0 & 0.2 & 0.4 & 8 & 38 & $43 \%$ \\
$\mathbf{2}^{[\mathbf{b}]}$ & 1.0 & 0.2 & 0.4 & 17 & 38 & $67 \%$ \\
$\mathbf{3}^{[\mathbf{b}]}$ & 2.0 & 0.4 & 0.8 & 9 & 19 & $71 \%$ \\
$\mathbf{4}^{[\mathbf{c}]}$ & 2.0 & 0.4 & 0.8 & 0.5 & 19 & $82 \%$ \\
$\mathbf{5}^{[\mathbf{c}, \mathbf{d}]}$ & 2.0 & 0.4 & 0.8 & 0.5 & 19 & $87 \%$ \\
\hline
\end{tabular}

[a] Reactor 1: FEP tube (inner diameter 1/32", $\mathrm{V}=0.15 \mathrm{~mL}$ ) was used. [b] Reactor 1: FEP tube (inner diameter 1/32", V = $0.34 \mathrm{~mL}$ ) was used. [c] Reactor 1: stainless steel capillary (inner diameter $250 \mu \mathrm{m}$, $\mathrm{V}=20 \mu \mathrm{L}$ ) was used. [d] A static mixer with an internal volume of $20 \mu \mathrm{L}$ was used for the iodine-lithium exchange. [e] Isolated yield.

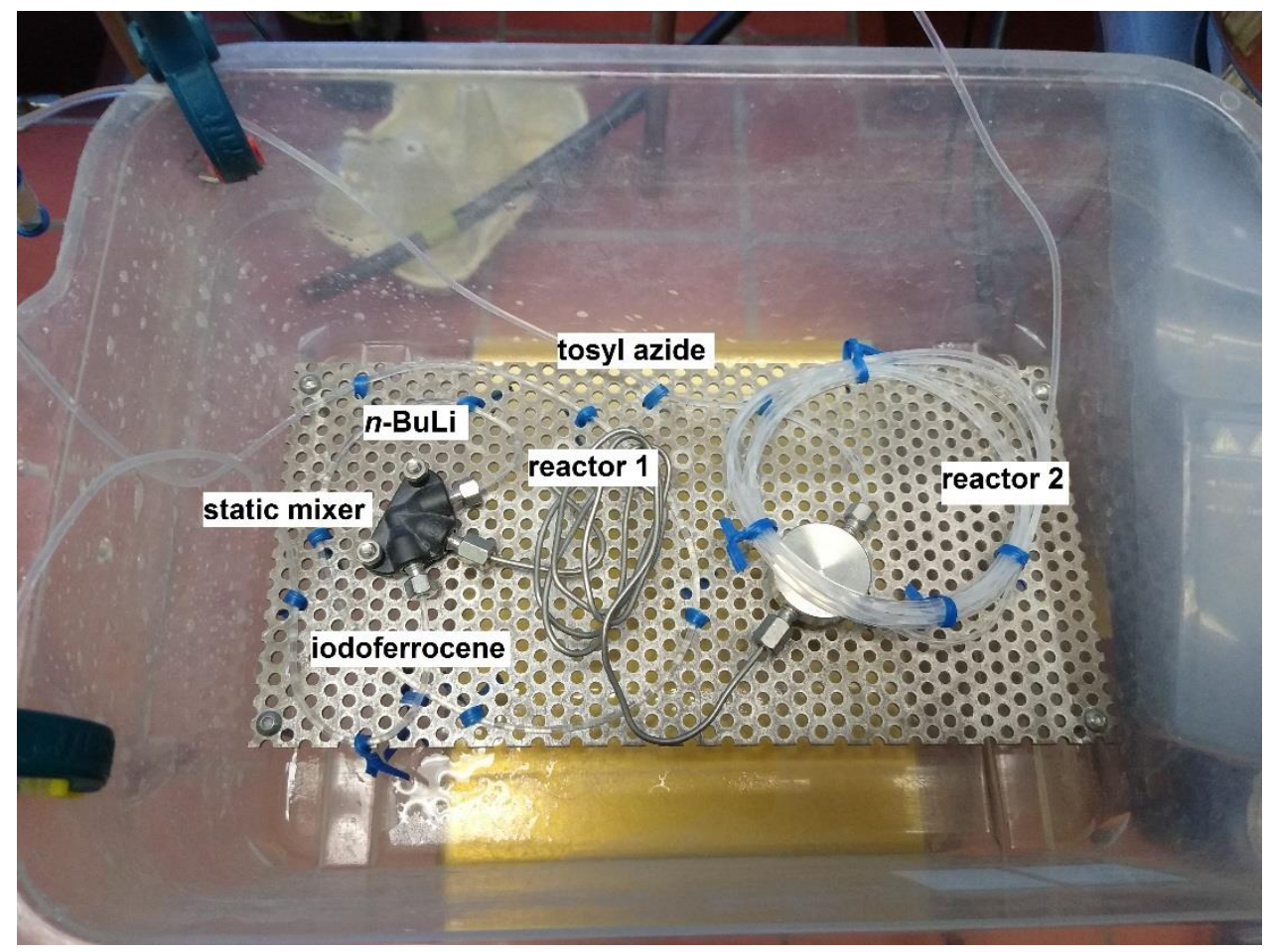

Picture of the self-assembled reactor plate: Iodoferrocene and $n$-butyllithium are merged in the static mixer and then pumped through reactor 1 . The reaction stream is merged with tosyl azide and then reacted in reactor 2 . 


\section{Attempts to accelerate the fragmentation of the triazene intermediate in a semi-batch procedure.}

In order to accelerate the fragmentation of the triazene intermediate in flow, semi-batch experiments were conducted. To prevent blocking of the tubings from precipitating salts, the reaction mixture was pumped as a segmented flow through a heated tube reactor resulting in a triphasic flow regime. ${ }^{[4]}$

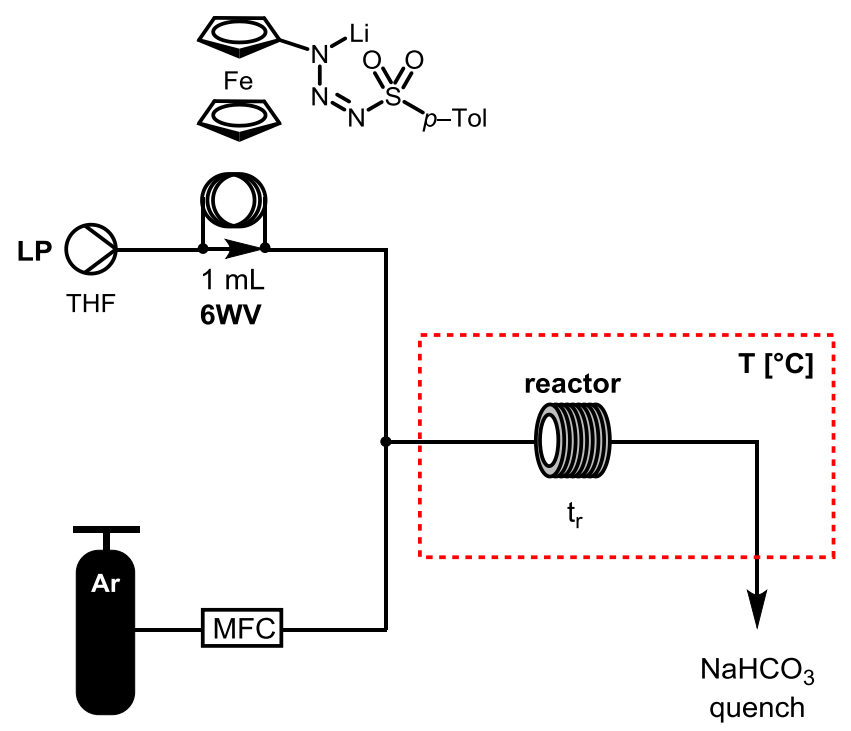

A HPLC pump (LP) to feed the solvent was connected to a HPLC injection valve (6WV) equipped with a $1 \mathrm{~mL}$ sample loop. The liquid supply was merged in a T-mixer with a stream of argon supplied by a mass flow controller (MFC). The resulting segmented flow was pumped through the tube reactor. The tube reactor with the volume $\mathbf{V}$ was immersed to a water bath at the given temperature. The reaction mixture was collected in a flask containing $\mathrm{NaHCO}_{3}$ (sat. aq., $20 \mathrm{~mL}$ ).

The pump and the reactor were washed with anhydrous THF for 2 residence times by pumping THF with the HPLC pump and simultaneously feeding argon using the MFC.

In a dry Schlenk tube, iodoferrocene $\mathbf{1 a}(100 \mathrm{mg}, 0.321 \mathrm{mmol}, 1.0$ equiv) was placed and the Schlenk tube was evacuated and backfilled with argon $(3 \times)$. Anhydrous THF $(1.1 \mathrm{~mL})$ was added and the resulting solution was cooled to $-78{ }^{\circ} \mathrm{C}$ (acetone/dry ice). $n$-Butyllithium (0.147 mL, $0.353 \mathrm{mmol}, 1.1$ equiv) was added dropwise and the mixture was stirred for $30 \mathrm{~min}$. Then, tosyl azide (1.2 equiv; $1.0 \mathrm{M}$ in THF) was added dropwise at $-78^{\circ} \mathrm{C}$. Next, the solution was loaded on the sample loop of the HPLC injection valve. The solution was immediately injected by switching from the load to the inject position and the solution was pumped through the reactor at the given flow rate and temperature. The collected reaction mixture was diluted with EtOAc $(20 \mathrm{~mL})$. The aqueous layer was separated and extracted with EtOAc $(2 \times 20 \mathrm{~mL})$. The combined organic layers were dried $\left(\mathrm{Na}_{2} \mathrm{SO}_{4}\right)$, filtered and concentrated under reduced pressure. Azidoferrocene (2a) was isolated by gravity column chromatography (basic $\mathrm{Al}_{2} \mathrm{O}_{3}$, activity grade $\mathrm{I}+5 \mathrm{wt} \% \mathrm{H}_{2} \mathrm{O}, n$-pentane). 
Table S4. Fragmentation of the triazene species at elevated temperature in flow

\begin{tabular}{rcccccc}
\hline Entry & $\mathbf{V}_{\text {liquid }}[\mathbf{m L} / \mathbf{m i n}]$ & $\mathbf{V}_{\text {argon }}[\mathbf{m L} / \mathbf{m i n}]$ & $\mathbf{V}[\mathbf{m L}]$ & $\mathbf{T}\left[{ }^{\circ} \mathbf{C}\right]$ & $\mathbf{t}_{\mathbf{R}}{ }^{[a]}[\mathbf{m i n}]$ & $\mathbf{Y i e l d}^{[\mathbf{d}]}$ \\
$\mathbf{1}^{[\mathbf{a}, \mathbf{c}]}$ & 0.4 & 0.4 & 10 & 50 & 10 & $49 \%$ \\
$\mathbf{2}^{[\mathbf{a}, \mathbf{c}]}$ & 0.4 & 0.4 & 10 & 55 & 13 & $74 \%$ \\
$\mathbf{3}^{[\mathbf{a}, \mathbf{c}]}$ & 0.2 & 0.2 & 10 & 60 & 15 & $85 \%$ \\
$\mathbf{4}^{[\mathbf{b}]}$ & 1.3 & 1.0 & 40 & 60 & 6 & n.d. \\
$\mathbf{5}^{[\mathbf{b}]}$ & 3.0 & 0.3 & 40 & 60 & 15 & $85 \%$ \\
\hline
\end{tabular}

[a] A FEP tube reactor (inner diameter of 1/32") was used. [b] A PTFE tube reactor (inner diameter 1/16") was used. [c] Temporary blockage of the tube reactor was observed. [d] Isolated yield. N.d. = not determined.

Due to expansion of the argon slugs and partial evaporation of the THF, the observed residence times are significantly lower than calculated.

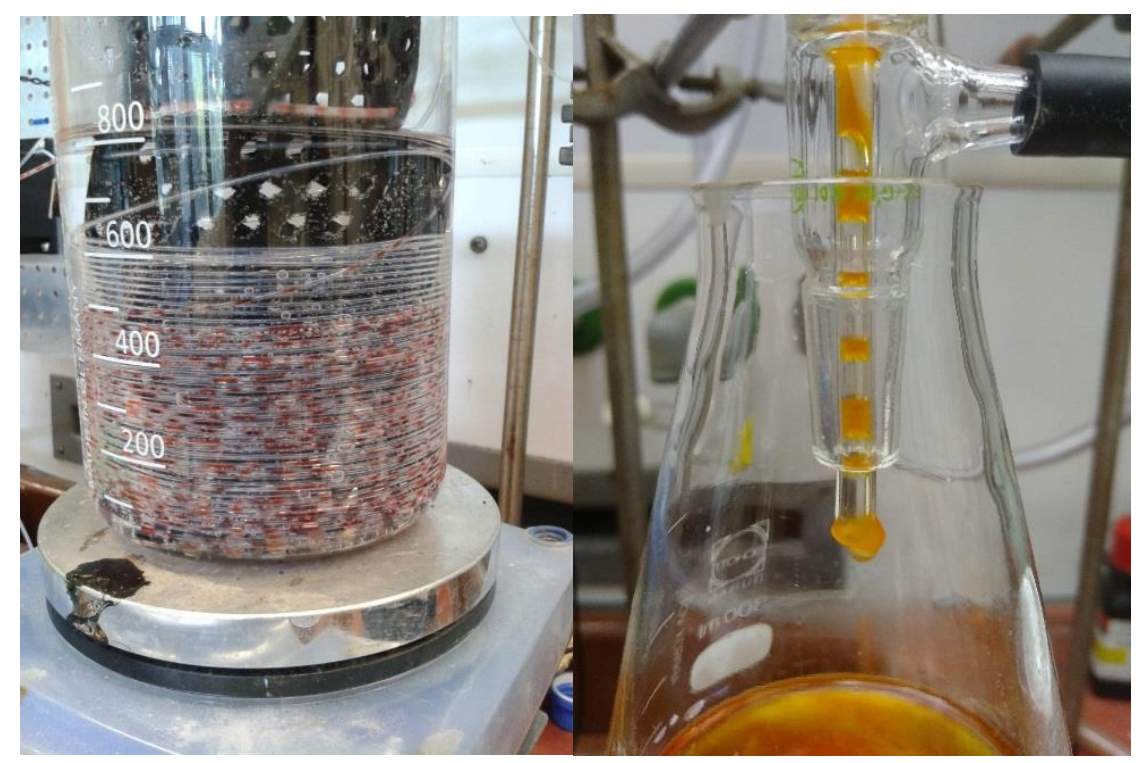

Left: Picture of the tube reactor when the reaction mixture (red) is pumped through the reactor in a segmented flow; Right: Picture of the triphasic flow regime on the outlet of the reactor. 


\section{Optimization of parameters of the combined flow setup}

Finally, the complete setup was constructed by combining both semi-batch procedures.

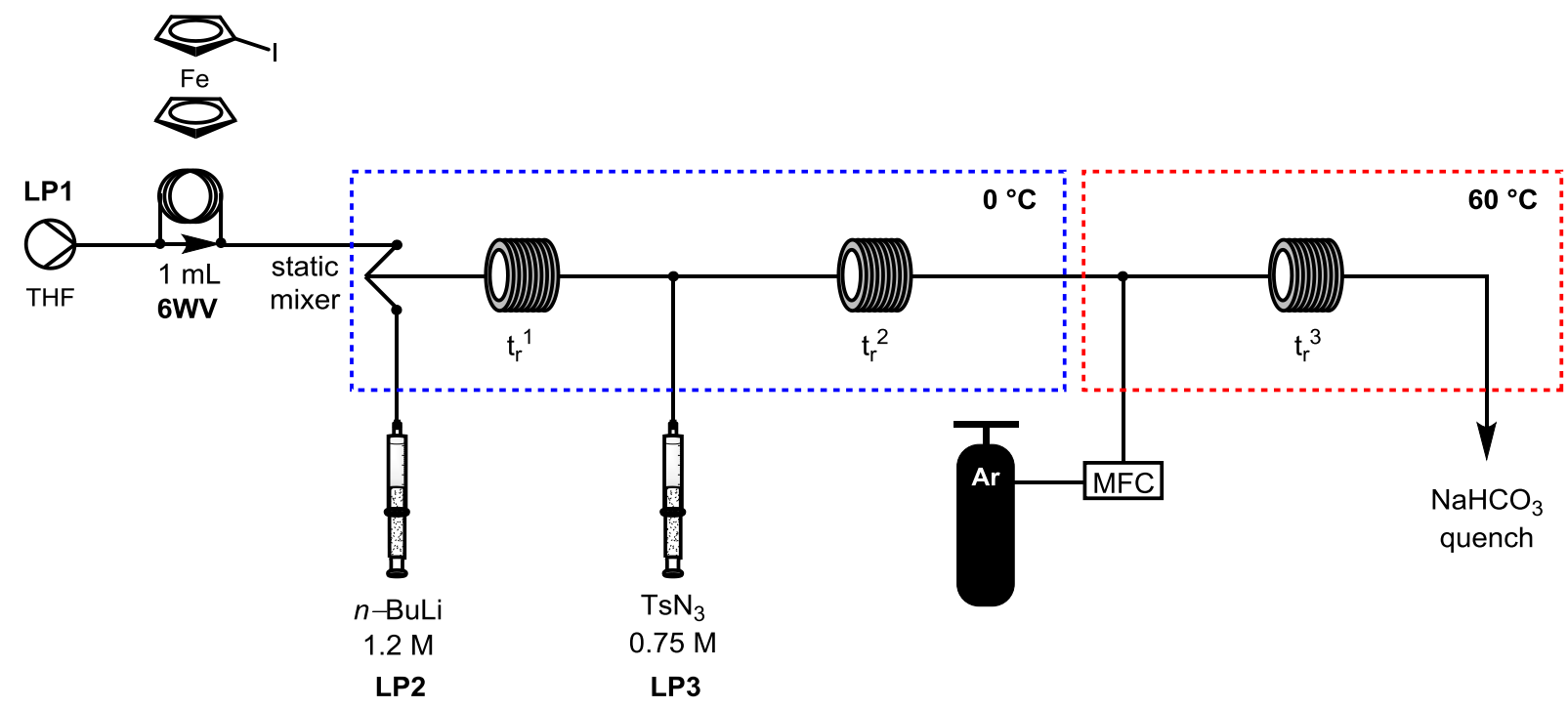

Syringe pumps (LP2, LP3) equipped with gastight $10 \mathrm{~mL}$ glass-syringes were used to pump solutions of $n$-butyllithium ( $1.2 \mathrm{M}$ in $n$-hexane, $\left.\mathrm{v}_{n \text {-BuLi }}\right)$ and tosyl azide $\left(0.75 \mathrm{M}\right.$ in THF, $\left.\mathrm{v}_{\mathrm{TsN}}\right)$. Both solutions were passed through precooling loops (FEP tube, outer diameter 1/16", inner diameter $1 / 32$ ", $\mathrm{V}=0.15 \mathrm{~mL}$ ) before mixing.

A HPLC pump (LP1) to feed the solvent was connected to a HPLC injection valve (6WV) equipped with a $1 \mathrm{~mL}$ sample loop. The liquid supply was passed through a precooled loop (FEP tube, outer diameter $1 / 16$ ", inner diameter $1 / 32 ", V=0.15 \mathrm{~mL}$ ) and then merged in a static mixer (internal volume: $20 \mu \mathrm{L})$ with a solution of $n$-butyllithium (1.2 M in $n$-hexane, $\left.\mathrm{v}_{n-\text {-BuLi }}\right)$. The resulting reaction stream was pumped through tube reactor1 (stainless steel capillary, inner diameter $250 \mu \mathrm{m}, \mathrm{V}=20 \mu \mathrm{L}$ ) and then merged in a stainless steel T-mixer with a solution of tosyl azide. Reactor1, reactor2, the mixing units and the precooled loops were cooled to $0{ }^{\circ} \mathrm{C}$ by immersion to an ice bath.

The reaction stream was mixed in a T-mixer with argon provided by a mass flow controller (MFC) resulting in a segmented flow pattern. This stream was pumped through tube reactor3 (PTFE tube, inner diameter $1 / 8 ", V=40 \mathrm{~mL}$ ) heated to $60^{\circ} \mathrm{C}$ in a water bath. The resulting triphasic flow regime was collected in a flask containing $\mathrm{NaHCO}_{3}$ (sat. aq., $50 \mathrm{~mL}$ ).

The HPLC pump and the reactor were washed with anhydrous THF for 2 residence times by pumping THF with the HPLC pump and feeding argon using the MFC. A solution of iodoferrocene (1a) was loaded on the HPLC injection valve. The solutions of $n$-butyllithium and tosyl azide were pumped at the given flow rate for 15-20 s. Then, the solution of iodoferrocene (1a) was injected into the system by switching the HPLC valve to the "inject" position. The collected reaction mixture was diluted with EtOAc $(50 \mathrm{~mL})$. The aqueous layer was separated and extracted with EtOAc $(2 \times 50 \mathrm{~mL})$. The combined organic layers were dried $\left(\mathrm{Na}_{2} \mathrm{SO}_{4}\right)$, filtered, and concentrated under reduced pressure. Azidoferrocene 
(2a) was isolated by gravity column chromatography (basic $\mathrm{Al}_{2} \mathrm{O}_{3}$, activity grade $\mathrm{I}+5 \mathrm{wt} \% \mathrm{H}_{2} \mathrm{O}$, $n$-pentane).

Table S5. Screening of parameters for the combined flow setup

\begin{tabular}{ccccccccc}
\hline Entry & $\begin{array}{c}\mathbf{v}_{\text {FcI }} \\
{[\mathbf{m L} / \mathbf{m i n}]}\end{array}$ & $\begin{array}{c}\mathbf{v}_{\boldsymbol{n} \text {-BuLi }} \\
{[\mathbf{m L} / \mathbf{m i n}]}\end{array}$ & $\begin{array}{c}\mathbf{v}_{\text {TsN3 }} \\
{[\mathbf{m L} / \mathbf{m i n}]}\end{array}$ & $\begin{array}{c}\mathbf{V}_{\text {argon }} \\
{[\mathbf{m L} / \mathbf{m i n}]}\end{array}$ & $\begin{array}{c}\mathbf{t}_{\mathbf{R}}{ }^{1} \\
{[\mathbf{s}]}\end{array}$ & $\begin{array}{c}\mathbf{t}_{\mathbf{R}}{ }^{2} \\
{[\mathbf{s}]}\end{array}$ & $\begin{array}{c}\mathbf{t}_{\mathbf{R}}{ }^{3} \\
{[\mathbf{m i n}]}\end{array}$ & $\mathbf{Y i e l d}^{[\mathbf{c}]}$ \\
\hline $\mathbf{1}^{[\mathbf{a}]}$ & 2.0 & 0.2 & 0.4 & 0.5 & 0.55 & 24 & 12 & 71 \\
$\mathbf{2}^{[\mathbf{a}]}$ & 2.0 & 0.2 & 0.4 & 0.4 & 0.55 & 24 & 14 & 77 \\
$\mathbf{3}^{[\mathbf{b}]}$ & 1.0 & 0.2 & 0.4 & 0.5 & 1 & 38 & 18 & 62 \\
$\mathbf{3}^{[\mathbf{b}]}$ & 2.0 & 0.4 & 0.8 & 0.4 & 0.5 & 19 & 8 & 51 \\
$\mathbf{4}^{[\mathbf{b}]}$ & 2.0 & 0.4 & 0.8 & 0.2 & 0.5 & 19 & 15 & 82 \\
\hline
\end{tabular}

[a] A $0.1 \mathrm{M}$ solution of iodoferrocene was employed. [b] A $0.2 \mathrm{M}$ solution of iodoferrocene was employed. [c] Isolated yield. 


\subsection{Synthesis of Substrates}

Synthesis of tosyl azide (S1)

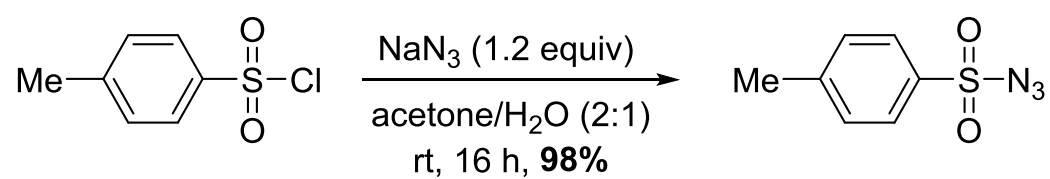

In a $250 \mathrm{~mL}$ round bottom flask, tosyl chloride (10.0 g, $52.5 \mathrm{mmol}, 1.0$ equiv) was dissolved in acetone $(80 \mathrm{~mL})$. Under ice bath cooling, a solution of sodium azide ( $3.75 \mathrm{~g}, 57.7 \mathrm{mmol}, 1.1$ equiv) in $\mathrm{H}_{2} \mathrm{O}(40 \mathrm{~mL})$ was added over $10 \mathrm{~min}$ using a dropping funnel. The resulting suspension was stirred at $\mathrm{rt}$ for $1 \mathrm{~h}$. Then, the suspension was diluted with $\mathrm{NaCl}$ (sat. aq., $50 \mathrm{~mL}$ ) and $\mathrm{EtOAc}(50 \mathrm{~mL})$. The aqueous layer was separated and extracted with EtOAc $(2 \times 100 \mathrm{~mL})$. The combined organic layers were dried $\left(\mathrm{Na}_{2} \mathrm{SO}_{4}\right)$, filtered, and concentrated under reduced pressure. The title compound $\mathbf{S 1}$ (5.09 g, $25.8 \mathrm{mmol}$, $98 \%$ ) was obtained without further purification as a colorless oil that solidified at $8{ }^{\circ} \mathrm{C}$.

$\mathrm{Rf}=0.49$ (n-hexane/EtOAc 9:1).

${ }^{1} \mathrm{H} \mathrm{NMR}\left(500 \mathrm{MHz}, \mathrm{CDCl}_{3}\right) \delta[\mathrm{ppm}]=7.85(\mathrm{~d}, J=8.2 \mathrm{~Hz}, 2 \mathrm{H}), 7.41(\mathrm{~d}, J=8.2 \mathrm{~Hz}, 2 \mathrm{H}), 2.48(\mathrm{~s}, 3 \mathrm{H})$.

${ }^{13} \mathrm{C}\left\{{ }^{1} \mathrm{H}\right\} \mathrm{NMR}\left(126 \mathrm{MHz}, \mathrm{CDCl}_{3}\right) \delta[\mathrm{ppm}]=146.3,135.7,130.4,127.7,21.9$.

The spectroscopic data are consistent with those reported in the literature. ${ }^{[2]}$

\section{Preparation of a stock solution of tosyl azide in THF:}

In a dry Schlenk tube, $\mathrm{CaH}_{2}(\sim 100 \mathrm{mg}$ for $1 \mathrm{~g}$ of tosyl azide) was placed and the tube was evacuated and backfilled with argon (3x). A defined mass of tosyl azide was added as oil by using a Pasteur pipette. Then, a defined volume of anhydrous THF was added to give a concentration of 0.5-2.5 M. The resulting solution of tosyl azide in THF was dried over $\mathrm{CaH}_{2}$ at least for $16 \mathrm{~h}$ prior use (gas evolution!). The solution was stored over $\mathrm{CaH}_{2}$ at $8{ }^{\circ} \mathrm{C}$ in the dark and could be used for several months.

\section{Synthesis of iodoferrocene (1a)}

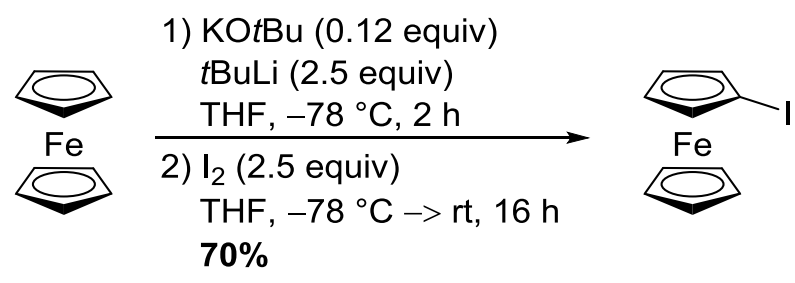

This compound was prepared according to a reported procedure with slight modifications. ${ }^{[5]}$

In a dry $100 \mathrm{~mL}$ Schlenk tube, ferrocene $(3.00 \mathrm{~g}, 16.1 \mathrm{mmol}, 1.0$ equiv) and potassium tert-butoxide (217 mg, $1.94 \mathrm{mmol}, 0.12$ equiv) were placed and the flask was evacuated and backfilled with argon $(3 \times)$. Then, anhydrous THF $(45 \mathrm{~mL})$ was added and the resulting yellow solution was cooled to - 
$78{ }^{\circ} \mathrm{C}$ (acetone/dry ice). tert-Butyllithium ( $23.7 \mathrm{~mL}, 40.3 \mathrm{mmol}, 2.5$ equiv., $1.7 \mathrm{M}$ in $n$-pentane) was added dropwise and the resulting orange mixture was stirred at $-78^{\circ} \mathrm{C}$ for $2 \mathrm{~h}$. Then, solid iodine $(10.2 \mathrm{~g}$, $40.3 \mathrm{mmol}, 2.5$ equiv) was added portionwise over $10 \mathrm{~min}$ and the mixture was warmed to rt over $16 \mathrm{~h}$ by removing the cold bath. Then, the dark brown mixture was diluted with $\mathrm{Na}_{2} \mathrm{~S}_{2} \mathrm{O}_{3}$ (sat. aq., $100 \mathrm{~mL}$ ) and EtOAc $(50 \mathrm{~mL})$. The aqueous layer was separated and extracted with EtOAc $(3 \times 100 \mathrm{~mL})$. The combined organic layers were dried $\left(\mathrm{MgSO}_{4}\right)$, filtered, concentrated under reduced pressure, and loaded onto Celite ${ }^{\circledR}$. Flash column chromatography $\left(\mathrm{SiO}_{2}, n\right.$-pentane) followed by removement of remaining ferrocene by sublimation at $60{ }^{\circ} \mathrm{C} / 2 \mathrm{mbar}$ on a rotary evaporator gave the title compound $1 \mathrm{a}(3.52 \mathrm{~g}$, $11.3 \mathrm{mmol}, 70 \%)$ as a red oil that solidified upon standing.

If necessary, the product can be further purified by recrystallization from $n$-pentane at $-78^{\circ} \mathrm{C}$.

$R_{\mathrm{f}}=0.67\left(\mathrm{SiO}_{2} ; n\right.$-pentane; vis. vanillin $)$.

${ }^{1} \mathrm{H}$ NMR $\left(500 \mathrm{MHz}, \mathrm{CDCl}_{3}\right): \delta[\mathrm{ppm}]=4.41(\mathrm{t}, J=1.9 \mathrm{~Hz}, 2 \mathrm{H}), 4.19(\mathrm{~s}, 5 \mathrm{H}), 4.15(\mathrm{t}, J=1.9 \mathrm{~Hz}, 2 \mathrm{H})$.

${ }^{13} \mathrm{C}\left\{{ }^{1} \mathrm{H}\right\}$ NMR $\left(126 \mathrm{MHz}, \mathrm{CDCl}_{3}\right): \delta[\mathrm{ppm}]=74.6,71.2,69.0,39.8$.

The spectroscopic data are consistent with those reported in the literature. ${ }^{[5]}$

\section{Synthesis of 1,1'-dibromoferrocene (1b)}

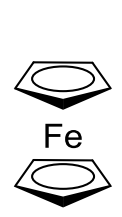

1) $n$ BuLi (2.4 equiv) TMEDA (2.4 equiv) $\frac{n \text {-hexane, } \mathrm{rt}, 16 \mathrm{~h}}{\text { 2) }\left(\mathrm{CBrCl}_{2}\right)_{2}(2.5 \text { equiv })}$ THF, $-78^{\circ} \mathrm{C} \rightarrow \mathrm{rt}, 16 \mathrm{~h}$ $81 \%$

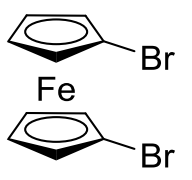

This compound was prepared according to a reported procedure with slight modifications. ${ }^{[5]}$

In a dry $250 \mathrm{~mL}$ Schlenk flask equipped with a large, conical stirring bar, ferrocene $(5.0 \mathrm{~g}, 27 \mathrm{mmol}$, 1.0 equiv) was placed and the flask was evacuated and backfilled with argon (3x). Then, anhydrous and degassed $n$-hexane $(50 \mathrm{~mL})$ and anhydrous tetramethylethylene diamine $(9.7 \mathrm{~mL}, 65 \mathrm{mmol}, 2.4$ equiv) were added via syringe. While stirring, $n$-butyllithium ( $26 \mathrm{~mL}, 65 \mathrm{mmol}, 2.4$ equiv; $2.5 \mathrm{M}$ in $n$-hexane) was added via syringe and the solution was stirred for $16 \mathrm{~h}$ at $\mathrm{rt}$ (gas evolution!). During this time, an orange precipitate was formed. The solution was removed via syringe and the precipitate was washed with anhydrous $n$-hexane $(1 \times 15 \mathrm{~mL})$. The precipitate was dissolved in anhydrous THF $(50 \mathrm{~mL})$ and cooled to $-78^{\circ} \mathrm{C}$ (acetone/dry ice). 1,2-Dibromotetrachloroethane ( $22 \mathrm{~g}, 67 \mathrm{mmol}, 2.5$ equiv) was added as solid in portions over $10 \mathrm{~min}$ in a continuous argon stream and the resulting brown mixture was warmed to rt over $16 \mathrm{~h} . \mathrm{NH}_{4} \mathrm{Cl}$ (sat. aq., $50 \mathrm{~mL}$ ) was added slowly and the resulting biphasic mixture was diluted with $\mathrm{H}_{2} \mathrm{O}(50 \mathrm{~mL})$. The organic layer was separated and the aqueous layer was extracted with $\mathrm{CH}_{2} \mathrm{Cl}_{2}(3 \times 100 \mathrm{~mL})$. The combined organic layers were dried $\left(\mathrm{MgSO}_{4}\right)$, filtered, concentrated under reduced pressure, and loaded onto $\mathrm{SiO}_{2}$. Flash column chromatography $\left(\mathrm{SiO}_{2} ; n\right.$-pentane $/ \mathrm{CH}_{2} \mathrm{Cl}_{2}$ 
100:0 to 100:5) followed by removal of remaining ferrocene by sublimation at $60{ }^{\circ} \mathrm{C}$ and $2 \mathrm{mbar}$ on a rotary evaporator gave the title compound $\mathbf{1 b}(7.5 \mathrm{~g}, 22 \mathrm{mmol}, 81 \%)$ as a red solid.

If necessary, the product can be further purified by recrystallization from $n$-pentane at $-78{ }^{\circ} \mathrm{C}$. $R_{\mathrm{f}}=0.52\left(\mathrm{SiO}_{2} ; n\right.$-hexane; vis. vanillin).

${ }^{1} \mathrm{H}$ NMR $\left(500 \mathrm{MHz}, \mathrm{CDCl}_{3}\right) \delta[\mathrm{ppm}]=4.42(\mathrm{t}, J=1.9 \mathrm{~Hz}, 4 \mathrm{H}), 4.17(\mathrm{t}, J=1.9 \mathrm{~Hz}, 4 \mathrm{H})$.

${ }^{13} \mathrm{C}\left\{{ }^{1} \mathrm{H}\right\} \mathrm{NMR}\left(126 \mathrm{MHz}, \mathrm{CDCl}_{3}\right) \delta[\mathrm{ppm}]=78.4,72.8,70.1$.

The spectroscopic data are consistent with those reported in the literature. ${ }^{[5]}$

\section{Synthesis of 1,1'-diiodoferrocene (1c):}

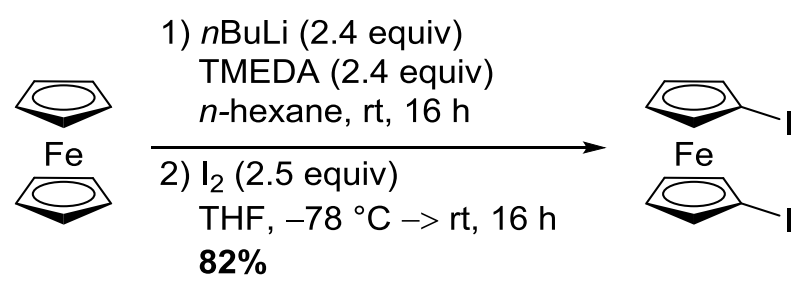

This compound was prepared according to a reported procedure with slight modifications. ${ }^{[5]}$

In a dry $250 \mathrm{~mL}$ Schlenk flask equipped with a large, conical stirring bar, ferrocene $(5.0 \mathrm{~g}, 27 \mathrm{mmol}$, 1 equiv) was placed and the flask was evacuated and backfilled with $\operatorname{argon}(3 \times)$. Then, anhydrous $n$ hexane $(50 \mathrm{~mL})$ and anhydrous tetramethylethylene diamine $(9.7 \mathrm{~mL}, 65 \mathrm{mmol}, 2.4$ equiv) were added via syringe. While stirring, $n$-butyllithium ( $26 \mathrm{~mL}, 65 \mathrm{mmol}, 2.4$ equiv; $2.5 \mathrm{M}$ in $n$-hexane) was added via syringe and the solution was stirred for $16 \mathrm{~h}$ at $\mathrm{rt}$ (gas evolution!). During this time, an orange precipitate was formed. The solution was removed via syringe and the residue was washed with anhydrous $n$-hexane $(1 \times 15 \mathrm{~mL})$. The precipitate was dissolved in anhydrous THF $(50 \mathrm{~mL})$ and cooled to $-78{ }^{\circ} \mathrm{C}$ (acetone/dry ice). Iodine ( $17 \mathrm{~g}, 67 \mathrm{mmol}, 2.5$ equiv) was added in portions over $10 \mathrm{~min}$ in a continuous argon stream and the resulting dark brown mixture was warmed to rt over night. The mixture was diluted with $\mathrm{Na}_{2} \mathrm{~S}_{2} \mathrm{O}_{3}$ (sat. aq., $100 \mathrm{~mL}$ ) and EtOAc (100 mL). The aqueous layer was separated and extracted with EtOAc $(3 \times 100 \mathrm{~mL})$. The combined organic layers were dried $\left(\mathrm{MgSO}_{4}\right)$, filtered, concentrated under reduced pressure, and loaded onto $\mathrm{SiO}_{2}$. Flash column chromatography $\left(\mathrm{SiO}_{2} ; n\right.$ pentane/ $/ \mathrm{CH}_{2} \mathrm{Cl}_{2}$ 100:0 to 100:5) followed by removal of remaining ferrocene by sublimation at $60{ }^{\circ} \mathrm{C} / 2 \mathrm{mbar}$ on a rotary evaporator gave the title compound $1 \mathrm{c}(9.7 \mathrm{~g}, 22 \mathrm{mmol}, 82 \%)$ as a brown oil. If necessary, the product can be further purified by recrystallization from $n$-pentane at $-78{ }^{\circ} \mathrm{C}$. $R_{\mathrm{f}}=0.48\left(\mathrm{SiO}_{2} ; n\right.$-hexane; vis. vanillin).

${ }^{1} \mathrm{H}$ NMR $\left(700 \mathrm{MHz}, \mathrm{CDCl}_{3}\right) \delta[\mathrm{ppm}]=4.37(\mathrm{t}, J=1.9 \mathrm{~Hz}, 4 \mathrm{H}), 4.18(\mathrm{t}, J=1.9 \mathrm{~Hz}, 4 \mathrm{H})$.

${ }^{13} \mathrm{C}\left\{{ }^{1} \mathrm{H}\right\} \mathrm{NMR}\left(176 \mathrm{MHz}, \mathrm{CDCl}_{3}\right) \delta[\mathrm{ppm}]=77.7,72.4,40.4$.

The spectroscopic data are consistent with those reported in the literature. ${ }^{[5]}$ 


\section{Synthesis of 1-bromo-1'-ferrocenylcarboxylic acid (S2)}

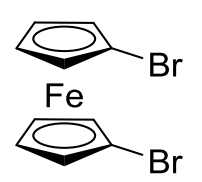

1) $n$ BuLi (1.0 equiv)

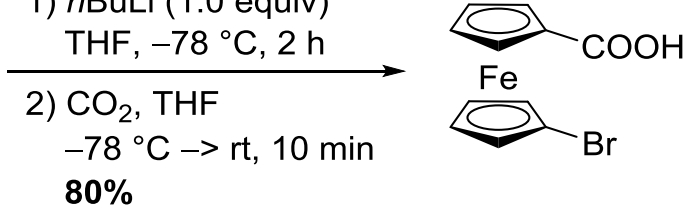

In a dry $100 \mathrm{~mL}$ Schlenk tube, $\mathbf{1 b}(1.0 \mathrm{~g}, 2.9 \mathrm{mmol}, 1$ equiv) was placed and the tube was evacuated and backfilled with argon $(3 \times)$. Anhydrous THF $(50 \mathrm{~mL})$ was added and the resulting orange solution was cooled to $-78{ }^{\circ} \mathrm{C}$ (acetone/dry ice). Then, $n$-butyllithium $(1.2 \mathrm{~mL}, 2.9 \mathrm{mmol}, 1.0$ equiv; $2.5 \mathrm{M}$ in $n$-hexane) was added dropwise at $-78^{\circ} \mathrm{C}$ and the resulting red solution was stirred at this temperature for $1 \mathrm{~h}$. At $-78{ }^{\circ} \mathrm{C}$, a stream of dry $\mathrm{CO}_{2}$ was bubbled through the solution for $10 \mathrm{~min}$ and the resulting yellow suspension was warmed to $\mathrm{rt}$ (gas evolution!). $\mathrm{NH}_{4} \mathrm{Cl}$ (sat. aq., $10 \mathrm{~mL}$ ) was added dropwise to the mixture. The solution was diluted with $\mathrm{NH}_{4} \mathrm{Cl}$ (sat. aq., $50 \mathrm{~mL}$ ) and EtOAc $(20 \mathrm{~mL}$ ). The aqueous layer was separated and extracted with EtOAc $(4 \times 50 \mathrm{~mL})$. The combined organic layers were dried $\left(\mathrm{MgSO}_{4}\right)$, filtered, and loaded onto Celite ${ }^{\circledR}$. Flash column chromatography $\left(\mathrm{SiO}_{2}, n\right.$-hexane/EtOAc 3:2 to $1: 1)$ gave the title compound $\mathbf{S 2}(0.72 \mathrm{~g}, 2.3 \mathrm{mmol}, 80 \%)$ as a red solid.

$R_{\mathrm{f}}=0.35\left(\mathrm{SiO}_{2} ; n\right.$-hexane/EtOAc 3:2; vis. vanillin) .

${ }^{1} \mathrm{H}$ NMR $\left(500 \mathrm{MHz}, \mathrm{CDCl}_{3}\right) \delta[\mathrm{ppm}]=4.91(\mathrm{t}, J=1.9 \mathrm{~Hz}, 2 \mathrm{H}), 4.51(\mathrm{t}, J=1.9 \mathrm{~Hz}, 2 \mathrm{H}), 4.49(\mathrm{t}$, $J=1.9 \mathrm{~Hz}, 2 \mathrm{H}), 4.20(\mathrm{t}, J=1.9 \mathrm{~Hz}, 2 \mathrm{H})$.

${ }^{13} \mathrm{C}\left\{{ }^{1} \mathrm{H}\right\}$ NMR $\left(176 \mathrm{MHz}, \mathrm{CDCl}_{3}\right) \delta[\mathrm{ppm}]=176.6,78.2,74.8,72.8,72.2,72.0,69.5$.

HRMS (ESI-TOF) $\mathrm{m} / \mathrm{z}$ calcd. for $\mathrm{C}_{11} \mathrm{H}_{10} \mathrm{BrFeO}_{2}{ }^{+}\left([\mathrm{M}+\mathrm{H}]^{+}\right)$308.9208; found: 308.9209 .

Note: A stream of $\mathrm{CO}_{2}$ gas was provided as follows: A $50 \mathrm{~mL}$ flask was charged with a few chunks of dry ice and sealed with a septum equipped with a Teflon tube providing a continuous stream of $\mathrm{CO}_{2}$ through the tube. A $25 \mathrm{~mL}$ flask was equipped with a septum and charged with conc. $\mathrm{H}_{2} \mathrm{SO}_{4}(10 \mathrm{~mL})$. The septum was equipped with a transfer canula not reaching in the conc. $\mathrm{H}_{2} \mathrm{SO}_{4}$. The Teflon tube of the flask containing the dry ice was introduced through the septum and immersed in the conc. $\mathrm{H}_{2} \mathrm{SO}_{4}$. In this way, a continuous stream of $\mathrm{CO}_{2}$-gas was bubbled through the conc. $\mathrm{H}_{2} \mathrm{SO}_{4}$ to dry the $\mathrm{CO}_{2}$. Through the transfer canula, a continuous stream of anhydrous $\mathrm{CO}_{2}$ is provided. After some minutes of flushing the system with $\mathrm{CO}_{2}$, the transfer canula was introduced into the reaction mixture. 


\section{Synthesis of 1-bromo-1'-ferrocenylcarboxylic acid tert-butyl ester (1e)}

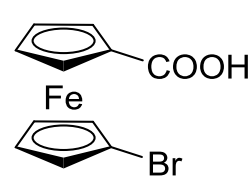
1) $(\mathrm{COCl})_{2}$ (3 equiv)
$\mathrm{CH}_{2} \mathrm{Cl}_{2}, \mathrm{rt}, 2 \mathrm{~h}$
2) $\mathrm{tBuOH}, 83^{\circ} \mathrm{C}, 2 \mathrm{~h}$
$86 \%$

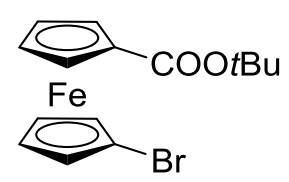

In a dry $25 \mathrm{~mL}$ Schlenk flask, S2 (600 mg, $1.94 \mathrm{mmol}, 1.0$ equiv) was placed and the flask was evacuated and backfilled with argon $(3 \times)$. Anhydrous $\mathrm{CH}_{2} \mathrm{Cl}_{2}(10 \mathrm{~mL})$ was added. To the resulting red suspension, oxalyl chloride ( $500 \mu \mathrm{L}, 5.83 \mathrm{mmol}, 3.0$ equiv) was added via syringe in one portion under water bath cooling. The resulting red solution was stirred at rt until no more gas evolution was observed (1-2 h). The red solution was concentrated under reduced pressure and under exclusion of moisture. Then, $t \mathrm{BuOH}(10 \mathrm{~mL})$ was added and the resulting red solution was heated to $83^{\circ} \mathrm{C}$. The mixture was diluted with $\mathrm{NaHCO}_{3}$ (sat. aq., $50 \mathrm{~mL}$ ) and EtOAc $(50 \mathrm{~mL})$. The aqueous layer was separated and extracted with EtOAc $(3 \times 50 \mathrm{~mL})$. The combined organic layers were dried $\left(\mathrm{MgSO}_{4}\right)$, filtered, and concentrated under reduced pressure, and loaded onto Celite ${ }^{\circledR}$. Flash column chromatography $\left(\mathrm{SiO}_{2}, n\right.$-hexane/EtOAc 10:1) gave the title compound $1 \mathrm{e}(607 \mathrm{mg}, 1.66 \mathrm{mmol}, 86 \%)$ as a red oil.

$R_{\mathrm{f}}=0.49\left(\mathrm{SiO}_{2} ; n\right.$-hexane/EtOAc 9:1; vis. vanillin $)$.

${ }^{1} \mathrm{H}$ NMR $\left(700 \mathrm{MHz}, \mathrm{CDCl}_{3}\right) \delta[\mathrm{ppm}]=4.75(\mathrm{t}, J=2.0 \mathrm{~Hz}, 2 \mathrm{H}), 4.41(\mathrm{t}, J=1.9 \mathrm{~Hz}, 2 \mathrm{H}), 4.39(\mathrm{t}$, $J=2.0 \mathrm{~Hz}, 2 \mathrm{H}), 4.14(\mathrm{t}, J=1.9 \mathrm{~Hz}, 2 \mathrm{H}), 1.57(\mathrm{~s}, 9 \mathrm{H})$.

${ }^{13} \mathrm{C}\left\{{ }^{1} \mathrm{H}\right\}$ NMR $\left(176 \mathrm{MHz}, \mathrm{CDCl}_{3}\right) \delta[\mathrm{ppm}]=170.0,80.6,78.1,75.2,73.7,72.7,71.8,68.9,28.5$.

HRMS (ESI-TOF) m/z calcd. for $\mathrm{C}_{15} \mathrm{H}_{17} \mathrm{BrFeO}_{2} \mathrm{Na}^{+}\left([\mathrm{M}+\mathrm{Na}]^{+}\right) 386.9653$; found: 386.9659 .

\section{Synthesis of 1-(1H-benzo[d][1,2,3]triazole)-1'-bromoferrocene (1f)}

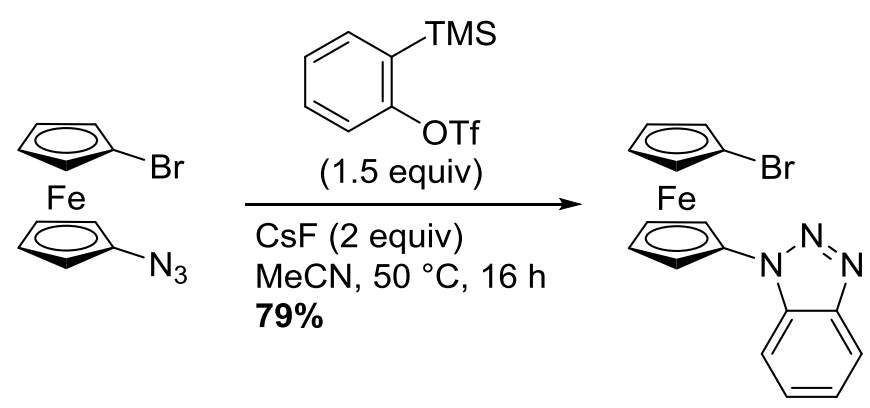

In a dry $20 \mathrm{~mL}$ Schlenk tube, finely powdered cesium fluoride (99.3 mg, $0.654 \mathrm{mmol}, 2.0$ equiv) was placed and the cesium fluoride was dried at $10^{-3} \mathrm{mbar}$ at $300{ }^{\circ} \mathrm{C}$ for $10 \mathrm{~min}$. After cooling to $\mathrm{rt}, 1$-azido1'-bromoferrocene $(\mathbf{2} \mathbf{b}, 100 \mathrm{mg}, 0.327 \mathrm{mmol}, 1.0$ equiv) was added and the tube was evacuated and backfilled with argon (3x). Then, anhydrous $\mathrm{MeCN}(4 \mathrm{~mL})$ was added and to the resulting orange solution, 2-(trimethylsilyl)phenyl trifluoromethanesulfonate $(0.120 \mathrm{~mL}, 0.491 \mathrm{mmol}, 1.5$ equiv) was added. The Schlenk tube was sealed and the mixture was stirred at $50{ }^{\circ} \mathrm{C}$ for $16 \mathrm{~h}$. After cooling to rt, the mixture was diluted with $\mathrm{NaHCO}_{3}$ (sat. aq., $50 \mathrm{~mL}$ ) and $\mathrm{CH}_{2} \mathrm{Cl}_{2}(50 \mathrm{~mL})$. The organic layer was separated and the aqueous layer was extracted with $\mathrm{CH}_{2} \mathrm{Cl}_{2}(3 \times 50 \mathrm{~mL})$. The combined organic layers 
were dried $\left(\mathrm{Na}_{2} \mathrm{SO}_{4}\right)$, filtered, and loaded onto Celite. Flash column chromatography $\left(\mathrm{SiO}_{2}\right.$, $n$-hexane/EtOAc 5:1) gave the title compound $1 \mathbf{f}(98.6 \mathrm{mg}, 0.258 \mathrm{mmol}, 79 \%)$ as an orange solid. $R_{\mathrm{f}}=0.37\left(\mathrm{SiO}_{2}, n\right.$-hexane/EtOAc 5:1).

${ }^{1} \mathrm{H}$ NMR $\left(400 \mathrm{MHz}, \mathrm{CDCl}_{3}\right) \delta[\mathrm{ppm}]=4.20(\mathrm{t}, J=1.9 \mathrm{~Hz}, 2 \mathrm{H}), 4.42(\mathrm{t}, J=2.0 \mathrm{~Hz}, 2 \mathrm{H}), 4.51(\mathrm{t}$, $J=1.9 \mathrm{~Hz}, 2 \mathrm{H}), 5.04(\mathrm{t}, J=2.0 \mathrm{~Hz}, 2 \mathrm{H}), 7.43(\mathrm{ddd}, J=8.4,7.0,1.0 \mathrm{~Hz}, 1 \mathrm{H}), 7.58(\mathrm{ddd}, J=8.4,7.0$, $1.0 \mathrm{~Hz}, 1 \mathrm{H}), 7.87(\mathrm{dt}, J=8.4,0.9 \mathrm{~Hz}, 1 \mathrm{H}), 8.12(\mathrm{dt}, J=8.4,0.9 \mathrm{~Hz}, 1 \mathrm{H})$.

${ }^{13} \mathrm{C}\left\{{ }^{1} \mathrm{H}\right\} \mathrm{NMR}\left(100 \mathrm{MHz}, \mathrm{CDCl}_{3}\right) \delta[\mathrm{ppm}]=146.6,133.0,128.0,124.4,120.5,110.9,95.2,78.5,72.0$, 69.6, 69.3, 64.5.

HRMS (ESI-TOF) m/z calcd. for $\mathrm{C}_{16} \mathrm{H}_{13} \mathrm{BrFeN}_{3}{ }^{+}\left([\mathrm{M}+\mathrm{H}]^{+}\right)$381.9637; found: 381.9648 .

\section{Synthesis of $(1 S, 4 S)$-1,7,7-trimethylbicyclo[2.2.1]hept-2-enyl trifluoromethanesulfonate (S3)}

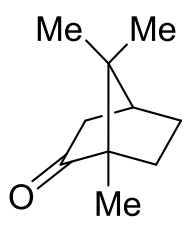

1) $(\mathrm{PPr})_{2} \mathrm{NLi}(1.2$ equiv)

$$
\text { THF, }-78^{\circ} \mathrm{C}, 2 \mathrm{~h}
$$

2) $\mathrm{PhNTf}_{2}(1.5$ equiv)

THF, $-78^{\circ} \mathrm{C} \rightarrow$ rt., $16 \mathrm{~h}$

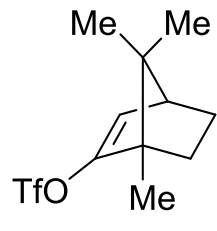

$72 \%$

This compound was prepared according to a reported procedure with slight modifications. ${ }^{[6]}$

In a dry $20 \mathrm{~mL}$ Schlenk tube, anhydrous diisopropylamine $(0.33 \mathrm{~mL}, 2.4 \mathrm{mmol}, 1.2$ equiv $)$ was placed and anhydrous THF $(2 \mathrm{~mL})$ was added. The solution was cooled to $-78{ }^{\circ} \mathrm{C}$ (acetone/dry ice). Then, $n$ butyllithium (1.1 mL, $2.4 \mathrm{mmol}, 1.2$ equiv; $2.5 \mathrm{M}$ in $n$-hexane) was added dropwise and the solution was warmed to $0{ }^{\circ} \mathrm{C}$ over $30 \mathrm{~min}$ by exchanging the cooling bath to an ice bath. After cooling back to $-78{ }^{\circ} \mathrm{C}$ (acetone/dry ice), a solution of (-)-camphor $(0.30 \mathrm{~g}, 2.0 \mathrm{mmol}, 1.0$ equiv) in anhydrous THF $(2 \mathrm{~mL})$ was added and the resulting solution was stirred at $-78{ }^{\circ} \mathrm{C}$ for $2 \mathrm{~h}$. Then, $N$-phenylbis(trifluoromethanesulfonimide) (0.92 g, $2.4 \mathrm{mmol}, 1.2$ equiv) was added and the solution was warmed to rt by changing the cooling bath to a water bath. The solution was diluted with $\mathrm{NH}_{4} \mathrm{Cl}$ (sat. aq., $20 \mathrm{~mL}$ ) and EtOAc $(20 \mathrm{~mL})$. The aqueous layer was separated and extracted with EtOAc $(3 \times 20 \mathrm{~mL})$. The combined organic layers were dried $\left(\mathrm{Na}_{2} \mathrm{SO}_{4}\right)$, filtered, and concentrated under reduced pressure. Flash column chromatography $\left(\mathrm{SiO}_{2}, n\right.$-pentane) gave the title compound $\mathbf{S 3}(0.40 \mathrm{~g}, 1.4 \mathrm{mmol}, 72 \%)$ as a colorless oil.

$R_{\mathrm{f}}=0.71\left(\mathrm{SiO}_{2} ; n\right.$-pentane; vis. vanillin).

${ }^{1} \mathrm{H} \mathrm{NMR}\left(500 \mathrm{MHz}, \mathrm{CDCl}_{3}\right) \delta[\mathrm{ppm}]=5.66(\mathrm{~d}, J=3.8 \mathrm{~Hz}, 1 \mathrm{H}), 2.45(\mathrm{t}, J=3.8 \mathrm{~Hz}, 1 \mathrm{H}), 1.93$ (ddt, $J=12.2,8.5,3.7 \mathrm{~Hz}, 1 \mathrm{H}), 1.65(\mathrm{dddd}, J=12.2,8.6,3.7,0.7 \mathrm{~Hz}, 1 \mathrm{H}), 1.33(\mathrm{ddd}, J=12.5,9.2,3.7 \mathrm{~Hz}$, 1H), 1.15 (ddd, $J=12.5,9.1,3.7 \mathrm{~Hz}, 1 \mathrm{H}), 1.03$ (s, 3H), 0.92 (s, 3H), 0.79 (s, 3H).

${ }^{13} \mathrm{C}\left\{{ }^{1} \mathrm{H}\right\} \operatorname{NMR}\left(176 \mathrm{MHz}, \mathrm{CDCl}_{3}\right) \delta[\mathrm{ppm}]=155.4,119.6(\mathrm{q}, J=315.3 \mathrm{~Hz}), 117.8,57.1,54.0,50.3$, 31.0, 25.5, 19.9, 19.1, 9.6. 
${ }^{19} \mathrm{~F} \mathrm{NMR}\left(565 \mathrm{MHz}, \mathrm{CDCl}_{3}\right) \delta[\mathrm{ppm}]=-73.5$.

The spectroscopic data are consistent with those reported in the literature. ${ }^{[6]}$

Note: The title compound is volatile. Concentration at a pressure of less than $100 \mathrm{mbar} / 40^{\circ} \mathrm{C}$ can lead to significant loss of product.

\section{Synthesis of 1-bromo-1'-[(1S, 4S)- 1,7,7-trimethylbicyclo[2.2.1]hept-2-enyl]ferrocene (1g)}

1) $n$-BuLi (1.0 equiv)

$\mathrm{THF},-78^{\circ} \mathrm{C}, 1 \mathrm{~h}$

2) $\mathrm{ZnCl}_{2}$ (1.5 equiv)

$-78^{\circ} \mathrm{C} \rightarrow \mathrm{rt}, 1 \mathrm{~h}$

3) $\mathbf{S 3}$ (1.1 equiv)

$\mathrm{Pd}_{2}(\mathrm{dba})_{3}(1 \mathrm{~mol} \%)$

TFP (6 mol\%),

rt, 18 h, $72 \%$

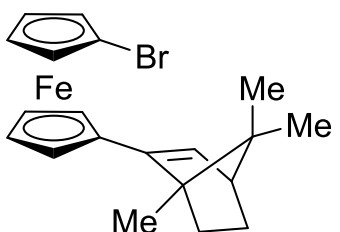

In a dry $100 \mathrm{~mL}$ Schlenk tube, $\mathbf{1 b}(0.44 \mathrm{~g}, 1.3 \mathrm{mmol} 1.0$ equiv) was placed and the flask was evacuated and backfilled with argon $(3 \times)$. Anhydrous THF $(22 \mathrm{~mL})$ was added via syringe and the resulting orange solution was cooled to $-78^{\circ} \mathrm{C}$ (acetone/dry ice). Then, $n$-butyllithium ( $0.51 \mathrm{~mL}, 1.3 \mathrm{mmol}, 1.0$ equiv; $2.5 \mathrm{M}$ in $n$-hexane) was added dropwise over $15 \mathrm{~min}$ and the resulting red solution was stirred at $-78{ }^{\circ} \mathrm{C}$ for $1 \mathrm{~h}$. A solution of $\mathrm{ZnCl}_{2}(0.26 \mathrm{~g}, 1.9 \mathrm{mmol}, 1.5$ equiv) in anhydrous THF ( $2 \mathrm{~mL})$ was added and the resulting yellow solution was stirred at $-78{ }^{\circ} \mathrm{C}$ for $30 \mathrm{~min}$ and then warmed to $\mathrm{rt}$ over $30 \mathrm{~min}$ by removing the cooling bath. A solution of $\mathbf{S 3}(0.40 \mathrm{~g}, 1.4 \mathrm{mmol}, 1.1$ equiv. $)$ in anhydrous THF ( $2 \mathrm{~mL})$, tris(dibenzylideneacetone)dipalladium(0) (12 mg, $13 \mu \mathrm{mol}, 1 \mathrm{~mol} \%)$ and tri(2-furyl)phosphine (18 mg, $77 \mu \mathrm{mol}, 6 \mathrm{~mol} \%$ ) were added in a continuous argon stream. The Schlenk tube was sealed and the orange suspension was stirred at $\mathrm{rt}$ for $18 \mathrm{~h}$. The resulting red solution was diluted with $\mathrm{NH}_{4} \mathrm{Cl}$ (sat. aq., $30 \mathrm{~mL}$ ) and EtOAc $(30 \mathrm{~mL})$. The aqueous layer was separated and extracted with EtOAc $(3 \times 60 \mathrm{~mL})$. The combined organic layers were dried $\left(\mathrm{Na}_{2} \mathrm{SO}_{4}\right)$, filtered, concentrated under reduced pressure, and loaded onto silica. Flash column chromatography $\left(\mathrm{SiO}_{2}, n\right.$-pentane) gave the title compound $\mathbf{1 g}(0.37 \mathrm{~g}$, $0.94 \mathrm{mmol}, 73 \%$ ) essentially pure as an orange solid. An analytically pure sample was obtained by HPLC (Phenomenex 21.2×250 mm RP Gemini NX $5 \mu \mathrm{m} \mathrm{C18;0.7} \mathrm{L/min;} \mathrm{t}_{\mathrm{r}} 8.5 \mathrm{~min}$ ).

$R_{\mathrm{f}}=0.56\left(\mathrm{SiO}_{2} ; n\right.$-pentane; vis. vanillin $)$.

${ }^{1} \mathrm{H}$ NMR $\left(500 \mathrm{MHz}, \mathrm{CDCl}_{3}\right) \delta[\mathrm{ppm}]=6.06(\mathrm{~d}, J=3.3 \mathrm{~Hz}, 1 \mathrm{H}), 4.37-4.28(\mathrm{~m}, 4 \mathrm{H}), 4.25(\mathrm{tdd}, J=3.8$, $2.4,1.9 \mathrm{~Hz}, 2 \mathrm{H}), 4.08(\mathrm{t}, J=1.9 \mathrm{~Hz}, 2 \mathrm{H}), 2.31(\mathrm{t}, J=3.5 \mathrm{~Hz}, 1 \mathrm{H}), 1.90(\mathrm{ddt}, J=12.3,8.7,3.7 \mathrm{~Hz}, 1 \mathrm{H})$, $1.60(\mathrm{ddd}, J=11.8,8.7,3.5 \mathrm{~Hz}, 1 \mathrm{H}), 1.26$ (s, 3H), $1.26-1.14$ (m, 1H), 1.03 (ddd, $J=12.3,9.1,3.6 \mathrm{~Hz}$, $1 \mathrm{H}), 0.91(\mathrm{~s}, 3 \mathrm{H}), 0.82(\mathrm{~s}, 3 \mathrm{H})$.

${ }^{13} \mathrm{C}\left\{{ }^{1} \mathrm{H}\right\} \mathrm{NMR}\left(126 \mathrm{MHz}, \mathrm{CDCl}_{3}\right) \delta[\mathrm{ppm}]=144.7,130.8,84.7,78.0,71.2,71.2,71.0,71.0,69.2,68.6$, $68.4,68.4,56.9,55.2,51.7,32.1,25.8,20.2,19.8,13.4$. 
HRMS (ESI-TOF) m/z calcd. for $\mathrm{C}_{20} \mathrm{H}_{23} \mathrm{BrFe}^{+}\left([\mathrm{M}]^{+}\right)$398.0333; found: 398.0333 .

(S)-1-Iodo-2-[3-(R)-(N,N-dimethylamino)ethyl]ferrocene (1h)

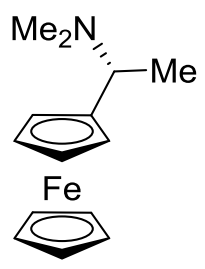

1) $t$-BuLi (1.1 equiv)

$\mathrm{Et}_{2} \mathrm{O}, 0^{\circ} \mathrm{C}, 1 \mathrm{~h}$

2) $I_{2}$ (1.1 equiv)

$\mathrm{Et}_{2} \mathrm{O}, 0{ }^{\circ} \mathrm{C}->\mathrm{rt}, 16 \mathrm{~h}$
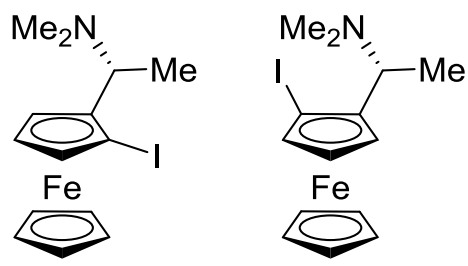

$69 \%(\mathrm{dr}=94: 6)$

This compound was prepared according to a reported procedure. ${ }^{[7]}$

In a dry $25 \mathrm{~mL}$ Schlenk tube, $(R)$ - $N, N$-dimethyl-1-ferrocenylethylamine ( $0.26 \mathrm{~g}, 1.0 \mathrm{mmol}, 1.0$ equiv) was placed. Anhydrous $\mathrm{Et}_{2} \mathrm{O}(6 \mathrm{~mL})$ was added and the resulting solution was cooled to $0{ }^{\circ} \mathrm{C}$ (ice bath). tert-Butyllithium (0.66 mL, $1.1 \mathrm{mmol}, 1.1$ equiv.; $1.7 \mathrm{M}$ in $n$-pentane) was added dropwise and the reaction mixture was stirred at $0{ }^{\circ} \mathrm{C}$ for $1 \mathrm{~h}$. Then, a solution of iodine $(0.31 \mathrm{~g}, 1.2 \mathrm{mmol}, 1.2$ equiv) in $\mathrm{Et}_{2} \mathrm{O}(7.5 \mathrm{~mL})$ was added dropwise. The reaction mixture was slowly warmed to rt. Then, the mixture was diluted with $\mathrm{Na}_{2} \mathrm{~S}_{2} \mathrm{O}_{3}$ (sat. aq., $10 \mathrm{~mL}$ ) and $\mathrm{Et}_{2} \mathrm{O}(10 \mathrm{~mL}$ ), the aqueous layer was separated and extracted with $\mathrm{Et}_{2} \mathrm{O}(3 \times 10 \mathrm{~mL})$. The combined organic layers were washed with $\mathrm{H}_{2} \mathrm{O}(20 \mathrm{~mL}), \mathrm{NaCl}$ (sat. aq., $20 \mathrm{~mL}$ ), dried $\left(\mathrm{MgSO}_{4}\right)$, filtered, and concentrated under reduced pressure. Flash column chromatography $\left(\mathrm{SiO}_{2}, \mathrm{CH}_{2} \mathrm{Cl}_{2} / \mathrm{Et}_{2} \mathrm{O} 1: 1+0.5 \% \mathrm{NEt}_{3}\right)$ gave the title compound $\mathbf{1 h}(0.27 \mathrm{~g}, 0.70 \mathrm{mmol}$, $69 \%$, dr 94:6 [ $\left.\left.{ }^{1} \mathrm{H} \mathrm{NMR}\right]\right)$ as an orange oil.

$\mathrm{R}_{\mathrm{f}}=0.30\left(\mathrm{SiO}_{2}, \mathrm{CH}_{2} \mathrm{Cl}_{2} / \mathrm{Et}_{2} \mathrm{O} 1: 1+0.5 \% \mathrm{NEt}_{3}\right.$, vis. vanillin $)$.

${ }^{1} \mathrm{H}$ NMR $\left(500 \mathrm{MHz}, \mathrm{CDCl}_{3}\right): \delta[\mathrm{ppm}]=4.46(\mathrm{dd}, J=2.5,1.3 \mathrm{~Hz}, 1 \mathrm{H}), 4.24(\mathrm{pt}, J=2.5 \mathrm{~Hz}, 1 \mathrm{H}), 4.15$ $(\mathrm{dd}, J=2.5,1.3 \mathrm{~Hz}, 1 \mathrm{H}), 4.12(\mathrm{~s}, 5 \mathrm{H}), 3.62(\mathrm{q}, J=6.8 \mathrm{~Hz}, 1 \mathrm{H}), 2.14(\mathrm{~s}, 6 \mathrm{H}), 1.50(\mathrm{~d}, J=6.8 \mathrm{~Hz}, 3 \mathrm{H})$.

${ }^{13} \mathrm{C}\left\{{ }^{1} \mathrm{H}\right\} \mathrm{NMR}\left(126 \mathrm{MHz}, \mathrm{CDCl}_{3}\right): \delta[\mathrm{ppm}]=90.3,74.5,71.8,68.3,65.7,57.7,45.6,41.3,16.1$.

The spectroscopic data are consistent with those reported in the literature. ${ }^{[7]}$ 
General Procedure 1 (GP1) for the Sonogashira coupling of 1,1'-diiodoferrocene with various acetylenes

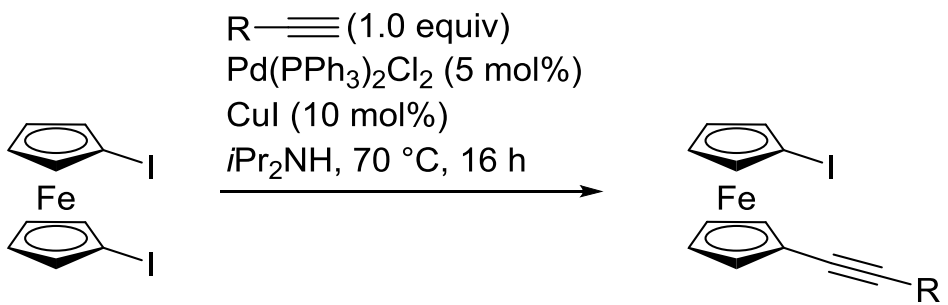

In a dry Schlenk tube, 1c (1.0 equiv) was placed and the flask was evacuated and backfilled with argon $(3 \times)$. Anhydrous $(i-\mathrm{Pr})_{2} \mathrm{NH}($ ca. $0.15 \mathrm{M})$ was added via syringe. To the resulting orange solution, the corresponding acetylene (1.0 equiv), bis(triphenylphosphine)palladium(II) dichloride (5 mol\%) and copper(I) iodide (10 mol\%) were added in a continuous stream of argon. The schlenk tube was sealed and the orange suspension was stirred at $70{ }^{\circ} \mathrm{C}$ for $16 \mathrm{~h}$. After cooling to $\mathrm{rt}$, the red suspension was diluted with EtOAc, filtered over a pad of Celite ${ }^{\circledR}$, and eluted with EtOAc (3x). The filtrate was concentrated and loaded onto Celite ${ }^{\circledR}$. Flash column chromatography gave the ethynyl-substituted ferrocenes $\mathbf{1 i}-\mathbf{1 n}$.

Synthesis of 1-iodo-1'-(trimethylsilylethynyl)ferrocene (1i)

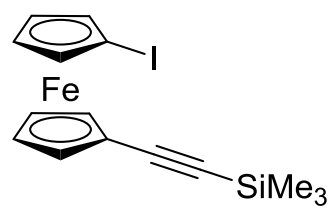

According to GP1, the title compound was prepared from 1c (700 mg, $1.60 \mathrm{mmol}, 1.0$ equiv), $i \mathrm{Pr}_{2} \mathrm{NH}$ (10 mL), trimethylsilylacetylene ( $221 \mu \mathrm{L}, 1.60 \mathrm{mmol}, 1.0$ equiv), bis(triphenylphosphine)palladium(II) dichloride (56.0 mg, $80 \mu \mathrm{mol}, 5 \mathrm{~mol} \%$ ), and copper(I) iodide (30.4 mg, $160 \mu \mathrm{mol}, 10 \mathrm{~mol} \%$ ). Flash column chromatography $\left(\mathrm{SiO}_{2}, n\right.$-pentane/ $\mathrm{CH}_{2} \mathrm{Cl}_{2}$ 100:0 to 50:1 to 10:1) gave the title compound $\mathbf{1 i}$ (299 mg, $733 \mu \mathrm{mol}, 46 \%$ ) as a red oil.

$R_{\mathrm{f}}=0.30\left(\mathrm{SiO}_{2} ; n\right.$-hexane; vis. vanillin $)$.

${ }^{1} \mathrm{H} \mathrm{NMR}\left(700 \mathrm{MHz}, \mathrm{CDCl}_{3}\right) \delta[\mathrm{ppm}]=4.39(\mathrm{dt}, J=8.5,1.9 \mathrm{~Hz}, 4 \mathrm{H}), 4.19(\mathrm{dt}, J=7.9,1.9 \mathrm{~Hz}, 4 \mathrm{H}), 0.24$ $(\mathrm{s}, 9 \mathrm{H})$.

${ }^{13} \mathrm{C}\left\{{ }^{1} \mathrm{H}\right\}$ NMR $\left(176 \mathrm{MHz}, \mathrm{CDCl}_{3}\right) \delta[\mathrm{ppm}]=102.9,92.0,76.7,74.6,72.2,71.4,67.2,40.6,0.4$.

The spectroscopic data are consistent with those reported in the literature. ${ }^{[8]}$ 


\section{Synthesis of 1-iodo-1'-(cyclopropylethynyl)ferrocene (1j)}

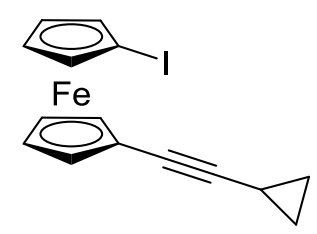

According to GP1, the title compound was prepared from 1c $(0.50 \mathrm{~g}, 1.4 \mathrm{mmol}, 1.0$ equiv), cyclopropylacetylene ( $0.12 \mathrm{~mL}, 1.4 \mathrm{mmol}, 1.2$ equiv), bis(triphenylphosphine)-palladium(II) dichloride (40 mg, $57 \mu \mathrm{mol}, 1.5 \mathrm{~mol} \%$ ), and $\mathrm{CuI}(22 \mathrm{mg}, 0.11 \mathrm{mmol}, 3 \mathrm{~mol} \%)$ in $i \operatorname{Pr}_{2} \mathrm{NH}(6 \mathrm{~mL})$. The mixture was stirred at $50{ }^{\circ} \mathrm{C}$ for $48 \mathrm{~h}$. Flash column chromatography $\left(\mathrm{SiO}_{2}, n\right.$-pentane $/ \mathrm{CH}_{2} \mathrm{Cl}_{2}$ 100:0 to 20:1) gave the title compound $\mathbf{1 j}(0.13 \mathrm{~g}, 0.34 \mathrm{mmol}, 31 \%)$ as an orange oil.

$R_{\mathrm{f}}=0.19\left(\mathrm{SiO}_{2} ; n\right.$-pentane, vis. vanillin $)$.

${ }^{1} \mathrm{H}$ NMR $\left(500 \mathrm{MHz}, \mathrm{CDCl}_{3}\right) \delta[\mathrm{ppm}]=4.38(\mathrm{t}, J=1.8 \mathrm{~Hz}, 2 \mathrm{H}), 4.31(\mathrm{t}, J=1.9 \mathrm{~Hz}, 2 \mathrm{H}), 4.17$ (t, $J=1.8 \mathrm{~Hz}, 2 \mathrm{H}), 4.14(\mathrm{t}, J=1.9 \mathrm{~Hz}, 2 \mathrm{H}), 1.36(\mathrm{tt}, J=8.3,5.0 \mathrm{~Hz}, 1 \mathrm{H}), 0.83(\mathrm{ddt}, J=8.3,5.7,3.0 \mathrm{~Hz}$, 2H), 0.77 (ddd, $J=7.6,5.2,2.8 \mathrm{~Hz}, 2 \mathrm{H}$ ).

${ }^{13} \mathrm{C}\left\{{ }^{1} \mathrm{H}\right\} \mathrm{NMR}\left(126 \mathrm{MHz}, \mathrm{CDCl}_{3}\right) \delta[\mathrm{ppm}]=90.9,76.3,74.1,72.4,71.6,71.0,68.8,41.1,8.7,0.5$.

HRMS (ESI-TOF) m/z calcd. for $\mathrm{C}_{15} \mathrm{H}_{13} \mathrm{FeI}^{+}\left([\mathrm{M}]^{+}\right) 375.9411$; found: 375.9411 .

\section{Synthesis of 1-iodo-1'-(phenylethynyl)ferrocene (1k)}

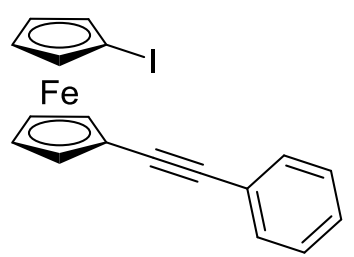

According to GP1, the title compound was prepared from 1c (500 mg, $1.14 \mathrm{mmol}$, 1.0 equiv), phenylacetylene (125 $\mu \mathrm{L}, 1.14 \mathrm{mmol}, 1.0$ equiv), bis(triphenylphosphine)palladium(II) dichloride (40.0 mg, $570 \mu \mathrm{mol}, 5 \mathrm{~mol} \%$ ), and $\mathrm{CuI}(22.0 \mathrm{mg}, 114 \mu \mathrm{mol}, 10 \mathrm{~mol} \%)$ in anhydrous $i \mathrm{Pr}_{2} \mathrm{NH}(10 \mathrm{~mL})$. Flash column chromatography $\left(\mathrm{SiO}_{2}, n\right.$-pentane $/ \mathrm{CH}_{2} \mathrm{Cl}_{2}$ 100:0 to 10:1) followed by recrystallization from $n$-pentane at $-78{ }^{\circ} \mathrm{C}$ gave the title compound $1 \mathbf{k}(208 \mathrm{mg}, 505 \mu \mathrm{mol}, 44 \%)$ as an orange powder. $R_{\mathrm{f}}=0.23\left(\mathrm{SiO}_{2} ; n\right.$-pentane; vis. vanillin).

${ }^{1} \mathrm{H}$ NMR $\left(700 \mathrm{MHz}, \mathrm{CDCl}_{3}\right) \delta[\mathrm{ppm}]=7.52(\mathrm{dd}, J=7.6,2.0 \mathrm{~Hz}, 2 \mathrm{H}), 7.33-7.32(\mathrm{~m}, 3 \mathrm{H}), 4.48(\mathrm{t}$, $J=1.9 \mathrm{~Hz}, 2 \mathrm{H}), 4.45(\mathrm{t}, J=1.8 \mathrm{~Hz}, 2 \mathrm{H}), 4.26(\mathrm{t}, J=1.9 \mathrm{~Hz}, 2 \mathrm{H}), 4.24(\mathrm{t}, J=1.8 \mathrm{~Hz}, 2 \mathrm{H})$.

${ }^{13} \mathrm{C}\left\{{ }^{1} \mathrm{H}\right\}$ NMR $\left(176 \mathrm{MHz}, \mathrm{CDCl}_{3}\right) \delta[\mathrm{ppm}]=131.6,128.4,128.0,123.9,87.1,87.1,76.5,74.2,72.2$, $71.1,67.8,41.3$.

The spectroscopic data are consistent with those reported in the literature. ${ }^{[9]}$ 


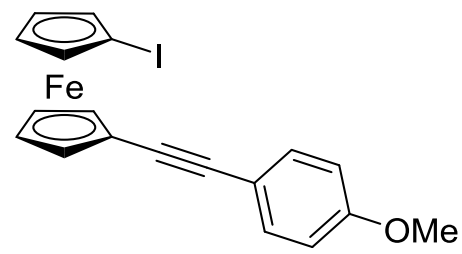

According to GP1, the title compound was prepared from 1c ( $250 \mathrm{mg}, 571 \mu \mathrm{mol}, 1.0$ equiv), 1-ethynyl4-methoxybenzene (75.8 mg, $571 \mu \mathrm{mol}, 1.0$ equiv), bis(triphenylphosphine)-palladium(II) dichloride (20.0 mg, $29.0 \mu \mathrm{mol}, 5 \mathrm{~mol} \%$ ), and $\mathrm{CuI}(10.9 \mathrm{mg}, 57.0 \mu \mathrm{mol}, 10 \mathrm{~mol} \%)$ in anhydrous $i \operatorname{Pr}_{2} \mathrm{NH}(4 \mathrm{~mL})$. Flash column chromatography $\left(\mathrm{SiO}_{2}, n\right.$-pentane/ $\mathrm{CH}_{2} \mathrm{Cl}_{2}$ 8:1) gave the title compound 11 (94.0 $\mathrm{mg}$, $213 \mu \mathrm{mol}, 37 \%)$ as a red solid.

$R_{\mathrm{f}}=0.25\left(\mathrm{SiO}_{2} ; n\right.$-pentane/ $\mathrm{CH}_{2} \mathrm{Cl}_{2} 8: 1 ;$ vis. vanillin $)$.

${ }^{1} \mathrm{H}$ NMR $\left(700 \mathrm{MHz}, \mathrm{CDCl}_{3}\right) \delta[\mathrm{ppm}]=7.46(\mathrm{~d}, J=8.8 \mathrm{~Hz}, 2 \mathrm{H}), 6.86(\mathrm{~d}, J=8.8 \mathrm{~Hz}, 2 \mathrm{H}), 4.45(\mathrm{dt}$, $J=6.9,1.8 \mathrm{~Hz}, 4 \mathrm{H}), 4.23(\mathrm{dt}, J=10.3,1.9 \mathrm{~Hz}, 4 \mathrm{H}), 3.83(\mathrm{~s}, 3 \mathrm{H})$.

${ }^{13} \mathrm{C}\left\{{ }^{1} \mathrm{H}\right\} \mathrm{NMR}\left(176 \mathrm{MHz}, \mathrm{CDCl}_{3}\right) \delta[\mathrm{ppm}]=159.5,133.1,116.1,114.1,86.9,85.5,76.4,74.1,72.0$, 71.0, 68.3, 55.5, 41.4 .

HRMS (ESI-TOF) m/z calcd. for $\mathrm{C}_{19} \mathrm{H}_{16} \mathrm{FeIO}^{+}\left([\mathrm{M}+\mathrm{H}]^{+}\right)$442.9590; found: 442.9605 .

\section{Synthesis of 1-iodo-1'-(1-ethynyl-4-trifluoromethylbenzene)ferrocene (1m)}

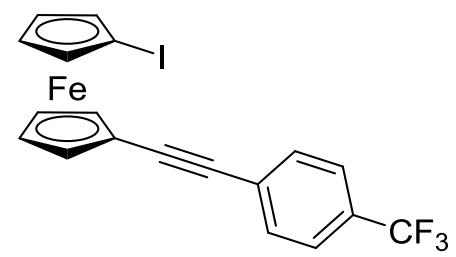

According to GP1, the title compound was prepared from 1c (310 mg, $708 \mu \mathrm{mol}, 1.0$ equiv), 1-ethynyl4-trifluoromethylbenzene (121 mg, $708 \mu \mathrm{mol}, 1.0$ equiv), bis(triphenylphosphine)-palladium(II) dichloride (24.8 mg, $35.0 \mu \mathrm{mol}, 5 \mathrm{~mol} \%)$ and $\mathrm{CuI}(13.5 \mathrm{mg}, 71.0 \mu \mathrm{mol}, 10 \mathrm{~mol} \%)$ in $i \mathrm{Pr}_{2} \mathrm{NH}(10 \mathrm{~mL})$. Flash column chromatography $\left(\mathrm{SiO}_{2}, n\right.$-pentane $/ \mathrm{CH}_{2} \mathrm{Cl}_{2}$ 100:0 to 20:1) gave the title compound $\mathbf{1 m}$ (111 mg, $231 \mu \mathrm{mol}, 33 \%$ ) as a red solid.

$R_{\mathrm{f}}=0.55\left(\mathrm{SiO}_{2} ; n\right.$-pentane, vis. vanillin)

${ }^{1} \mathrm{H}-\mathrm{NMR}\left(500 \mathrm{MHz}, \mathrm{CDCl}_{3}\right): \delta[\mathrm{ppm}]=7.61(\mathrm{~d}, J=8.3 \mathrm{~Hz}, 2 \mathrm{H}), 7.58(\mathrm{~d}, J=7.7 \mathrm{~Hz}, 2 \mathrm{H}), 4.50(\mathrm{pt}$, $J=2.0 \mathrm{~Hz}, 2 \mathrm{H}), 4.46$ (pt, $J=2.0 \mathrm{~Hz}, 2 \mathrm{H}), 4.30$ (pt, $J=2.0 \mathrm{~Hz}, 2 \mathrm{H}), 4.24$ (pt, $J=2.0 \mathrm{~Hz}, 2 \mathrm{H}$ ).

${ }^{13} \mathrm{C}\left\{{ }^{1} \mathrm{H}\right\} \operatorname{NMR}\left(176 \mathrm{MHz}, \mathrm{CDCl}_{3}\right) \delta[\mathrm{ppm}]=131.7,129.6(\mathrm{q}, J=32.5 \mathrm{~Hz}), 127.8,125.4(\mathrm{q}, J=3.8 \mathrm{~Hz})$, $124.2(J=272.2 \mathrm{~Hz}), 90.2,85.9,76.6,74.4,72.4,71.0,70.2,66.9$.

${ }^{19} \mathrm{~F} \mathrm{NMR}\left(471 \mathrm{MHz}, \mathrm{CDCl}_{3}\right): \delta[\mathrm{ppm}]=62.6$.

HRMS (ESI-TOF) m/z calcd. for $\mathrm{C}_{19} \mathrm{H}_{12} \mathrm{~F}_{3} \mathrm{FeI}^{+}$([M] $]^{+}$) 479.9285; found: 479.9290 . 


\section{Synthesis of 1-iodo-1'-(pyridylethynyl)ferrocene (1n)}

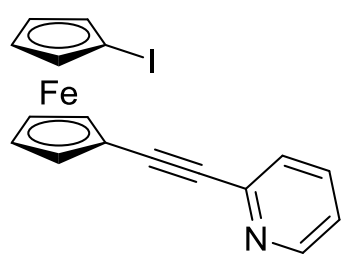

According to GP1, the title compound was prepared from 1c ( $250 \mathrm{mg}, 571 \mu \mathrm{mol}, 1.0$ equiv), 2ethynylpyridine (63.3 $\mu \mathrm{L}, 58.9 \mathrm{mg}, 571 \mu \mathrm{mol}, 1.0$ equiv), bis(triphenylphosphine)-palladium(II) dichloride (20.0 mg, $29.0 \mu \mathrm{mol}, 5 \mathrm{~mol} \%)$, and $\mathrm{CuI}(10.9 \mathrm{mg}, 57.0 \mu \mathrm{mol}, 10 \mathrm{~mol} \%)$ in anhydrous $i \operatorname{Pr}_{2} \mathrm{NH}$ $(4 \mathrm{~mL})$. Flash column chromatography $\left(\mathrm{SiO}_{2}, n\right.$-hexane/EtOAc 5:1) gave the title compound 1n (101 mg, $245 \mu \mathrm{mol}, 43 \%)$ as a red solid.

$R_{\mathrm{f}}=0.28\left(\mathrm{SiO}_{2} ; n\right.$-hexane/EtOAc 5:1; vis. vanillin $)$.

${ }^{1} \mathrm{H}$ NMR $\left(500 \mathrm{MHz}, \mathrm{CDCl}_{3}\right) \delta[\mathrm{ppm}]=8.65-8.55(\mathrm{~m}, 1 \mathrm{H}), 7.65(\mathrm{td}, J=7.7,1.7 \mathrm{~Hz}, 1 \mathrm{H}), 7.50(\mathrm{~d}$, $J=7.8 \mathrm{~Hz}, 1 \mathrm{H}), 7.22(\mathrm{dd}, J=7.8,4.7 \mathrm{~Hz}, 1 \mathrm{H}), 4.55(\mathrm{t}, J=1.9 \mathrm{~Hz}, 2 \mathrm{H}), 4.46(\mathrm{t}, J=1.9 \mathrm{~Hz}, 2 \mathrm{H}), 4.28(\mathrm{t}$, $J=1.9 \mathrm{~Hz}, 2 \mathrm{H}), 4.25(\mathrm{t}, J=1.9 \mathrm{~Hz}, 2 \mathrm{H})$.

${ }^{13} \mathrm{C}\left\{{ }^{1} \mathrm{H}\right\} \mathrm{NMR}\left(126 \mathrm{MHz}, \mathrm{CDCl}_{3}\right) \delta[\mathrm{ppm}]=150.1,144.0,136.2,127.0,122.5,88.0,86.6,76.5,74.5$, $72.8,71.4,66.2,40.9$.

The spectroscopic data are consistent with those reported in the literature. ${ }^{[10]}$ 


\subsection{Synthesis of Ferrocenyl Azides in Batch}

\section{General Procedure 2 (GP2) for the synthesis of ferrocenyl azides in batch}

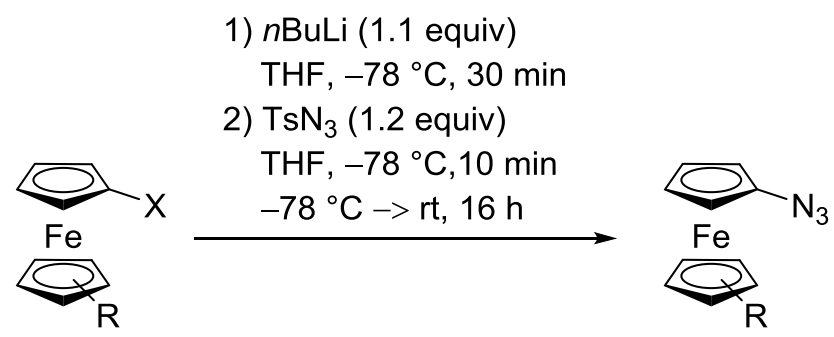

In a dry Schlenk tube, the ferrocenyl halide (1.0 equiv) was placed and the Schlenk tube was evacuated and backfilled with argon $(3 \times)$. THF $(0.05-0.32 \mathrm{M})$ was added and the resulting solution was cooled to $-78^{\circ} \mathrm{C}$ (acetone/dry ice). $n$-Butyllithium (1.1 equiv) was added dropwise and the mixture was stirred for $30 \mathrm{~min}$. Then, tosyl azide (1.2 equiv) was added dropwise at $-78^{\circ} \mathrm{C}$ and the resulting dark red solution was stirred at this temperature for $1 \mathrm{~h}$. The cooling bath was removed, the Schlenk tube was wrapped in aluminum foil and the mixture was stirred for $16 \mathrm{~h}$ at rt. After exposure to air, $\mathrm{NaHCO}_{3}$ (sat. aq.) and EtOAc were added. The aqueous layer was separated and extracted with EtOAc. The combined organic layers were dried $\left(\mathrm{Na}_{2} \mathrm{SO}_{4}\right)$, filtered, and concentrated under reduced pressure. Gravity column chromatography (basic $\mathrm{Al}_{2} \mathrm{O}_{3}$, activity grade $\mathrm{I}+5 \mathrm{wt} \% \mathrm{H}_{2} \mathrm{O}$ ) gave the ferrocenyl azide. If necessary, further purification was performed by recrystallization from $n$-pentane at $-78{ }^{\circ} \mathrm{C}$.

Note 1: Drying with $\mathrm{MgSO}_{4}$ instead of $\mathrm{Na}_{2} \mathrm{SO}_{4}$ gave lower yields.

Note 2: For column chromatography, the crude product was suspended in a small amount of the eluent and the resulting suspension was directly loaded onto the column using a Pasteur pipette.

Note 3: In some cases, flash column chromatography was possible, but in general, gravity column chromatography gave better separations. 


\section{Graphical Guide for the Synthesis of Ferrocenyl Azides in Batch}

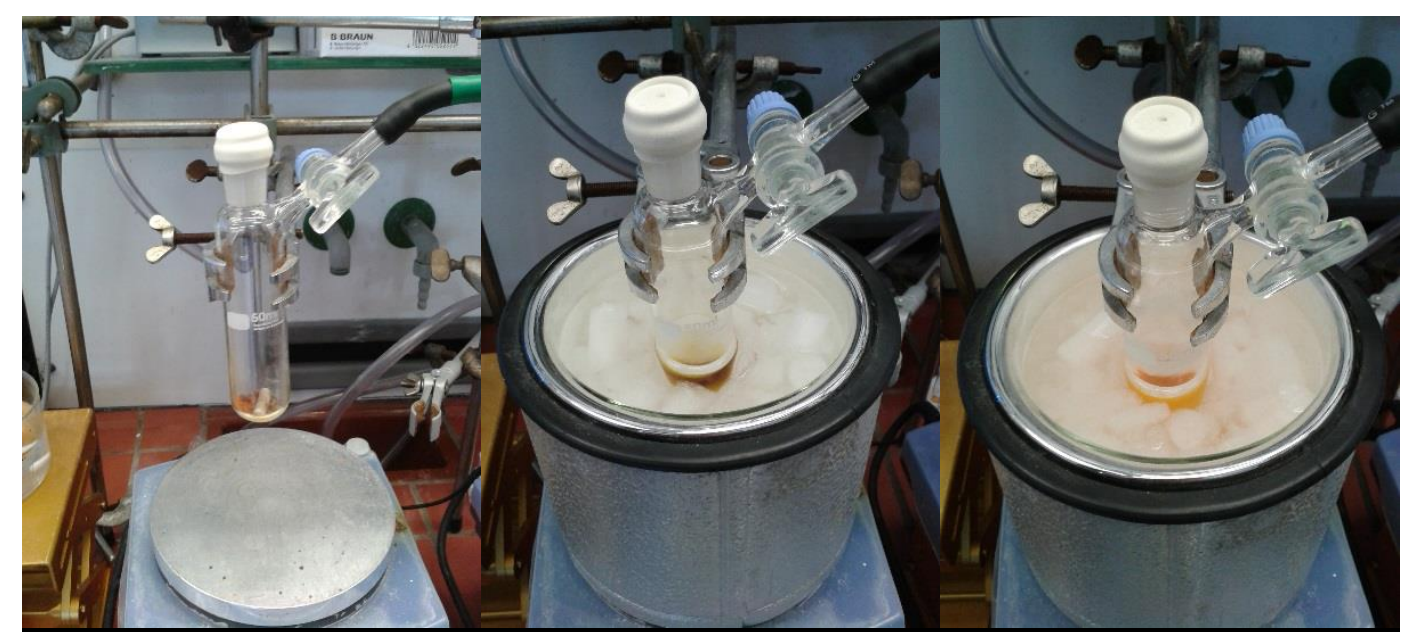

Left: Schlenk tube with ferrocenyl iodide. Center: Solution of ferrocenyl iodide in tetrahydrofuran at $-78{ }^{\circ} \mathrm{C}$. Right: Reaction mixture after adding $n$-butyllithium.

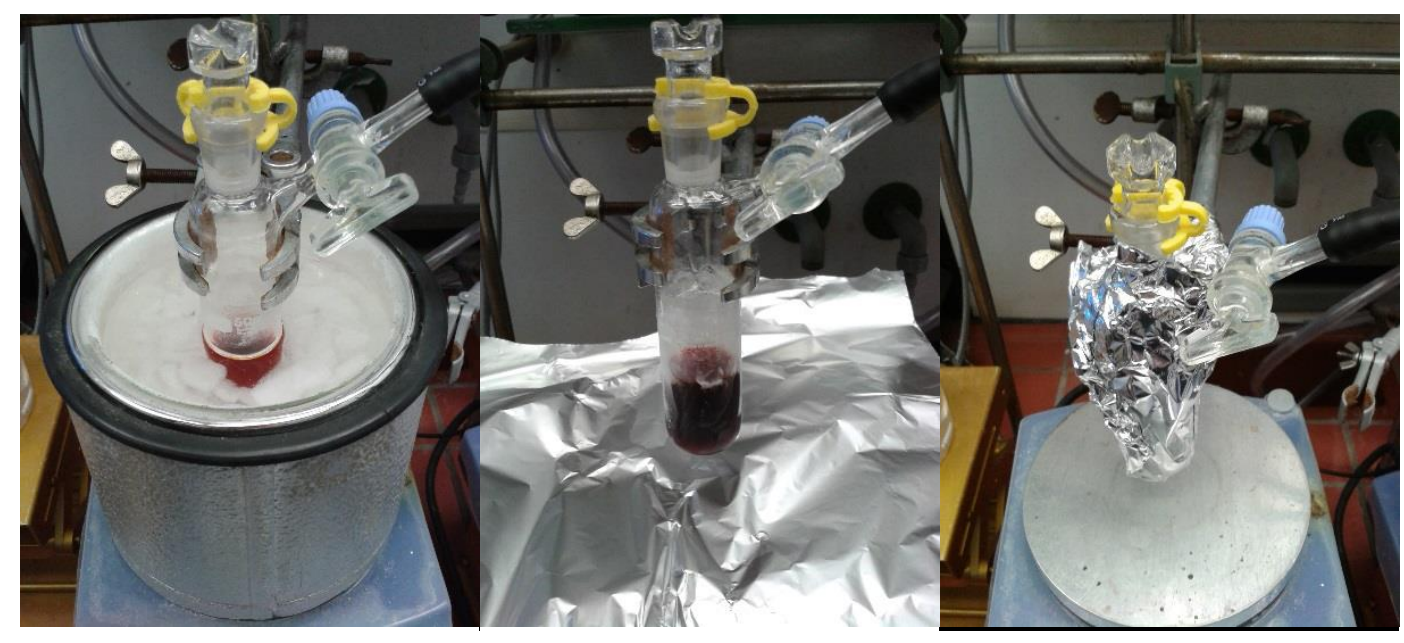

Left: Reaction mixture after adding tosyl azide. Center: Reaction mixture after removing the cold bath. Right: Reaction mixture stirred under exclusion of light. 

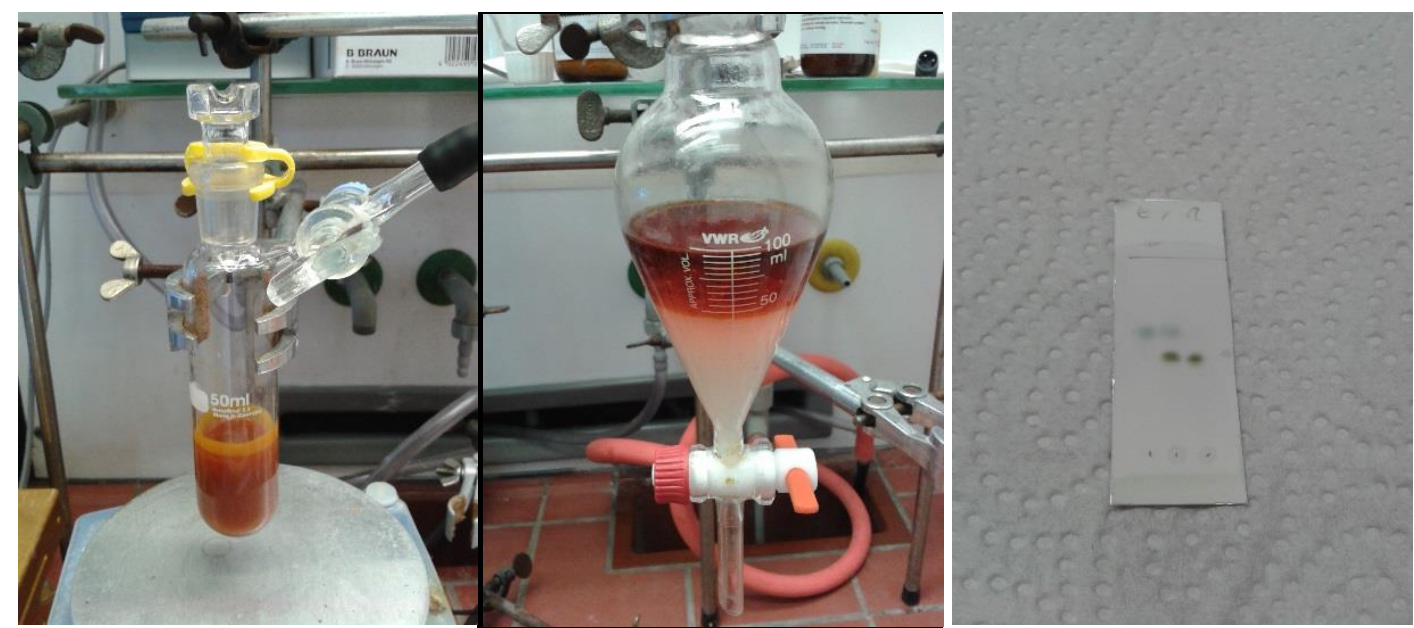

Left: Reaction mixture after stirring at room temperature over night. Center: Aqueous work-up of the reaction mixture. Right: TLC of the reaction mixture after aqueous workup (left lane: starting material, middle lane: co-spot; right lane: crude product; vis. vanillin).

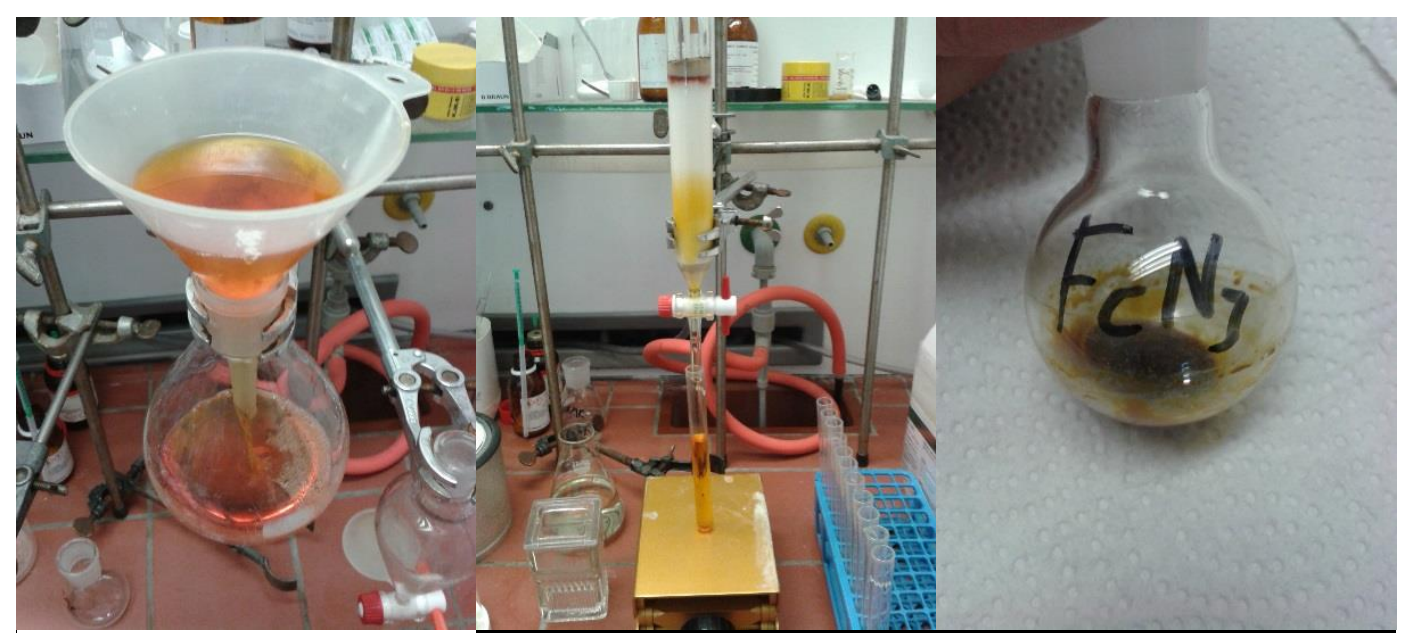

Left: Removal of the drying agent by filtration. Center: Gravity column chromatography of the crude product. Right: Pure product. 
Synthesis of Azidoferrocene (2a)

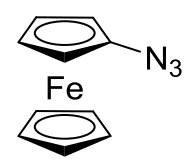

According to GP2, the title compound was prepared from 1a $(0.50 \mathrm{~g}, 1.6 \mathrm{mmol}, 1.0$ equiv $), n$ butyllithium ( $0.71 \mathrm{~mL}, 1.8 \mathrm{mmol}, 1.1$ equiv; $2.5 \mathrm{M}$ in $n$-hexane), and tosyl azide ( $0.77 \mathrm{~mL}, 1.9 \mathrm{mmol}$, 2.5 equiv; $0.75 \mathrm{M}$ in THF) in THF ( $10 \mathrm{~mL}$ ). Gravity column chromatography (basic $\mathrm{Al}_{2} \mathrm{O}_{3}$, activity grade $\mathrm{I}+5 \mathrm{wt} \% \mathrm{H}_{2} \mathrm{O} ; n$-pentane) gave the title compound $2 \mathrm{a}(0.35 \mathrm{~g}, 1.5 \mathrm{mmol}, 95 \%)$ as a red oil that solidified upon standing at $-18^{\circ} \mathrm{C}$.

$R_{\mathrm{f}}=0.41\left(\mathrm{SiO}_{2}, n\right.$-hexane, vis. vanillin).

${ }^{1} \mathrm{H} \mathrm{NMR}\left(400 \mathrm{MHz}, \mathrm{CDCl}_{3}\right) \delta[\mathrm{ppm}]=4.28(\mathrm{~s}, 5 \mathrm{H}), 4.26(\mathrm{t}, J=1.9 \mathrm{~Hz}, 2 \mathrm{H}), 4.04(\mathrm{t}, J=1.9 \mathrm{~Hz}, 2 \mathrm{H})$.

${ }^{13} \mathrm{C}\left\{{ }^{1} \mathrm{H}\right\}$ NMR $\left(100 \mathrm{MHz}, \mathrm{CDCl}_{3}\right) \delta[\mathrm{ppm}]=99.3,69.3,65.4,60.7$.

FT-IR (neat) $\tilde{v}\left[\mathrm{~cm}^{-1}\right]=3109(\mathrm{w}), 3090(\mathrm{w}), 2323(\mathrm{~m}), 2196$ (m), 2113 (s), 2107 (m), 1712 (w), 1451 (s), 1408 (m), 1373 (m), 1350 (m), 1282 (s), 1222 (m), 1104 (s), 1058 (m), 1021 (m), 1000 (s), 915 (m), $892(\mathrm{~m}), 850(\mathrm{~m}), 843(\mathrm{~m}), 819(\mathrm{~s}), 738$ (m), 618 (w), $590(\mathrm{w})$.

HRMS (ESI-TOF) m/z calcd. for $\mathrm{C}_{10} \mathrm{H}_{9} \mathrm{FeN}_{3}{ }^{+}\left([\mathrm{M}]^{+}\right)$227.0146; found: 227.0136 .

Synthesis of 1-azido-1'-bromoferrocene (2b)

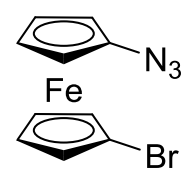

According to GP1, to a solution of $\mathbf{1 b}$ (300 $\mathrm{mg}, 0.873 \mathrm{mmol}, 1.0$ equiv) in anhydrous THF (15 mL), $n$ butyllithium ( $0.349 \mathrm{~mL}, 0.873 \mathrm{mmol}, 1.0$ equiv; $2.5 \mathrm{M}$ in $n$-hexane) was added dropwise over $10 \mathrm{~min}$ at $-78^{\circ} \mathrm{C}$ (acetone/dry ice). During the addition and for another $1 \mathrm{~h}$, the reaction mixture was carefully maintained below $-70{ }^{\circ} \mathrm{C}$ while 1 -lithio-1'-bromoferrocene partially precipitated. Then, tosyl azide $\left(1.40 \mathrm{~mL}, 1.05 \mathrm{mmol}, 1.2\right.$ equiv; $0.75 \mathrm{M}$ in THF) was added dropwise at $-78{ }^{\circ} \mathrm{C}$ over $10 \mathrm{~min}$ and the resulting dark red solution was stirred at this temperature for $1 \mathrm{~h}$. The cooling bath was removed, the Schlenk tube was wrapped in aluminum foil and the mixture was stirred for $16 \mathrm{~h}$ at $\mathrm{rt}$. The mixture was diluted with $\mathrm{NaHCO}_{3}$ (sat. aq., $50 \mathrm{~mL}$ ) and EtOAc $(40 \mathrm{~mL}$ ) and the aqueous layer was separated and extracted with EtOAc $(2 \times 50 \mathrm{~mL})$. The combined organic layers were dried $\left(\mathrm{Na}_{2} \mathrm{SO}_{4}\right)$, filtered, and concentrated under reduced pressure. Gravity column chromatography (basic $\mathrm{Al}_{2} \mathrm{O}_{3}$, activity grade $\mathrm{I}+5$ wt $\% \mathrm{H}_{2} \mathrm{O} ; n$-pentane) gave the title compound $\mathbf{2 b}(242 \mathrm{mg}, 0.791 \mathrm{mmol}, 91 \%)$ as a red oil that solidified upon standing.

$R_{\mathrm{f}}=0.32\left(\mathrm{SiO}_{2}, n\right.$-pentane, vis. vanillin $)$. 
${ }^{1} \mathrm{H} \mathrm{NMR}\left(500 \mathrm{MHz}, \mathrm{CDCl}_{3}\right) \delta[\mathrm{ppm}]=4.52(\mathrm{t}, J=1.9 \mathrm{~Hz}, 2 \mathrm{H}), 4.27(\mathrm{t}, J=2.0 \mathrm{~Hz}, 2 \mathrm{H}), 4.20(\mathrm{t}$, $J=1.9 \mathrm{~Hz}, 2 \mathrm{H}), 4.12(\mathrm{t}, J=2.0 \mathrm{~Hz}, 2 \mathrm{H})$.

${ }^{13} \mathrm{C}\left\{{ }^{1} \mathrm{H}\right\} \mathrm{NMR}\left(126 \mathrm{MHz}, \mathrm{CDCl}_{3}\right) \delta[\mathrm{ppm}]=100.7,78.7,71.5,68.3,68.0,62.9$.

FT-IR (neat) $\tilde{v}\left[\mathrm{~cm}^{-1}\right]=2924(\mathrm{~s}), 2854(\mathrm{~s}), 2111(\mathrm{~s}), 1716(\mathrm{w}), 1459(\mathrm{~m}), 1410(\mathrm{w}), 1374(\mathrm{w}), 1287(\mathrm{~m})$, 1260 (w), 1153 (w), 1098 (w), 1022 (m), 918 (w), 873 (m), 807 (m), 741 (w), 699 (w).

HRMS (ESI-TOF) m/z calcd. for $\mathrm{C}_{10} \mathrm{H}_{8} \mathrm{BrFeN}_{3}{ }^{+}\left([\mathrm{M}]^{+}\right) 304.9251$; found: 304.9243 .

\section{Synthesis of 1-azido-1'-iodoferrocene (2c)}

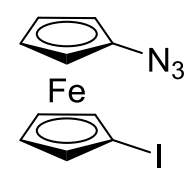

According to GP2, the title compound was prepared from 1c (131 mg, $299 \mu \mathrm{mol}, 1.0$ equiv), $n$ butyllithium ( $110 \mu \mathrm{L}, 299 \mu \mathrm{mol}, 1.0$ equiv; $2.7 \mathrm{M}$ in $n$-hexane), and tosyl azide (142 $\mu \mathrm{L}, 359 \mu \mathrm{mol}$, 1.2 equiv; $2.5 \mathrm{M}$ in THF) in THF (5 mL). Gravity column chromatography (basic $\mathrm{Al}_{2} \mathrm{O}_{3}$, activity grade $\mathrm{I}+5 \mathrm{wt} \% \mathrm{H}_{2} \mathrm{O} ; n$-pentane) gave the title compound $2 \mathrm{c}(27.0 \mathrm{mg}, 77.0 \mu \mathrm{mol}, 26 \%)$ as a brown oil. $R_{\mathrm{f}}=0.39\left(\mathrm{SiO}_{2}, n\right.$-pentane, vis. vanillin $)$.

${ }^{1} \mathrm{H} \mathrm{NMR}\left(400 \mathrm{MHz}, \mathrm{CDCl}_{3}\right) \delta[\mathrm{ppm}]=4.51(\mathrm{t}, J=1.8 \mathrm{~Hz}, 2 \mathrm{H}), 4.25(\mathrm{t}, J=1.8 \mathrm{~Hz}, 2 \mathrm{H}), 4.22(\mathrm{t}$, $J=2.0 \mathrm{~Hz}, 2 \mathrm{H}), 4.08(\mathrm{t}, J=2.0 \mathrm{~Hz}, 2 \mathrm{H})$.

${ }^{13} \mathrm{C}\left\{{ }^{1} \mathrm{H}\right\} \mathrm{NMR}\left(100 \mathrm{MHz}, \mathrm{CDCl}_{3}\right) \delta[\mathrm{ppm}]=100.7,75.9,70.0,68.5,63.3,40.9$.

FT-IR (neat) $\tilde{v}\left[\mathrm{~cm}^{-1}\right]=3098(\mathrm{w}), 2961(\mathrm{w}), 2208(\mathrm{w}), 2107(\mathrm{~s}), 1631(\mathrm{w}), 1457(\mathrm{w}), 1403(2), 1378(\mathrm{~m})$, 1344 (s), 1285 (w), 1261 (m), 1143 (m), 1105 (w), 1051 (w), 1020 (s), 916 (w), 864 (s), 805 (s), 585 (w).

HRMS (ESI-TOF) m/z calcd. for $\mathrm{C}_{10} \mathrm{H}_{8} \mathrm{FeIN}_{3}{ }^{+}\left([\mathrm{M}]^{+}\right)$352.9112; found: 352.9121 .

\section{Synthesis of 1,1 '-diazidoferrocene (2d)}

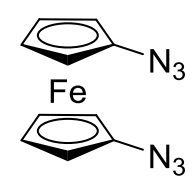

According to GP2, the title compound was prepared from $\mathbf{1 b}$ (200 mg, $0.582 \mathrm{mmol}, 1.0$ equiv), $n$ butyllithium $(0.512 \mathrm{~mL}, 1.28 \mathrm{mmol}, 2.2$ equiv; $2.5 \mathrm{M}$ in $n$-hexane), and tosyl azide $(1.81 \mathrm{~mL}$, $1.34 \mathrm{mmol}, 2.3$ equiv; $0.75 \mathrm{M}$ in THF) in THF ( $5 \mathrm{~mL}$ ). Gravity column chromatography (basic $\mathrm{Al}_{2} \mathrm{O}_{3}$, activity grade $\mathrm{I}+5 \mathrm{wt} \% \mathrm{H}_{2} \mathrm{O}$; $n$-pentane) gave the title compound $\mathbf{2 d}(147 \mathrm{mg}, 0.548 \mathrm{mmol}, 94 \%)$ as an orange oil that solidified upon standing.

$R_{\mathrm{f}}=0.29\left(\mathrm{SiO}_{2}, n\right.$-hexane, vis. vanillin).

${ }^{1} \mathrm{H}$ NMR $\left(700 \mathrm{MHz}, \mathrm{CDCl}_{3}\right) \delta[\mathrm{ppm}]=4.35(\mathrm{t}, J=2.0 \mathrm{~Hz}, 4 \mathrm{H}), 4.15(\mathrm{t}, J=2.0 \mathrm{~Hz}, 4 \mathrm{H})$. 
${ }^{13} \mathrm{C}\left\{{ }^{1} \mathrm{H}\right\}$ NMR $\left(176 \mathrm{MHz}, \mathrm{CDCl}_{3}\right) \delta[\mathrm{ppm}]=100.5,66.5,61.6$.

The spectroscopic data are consistent with those reported in the literature. ${ }^{[1]}$

Synthesis of 1-azido-1'-ferrocenylcarboxylic acid tert-butyl ester (2e)

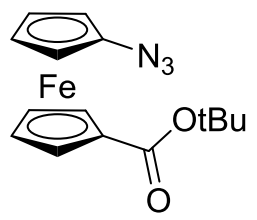

According to GP2, the title compound was prepared from 1e (200 mg, $548 \mu \mathrm{mol}, 1.0$ equiv), $n$ butyllithium ( $241 \mu \mathrm{L}, 603 \mu \mathrm{mol}, 1.1$ equiv; $2.5 \mathrm{M}$ in $n$-hexane) and tosyl azide ( $877 \mu \mathrm{L}, 657 \mu \mathrm{mol}$, 1.2 equiv; $0.75 \mathrm{M}$ in THF) in THF ( $5 \mathrm{~mL}$ ). Gravity column chromatography (basic $\mathrm{Al}_{2} \mathrm{O}_{3}$, activity grade $\mathrm{I}+5 \mathrm{wt} \% \mathrm{H}_{2} \mathrm{O} ; n$-pentane/ $\left.\mathrm{Et}_{2} \mathrm{O} 20: 1\right)$ gave the title compound $2 \mathrm{e}(136 \mathrm{mg}, 416 \mu \mathrm{mol}, 76 \%)$ as a red oil. $R_{\mathrm{f}}=0.40\left(\mathrm{SiO}_{2}, n\right.$-pentane $/ \mathrm{Et}_{2} \mathrm{O}=20: 1$, vis. vanillin $)$.

${ }^{1} \mathrm{H} \mathrm{NMR}\left(400 \mathrm{MHz}, \mathrm{CDCl}_{3}\right) \delta[\mathrm{ppm}]=4.83(\mathrm{t}, J=2.0 \mathrm{~Hz}, 2 \mathrm{H}), 4.43(\mathrm{t}, J=2.0 \mathrm{~Hz}, 2 \mathrm{H}), 4.25(\mathrm{t}$, $J=2.0 \mathrm{~Hz}, 2 \mathrm{H}), 4.07(\mathrm{t}, J=1.9 \mathrm{~Hz}, 2 \mathrm{H}), 1.56(\mathrm{~s}, 9 \mathrm{H})$.

${ }^{13} \mathrm{C}\left\{{ }^{1} \mathrm{H}\right\} \mathrm{NMR}\left(100 \mathrm{MHz}, \mathrm{CDCl}_{3}\right) \delta[\mathrm{ppm}]=170.2,100.6,80.5,74.9,72.0,71.2,67.0,61.8,28.4$.

FT-IR (neat) $\tilde{v}\left[\mathrm{~cm}^{-1}\right]=2976(\mathrm{w}), 2927(\mathrm{w}), 2854(\mathrm{w}), 2443(\mathrm{w}), 2319(\mathrm{w}), 2204(\mathrm{w}), 2111(\mathrm{~s}), 1709(\mathrm{~s})$, 1458 (s), 1393 (w), 1367 (m), 1288 (s), 1256 (w), 1181 (w), 1137 (s), 1025 (w), 917 (w), 814 (w), 778 (w), $741(w)$.

HRMS (ESI): $\mathrm{m} / \mathrm{z}$ calcd. for $\mathrm{C}_{15} \mathrm{H}_{16} \mathrm{FeN}_{3} \mathrm{NaO}_{2}{ }^{+}\left([\mathrm{M}+\mathrm{Na}]^{+}\right)$350.0562; found: 350.0566 .

\section{Synthesis of 1-azido-1'-(1H-benzo[d][1,2,3]triazole)ferrocene (2f)}

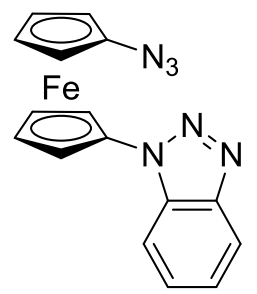

According to GP2, the title compound was prepared from if (39 mg, $0.10 \mathrm{mmol}, 1.0$ equiv), $n$ butyllithium ( $49 \mu \mathrm{L}, 0.12 \mathrm{mmol}, 1.2$ equiv.; $2.5 \mathrm{M}$ in $n$-hexane), and tosyl azide ( $50 \mu \mathrm{L}, 0.12 \mathrm{mmol}$, 1.2 equiv.; $2.5 \mathrm{M}$ in THF) in anhydrous THF ( $2 \mathrm{~mL}$ ). Gravity column chromatography (basic $\mathrm{Al}_{2} \mathrm{O}_{3}$, activity grade $\mathrm{I}+5 \mathrm{wt} \% \mathrm{H}_{2} \mathrm{O} ; n$-hexane/EtOAc $4: 1$ ) followed by recrystallization from $n$-pentane at $-78{ }^{\circ} \mathrm{C}$ gave the title compound $\mathbf{2 f}(24 \mathrm{mg}, 69 \mu \mathrm{mol}, 68 \%)$ as a yellow solid. $R_{\mathrm{f}}=0.30\left(\mathrm{SiO}_{2}, n\right.$-hexane/EtOAc 4:1). 
${ }^{1} \mathrm{H}$ NMR $\left(400 \mathrm{MHz}, \mathrm{CDCl}_{3}\right) \delta[\mathrm{ppm}]=8.11(\mathrm{~d}, J=8.4 \mathrm{~Hz}, 1 \mathrm{H}), 7.81(\mathrm{~d}, J=8.4 \mathrm{~Hz}, 1 \mathrm{H}), 7.57(\mathrm{t}$, $J=7.7 \mathrm{~Hz}, 1 \mathrm{H}), 7.42(\mathrm{t}, J=7.7 \mathrm{~Hz}, 1 \mathrm{H}), 5.08(\mathrm{t}, J=2.0 \mathrm{~Hz}, 2 \mathrm{H}), 4.47(\mathrm{t}, J=2.0 \mathrm{~Hz}, 2 \mathrm{H}), 4.33(\mathrm{t}$, $J=2.0 \mathrm{~Hz}, 2 \mathrm{H}), 4.15(\mathrm{t}, J=2.0 \mathrm{~Hz}, 2 \mathrm{H})$.

${ }^{13} \mathrm{C}\left\{{ }^{1} \mathrm{H}\right\} \operatorname{NMR}\left(100 \mathrm{MHz}, \mathrm{CDCl}_{3}\right) \delta[\mathrm{ppm}]=146.5,133.0,128.0,124.4,120.5,110.7,101.0,94.8,67.9$, 67.4, 63.6, 62.2.

FT-IR (neat) $\tilde{v}\left[\mathrm{~cm}^{-1}\right]=3126(\mathrm{w}), 3104(\mathrm{w}), 3074(\mathrm{w}), 2363(\mathrm{w}), 2333(\mathrm{w}), 2103(\mathrm{~s}), 2031(\mathrm{~m}), 1610$ (w), $1588(\mathrm{w}), 1515(\mathrm{~m}), 1462(\mathrm{~m}), 1450(\mathrm{~m}), 1372(\mathrm{w}), 1297(\mathrm{~m}), 1282(\mathrm{~m}), 1250(\mathrm{w}), 1219(\mathrm{w}), 1204$ (w), 1168 (w), 1147 (w), 1125 (w), 1074 (m), 1058 (m), 1029 (m), 992 (w), 924 (m), 917 (m), 868 (m), $885(\mathrm{~m}), 831(\mathrm{w}), 807(\mathrm{w}), 781(\mathrm{w}), 744(\mathrm{~m})$.

HRMS (ESI): $\mathrm{m} / \mathrm{z}$ calcd. for $\mathrm{C}_{16} \mathrm{H}_{12} \mathrm{FeN}_{6}{ }^{+}\left([\mathrm{M}]^{+}\right) 344.0473$; found: 344.0467 .

\section{Synthesis of 1-azido-1'-[(1S, 4S)- 1,7,7-trimethylbicyclo[2.2.1]hept-2-enyl]ferrocene (2g)}

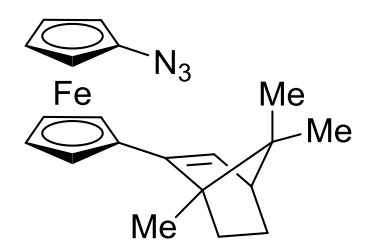

According to GP2, the title compound was prepared from $\mathbf{1 g}$ (200 mg, $501 \mu \mathrm{mol}, 1.0$ equiv), $n$ butyllithium ( $220 \mu \mathrm{L}, 551 \mu$ mol, 1.1 equiv.; $2.5 \mathrm{M}$ in $n$-hexane), and tosyl azide ( $802 \mu \mathrm{L}, 601 \mu \mathrm{mol}$, 1.2 equiv.; $0.75 \mathrm{M}$ in THF) in anhydrous THF $\left(5 \mathrm{~mL}\right.$ ). Gravity column chromatography (basic $\mathrm{Al}_{2} \mathrm{O}_{3}$, activity grade $\mathrm{I}+5 \mathrm{wt} \% \mathrm{H}_{2} \mathrm{O} ; n$-pentane) gave the title compound $\mathbf{2 g}(125 \mathrm{mg}, 346 \mu \mathrm{mol}, 69 \%)$ as an orange oil.

$R_{\mathrm{f}}=0.42\left(\mathrm{SiO}_{2} ; n\right.$-pentane; vis. vanillin).

${ }^{1} \mathrm{H}$ NMR $\left(400 \mathrm{MHz}, \mathrm{CDCl}_{3}\right) \delta[\mathrm{ppm}]=6.04(\mathrm{~d}, J=3.3 \mathrm{~Hz}, 1 \mathrm{H}), 4.39(\mathrm{dq}, J=11.2,1.8 \mathrm{~Hz}, 2 \mathrm{H}), 4.32$ $(\mathrm{t}, J=1.9 \mathrm{~Hz}, 2 \mathrm{H}), 4.18(\mathrm{dq}, J=8.8,1.8 \mathrm{~Hz}, 2 \mathrm{H}), 4.01(\mathrm{t}, J=2.0 \mathrm{~Hz}, 2 \mathrm{H}), 2.30(\mathrm{t}, J=3.5 \mathrm{~Hz}, 1 \mathrm{H}), 1.88$ (ddt, $J=12.2,8.7,3.7 \mathrm{~Hz}, 1 \mathrm{H}), 1.58(\mathrm{ddd}, J=12.0,8.7,3.5 \mathrm{~Hz}, 1 \mathrm{H}), 1.25$ (s, 3H), 1.17 (ddd, $J=12.1$, 9.1, 3.6 Hz, 1H), 1.01 (ddd, $J=12.3,9.1,3.5 \mathrm{~Hz}, 1 \mathrm{H}), 0.89$ (s, 3H), 0.80 (s, 3H).

${ }^{13} \mathrm{C}\left\{{ }^{1} \mathrm{H}\right\} \mathrm{NMR}\left(100 \mathrm{MHz}, \mathrm{CDCl}_{3}\right) \delta[\mathrm{ppm}]=144.8,130.7,99.5,84.5,77.4,69.2,69.1,67.9,66.9,66.6$, 61.6, 61.5, 56.9, 55.2, 51.7, 32.2, 25.8, 20.1, 19.8, 13.3 .

FT-IR (neat) $\tilde{v}\left[\mathrm{~cm}^{-1}\right]=2949(\mathrm{~m}), 2870(\mathrm{~m}), 2104(\mathrm{~s}), 2024(\mathrm{w}), 1456(\mathrm{~s}), 1384(\mathrm{~m}), 1372(\mathrm{~m}), 1363$ (m), 1336 (w), 1285 (m), 1221 (w), 1163 (m), 1132 (w), 1105 (m), 1065 (m), 1023 (m), 917 (m), 878 (m), $818(\mathrm{~s}), 804(\mathrm{~s}), 740(\mathrm{~m}), 708(\mathrm{w}), 699(\mathrm{w})$.

HRMS (ESI): $\mathrm{m} / \mathrm{z}$ calcd. for $\mathrm{C}_{20} \mathrm{H}_{23} \mathrm{FeN}_{3}{ }^{+}\left([\mathrm{M}]^{+}\right) 361.1241$; found: 361.1242 .

$[\alpha]_{\mathrm{D}}^{21}=-36.5\left(c=0.86, \mathrm{CHCl}_{3}\right)$. 


\section{Synthesis of $(S)$-1-Azido-2-[3-(R)-(N,N-dimethylamino)ethyl]ferrocen (2h)}

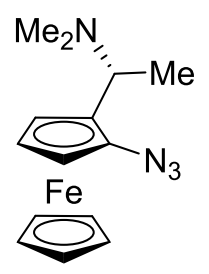

According to GP2, the title compound was prepared from $\mathbf{1 h}(0.10 \mathrm{~g}, 0.26 \mathrm{mmol}, 1.0$ equiv), $n$ butyllithium ( $0.12 \mathrm{~mL}, 0.29 \mathrm{mmol}, 1.1$ equiv.; $2.4 \mathrm{M}$ in $n$-hexane), and tosyl azide ( $0.42 \mathrm{~mL}, 0.31 \mathrm{mmol}$, 1.2 equiv.; $0.75 \mathrm{M}$ in THF) in anhydrous THF ( $3 \mathrm{~mL}$ ). Gravity column chromatography (basic $\mathrm{Al}_{2} \mathrm{O}_{3}$, activity grade $\mathrm{I}+5 \mathrm{wt} \% \mathrm{H}_{2} \mathrm{O} ; \mathrm{CH}_{2} \mathrm{Cl}_{2} / \mathrm{EtOAc} 100: 0$ to $5: 1$ ) gave the title compound $\mathbf{2 h}$ (55 mg, $0.19 \mathrm{mmol}, 71 \%$; d.r. $\left.=96: 4\left[{ }^{1} \mathrm{H} \mathrm{NMR}\right]\right)$ as a brown oil.

$\mathrm{R}_{\mathrm{f}}=0.50\left(\mathrm{SiO}_{2}, \mathrm{CH}_{2} \mathrm{Cl}_{2} / \mathrm{MeOH} 9: 1\right.$, vis. vanillin $)$.

${ }^{1} \mathrm{H} \mathrm{NMR}\left(600 \mathrm{MHz}, \mathrm{CDCl}_{3}\right): \delta[\mathrm{ppm}]=4.36(\mathrm{dd}, J=2.6,1.4 \mathrm{~Hz}, 1 \mathrm{H}), 4.23(\mathrm{~s}, 5 \mathrm{H}), 4.04(\mathrm{t}, J=2.6 \mathrm{~Hz}$, $1 \mathrm{H}), 4.02(\mathrm{dd}, J=2.7,1.4 \mathrm{~Hz}, 1 \mathrm{H}), 3.76(\mathrm{q}, J=6.9 \mathrm{~Hz}, 1 \mathrm{H}), 2.10(\mathrm{~s}, 6 \mathrm{H}), 1.44(\mathrm{~d}, J=6.9 \mathrm{~Hz}, 3 \mathrm{H})$.

${ }^{13} \mathrm{C}\left\{{ }^{1} \mathrm{H}\right\}$ NMR $\left(151 \mathrm{MHz}, \mathrm{CDCl}_{3}\right): \delta[\mathrm{ppm}]=98.0,81.8,69.8,65.2,63.3,59.5,55.3,40.8,15.1$.

HRMS (ESI): $\mathrm{m} / \mathrm{z}$ calcd. for $\mathrm{C}_{14} \mathrm{H}_{19} \mathrm{FeN}_{4}{ }^{+}\left([\mathrm{M}+\mathrm{H}]^{+}\right)$299.0954; found: 299.0965 .

The spectroscopic data are consistent with those reported in the literature. ${ }^{[1]}$

\section{Synthesis of 1-azido-1'-(trimethylsilylethynyl)ferrocene (2i)}

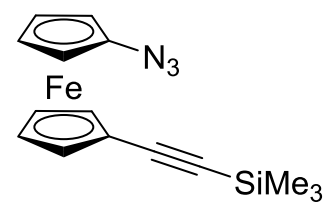

According to GP2, the title compound was prepared from 1i (100 mg, $0.245 \mathrm{mmol}, 1.0$ equiv), $n$ butyllithium ( $99.8 \mu \mathrm{L}, 0.270 \mathrm{mmol}, 1.1$ equiv; $2.7 \mathrm{M}$ in $n$-hexane), and tosyl azide $(118 \mu \mathrm{L}$, $0.294 \mathrm{mmol}, 1.2$ equiv; $2.5 \mathrm{M}$ in THF) in anhydrous THF ( $3 \mathrm{~mL})$. Gravity column chromatography (basic $\mathrm{Al}_{2} \mathrm{O}_{3}$, activity grade $\mathrm{I}+5 \mathrm{wt} \% \mathrm{H}_{2} \mathrm{O}$; $n$-pentane) gave the title compound $\mathbf{2 i}$ ( $63.0 \mathrm{mg}, 0.195 \mathrm{mmol}$, $79 \%)$ as an orange oil.

$\mathrm{R}_{\mathrm{f}}=0.57\left(\mathrm{SiO}_{2} ; n\right.$-pentane/ $\mathrm{CH}_{2} \mathrm{Cl}_{2}$ 9:1; vis. vanillin $)$.

${ }^{1} \mathrm{H} \mathrm{NMR}\left(400 \mathrm{MHz}, \mathrm{CDCl}_{3}\right) \delta[\mathrm{ppm}]=4.54(\mathrm{t}, J=1.9 \mathrm{~Hz}, 2 \mathrm{H}), 4.26(\mathrm{dt}, J=3.3,1.9 \mathrm{~Hz}, 4 \mathrm{H}), 4.07(\mathrm{t}$, $J=2.0 \mathrm{~Hz}, 2 \mathrm{H}), 0.22(\mathrm{~s}, 9 \mathrm{H})$.

${ }^{13} \mathrm{C}\left\{{ }^{1} \mathrm{H}\right\}$ NMR $\left(100 \mathrm{MHz}, \mathrm{CDCl}_{3}\right) \delta[\mathrm{ppm}]=103.0,100.3,92.0,73.0,69.8,66.8,67.4,62.3,0.21$.

FT-IR (neat) $\tilde{v}\left[\mathrm{~cm}^{-1}\right]=2958(\mathrm{w}), 2148(\mathrm{~m}), 2109$ (s), $1459(\mathrm{~m}), 1369(\mathrm{w}), 1287(\mathrm{~m}), 1249(\mathrm{~m}), 1163$ (w), $1026(\mathrm{w}), 927(\mathrm{~m}), 842(\mathrm{~s}), 759(\mathrm{~m}), 741(\mathrm{w}), 726(\mathrm{w}), 699(\mathrm{w})$.

HRMS (ESI-TOF) m/z calcd. for $\mathrm{C}_{15} \mathrm{H}_{18} \mathrm{FeN}_{3} \mathrm{Si}^{+}\left([\mathrm{M}+\mathrm{H}]^{+}\right)$324.0614; found: 324.0601 . 


\section{Synthesis of 1-azido-1'-(ethynylcyclopropyl)ferrocene (2j)}

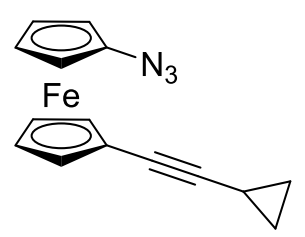

According to GP2, the title compound was prepared from $\mathbf{1 j}$ ( $76 \mathrm{mg}, 0.20 \mathrm{mmol}, 1.0$ equiv), $n$ butyllithium ( $90 \mu \mathrm{L}, 0.22 \mathrm{mmol}, 1.1$ equiv; $2.4 \mathrm{M}$ in $n$-hexane), and tosyl azide ( $0.33 \mathrm{~mL}, 0.24 \mathrm{mmol}$, 1.2 equiv; $0.75 \mathrm{M}$ in THF) in THF ( $2.5 \mathrm{~mL}$ ). Gravity column chromatography (basic $\mathrm{Al}_{2} \mathrm{O}_{3}$, activity grade $\mathrm{I}+5 \mathrm{wt} \% \mathrm{H}_{2} \mathrm{O} ; n$-pentane) gave the title compound $2 \mathbf{j}(33 \mathrm{mg}, 0.11 \mathrm{mmol}, 56 \%$ ) as a brown oil. $R_{\mathrm{f}}=0.23\left(\mathrm{SiO}_{2} ; n\right.$-pentane $/ \mathrm{CH}_{2} \mathrm{Cl}_{2} 10: 1 ;$ vis. vanillin $)$.

${ }^{1} \mathrm{H}$ NMR $\left(700 \mathrm{MHz}, \mathrm{CDCl}_{3}\right) \delta[\mathrm{ppm}]=4.45(\mathrm{~s}, 2 \mathrm{H}), 4.24(\mathrm{~s}, 2 \mathrm{H}), 4.21(\mathrm{~s}, 2 \mathrm{H}), 4.05(\mathrm{~s}, 2 \mathrm{H}), 1.34(\mathrm{tt}$, $J=8.3,4.8 \mathrm{~Hz}, 1 \mathrm{H}), 0.81(\mathrm{dq}, J=7.2,4.0 \mathrm{~Hz}, 2 \mathrm{H}), 0.76(\mathrm{dq}, J=7.6,4.9,4.3 \mathrm{~Hz}, 2 \mathrm{H})$.

${ }^{13} \mathrm{C}\left\{{ }^{1} \mathrm{H}\right\}$ NMR $\left(176 \mathrm{MHz}, \mathrm{CDCl}_{3}\right) \delta[\mathrm{ppm}]=99.9,90.7,72.5,69.3,68.4,67.1,62.0,8.5,0.5$. One resonance is missing presumably due to solvent overlap.

FT-IR (neat) $\tilde{v}\left[\mathrm{~cm}^{-1}\right]=3091(\mathrm{w}), 3009(\mathrm{w}), 2923(\mathrm{w}), 2852(\mathrm{w}), 2442(\mathrm{w}), 2321(\mathrm{w}), 2201(\mathrm{w}), 2105$ (s), 2204 (m), 1457 (s), 1370 (m), 1285 (s), 1260 (m), 1223 (w), 1204 (w), 1162 (m), 1116 (w), 1089 (w), 1051 (m), 1024 (s), $966(\mathrm{w}), 918(\mathrm{~m}), 897$ (s), 808 (s), 768 (w), 741 (m).

HRMS (ESI-TOF) m/z calcd. for $\mathrm{C}_{15} \mathrm{H}_{13} \mathrm{FeN}_{3} \mathrm{H}^{+}\left(\left[\mathrm{M}+\mathrm{H}^{+}\right]\right)$292.0532; found: 292.0544 .

\section{Synthesis of 1-azido-1'-phenylferrocene (2k)}

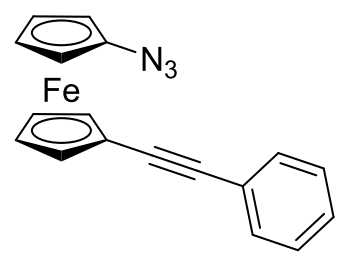

According to GP2, the title compound was prepared from 1k (41 mg, $0.10 \mathrm{mmol}, 1.0$ equiv), $n$ butyllithium ( $44 \mu \mathrm{L}, 0.11 \mathrm{mmol}, 1.1$ equiv; $2.5 \mathrm{M}$ in $n$-hexane), and tosyl azide ( $48 \mu \mathrm{L}, 0.12 \mathrm{mmol}$, 1.2 equiv; $2.5 \mathrm{M}$ in THF) in THF ( $2 \mathrm{~mL}$ ). Gravity column chromatography (basic $\mathrm{Al}_{2} \mathrm{O}_{3}$, activity grade $\mathrm{I}+5 \mathrm{wt} \% \mathrm{H}_{2} \mathrm{O}, n$-pentane) gave the title compound $2 \mathbf{k}(31 \mathrm{mg}, 0.095 \mathrm{mmol}, 95 \%)$ as an orange solid. $R_{\mathrm{f}}=0.38\left(\mathrm{SiO}_{2} ; n\right.$-pentane $/ \mathrm{CH}_{2} \mathrm{Cl}_{2}$ 9:1, vis. vanillin $)$.

${ }^{1} \mathrm{H}$ NMR $\left(400 \mathrm{MHz}, \mathrm{CDCl}_{3}\right) \delta[\mathrm{ppm}]=7.52-7.48(\mathrm{~m}, 2 \mathrm{H}), 7.36-7.29(\mathrm{~m}, 3 \mathrm{H}), 4.62(\mathrm{t}, J=1.9 \mathrm{~Hz}$, $2 \mathrm{H}), 4.33(\mathrm{t}, J=1.9 \mathrm{~Hz}, 2 \mathrm{H}), 4.31(\mathrm{t}, J=2.0 \mathrm{~Hz}, 2 \mathrm{H}), 4.12(\mathrm{t}, J=2.0 \mathrm{~Hz}, 2 \mathrm{H})$.

${ }^{13} \mathrm{C}\left\{{ }^{1} \mathrm{H}\right\} \mathrm{NMR}\left(100 \mathrm{MHz}, \mathrm{CDCl}_{3}\right) \delta[\mathrm{ppm}]=131.6,128.4,127.9,123.9,100.2,87.2,86.9,77.4,72.7$, $69.9,67.2,62.2$. 
FT-IR (neat) $\tilde{v}\left[\mathrm{~cm}^{-1}\right]=3084(\mathrm{w}), 3057(\mathrm{w}), 2442$ (w), 2321 (w), 2206 (w), 2106 (s), 2024 (w), 1599 (w), 1571 (w), 1497 (m), 1458 (s), 1442 (m), 1369 (w), 1285 (m), 1223 (w), 1204 (w), 1162 (m), 1070 (w), $1026(\mathrm{~m}), 924(\mathrm{~m}), 916(\mathrm{~m}), 812(\mathrm{~m}), 755$ (s), $740(\mathrm{~m}), 690(\mathrm{~m})$.

HRMS (ESI-TOF) m/z calcd. for $\mathrm{C}_{18} \mathrm{H}_{13} \mathrm{FeN}_{3}{ }^{+}\left([\mathrm{M}]^{+}\right)$328.0532; found: 328.0535 .

\section{Synthesis of 1-azido-1'-(1-ethynyl-4-methoxybenzene)ferrocene (2l)}

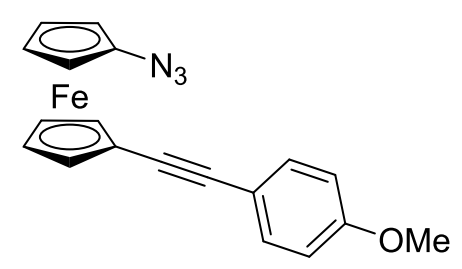

According to GP2, the title compound was prepared from 11 ( $87 \mathrm{mg}, 0.20 \mathrm{mmol}, 1$ equiv), $n$ butyllithium ( $0.18 \mathrm{~mL}, 0.22 \mathrm{mmol}, 1.1$ equiv; $1.2 \mathrm{M}$ in $n$-hexane), and tosyl azide ( $0.32 \mathrm{~mL}, 0.24 \mathrm{mmol}$, 1.2 equiv; $0.75 \mathrm{M}$ in THF) in THF ( $2 \mathrm{~mL}$ ). Gravity column chromatography (basic $\mathrm{Al}_{2} \mathrm{O}_{3}$, activity grade $\mathrm{I}+5 \mathrm{wt} \% \mathrm{H}_{2} \mathrm{O} ; n$-pentane $/ \mathrm{CH}_{2} \mathrm{Cl}_{2} 5: 1$ ) followed by recrystallization from $n$-hexane at $-78{ }^{\circ} \mathrm{C}$ gave the title compound $2 \mathrm{l}(47 \mathrm{mg}, 0.13 \mathrm{mmol}, 67 \%)$ as an orange solid.

$R_{\mathrm{f}}=0.36\left(\mathrm{SiO}_{2}, n\right.$-pentane $/ \mathrm{CH}_{2} \mathrm{Cl}_{2} 4: 1$; vis. vanillin $)$.

${ }^{1} \mathrm{H}$ NMR $\left(700 \mathrm{MHz}, \mathrm{CDCl}_{3}\right) \delta[\mathrm{ppm}]=7.43(\mathrm{~d}, J=8.8 \mathrm{~Hz}, 2 \mathrm{H}), 6.86(\mathrm{~d}, J=8.8 \mathrm{~Hz}, 2 \mathrm{H}), 4.59(\mathrm{t}$, $J=1.9 \mathrm{~Hz}, 2 \mathrm{H}), 4.31(\mathrm{t}, J=1.8 \mathrm{~Hz}, 2 \mathrm{H}), 4.31-4.30(\mathrm{~m}, 2 \mathrm{H}), 4.11(\mathrm{t}, J=2.0 \mathrm{~Hz}, 2 \mathrm{H}), 3.82(\mathrm{~s}, 3 \mathrm{H})$.

${ }^{13} \mathrm{C}\left\{{ }^{1} \mathrm{H}\right\} \operatorname{NMR}\left(176 \mathrm{MHz}, \mathrm{CDCl}_{3}\right) \delta[\mathrm{ppm}]=159.4,133.0,116.0,114.1,100.2,86.8,85.5,72.5,69.7$, $67.8,67.2,62.1,55.4$.

FT-IR (neat) $\tilde{v}\left[\mathrm{~cm}^{-1}\right]=3090(\mathrm{w}), 3001(\mathrm{w}), 2920(\mathrm{w}), 2837(\mathrm{w}), 2441(\mathrm{w}), 2360(\mathrm{~m}), 2341(\mathrm{~m}), 2203$ (w), 2108 (s), 2205 (w), 1718 (w), 1604 (m), 1568 (w), 1513 (m), 1458 (m), 1441 (w), 1370 (w), 1284 (m), 1246 (s), 1164 (m), 1106 (w), 1027 (m), 925 (w), 830 (m), 813 (m), 733 (m), 668 (m).

HRMS (ESI-TOF) m/z calcd. for $\mathrm{C}_{19} \mathrm{H}_{15} \mathrm{FeN}_{3} \mathrm{ONa}^{+}$([M+Na] $]^{+}$380.0457; found: 380.0441 .

\section{Synthesis of 1-azido-1'-(1-ethynyl-4-trifluoromethylbenzene)ferrocene (2m)}

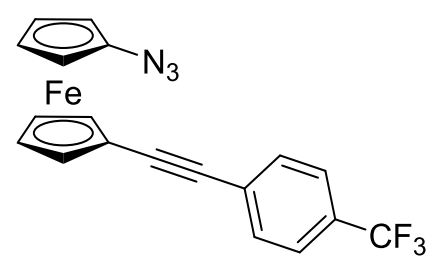

According to GP2, the title compound was prepared from $\mathbf{1 m}$ (47 mg, $98 \mu \mathrm{mol}, 1.0$ equiv), $n$ butyllithium ( $40 \mu \mathrm{L}, 0.11 \mathrm{mmol}, 1.1$ equiv; $2.4 \mathrm{M}$ in $n$-hexane), and tosyl azide $(0.16 \mathrm{~mL}, 0.12 \mathrm{mmol}$, 1.2 equiv; $0.75 \mathrm{M}$ in THF) in THF $(1.5 \mathrm{~mL})$. Gravity column chromatography (basic $\mathrm{Al}_{2} \mathrm{O}_{3}$, activity grade $\mathrm{I}+5 \mathrm{wt} \% \mathrm{H}_{2} \mathrm{O}, n$-pentane) gave the title compound $\mathbf{2 m}(29 \mathrm{mg}, 74 \mu \mathrm{mol}, 75 \%)$ as an orange solid. 
$R_{\mathrm{f}}=0.26\left(\mathrm{SiO}_{2} ; n\right.$-pentane, vis. vanillin $)$.

${ }^{1} \mathrm{H} \mathrm{NMR}\left(700 \mathrm{MHz}, \mathrm{CDCl}_{3}\right) \delta[\mathrm{ppm}]=7.58(\mathrm{~s}, 4 \mathrm{H}), 4.64(\mathrm{t}, J=1.8 \mathrm{~Hz}, 2 \mathrm{H}), 4.37(\mathrm{t}, J=1.8 \mathrm{~Hz}, 2 \mathrm{H})$, $4.32(\mathrm{t}, J=1.9 \mathrm{~Hz}, 2 \mathrm{H}), 4.13(\mathrm{t}, J=1.9 \mathrm{~Hz}, 2 \mathrm{H})$.

${ }^{13} \mathrm{C}\left\{{ }^{1} \mathrm{H}\right\} \operatorname{NMR}\left(176 \mathrm{MHz}, \mathrm{CDCl}_{3}\right) \delta[\mathrm{ppm}]=131.7,129.5(\mathrm{q}, J=32.4 \mathrm{~Hz}), 127.7,125.4(\mathrm{~d}, J=3.7 \mathrm{~Hz})$, $124.2(\mathrm{q}, J=272.2 \mathrm{~Hz}), 100.3,90.2,85.7,72.9,70.3,67.3,66.4,62.2$.

${ }^{19} \mathrm{~F} \mathrm{NMR}\left(565 \mathrm{MHz}, \mathrm{CDCl}_{3}\right) \delta[\mathrm{ppm}]=-62.6$.

FT-IR (neat): $\tilde{v}\left[\mathrm{~cm}^{-1}\right]=2961(\mathrm{w}), 2923(\mathrm{w}), 2361(\mathrm{w}), 2205$ (w), $2110(\mathrm{~s}), 1607(\mathrm{~m}), 1460(\mathrm{~m}), 1406$ (m), 1371 (w), 1321 (s), 1288 (m), 1261 (m), 1161 (s), 1117 (s), 1103 (s), 1065 (s), 1029 (s), 1016 (s), $918(\mathrm{~m}), 838(\mathrm{~s}), 802(\mathrm{~s}), 745(\mathrm{~m})$.

HRMS (ESI-TOF) m/z calcd. for $\mathrm{C}_{19} \mathrm{H}_{12} \mathrm{~F}_{3} \mathrm{FeN}_{3}{ }^{+}\left([\mathrm{M}]^{+}\right)$395.0333; found: 395.0321 .

\section{Synthesis of 1-azido-1'-(ethynylpyridyl)ferrocene (2n)}

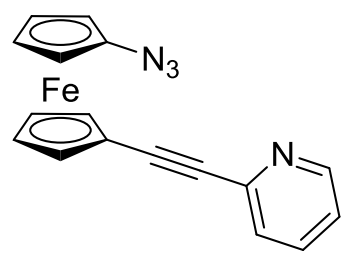

According to GP2, the title compound was prepared from 1n (70 mg, $0.17 \mathrm{mmol}, 1.0$ equiv), $n$ butyllithium ( $75 \mu \mathrm{L}, 0.19 \mathrm{mmol}, 1.1$ equiv; $2.4 \mathrm{M}$ in $n$-hexane), and tosyl azide $(0.27 \mathrm{~mL}, 0.20 \mathrm{mmol}$, 1.2 equiv; $0.75 \mathrm{M}$ in THF) in THF (3.5 mL). Gravity column chromatography (basic $\mathrm{Al}_{2} \mathrm{O}_{3}$, activity grade $\mathrm{I}+5 \mathrm{wt} \% \mathrm{H}_{2} \mathrm{O} ; n$-hexane/EtOAc 10:1) gave the title compound $2 \mathrm{n}$ (42 $\mathrm{mg}, 0.13 \mathrm{mmol}, 76 \%$ ) without further purification as a brown oil.

$R_{\mathrm{f}}=0.27\left(\mathrm{SiO}_{2} ; n\right.$-hexane/EtOAc 4:1; vis. vanillin $)$

${ }^{1} \mathrm{H} \mathrm{NMR}\left(700 \mathrm{MHz}, \mathrm{CDCl}_{3}\right) \delta[\mathrm{ppm}]=8.58(\mathrm{dt}, J=4.7,1.5 \mathrm{~Hz}, 1 \mathrm{H}), 7.64(\mathrm{td}, J=7.7,1.8 \mathrm{~Hz}, 1 \mathrm{H}), 7.46$ (dt, $J=7.8,1.1 \mathrm{~Hz}, 1 \mathrm{H}), 7.21(\mathrm{ddd}, J=7.6,4.9,1.2 \mathrm{~Hz}, 1 \mathrm{H}), 4.68(\mathrm{t}, J=1.9 \mathrm{~Hz}, 2 \mathrm{H}), 4.37$ (t, $J=1.9 \mathrm{~Hz}$, $2 \mathrm{H}), 4.32(\mathrm{t}, J=2.0 \mathrm{~Hz}, 2 \mathrm{H}), 4.13(\mathrm{t}, J=2.0,2 \mathrm{H})$.

${ }^{13} \mathrm{C}\left\{{ }^{1} \mathrm{H}\right\}$ NMR $\left(176 \mathrm{MHz}, \mathrm{CDCl}_{3}\right) \delta[\mathrm{ppm}]=150.1,144.0,136.2,126.9,122.4,100.4,88.1,86.4,73.1$, $70.4,67.6,65.7,62.2$.

FT-IR (neat) $\tilde{v}\left[\mathrm{~cm}^{-1}\right]=3078(\mathrm{w}), 2920(\mathrm{w}), 2849(\mathrm{w}), 2454(\mathrm{w}), 2206(\mathrm{~m}), 2106(\mathrm{~s}), 2027(\mathrm{~m}), 1578$ (s), 1560 (m), 1477 (m), 1460 (s), 1423 (m), 1366 (w), 1292 (s), 1227 (w), 1174 (w), 1164 (w), 1153 (w), 1091 (w), 1024 (s), 987 (m), 918 (m), 807 (s), 772 (s), 736 (s).

HRMS (ESI-TOF) m/z calcd. for $\mathrm{C}_{17} \mathrm{H}_{12} \mathrm{FeN}_{4} \mathrm{~K}^{+}\left([\mathrm{M}+\mathrm{K}]^{+}\right)$367.0043; found: 367.0061 . 


\subsection{Synthesis of Ferrocenyl Azides in Flow}

\section{Preparation of a stock solution of $\boldsymbol{n}$-butyllithium in $\boldsymbol{n}$-hexane}

In a dry Schlenk tube, a stock solution of $n$-butyllithium in $n$-hexane with a concentration of $1.2 \mathrm{M}$ was prepared. This solution was stored in the dark at $8{ }^{\circ} \mathrm{C}$ and could be used for several months without changes in yield. However, it is recommended to titrate this stock solution from time to time.

\section{General Procedure 3 (GP3) for the synthesis of ferrocenyl azides in flow}

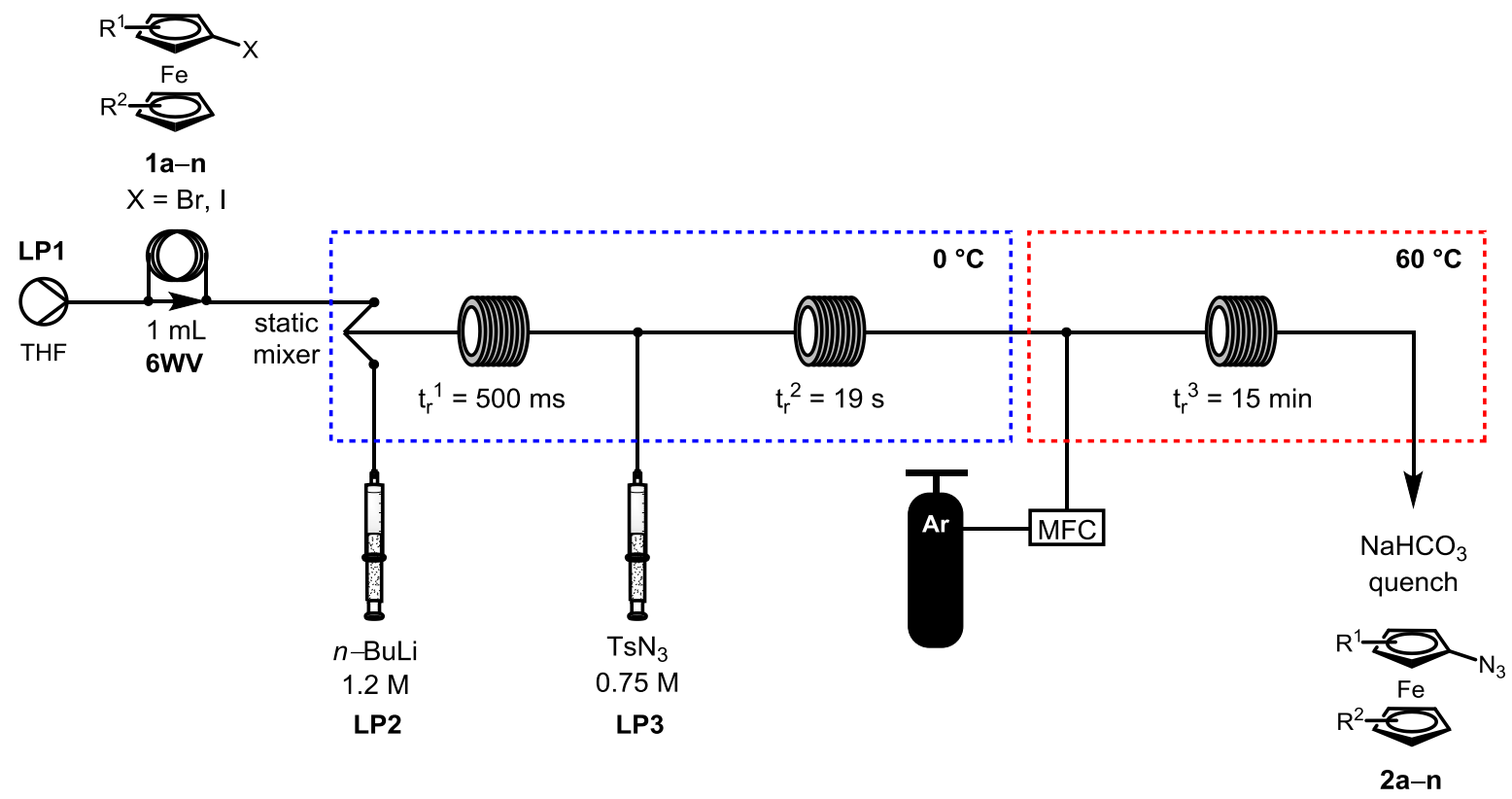

Syringe pumps (LP2, LP3) equipped with gas-tight $10 \mathrm{~mL}$ glass-syringes were used to pump solutions of $n$-butyllithium (1.2 M $n$-hexane) and tosyl azide ( $0.75 \mathrm{M}$ in THF). Both solutions were passed through precooling loops (FEP tube, outer diameter 1/16", inner diameter 1/32", V $=0.15 \mathrm{~mL}$ ) before mixing. A HPLC pump (LP1) to feed the solvent was connected to a HPLC injection valve (6WV) equipped with a $1 \mathrm{~mL}$ sample loop. The liquid supply was passed through a precooled loop (FEP tube, outer diameter 1/16", inner diameter $1 / 32 ", \mathrm{~V}=0.15 \mathrm{~mL}$ ) and then merged in a static mixer (internal volume: $20 \mu \mathrm{L}$ ) with a solution of $n$-butyllithium (1.2 M $n$-hexane). The resulting reaction stream was pumped through tube reactor1 (stainless steel capillary, inner diameter $250 \mu \mathrm{m}, \mathrm{V}=20 \mu \mathrm{L}$ ) and then merged in a stainless steel T-mixer with a solution of tosyl azide. Reactor1, reactor2, the mixing units, and the precooled loops were cooled to $0{ }^{\circ} \mathrm{C}$ by immersion into an ice bath.

The reaction stream was mixed in a T-mixer with argon provided by a mass flow controller (MFC) resulting in a segmented flow pattern. This stream was pumped through tube reactor3 (PTFE tube, inner diameter $1 / 8 ", \mathrm{~V}=40 \mathrm{~mL}$ ) heated to $60^{\circ} \mathrm{C}$ in a water bath. The resulting triphasic flow regime was collected in a flask containing $\mathrm{NaHCO}_{3}$ (sat. aq., $50 \mathrm{~mL}$ ). 
The HPLC pump and the reactor were washed with anhydrous THF for 2 residence times by pumping THF with the HPLC pump and feeding argon using the MFC ( $\mathrm{v}_{\text {argon }}=0.2 \mathrm{~mL} / \mathrm{min}$ ) until a steady state was achieved. A solution of the ferrocenyl halide (1a-1n, $0.2 \mathrm{M}$ in THF) was loaded on the HPLC injection valve. The solutions of $n$-butyllithium $\left(\mathrm{v}_{n-\mathrm{BuLi}}=0.4 \mathrm{~mL} / \mathrm{min}\right)$ and tosyl azide $\left(\mathrm{v}_{\mathrm{TsN} 3}=0.8 \mathrm{~mL} / \mathrm{min}\right)$ were pumped at the given flow rate for $15-20 \mathrm{~s}$. Then, the solution of the ferrocenyl halide $(\mathbf{1 a}-\mathbf{1 n})$ was injected $\left(\mathrm{v}_{\mathrm{FcX}}=2.0 \mathrm{~mL} / \mathrm{min}\right)$ into the system by switching the HPLC valve to the "inject" position. The collected reaction mixture was diluted with EtOAc $(50 \mathrm{~mL})$. The aqueous layer was separated and extracted with EtOAc $(2 \times 50 \mathrm{~mL})$. The combined organic layers were dried $\left(\mathrm{Na}_{2} \mathrm{SO}_{4}\right)$, filtered, and concentrated under reduced pressure. The corresponding ferrocenyl azide (2a-2n) was isolated by gravity column chromatography.

Note 1: Drying with $\mathrm{MgSO}_{4}$ instead of $\mathrm{Na}_{2} \mathrm{SO}_{4}$ gave lower yields.

Note 2: For column chromatography, the crude product was suspended in a small amount of the eluent and the resulting suspension was directly loaded onto the column using a Pasteur pipette.

Note 3: In some cases, flash column chromatography was possible, but in general, gravity column chromatography gave better separations.

\section{Synthesis of azidoferrocene (2a)}

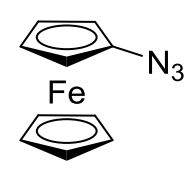

According to GP3, the title compound was prepared from 1a (62 mg, $0.20 \mathrm{mmol})$. Gravity column chromatography (basic $\mathrm{Al}_{2} \mathrm{O}_{3}$, activity grade $\mathrm{I}+5 \mathrm{wt} \% \mathrm{H}_{2} \mathrm{O}$; $n$-pentane) gave the title compound $\mathbf{2 a}$ (37 mg, $0.16 \mathrm{mmol}, 82 \%$ ) as a red oil that solidified upon standing at $-18{ }^{\circ} \mathrm{C}$.

$R_{\mathrm{f}}=0.41\left(\mathrm{SiO}_{2}, n\right.$-hexane, vis. vanillin).

${ }^{1} \mathrm{H} \mathrm{NMR}\left(700 \mathrm{MHz}, \mathrm{CDCl}_{3}\right) \delta[\mathrm{ppm}]=4.28(\mathrm{~s}, 5 \mathrm{H}), 4.26(\mathrm{t}, J=1.9 \mathrm{~Hz}, 2 \mathrm{H}), 4.04(\mathrm{t}, J=1.9 \mathrm{~Hz}, 2 \mathrm{H})$.

${ }^{13} \mathrm{C}\left\{{ }^{1} \mathrm{H}\right\} \mathrm{NMR}\left(176 \mathrm{MHz}, \mathrm{CDCl}_{3}\right) \delta[\mathrm{ppm}]=99.3,69.3,65.5,60.7$. 


\section{Synthesis of azidoferrocene (2a) on gram scale}

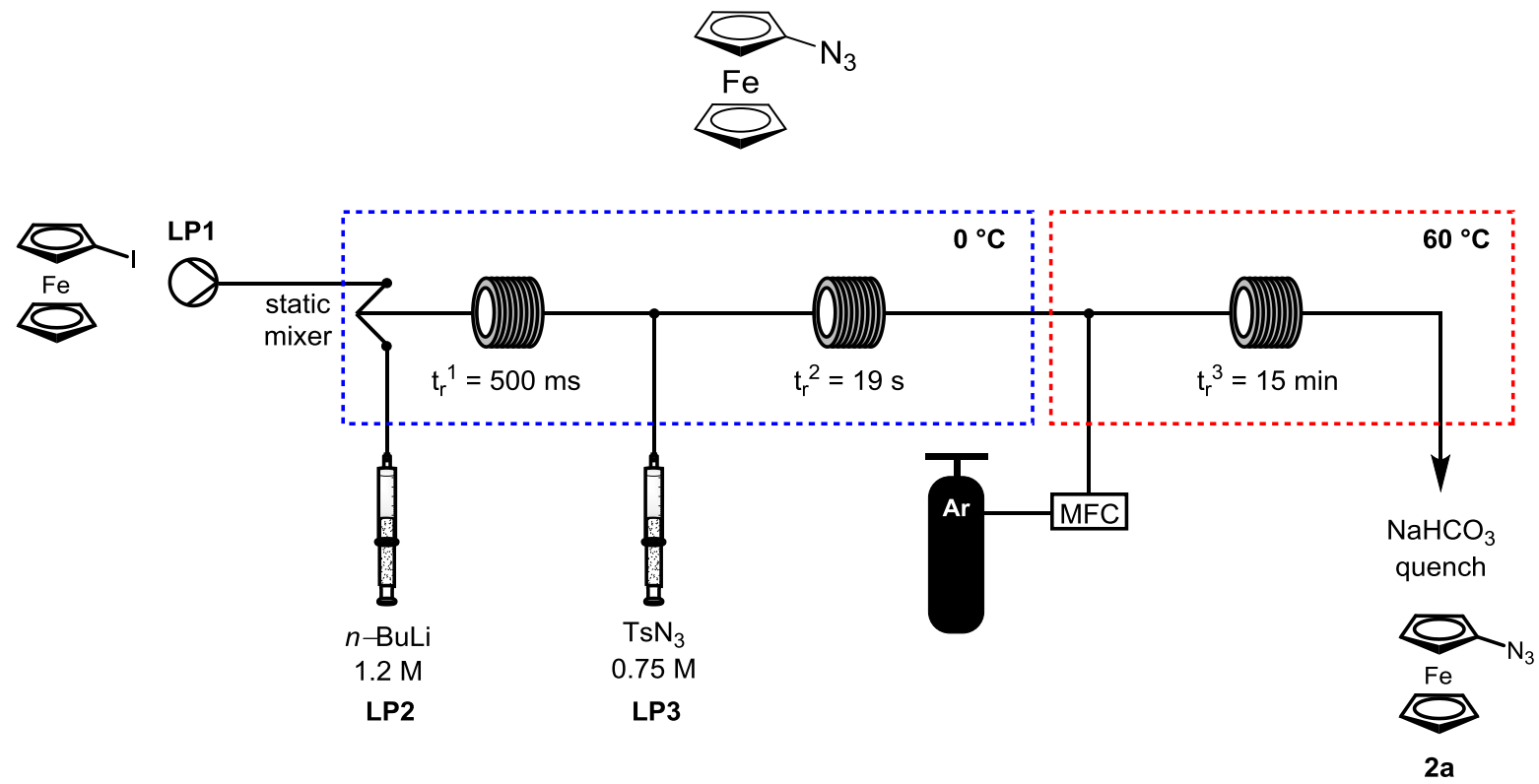

Syringe pumps (LP2, LP3) equipped with gas-tight $10 \mathrm{~mL}$ glass-syringes were used to pump solutions of $n$-butyllithium (1.2 $\mathrm{M} n$-hexane) and tosyl azide ( $0.75 \mathrm{M}$ in THF). Both solutions were passed through precooling loops (FEP tube, outer diameter 1/16", inner diameter 1/32", $\mathrm{V}=0.15 \mathrm{~mL}$ ) before mixing. A solution of iodoferrocene 1a ( $0.2 \mathrm{M}$ in THF) was provided by a HPLC pump (LP1) and was passed through a precooled loop (FEP tube, outer diameter 1/16", inner diameter $1 / 32$ ", $\mathrm{V}=0.15 \mathrm{~mL}$ ) and then merged in a static mixer (internal volume: $20 \mu \mathrm{L}$ ) with a solution of $n$-butyllithium (1.2 M $n$-hexane). The resulting reaction stream was pumped through tube reactor1 (stainless steel capillary, inner diameter $250 \mu \mathrm{m}, \mathrm{V}=20 \mu \mathrm{L}$ ) and then merged in a stainless steel T-mixer with a solution of tosyl azide.

Reactor1, reactor2, the mixing units, and the precooled loops were cooled to $0{ }^{\circ} \mathrm{C}$ by immersion into an ice bath.

The reaction stream was mixed in a T-mixer with argon provided by a mass flow controller (MFC) resulting in a segmented flow pattern. This stream was pumped through tube reactor3 (PTFE tube, inner diameter $1 / 8 ", V=40 \mathrm{~mL}$ ) heated to $60^{\circ} \mathrm{C}$ in a water bath. The resulting triphasic flow regime was collected in a flask containing $\mathrm{NaHCO}_{3}$ (sat. aq., $50 \mathrm{~mL}$ ).

The HPLC pump and the reactor were washed with anhydrous THF for 2 residence times by pumping THF with the HPLC pump and feeding argon using the MFC ( $\mathrm{v}_{\text {argon }}=0.2 \mathrm{~mL} / \mathrm{min}$ ) until a steady state was achieved. The solutions of $n$-butyllithium $\left(\mathrm{v}_{n \text {-BuLi }}=0.4 \mathrm{~mL} / \mathrm{min}\right)$ and tosyl azide $\left(\mathrm{v}_{\mathrm{TsN} 3}=0.8 \mathrm{~mL} / \mathrm{min}\right)$ were pumped at the given flow rate for $15-20 \mathrm{~s}$. Then, a solution of iodoferrocene 1a was pumped by the HPLC pump into the system. After a steady state has been reached, the reaction mixture was collected for $15 \mathrm{~min}$. Then, the reaction mixture was diluted with EtOAc $(50 \mathrm{~mL})$. The aqueous layer was separated and extracted with EtOAc $(2 \times 50 \mathrm{~mL})$. The combined organic layers were dried $\left(\mathrm{Na}_{2} \mathrm{SO}_{4}\right)$, filtered, and concentrated under reduced pressure. Gravity column chromatography 
(basic $\mathrm{Al}_{2} \mathrm{O}_{3}$, activity grade $\mathrm{I}+5 \mathrm{wt} \% \mathrm{H}_{2} \mathrm{O} ; n$-pentane) gave the title compound $2 \mathrm{a}$ (37 $\mathrm{mg}, 0.16 \mathrm{mmol}$, $82 \%$ ) as a red oil that solidified upon standing at $-18^{\circ} \mathrm{C}$. Gravity column chromatography (basic $\mathrm{Al}_{2} \mathrm{O}_{3}$, activity grade $\mathrm{I}+5 \mathrm{wt} \% \mathrm{H}_{2} \mathrm{O} ; n$-pentane) gave the title compound $\mathbf{2 a}(0.96 \mathrm{~g}, 4.2 \mathrm{mmol}, 69 \%)$ as a red oil that solidified upon standing at $-18{ }^{\circ} \mathrm{C}$.

The spectroscopic data are consistent with those reported above.

Synthesis of 1-azido-1'-bromoferrocene (2b)

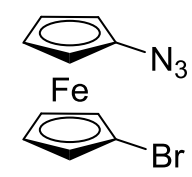

According to GP3, the title compound was prepared from $\mathbf{1 b}(83 \mathrm{mg}, 0.24 \mathrm{mmol})$. Gravity column chromatography (basic $\mathrm{Al}_{2} \mathrm{O}_{3}$, activity grade $\mathrm{I}+5 \mathrm{wt} \% \mathrm{H}_{2} \mathrm{O}$; $n$-pentane) gave the title compound $\mathbf{2 b}$ (59 $\mathrm{mg}, 0.19 \mathrm{mmol}, 80 \%)$ as a red oil that solidified upon standing.

$R_{\mathrm{f}}=0.32\left(\mathrm{SiO}_{2}, n\right.$-pentane, vis. vanillin).

${ }^{1} \mathrm{H} \mathrm{NMR}\left(700 \mathrm{MHz}, \mathrm{CDCl}_{3}\right) \delta[\mathrm{ppm}]=4.52(\mathrm{t}, J=1.9 \mathrm{~Hz}, 2 \mathrm{H}), 4.27(\mathrm{t}, J=1.9 \mathrm{~Hz}, 2 \mathrm{H}), 4.20(\mathrm{t}$, $J=1.9 \mathrm{~Hz}, 2 \mathrm{H}), 4.12(\mathrm{t}, J=1.9 \mathrm{~Hz}, 2 \mathrm{H})$.

${ }^{13} \mathrm{C}\left\{{ }^{1} \mathrm{H}\right\}$ NMR $\left(176 \mathrm{MHz}, \mathrm{CDCl}_{3}\right) \delta[\mathrm{ppm}]=100.7,78.7,71.5,68.3,67.9,62.8$.

\section{Synthesis of 1-azido-1'-iodoferrocene (2c)}

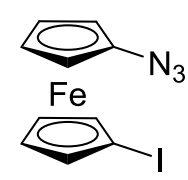

According to GP3, the title compound was prepared from 1c (105 mg, $0.240 \mathrm{mmol})$. Gravity column chromatography (basic $\mathrm{Al}_{2} \mathrm{O}_{3}$, activity grade $\mathrm{I}+5 \mathrm{wt} \% \mathrm{H}_{2} \mathrm{O}$; $n$-pentane) gave the title compound $\mathbf{2 c}$ (45.9 $\mathrm{mg}, 0.130 \mathrm{mmol}, 54 \%$ ) as a brown oil.

$R_{\mathrm{f}}=0.39\left(\mathrm{SiO}_{2}, n\right.$-pentane, vis. vanillin).

${ }^{1} \mathrm{H}$ NMR $\left(600 \mathrm{MHz}, \mathrm{CDCl}_{3}\right) \delta[\mathrm{ppm}]=4.51(\mathrm{t}, J=1.8 \mathrm{~Hz}, 2 \mathrm{H}), 4.25(\mathrm{t}, J=1.8 \mathrm{~Hz}, 2 \mathrm{H}), 4.22(\mathrm{t}$, $J=2.0 \mathrm{~Hz}, 2 \mathrm{H}), 4.08(\mathrm{t}, J=2.0 \mathrm{~Hz}, 2 \mathrm{H})$.

${ }^{13} \mathrm{C}\left\{{ }^{1} \mathrm{H}\right\}$ NMR $(151 \mathrm{MHz}, \mathrm{CDCl}) \delta[\mathrm{ppm}]=100.6,75.9,70.0,68.5,63.3,40.9$. 
Synthesis of 1,1'-diazidoferrocene (2d)

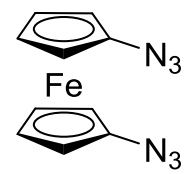

According to GP3, the title compound was prepared from $\mathbf{1 b}$ (34 $\mathrm{mg}, 0.10 \mathrm{mmol})$. Gravity column chromatography (basic $\mathrm{Al}_{2} \mathrm{O}_{3}$, activity grade $\mathrm{I}+5 \mathrm{wt} \% \mathrm{H}_{2} \mathrm{O} ; n$-pentane) gave the title compound $\mathbf{2 d}$ (20 mg, $0.075 \mathrm{mmol}, 75 \%$ ) as a brown oil.

$R_{\mathrm{f}}=0.29\left(\mathrm{SiO}_{2}, n\right.$-hexane, vis. vanillin).

${ }^{1} \mathrm{H}$ NMR $\left(600 \mathrm{MHz}, \mathrm{CDCl}_{3}\right) \delta[\mathrm{ppm}]=4.35(\mathrm{~s}, 4 \mathrm{H}), 4.15$ (s, 4H).

${ }^{13} \mathrm{C}\left\{{ }^{1} \mathrm{H}\right\}$ NMR $\left(151 \mathrm{MHz}, \mathrm{CDCl}_{3}\right) \delta[\mathrm{ppm}]=100.4,66.5,61.6$.

\section{Synthesis of 1-azido-1'-ferrocenylcarboxylic acid tert-butyl ester (2e)}

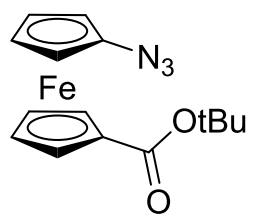

According to GP3, the title compound was prepared from 1e (73 mg, $0.20 \mathrm{mmol})$. Gravity column chromatography (basic $\mathrm{Al}_{2} \mathrm{O}_{3}$, activity grade $\mathrm{I}+5 \mathrm{wt} \% \mathrm{H}_{2} \mathrm{O} ; n$-pentane/ $\mathrm{Et}_{2} \mathrm{O}$ 20:1) gave the title compound $2 \mathbf{e}$ (47 mg, $0.14 \mathrm{mmol}, 71 \%$ ) as a red oil.

$R_{\mathrm{f}}=0.40\left(\mathrm{SiO}_{2}, n\right.$-pentane/ $\mathrm{Et}_{2} \mathrm{O}=20: 1$, vis. vanillin $)$.

${ }^{1} \mathrm{H}$ NMR $\left(700 \mathrm{MHz}, \mathrm{CDCl}_{3}\right) \delta[\mathrm{ppm}]=4.84(\mathrm{t}, J=1.9 \mathrm{~Hz}, 2 \mathrm{H}), 4.44(\mathrm{t}, J=1.9 \mathrm{~Hz}, 2 \mathrm{H}), 4.26(\mathrm{t}$, $J=1.9 \mathrm{~Hz}, 2 \mathrm{H}), 4.08(\mathrm{t}, J=1.9 \mathrm{~Hz}, 2 \mathrm{H}), 1.57(\mathrm{~s}, 9 \mathrm{H})$.

${ }^{13} \mathrm{C}\left\{{ }^{1} \mathrm{H}\right\} \mathrm{NMR}\left(176 \mathrm{MHz}, \mathrm{CDCl}_{3}\right) \delta[\mathrm{ppm}]=170.3,100.6,80.6,75.0,72.1,71.2,67.1,61.8,28.4$.

\section{Synthesis of 1-azido-1'-(1H-benzo[d][1,2,3]triazole)ferrocene (2f)}

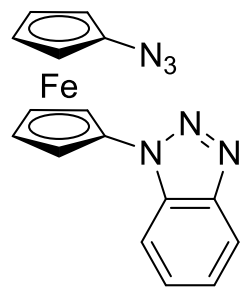

According to GP3, the title compound was prepared from 1f (76 mg, $0.20 \mathrm{mmol})$. Gravity column chromatography (basic $\mathrm{Al}_{2} \mathrm{O}_{3}$, activity grade $\mathrm{I}+5 \mathrm{wt} \% \mathrm{H}_{2} \mathrm{O}$; $n$-hexane/EtOAc 4:1) gave the title compound $\mathbf{2 f}$ (44 $\mathrm{mg}, 0.13 \mathrm{mmol}, 64 \%$ ) as a brown solid.

$R_{\mathrm{f}}=0.30\left(\mathrm{SiO}_{2}, n\right.$-hexane/EtOAc 4:1). 
${ }^{1} \mathrm{H}$ NMR $\left(600 \mathrm{MHz}, \mathrm{CDCl}_{3}\right) \delta[\mathrm{ppm}]=8.12(\mathrm{~d}, J=8.3 \mathrm{~Hz}, 1 \mathrm{H}), 7.82(\mathrm{~d}, J=8.4 \mathrm{~Hz}, 1 \mathrm{H}), 7.57(\mathrm{t}, J=$ $7.7 \mathrm{~Hz}, 1 \mathrm{H}), 7.42(\mathrm{t}, J=7.8 \mathrm{~Hz}, 1 \mathrm{H}), 5.08$ (s, 2H), 4.48 (s, 2H), 4.34 (s, 2H), 4.15 (s, 2H).

${ }^{13} \mathrm{C}\left\{{ }^{1} \mathrm{H}\right\} \mathrm{NMR}\left(151 \mathrm{MHz}, \mathrm{CDCl}_{3}\right) \delta[\mathrm{ppm}]=146.5,133.0,128.0,124.4,120.5,110.7,100.9,94.7,67.8$, 67.4, 63.6, 62.1.

\section{Synthesis of 1-azido-1'-[(1S, 4S)- 1,7,7-trimethylbicyclo[2.2.1]hept-2-enyl]ferrocene (2g)}

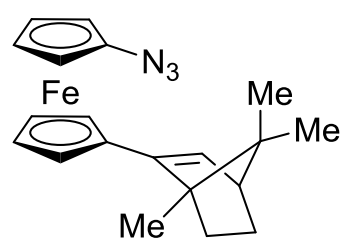

According to GP3, the title compound was prepared from $\mathbf{1 g}(80 \mathrm{mg}, 0.20 \mathrm{mmol})$. Gravity column chromatography (basic $\mathrm{Al}_{2} \mathrm{O}_{3}$, activity grade $\mathrm{I}+5 \mathrm{wt} \% \mathrm{H}_{2} \mathrm{O} ; n$-hexane) gave the title compound $\mathbf{2 g}$ (48 $\mathrm{mg}, 0.13 \mathrm{mmol}, 66 \%)$ as an orange oil.

$R_{\mathrm{f}}=0.42\left(\mathrm{SiO}_{2} ; n\right.$-pentane; vis. vanillin).

${ }^{1} \mathrm{H}$ NMR $\left(600 \mathrm{MHz}, \mathrm{CDCl}_{3}\right) \delta[\mathrm{ppm}]=6.04(\mathrm{~d}, J=3.3 \mathrm{~Hz}, 1 \mathrm{H}), 4.39(\mathrm{~d}, J=14.3 \mathrm{~Hz}, 2 \mathrm{H}), 4.31(\mathrm{~s}, 2 \mathrm{H})$, $4.18(\mathrm{~d}, J=12.4 \mathrm{~Hz}, 2 \mathrm{H}), 4.01(\mathrm{~s}, 2 \mathrm{H}), 2.30(\mathrm{t}, J=3.3 \mathrm{~Hz}, 1 \mathrm{H}), 1.88(\mathrm{td}, J=8.6,3.8 \mathrm{~Hz}, 1 \mathrm{H}), 1.58(\mathrm{ddd}$, $J=11.9,8.4,3.6 \mathrm{~Hz}, 1 \mathrm{H}), 1.24(\mathrm{~s}, 3 \mathrm{H}), 1.17(\mathrm{ddd}, J=11.7,8.9,3.5 \mathrm{~Hz}, 1 \mathrm{H}), 1.01$ (ddd, $J=12.3,9.1$, $3.4 \mathrm{~Hz}, 1 \mathrm{H}), 0.89$ (s, 3H), 0.80 (s, 3H).

${ }^{13} \mathrm{C}\left\{{ }^{1} \mathrm{H}\right\} \mathrm{NMR}\left(151 \mathrm{MHz}, \mathrm{CDCl}_{3}\right) \delta[\mathrm{ppm}]=144.9,130.7,99.5,84.5,69.2,69.1,67.9,66.9,66.6,61.6$, $61.5,56.9,55.2,51.7,32.2,25.8,20.1,19.8,13.3$. One resonance is missing due solvent overlap.

Synthesis of $(S)-1-A z i d o-2-[3-(R)-(N, N$-dimethylamino)ethyl]ferrocen (2h)

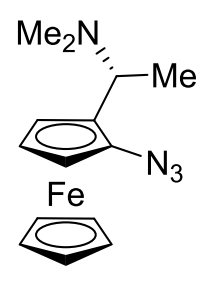

According to GP3, the title compound was prepared from $\mathbf{1 h}$ (76 mg, $0.20 \mathrm{mmol})$. Gravity column chromatography (basic $\mathrm{Al}_{2} \mathrm{O}_{3}$, activity grade $\mathrm{I}+5 \mathrm{wt} \% \mathrm{H}_{2} \mathrm{O} ; \mathrm{CH}_{2} \mathrm{Cl}_{2} / \mathrm{EtOAc}$ 100:0 to 5:1) gave the title compound $2 \mathbf{h}$ (36 mg, $0.12 \mathrm{mmol}, 61 \%$; d.r. $=96: 4\left[{ }^{1} \mathrm{H} \mathrm{NMR}\right]$ ) as a brown oil.

$\mathrm{R}_{\mathrm{f}}=0.50\left(\mathrm{SiO}_{2}, \mathrm{CH}_{2} \mathrm{Cl}_{2} / \mathrm{MeOH} 9: 1\right.$, vis. vanillin) .

${ }^{1} \mathrm{H}$ NMR $\left(500 \mathrm{MHz}, \mathrm{CDCl}_{3}\right) \delta[\mathrm{ppm}]=4.36(\mathrm{dd}, J=2.6,1.5 \mathrm{~Hz}, 1 \mathrm{H}), 4.23(\mathrm{~s}, 5 \mathrm{H}), 4.04(\mathrm{t}, J=2.6 \mathrm{~Hz}$, $1 \mathrm{H}), 4.02(\mathrm{dd}, J=2.8,1.4 \mathrm{~Hz}, 1 \mathrm{H}), 3.75(\mathrm{q}, J=6.9 \mathrm{~Hz}, 1 \mathrm{H}), 2.09$ (s, 6H), 1.44 (d, $J=6.9 \mathrm{~Hz}, 3 \mathrm{H})$.

${ }^{13} \mathrm{C}\left\{{ }^{1} \mathrm{H}\right\} \operatorname{NMR}\left(126 \mathrm{MHz}, \mathrm{CDCl}_{3}\right) \delta[\mathrm{ppm}]=97.9,81.8,69.8,65.2,63.3,59.5,55.2,40.8,15.2$. 


\section{Synthesis of 1-azido-1'-(trimethylsilylethynyl)ferrocene (2i)}

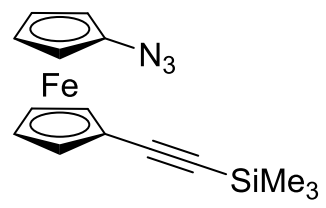

According to GP3, the title compound was prepared from 1i (82 $\mathrm{mg}, 0.20 \mathrm{mmol})$. Gravity column chromatography (basic $\mathrm{Al}_{2} \mathrm{O}_{3}$, activity grade $\mathrm{I}+5$ wt $\% \mathrm{H}_{2} \mathrm{O} ; n$-pentane) gave the title compound $\mathbf{2} \mathbf{i}$ (50 $\mathrm{mg}, 0.15 \mathrm{mmol}, 77 \%)$ as a red oil.

$\mathrm{R}_{\mathrm{f}}=0.57\left(\mathrm{SiO}_{2} ; n\right.$-pentane $/ \mathrm{CH}_{2} \mathrm{Cl}_{2}$ 9:1; vis. vanillin $)$.

${ }^{1} \mathrm{H}$ NMR $\left(700 \mathrm{MHz}, \mathrm{CDCl}_{3}\right) \delta[\mathrm{ppm}]=4.54(\mathrm{t}, J=1.9 \mathrm{~Hz}, 2 \mathrm{H}), 4.26(\mathrm{t}, J=1.9 \mathrm{~Hz}, 2 \mathrm{H}), 4.25(\mathrm{t}$, $J=1.9 \mathrm{~Hz}, 2 \mathrm{H}), 4.07(\mathrm{t}, J=2.0 \mathrm{~Hz}, 2 \mathrm{H}), 0.22(\mathrm{~s}, 9 \mathrm{H})$.

${ }^{13} \mathrm{C}\left\{{ }^{1} \mathrm{H}\right\} \mathrm{NMR}\left(176 \mathrm{MHz}, \mathrm{CDCl}_{3}\right) \delta[\mathrm{ppm}]=103.0,100.3,92.0,73.0,69.8,67.4,66.9,62.4,0.22$.

\section{Synthesis of 1-azido-1'-(ethinylcyclopropyl)ferrocene (2j)}

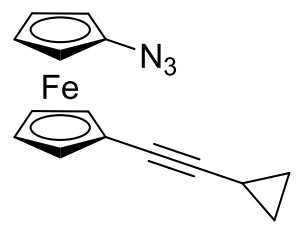

According to GP3, the title compound was prepared from $\mathbf{1 j}$ (75mg, $0.20 \mathrm{mmol}$ ). Gravity column chromatography (basic $\mathrm{Al}_{2} \mathrm{O}_{3}$, activity grade $\mathrm{I}+5 \mathrm{wt} \% \mathrm{H}_{2} \mathrm{O} ; n$-pentane) gave the title compound $\mathbf{2} \mathbf{j}$ (40 mg, $0.14 \mathrm{mmol}, 69 \%)$ as a red oil.

$R_{\mathrm{f}}=0.23\left(\mathrm{SiO}_{2} ; n\right.$-pentane $/ \mathrm{CH}_{2} \mathrm{Cl}_{2} 10: 1 ;$ vis. vanillin $)$.

${ }^{1} \mathrm{H}$ NMR $\left(600 \mathrm{MHz}, \mathrm{CDCl}_{3}\right) \delta[\mathrm{ppm}]=4.45(\mathrm{t}, J=1.9 \mathrm{~Hz}, 2 \mathrm{H}), 4.24(\mathrm{t}, J=1.9 \mathrm{~Hz}, 2 \mathrm{H}), 4.21(\mathrm{t}$, $J=1.9 \mathrm{~Hz}, 2 \mathrm{H}), 4.05(\mathrm{t}, J=2.0 \mathrm{~Hz}, 2 \mathrm{H}), 1.34(\mathrm{tt}, J=8.2,5.0 \mathrm{~Hz}, 1 \mathrm{H}), 0.83-0.79(\mathrm{~m}, 2 \mathrm{H}), 0.77-0.74$ $(\mathrm{m}, 2 \mathrm{H})$.

${ }^{13} \mathrm{C}\left\{{ }^{1} \mathrm{H}\right\}$ NMR $\left(151 \mathrm{MHz}, \mathrm{CDCl}_{3}\right) \delta[\mathrm{ppm}]=99.9,90.7,72.5,69.2,68.4,67.0,62.0,8.5$, 0.5. One resonance is missing presumably due to solvent overlap. 


\section{Synthesis of 1-azido-1'-phenylferrocene (2k)}

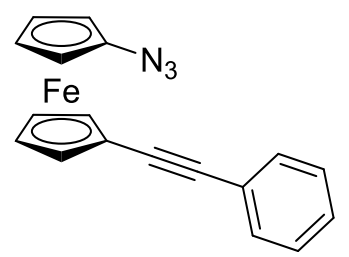

According to GP3, the title compound was prepared from 1k (82 $\mathrm{mg}, 0.20 \mathrm{mmol})$. Gravity column chromatography (basic $\mathrm{Al}_{2} \mathrm{O}_{3}$, activity grade $\mathrm{I}+5 \mathrm{wt} \% \mathrm{H}_{2} \mathrm{O}, n$-pentane) gave the title compound $\mathbf{2 k}$ (51 $\mathrm{mg}, 0.16 \mathrm{mmol}, 78 \%)$ as an orange solid.

$R_{\mathrm{f}}=0.38\left(\mathrm{SiO}_{2} ; n\right.$-pentane $/ \mathrm{CH}_{2} \mathrm{Cl}_{2}$ 9:1, vis. vanillin $)$.

${ }^{1} \mathrm{H}$ NMR $\left(700 \mathrm{MHz}, \mathrm{CDCl}_{3}\right) \delta[\mathrm{ppm}]=7.51-7.47(\mathrm{~m}, 2 \mathrm{H}), 7.34-7.29(\mathrm{~m}, 3 \mathrm{H}), 4.62(\mathrm{t}, J=1.9 \mathrm{~Hz}$, $2 \mathrm{H}), 4.33(\mathrm{t}, J=1.9 \mathrm{~Hz}, 2 \mathrm{H}), 4.31(\mathrm{t}, J=2.0 \mathrm{~Hz}, 2 \mathrm{H}), 4.12(\mathrm{t}, J=1.9 \mathrm{~Hz}, 2 \mathrm{H})$.

${ }^{13} \mathrm{C}\left\{{ }^{1} \mathrm{H}\right\} \mathrm{NMR}\left(176 \mathrm{MHz}, \mathrm{CDCl}_{3}\right) \delta[\mathrm{ppm}]=131.6,128.4,127.9,123.9,100.2,87.2,86.9,72.7,69.9$, 67.3, 62.2. One resonance is missing due solvent overlap.

\section{Synthesis of 1-azido-1'-(1-ethynyl-4-methoxybenzene)ferrocene (2I)}

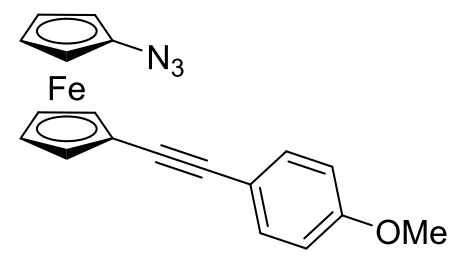

According to GP3, the title compound was prepared from 1k (89 mg, $0.20 \mathrm{mmol})$. Gravity column chromatography (basic $\mathrm{Al}_{2} \mathrm{O}_{3}$, activity grade $\mathrm{I}+5$ wt $\% \mathrm{H}_{2} \mathrm{O} ; n$-pentane $/ \mathrm{CH}_{2} \mathrm{Cl}_{2}$ 10:1) gave the title compound $2 \mathrm{I}$ (53 $\mathrm{mg}, 0.15 \mathrm{mmol}, 74 \%)$ as an orange solid.

$R_{\mathrm{f}}=0.36\left(\mathrm{SiO}_{2}, n\right.$-pentane $/ \mathrm{CH}_{2} \mathrm{Cl}_{2} 4: 1$; vis. vanillin $)$.

${ }^{1} \mathrm{H}$ NMR $\left(700 \mathrm{MHz}, \mathrm{CDCl}_{3}\right) \delta[\mathrm{ppm}]=7.43(\mathrm{~d}, J=8.7 \mathrm{~Hz}, 2 \mathrm{H}), 6.86(\mathrm{~d}, J=8.7 \mathrm{~Hz}, 2 \mathrm{H}), 4.59$ (t, $J=1.9 \mathrm{~Hz}, 2 \mathrm{H}), 4.31(\mathrm{dt}, J=6.0,1.9 \mathrm{~Hz}, 4 \mathrm{H}), 4.11(\mathrm{t}, J=2.0 \mathrm{~Hz}, 2 \mathrm{H}), 3.82(\mathrm{~s}, 3 \mathrm{H})$.

${ }^{13} \mathrm{C}\left\{{ }^{1} \mathrm{H}\right\} \mathrm{NMR}\left(176 \mathrm{MHz}, \mathrm{CDCl}_{3}\right) \delta[\mathrm{ppm}]=159.4,133.0,116.0,114.1,100.2,86.8,85.5,72.5,69.7$, $67.8,67.2,62.2,55.4$. 


\section{Synthesis of 1-azido-1'-(1-ethynyl-4-trifluoromethylbenzene)ferrocene (2m)}

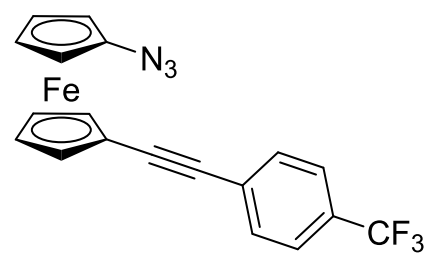

According to GP3, the title compound was prepared from 1m (96 mg, $0.20 \mathrm{mmol})$. Gravity column chromatography (basic $\mathrm{Al}_{2} \mathrm{O}_{3}$, activity grade $\mathrm{I}+5 \mathrm{wt} \% \mathrm{H}_{2} \mathrm{O}, n$-pentane) gave the title compound $\mathbf{2 m}$ (55 mg, $0.14 \mathrm{mmol}, 69 \%$ ) as an orange solid.

$R_{\mathrm{f}}=0.26\left(\mathrm{SiO}_{2} ; n\right.$-pentane, vis. vanillin $)$.

${ }^{1} \mathrm{H}$ NMR $\left(600 \mathrm{MHz}, \mathrm{CDCl}_{3}\right) \delta[\mathrm{ppm}]=7.58(\mathrm{~s}, 4 \mathrm{H}), 4.64(\mathrm{t}, J=1.9 \mathrm{~Hz}, 2 \mathrm{H}), 4.37(\mathrm{t}, J=1.9,2 \mathrm{H}), 4.32$ (t, $J=2.0 \mathrm{~Hz}, 2 \mathrm{H}), 4.13(\mathrm{t}, J=2.0 \mathrm{~Hz}, 2 \mathrm{H})$.

${ }^{13} \mathrm{C}\left\{{ }^{1} \mathrm{H}\right\} \mathrm{NMR}\left(151 \mathrm{MHz}, \mathrm{CDCl}_{3}\right) \delta[\mathrm{ppm}]=131.7,129.6,129.4,127.7,125.4,100.3,90.2,85.7,72.9$, $70.3,67.3,66.4,62.2$. Due to poor resolution of the spectrum, the C-F couplings could not be detected.

\section{Synthesis of 1-azido-1'-(pyridylethynyl)ferrocene (2n)}

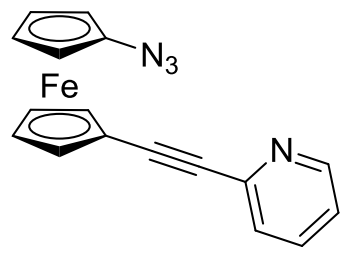

According to GP3, the title compound was prepared from 1n (82 mg, $0.20 \mathrm{mmol})$. Gravity column chromatography (basic $\mathrm{Al}_{2} \mathrm{O}_{3}$, activity grade $\mathrm{I}+5 \mathrm{wt} \% \mathrm{H}_{2} \mathrm{O} ; n$-hexane/EtOAc 10:1) gave the title compound $2 \mathrm{n}$ (40 $\mathrm{mg}, 0.12 \mathrm{mmol}, 62 \%)$ as a brown oil.

$R_{\mathrm{f}}=0.27\left(\mathrm{SiO}_{2} ; n\right.$-hexane/EtOAc $4: 1 ;$ vis. vanillin $)$.

${ }^{1} \mathrm{H}$ NMR $\left(600 \mathrm{MHz}, \mathrm{CDCl}_{3}\right) \delta[\mathrm{ppm}]=8.58(\mathrm{~d}, J=4.8 \mathrm{~Hz}, 1 \mathrm{H}), 7.63(\mathrm{t}, J=7.6 \mathrm{~Hz}, 1 \mathrm{H}), 7.46(\mathrm{~d}$, $J=7.9 \mathrm{~Hz}, 1 \mathrm{H}), 7.20(\mathrm{dd}, J=7.6,4.8 \mathrm{~Hz}, 1 \mathrm{H}), 4.68(\mathrm{~s}, 2 \mathrm{H}), 4.37$ (s, 2H), $4.32(\mathrm{~s}, 2 \mathrm{H}), 4.13(\mathrm{~s}, 2 \mathrm{H})$.

${ }^{13} \mathrm{C}\left\{{ }^{1} \mathrm{H}\right\} \operatorname{NMR}\left(151 \mathrm{MHz}, \mathrm{CDCl}_{3}\right) \delta[\mathrm{ppm}]=150.1,143.9,136.2,126.9,122.4,100.4,88.1,86.4,73.1$, $70.4,67.5,65.7,62.2$. 


\subsection{Synthesis of Ferrocenyl Amines}

\section{General Procedure 5 (GP5) for the Staudinger reduction of ferrocenyl azides}

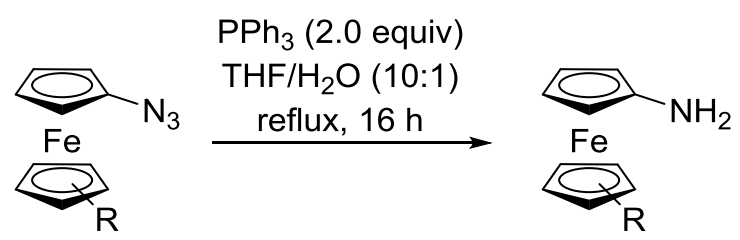

A round bottom flask was charged with ferrocenyl azide (1.0 equiv). THF (10 mL/mmol) and $\mathrm{H}_{2} \mathrm{O}$ ( $1 \mathrm{~mL} / \mathrm{mmol}$ ) were added. To the resulting solution, powdered triphenylphosphine (2.0 equiv) was added in one portion and the mixture was stirred at $\mathrm{rt}$ for $10 \mathrm{~min}$. Then, the mixture was heated to reflux for $16 \mathrm{~h}$ using a reflux condenser. After cooling to $\mathrm{rt}$, the mixture was concentrated under reduced pressure. The residue was dissolved in $\mathrm{CH}_{2} \mathrm{Cl}_{2}$ and loaded onto Celite ${ }^{\circledR}$. Flash column chromatography gave the corresponding amine.

Note 1: Ferrocenyl amines decompose slowly in contact with air and should be stored in the dark under argon at $8{ }^{\circ} \mathrm{C}$.

Note 2: $\mathrm{CDCl}_{3}$ was neutralized by passing it through a small pad of $\mathrm{Na}_{2} \mathrm{CO}_{3}$ prior use. 


\section{Graphical Guide for the Synthesis of Ferrocenyl Amines}

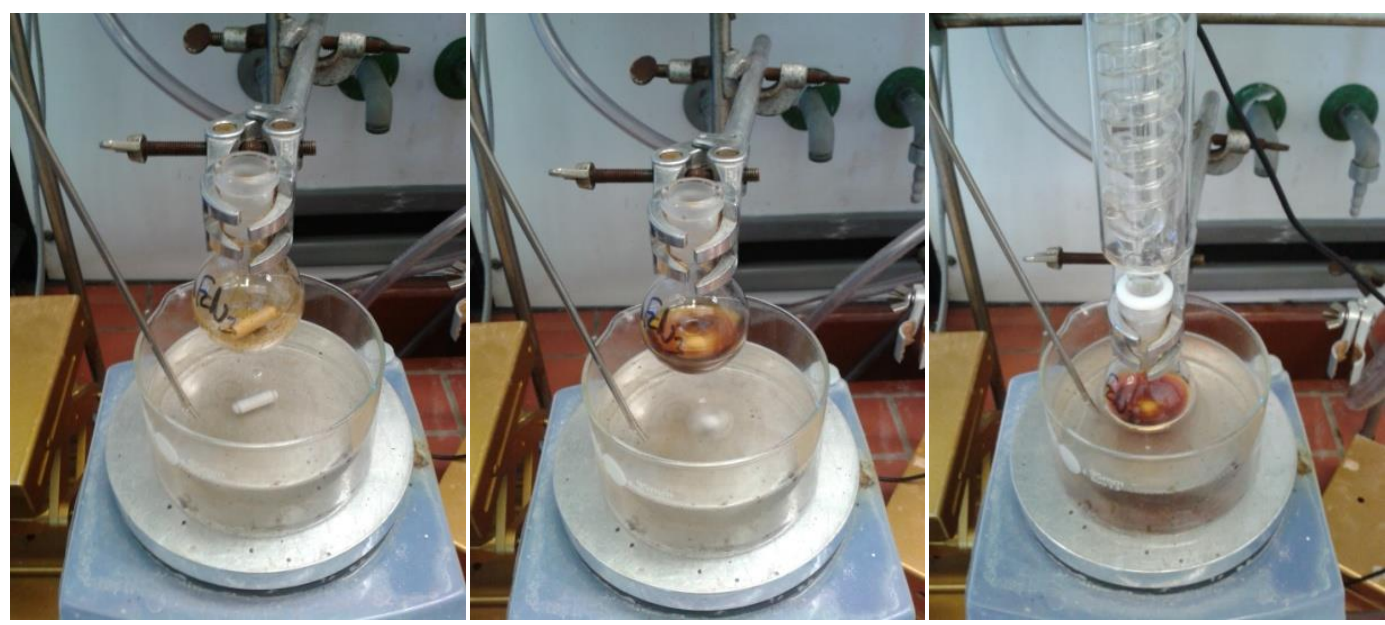

Left: Round bottom flask with ferrocenyl azide. Center: Solution of ferrocenyl azide before the addition of triphenyl phosphine. Right: Reaction mixture after addition of triphenyl phosphine.

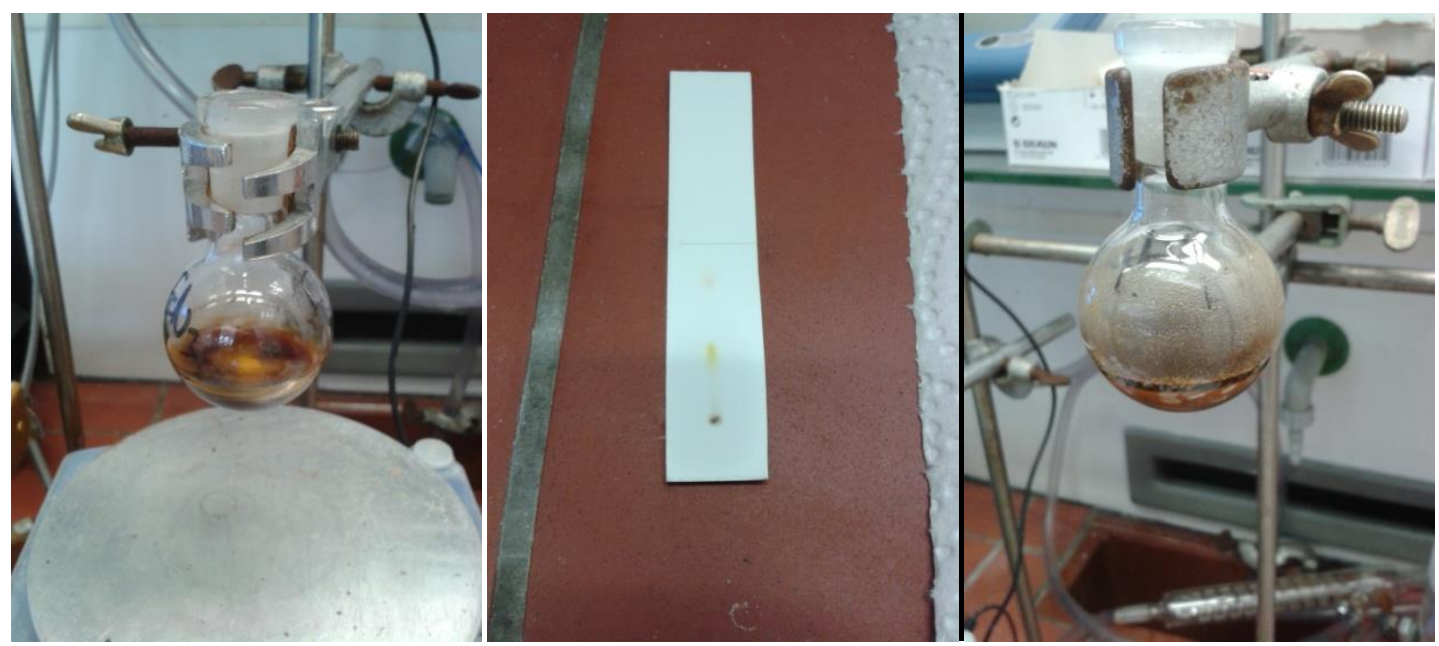

Left: Reaction mixture after refluxing for $16 \mathrm{~h}$. Center: TLC of the crude product. Right: Crude product after addition of Celite ${ }^{\circledR}$.

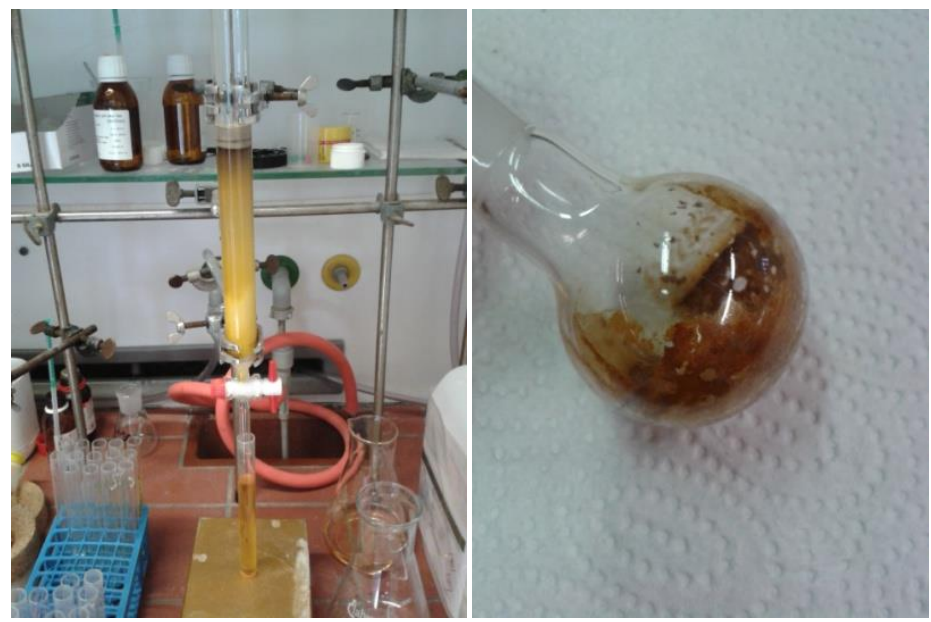

Left: Flash column chromatography of the crude product. Right: Pure product. 


\section{Synthesis of aminoferrocene (5a)}

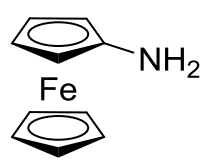

According to GP5, the title compound was prepared from 2a (175 mg, $771 \mu \mathrm{mol}, 1.0$ equiv) and triphenylphosphine (303 mg, $1.16 \mathrm{mmol}, 1.5$ equiv) in THF (5 mL) and $\mathrm{H}_{2} \mathrm{O}(0.5 \mathrm{~mL})$. Flash column chromatography ( $\mathrm{SiO}_{2} ; n$-hexane/EtOAc $\left.1: 1\right)$ gave the title compound $\mathbf{5 a}(131 \mathrm{mg}, 651 \mu \mathrm{mol}, 85 \%)$ as a brown solid.

$R_{\mathrm{f}}=0.42\left(\mathrm{SiO}_{2} ; n\right.$-hexane /EtOAc 1:1; vis. vanillin $)$.

${ }^{1} \mathrm{H} \mathrm{NMR}\left(700 \mathrm{MHz}, \mathrm{CDCl}_{3}\right) \delta[\mathrm{ppm}]=4.10(\mathrm{~s}, 5 \mathrm{H}), 4.00(\mathrm{~s}, 2 \mathrm{H}), 3.85(\mathrm{~s}, 2 \mathrm{H}), 2.59(\mathrm{~s}, 2 \mathrm{H})$.

${ }^{13} \mathrm{C}\left\{{ }^{1} \mathrm{H}\right\} \mathrm{NMR}\left(176 \mathrm{MHz}, \mathrm{CDCl}_{3}\right) \delta[\mathrm{ppm}]=105.5,69.0,63.6,58.0$.

The spectroscopic data are consistent with those reported in the literature. ${ }^{[13]}$

\section{Synthesis of 1-amino-1'-bromoferrocene (5b)}

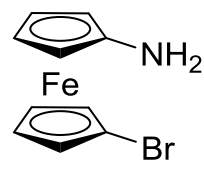

According to GP5, the title compound was prepared from $\mathbf{2 b}$ (100 mg, $0.327 \mathrm{mmol}, 1.0$ equiv) and triphenylphosphine (129 mg, $0.490 \mathrm{mmol}, 1.5$ equiv) in THF $(7 \mathrm{~mL})$ and $\mathrm{H}_{2} \mathrm{O}(0.7 \mathrm{~mL})$. Flash column chromatography $\left(\mathrm{SiO}_{2} ; n\right.$-hexane/EtOAc 3:2) gave the title compound $\mathbf{5 b}(83.0 \mathrm{mg}, 0.297 \mathrm{mmol}, 91 \%)$ as a yellow solid.

$R_{\mathrm{f}}=0.51\left(\mathrm{SiO}_{2} ; n\right.$-hexane/EtOAc 3:2; vis. vanillin)

${ }^{1} \mathrm{H}$ NMR $\left(700 \mathrm{MHz}, \mathrm{C}_{6} \mathrm{D}_{6}\right) \delta[\mathrm{ppm}]=4.13(\mathrm{~s}, 2 \mathrm{H}), 3.72(\mathrm{~s}, 2 \mathrm{H}), 3.69(\mathrm{~s}, 2 \mathrm{H}), 3.59(\mathrm{~s}, 2 \mathrm{H}), 2.09(\mathrm{~s}, 2 \mathrm{H})$. ${ }^{13} \mathrm{C}\left\{{ }^{1} \mathrm{H}\right\}$ NMR $\left(176 \mathrm{MHz}, \mathrm{C}_{6} \mathrm{D}_{6}\right) \delta[\mathrm{ppm}]=108.0,79.9,71.1,67.6,65.8,60.1$.

The spectroscopic data are consistent with those reported in the literature. ${ }^{[14]}$

\section{Synthesis of 1-amino-1'-bromoferrocene $(5 \mathrm{c})$}

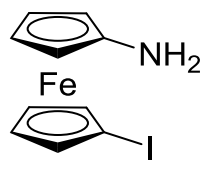

According to GP5, the title compound was prepared from $2 \mathbf{c}$ (50 mg, $0.14 \mathrm{mmol}, 1.0$ equiv) and triphenylphosphine (56 mg, $0.21 \mathrm{mmol}, 1.5$ equiv) in THF $(2 \mathrm{~mL})$ and $\mathrm{H}_{2} \mathrm{O}(0.2 \mathrm{~mL}) . \mathrm{MPLC}\left(\mathrm{SiO}_{2}\right.$; cyclohexane/EtOAc 100:0 to 70:30 to 80:20) gave the title compound $\mathbf{5 c}$ (43 $\mathrm{mg}, 0.13 \mathrm{mmol}, 93 \%$ ) as a yellow solid. 
$R_{\mathrm{f}}=0.45\left(\mathrm{SiO}_{2} ; n\right.$-hexane/EtOAc $2: 1 ;$ vis. vanillin $)$.

${ }^{1} \mathrm{H} \mathrm{NMR}\left(500 \mathrm{MHz}, \mathrm{CDCl}_{3}\right) \delta[\mathrm{ppm}]=4.31(\mathrm{t}, J=1.9 \mathrm{~Hz}, 2 \mathrm{H}), 4.08(\mathrm{t}, J=1.8 \mathrm{~Hz}, 2 \mathrm{H}), 3.91(\mathrm{t}$, $J=1.9 \mathrm{~Hz}, 2 \mathrm{H}), 3.87(\mathrm{t}, J=1.9 \mathrm{~Hz}, 2 \mathrm{H}), 2.72(\mathrm{~s}, 2 \mathrm{H})$.

${ }^{13} \mathrm{C}\left\{{ }^{1} \mathrm{H}\right\} \operatorname{NMR}\left(126 \mathrm{MHz}, \mathrm{CDCl}_{3}\right) \delta[\mathrm{ppm}]=106.7,75.3,69.0,66.1,60.7,44.8$.

HRMS (ESI): $\mathrm{m} / \mathrm{z}$ calcd. for $\mathrm{C}_{10} \mathrm{H}_{10} \mathrm{FeIN}\left([\mathrm{M}]^{+}\right)$326.9207; found: 326.9204 .

\section{Synthesis of 1-amino-1'-ferrocenylcarboxylic acid tert-butyl ester (5d)}

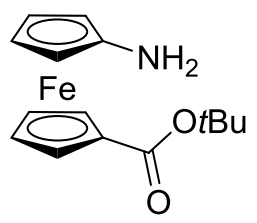

According to GP5, the title compound was prepared from $2 \mathbf{e}$ (150 mg, $458 \mu \mathrm{mol}, 1.0$ equiv) and triphenylphosphine $\left(180 \mathrm{mg}, 688 \mu \mathrm{mol}, 1.5\right.$ equiv) in THF $(3 \mathrm{~mL})$ and $\mathrm{H}_{2} \mathrm{O}(0.3 \mathrm{~mL})$. MPLC (cyclohexane/EtOAc 100:0 to 70:30) gave the title compound 5d (130 mg, $432 \mu \mathrm{mol}, 94 \%$ ) as a red oil. $R_{\mathrm{f}}=0.29\left(\mathrm{SiO}_{2} ; n\right.$-hexane/EtOAc 3:1; vis. vanillin $)$.

${ }^{1} \mathrm{H}$ NMR $\left(500 \mathrm{MHz}, \mathrm{CDCl}_{3}\right) \delta[\mathrm{ppm}]=4.66(\mathrm{t}, J=1.9 \mathrm{~Hz}, 2 \mathrm{H}), 4.29(\mathrm{t}, J=1.9 \mathrm{~Hz}, 2 \mathrm{H}), 3.96(\mathrm{t}$, $J=1.9 \mathrm{~Hz}, 2 \mathrm{H}), 3.87(\mathrm{t}, J=1.9 \mathrm{~Hz}, 2 \mathrm{H}), 2.65(\mathrm{~s}, 2 \mathrm{H}), 1.55(\mathrm{~s}, 9 \mathrm{H})$.

${ }^{13} \mathrm{C}\left\{{ }^{1} \mathrm{H}\right\} \mathrm{NMR}\left(126 \mathrm{MHz}, \mathrm{CDCl}_{3}\right) \delta[\mathrm{ppm}]=170.7,106.6,80.0,74.1,71.6,71.0,65.1,59.6,28.6$.

HRMS (ESI-TOF) m/z calcd. for $\mathrm{C}_{19} \mathrm{H}_{15} \mathrm{~F}_{3} \mathrm{FeN}^{+}\left([\mathrm{M}+\mathrm{H}]^{+}\right)$370.0501; found: 370.0519 .

\section{Synthesis of 1-amino-1'-[(1S, 4S)- 1,7,7-trimethylbicyclo[2.2.1]hept-2-enyl]ferrocene (5e)}

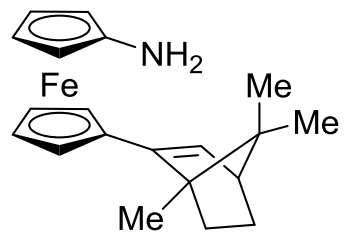

According to GP5, the title compound was prepared from $\mathbf{2 g}$ (50 mg, $0.14 \mathrm{mmol}, 1.0$ equiv) and triphenylphosphine (54 mg, $0.21 \mathrm{mmol}, 1.5$ equiv) in THF (5 mL) and $\mathrm{H}_{2} \mathrm{O}(0.5 \mathrm{~mL})$. MPLC $\left(\mathrm{SiO}_{2}\right.$; cyclohexane/EtOAc 100:0 to 70:30) gave the title compound 5e (39 mg, $0.12 \mathrm{mmol}, 84 \%$ ) as an orange solid.

$R_{\mathrm{f}}=0.31\left(\mathrm{SiO}_{2} ; n\right.$-hexane/EtOAc 10:3; vis. vanillin).

${ }^{1} \mathrm{H} \mathrm{NMR}\left(600 \mathrm{MHz}, \mathrm{CDCl}_{3}\right) \delta[\mathrm{ppm}]=5.95(\mathrm{~d}, J=3.2 \mathrm{~Hz}, 1 \mathrm{H}), 4.21(\mathrm{~s}, 2 \mathrm{H}), 4.12(\mathrm{q}, J=2.3 \mathrm{~Hz}, 2 \mathrm{H})$, $3.92(\mathrm{~d}, J=10.6 \mathrm{~Hz}, 2 \mathrm{H}), 3.83(\mathrm{~s}, 2 \mathrm{H}), 2.55(\mathrm{~s}, 2 \mathrm{H}), 2.29$ (t, $J=3.5 \mathrm{~Hz}, 1 \mathrm{H}), 1.88$ (ddd, $J=12.2,8.5$, $3.8 \mathrm{~Hz}, 1 \mathrm{H}$ ), 1.57 (ddd, $J=12.0,8.6,3.6 \mathrm{~Hz}, 1 \mathrm{H}), 1.25$ (s, 3H), 1.17 (ddd, $J=12.2,9.1,3.7 \mathrm{~Hz}, 1 \mathrm{H}$ ), 1.00 (ddd, $J=12.3,9.1,3.5 \mathrm{~Hz}, 1 \mathrm{H}), 0.90$ (s, 3H), 0.80 (s, 3H). 
${ }^{13} \mathrm{C}\left\{{ }^{1} \mathrm{H}\right\} \mathrm{NMR}\left(151 \mathrm{MHz}, \mathrm{CDCl}_{3}\right) \delta[\mathrm{ppm}]=145.7,129.3,105.5,83.0,69.2,67.7,66.8,64.8,59.7,56.8$, $55.2,51.6,32.2,29.8,26.0,20.2,19.8,13.4$.

HRMS (ESI-TOF) $\mathrm{m} / \mathrm{z}$ calcd. for $\mathrm{C}_{20} \mathrm{H}_{25} \mathrm{FeN}^{+}\left([\mathrm{M}]^{+}\right)$335.1336; found: 335.1339 .

$[\alpha]_{\mathrm{D}}^{24}=-9.0\left(c=1.00, \mathrm{CHCl}_{3}\right)$.

Synthesis of 1-amino-1'-(trimethylsilylethynyl)ferrocene (5f)

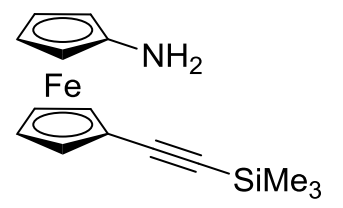

According to GP5, the title compound was prepared from $2 \mathbf{i}$ (20 mg, $0.062 \mathrm{mmol}, 1.0$ equiv) and triphenylphosphine (49 mg, $0.19 \mathrm{mmol}, 3.0$ equiv) in THF $(1.5 \mathrm{~mL})$ and $\mathrm{H}_{2} \mathrm{O}(0.15 \mathrm{~mL})$. Flash column chromatography $\left(\mathrm{SiO}_{2} ; n\right.$-pentane/EtOAc/Et $3 \mathrm{~N}$ 150:10:1) gave the title compound 5 f $(14 \mathrm{mg}$, $0.047 \mathrm{mmol}, 76 \%)$ as a dark yellow solid.

$R_{\mathrm{f}}=0.31\left(\mathrm{SiO}_{2} ; n\right.$-pentane/EtOAc/Et ${ }_{3} \mathrm{~N}$ 90:10:1; vis. vanillin $)$.

${ }^{1} \mathrm{H}$ NMR $\left(400 \mathrm{MHz}, \mathrm{CDCl}_{3}\right) \delta[\mathrm{ppm}]=4.38(\mathrm{t}, J=1.9 \mathrm{~Hz}, 2 \mathrm{H}), 4.14(\mathrm{t}, J=1.9 \mathrm{~Hz}, 2 \mathrm{H}), 3.93(\mathrm{t}$, $J=1.9 \mathrm{~Hz}, 2 \mathrm{H}), 3.87(\mathrm{t}, J=1.9 \mathrm{~Hz}, 2 \mathrm{H}), 2.74(\mathrm{~s}, 2 \mathrm{H}), 0.22(\mathrm{~s}, 9 \mathrm{H})$.

${ }^{13} \mathrm{C}\left\{{ }^{1} \mathrm{H}\right\}$ NMR $\left(100 \mathrm{MHz}, \mathrm{CDCl}_{3}\right) \delta[\mathrm{ppm}]=104.1,104.0,91.2,72.7,69.1,66.0,65.4,60.1,0.3$.

HRMS (ESI): $\mathrm{m} / \mathrm{z}$ calcd. for $\mathrm{C}_{15} \mathrm{H}_{19} \mathrm{FeNSi}\left([\mathrm{M}]^{+}\right)$297.0636; found: 297.0637 .

Note: For TLC and column chromatography, the silica was deactivated prior to use with the stated eluent containing $\mathrm{Et}_{3} \mathrm{~N}$.

Synthesis of 1-amino-1'-(ethinylcyclopropyl)ferrocene (5g)

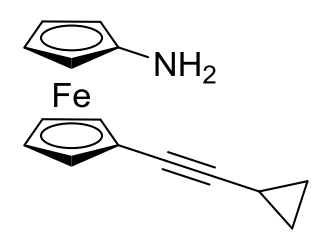

According to GP5, the title compound was prepared from $\mathbf{2 j}$ (30 $\mathrm{mg}, 0.10 \mathrm{mmol}, 1.0$ equiv) and triphenylphosphine (41 mg, $0.16 \mathrm{mmol}, 3.0$ equiv) in THF (2 mL) and $\mathrm{H}_{2} \mathrm{O}(0.2 \mathrm{~mL})$. Flash column chromatography $\left(\mathrm{SiO}_{2} ; n\right.$-hexane/EtOAc 10:3) gave the title compound $\mathbf{5 g}$ (22 $\mathbf{m g}, 0.080 \mathrm{mmol}, 81 \%$ ) as a brown solid.

$R_{\mathrm{f}}=0.33\left(\mathrm{SiO}_{2} ; n\right.$-hexane/EtOAc 10:3; vis. vanillin) .

${ }^{1} \mathrm{H} \mathrm{NMR}\left(500 \mathrm{MHz}, \mathrm{CDCl}_{3}\right) \delta[\mathrm{ppm}]=4.29(\mathrm{~s}, 2 \mathrm{H}), 4.07$ (s, 2H), $3.93(\mathrm{~s}, 2 \mathrm{H}), 3.85(\mathrm{~s}, 2 \mathrm{H}), 2.75$ (s, $2 \mathrm{H}), 1.36$ (dt, $J=8.4,5.2,1 \mathrm{H}), 0.83(\mathrm{dt}, J=7.0,3.5,2 \mathrm{H}), 0.72$ (dd, $J=5.1,2.3,2 \mathrm{H}$ ).

${ }^{13} \mathrm{C}\left\{{ }^{1} \mathrm{H}\right\}$ NMR $\left(126 \mathrm{MHz}, \mathrm{CDCl}_{3}\right) \delta[\mathrm{ppm}]=104.5,90.6,73.2,72.2,68.6,67.3,65.1,60.2,8.7,0.4$. 
HRMS (ESI-TOF) m/z calcd. for $\mathrm{C}_{15} \mathrm{H}_{16} \mathrm{FeN}^{+}\left([\mathrm{M}+\mathrm{H}]^{+}\right)$266.0627; found: 266.0635 .

\section{Synthesis of 1-amino-1'-(phenylethynyl)ferrocene (5h)}

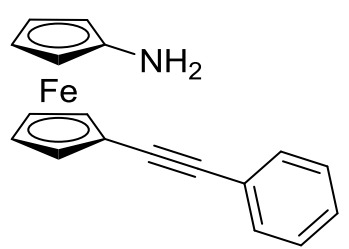

According to GP5, the title compound was prepared from $\mathbf{2 k}$ (25 mg, $0.076 \mathrm{mmol}, 1.0$ equiv) and triphenylphosphine (60 mg, $0.23 \mathrm{mmol}, 3.0$ equiv) in THF $(2 \mathrm{~mL})$ and $\mathrm{H}_{2} \mathrm{O}(0.2 \mathrm{~mL})$. Flash column chromatography $\left(\mathrm{SiO}_{2} ; n\right.$-hexane/EtOAc 7:3) followed by recrystallization from $n$-pentane at $-78{ }^{\circ} \mathrm{C}$ gave the title compound $\mathbf{5 h}(18 \mathrm{mg}, 0.060 \mathrm{mmol}, 78 \%)$ as an orange solid.

$R_{\mathrm{f}}=0.36\left(\mathrm{SiO}_{2} ; n\right.$-hexane/EtOAc 7:3; vis. vanillin $)$.

${ }^{1} \mathrm{H}$ NMR $\left(400 \mathrm{MHz}, \mathrm{CDCl}_{3}\right) \delta[\mathrm{ppm}]=7.49-7.45(\mathrm{~m}, 2 \mathrm{H}), 7.36-7.29(\mathrm{~m}, 3 \mathrm{H}), 4.46(\mathrm{t}, J=1.9 \mathrm{~Hz}$, $2 \mathrm{H}), 4.20(\mathrm{t}, J=1.9 \mathrm{~Hz}, 2 \mathrm{H}), 4.00(\mathrm{t}, J=1.9 \mathrm{~Hz}, 2 \mathrm{H}), 3.90(\mathrm{t}, J=1.9 \mathrm{~Hz}, 2 \mathrm{H}), 2.74(\mathrm{~s}, 2 \mathrm{H})$.

${ }^{13} \mathrm{C}\left\{{ }^{1} \mathrm{H}\right\} \mathrm{NMR}\left(100 \mathrm{MHz}, \mathrm{CDCl}_{3}\right) \delta[\mathrm{ppm}]=131.4,128.6,127.9,123.9,105.4,88.1,86.9,72.4,69.3$, $66.3,65.3,60.1$.

HRMS (ESI): $\mathrm{m} / \mathrm{z}$ calcd. for $\mathrm{C}_{18} \mathrm{H}_{15} \mathrm{FeN}^{+}\left([\mathrm{M}]^{+}\right)$301.0554; found: 301.0555 .

\section{Synthesis of 1-amino-1'-(ethinylcyclopropyl)ferrocene (5i)}

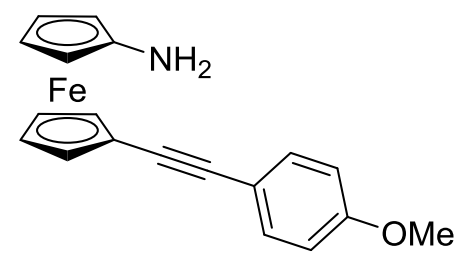

According to GP5, the title compound was prepared from 21 (40 mg, $0.11 \mathrm{mmol}, 1.0$ equiv) and triphenylphosphine ( $38 \mathrm{mg}, 0.17 \mathrm{mmol}, 1.5$ equiv) in THF $(2 \mathrm{~mL})$ and $\mathrm{H}_{2} \mathrm{O}(0.2 \mathrm{~mL})$. Flash column chromatography ( $\mathrm{SiO}_{2}$; $n$-hexane/EtOAc 3:1) gave the title compound $\mathbf{5 i}(28 \mathrm{mg}, 0.085 \mathrm{mmol}, 75 \%)$ as an orange solid.

$R_{\mathrm{f}}=0.30\left(\mathrm{SiO}_{2} ; n\right.$-hexane/EtOAc 3:1; vis. vanillin $)$.

${ }^{1} \mathrm{H}$ NMR $\left(600 \mathrm{MHz}, \mathrm{CDCl}_{3}\right) \delta[\mathrm{ppm}]=7.40(\mathrm{~d}, J=8.7 \mathrm{~Hz}, 2 \mathrm{H}), 6.85(\mathrm{~d}, J=8.7 \mathrm{~Hz}, 2 \mathrm{H}), 4.44(\mathrm{~s}, 2 \mathrm{H})$, 4.18 (s, 2H), 4.00 (s, 2H), 3.90 (s, 2H), 3.82 (s, 3H), 2.74 (s, 2H).

${ }^{13} \mathrm{C}\left\{{ }^{1} \mathrm{H}\right\}$ NMR $\left(151 \mathrm{MHz}, \mathrm{CDCl}_{3}\right) \delta[\mathrm{ppm}]=159.4,132.8,116.0,114.2,105.2,86.7,86.2,72.2,69.1$, $66.8,65.3,60.1,55.5$.

HRMS (ESI-TOF) m/z calcd. for $\mathrm{C}_{19} \mathrm{H}_{18} \mathrm{FeNO}^{+}\left([\mathrm{M}+\mathrm{H}]^{+}\right)$332.0733; found: 332.0733 . 


\section{Synthesis of 1-amino-1'-[(4-trifluoromethyl)phenylethynyl]ferrocene (5j)}

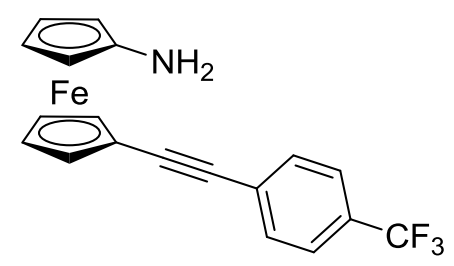

According to GP5, the title compound was prepared from $\mathbf{2 m}$ (100 mg, $253 \mu \mathrm{mol}, 1.0$ equiv) and triphenylphosphine $\left(85.9 \mathrm{mg}, 380 \mu \mathrm{mol}, 1.5\right.$ equiv) in THF $(5 \mathrm{~mL})$ and $\mathrm{H}_{2} \mathrm{O}(0.5 \mathrm{~mL})$. MPLC (cyclohexane/EtOAc 90:10 to 70:3) gave the title compound $\mathbf{5 j}(85.0 \mathrm{mg}, 230 \mu \mathrm{mol}, 91 \%$ ) as an orange solid.

$R_{\mathrm{f}}=0.41\left(\mathrm{SiO}_{2} ; n\right.$-hexane/EtOAc 3:1; vis. vanillin $)$.

${ }^{1} \mathrm{H}$ NMR $\left(500 \mathrm{MHz}, \mathrm{CD}_{2} \mathrm{Cl}_{2}\right) \delta[\mathrm{ppm}]=7.59(\mathrm{~s}, 4 \mathrm{H}), 4.48(\mathrm{t}, J=1.9 \mathrm{~Hz}, 2 \mathrm{H}), 4.24(\mathrm{t}, J=1.9 \mathrm{~Hz}, 2 \mathrm{H})$, 3.99 (t, $J=1.9 \mathrm{~Hz}, 2 \mathrm{H}), 3.90(\mathrm{t}, J=1.9 \mathrm{~Hz}, 2 \mathrm{H}), 2.72(\mathrm{~s}, 2 \mathrm{H})$.

${ }^{13} \mathrm{C}\left\{{ }^{1} \mathrm{H}\right\} \mathrm{NMR}\left(126 \mathrm{MHz}, \mathrm{CD}_{2} \mathrm{Cl}_{2}\right) \delta[\mathrm{ppm}]=131.9,129.5(\mathrm{q}, J=32.5 \mathrm{~Hz}), 128.6,125.9(\mathrm{q}, J=3.8 \mathrm{~Hz})$, $123.7,107.1,92.1,85.7,73.0,70.3,65.8,65.5,60.0$. Due to poor resolution of the spectrum, one C-F coupling could not be detected.

${ }^{19} \mathrm{~F}$ NMR $\left(471 \mathrm{MHz}, \mathrm{CD}_{2} \mathrm{Cl}_{2}\right) \delta[\mathrm{ppm}]=-62.9$.

HRMS (ESI-TOF) m/z calcd. for $\mathrm{C}_{19} \mathrm{H}_{15} \mathrm{~F}_{3} \mathrm{FeN}^{+}\left([\mathrm{M}+\mathrm{H}]^{+}\right) 370.0501$; found: 370.0519 . 


\section{NMR Spectra of Synthesized Compounds}

\subsection{NMR Spectra of Compounds Synthesized in Batch}

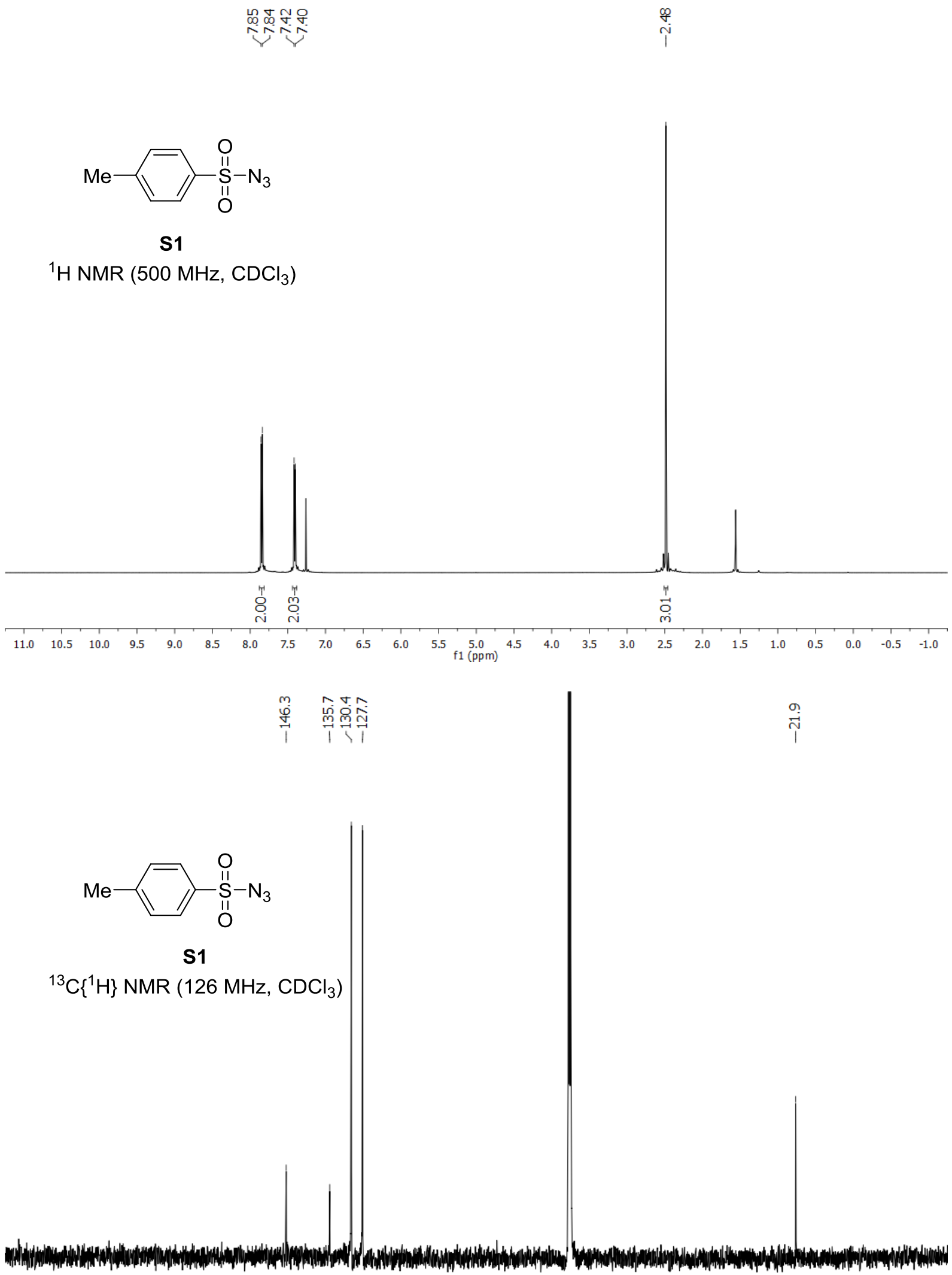

$\begin{array}{llllllllllllllllllllllll}210 & 200 & 190 & 180 & 170 & 160 & 150 & 140 & 130 & 120 & 110 & 100 & 90 & 80 & 70 & 60 & 50 & 40 & 30 & 20 & 10 & 0 & -10\end{array}$ 


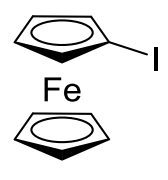

$1 a$

${ }^{1} \mathrm{H}$ NMR (500 MHz, $\left.\mathrm{CDCl}_{3}\right)$

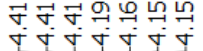 \\ idiga}
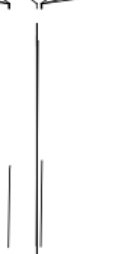

ชูำ
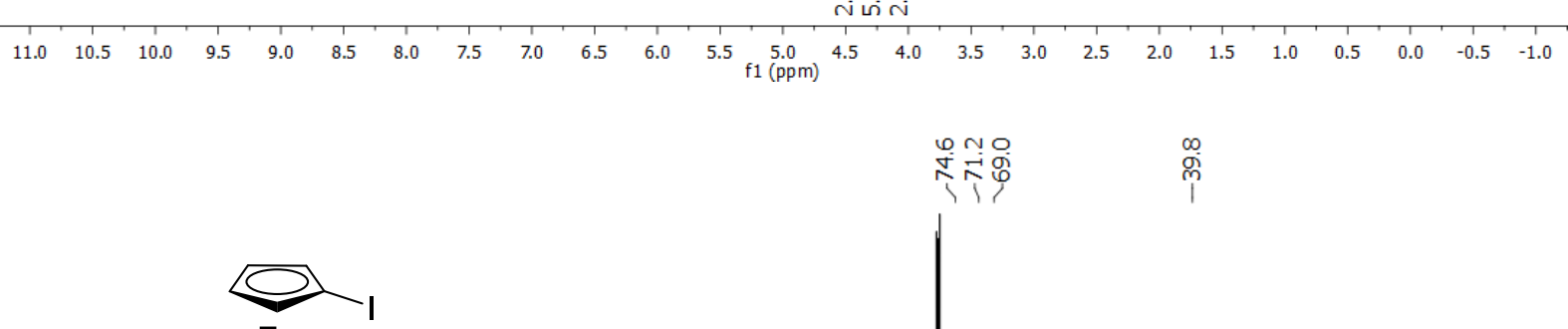

$\mathrm{Fe}$

$1 \mathrm{a}$

${ }^{13} \mathrm{C}\left\{{ }^{1} \mathrm{H}\right\}$ NMR $\left(126 \mathrm{MHz}, \mathrm{CDCl}_{3}\right)$

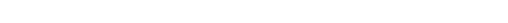

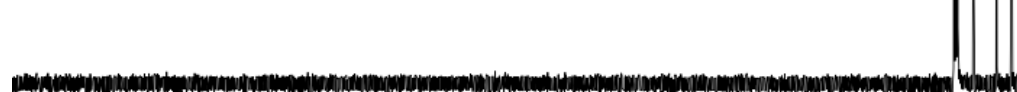

\begin{tabular}{|c|c|c|c|c|c|c|c|c|c|c|c|c|c|c|c|c|c|c|c|c|c|c|}
\hline 210 & 200 & 190 & 180 & 170 & 160 & 150 & 140 & 130 & 120 & 110 & $\begin{array}{c}100 \\
\mathrm{f} 1(\mathrm{ppm})\end{array}$ & 90 & 80 & 70 & 60 & 50 & 40 & 30 & 20 & 10 & 0 & -10 \\
\hline
\end{tabular}




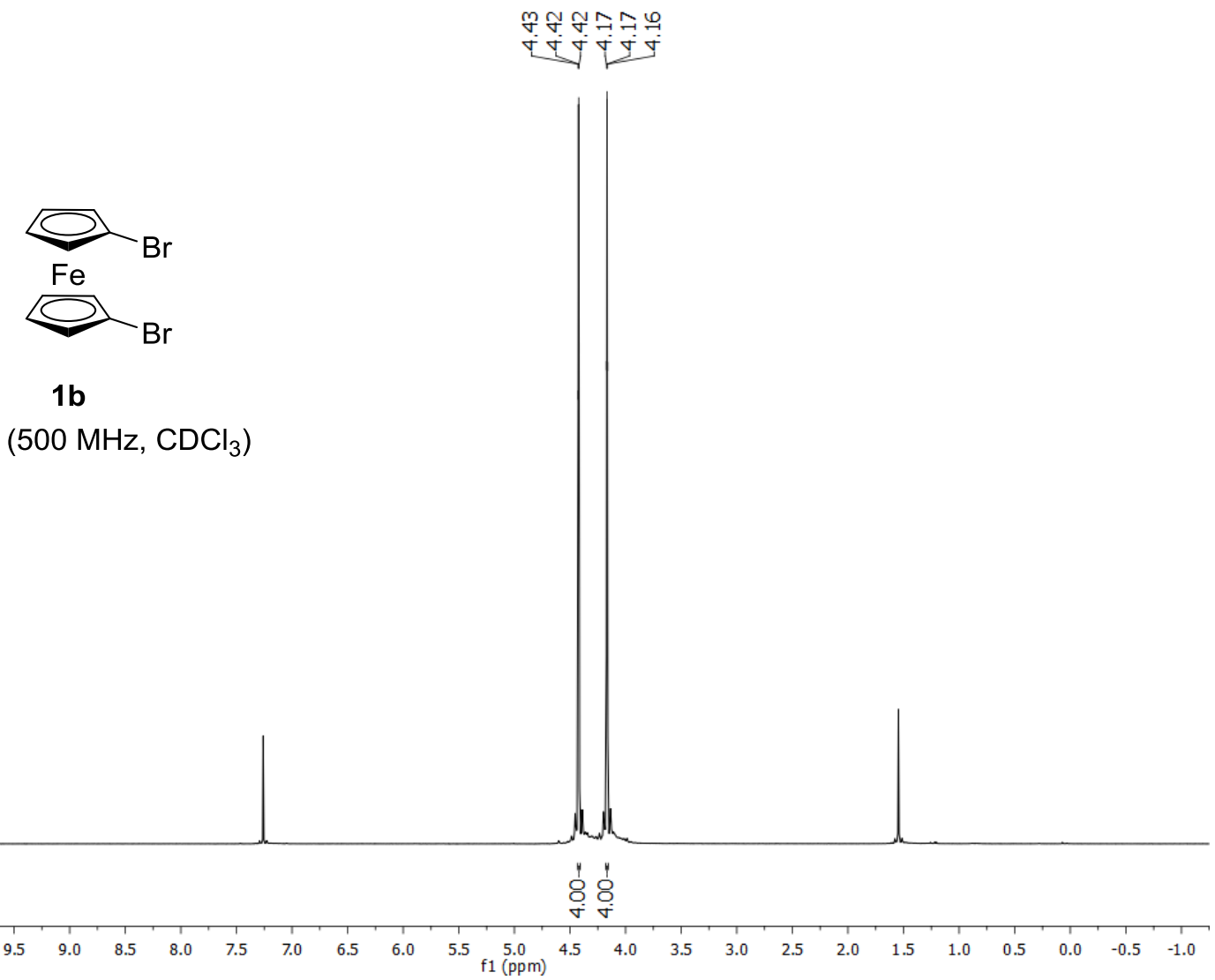

当



$1 \mathrm{~b}$

${ }^{13} \mathrm{C}\left\{{ }^{1} \mathrm{H}\right\}$ NMR $\left(126 \mathrm{MHz}, \mathrm{CDCl}_{3}\right)$

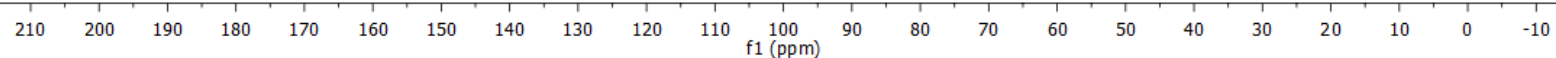




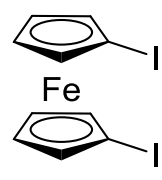

$1 c$

${ }^{1} \mathrm{H}$ NMR $\left(700 \mathrm{MHz}, \mathrm{CDCl}_{3}\right.$ )

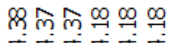

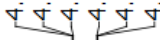

1HNMR (700 $\left.\mathrm{MHz}, \mathrm{CDCl}_{3}\right)$

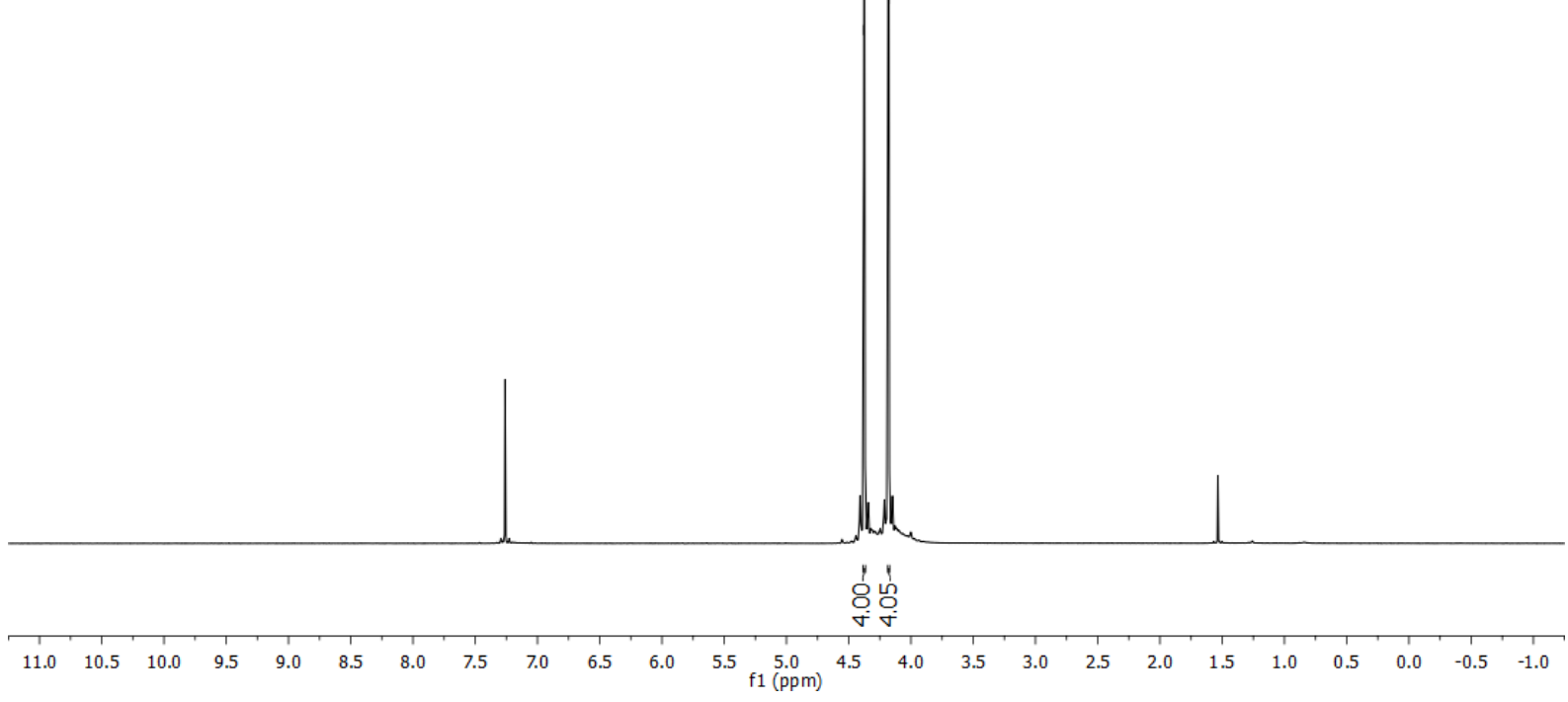

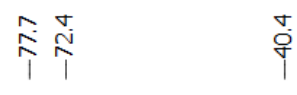

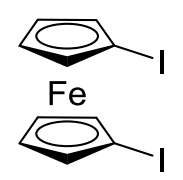

1c

${ }^{13} \mathrm{C}\left\{{ }^{1} \mathrm{H}\right\} \operatorname{NMR}\left(176 \mathrm{MHz}, \mathrm{CDCl}_{3}\right)$

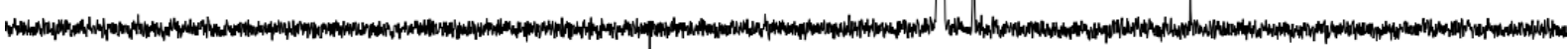

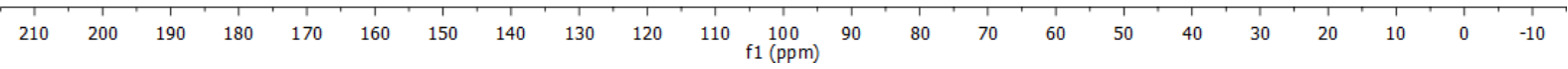




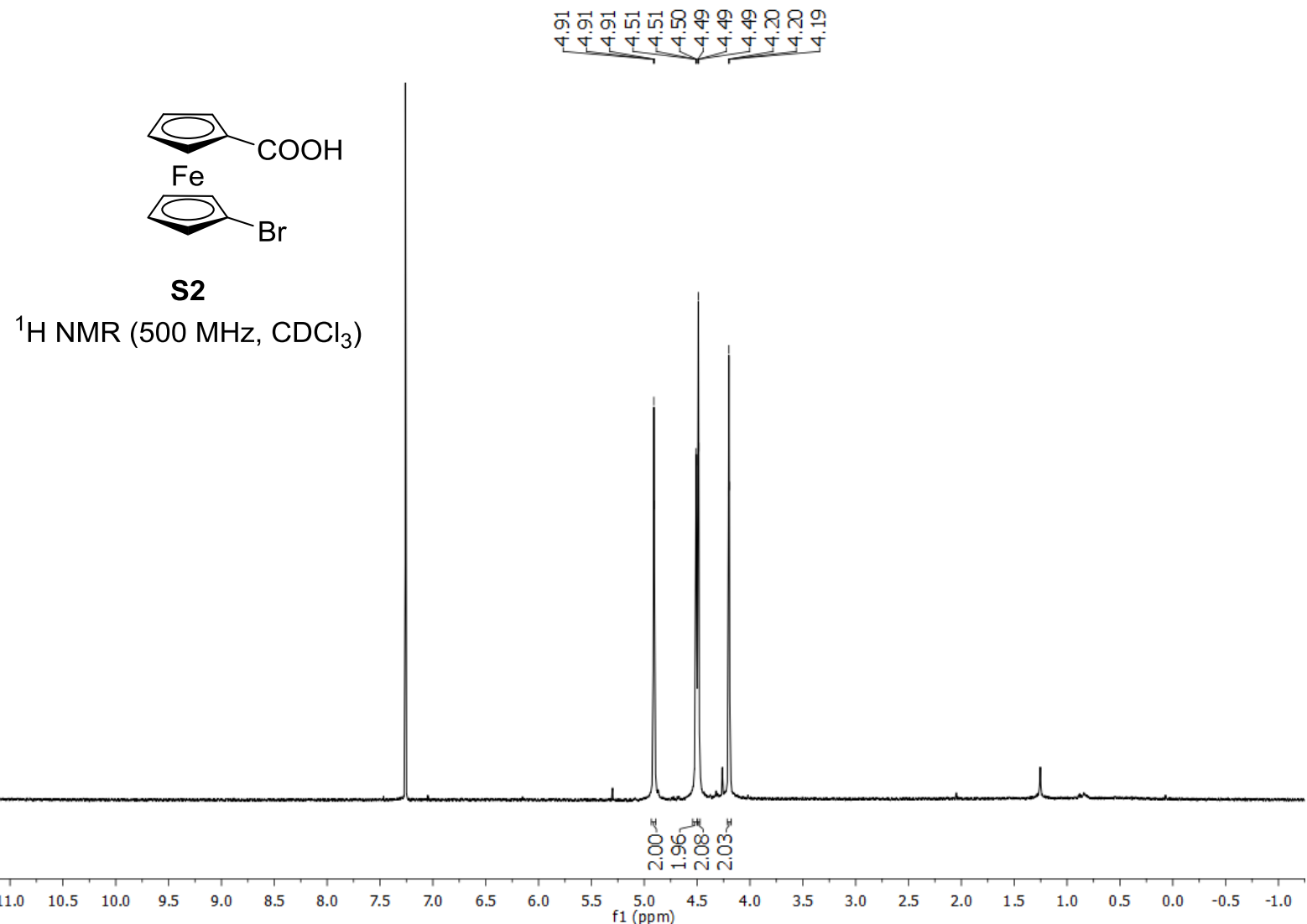

8ุ

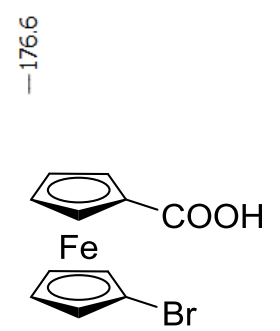

S2

${ }^{13} \mathrm{C}\left\{{ }^{1} \mathrm{H}\right\}$ NMR $\left(176 \mathrm{MHz}, \mathrm{CDCl}_{3}\right)$

$\begin{array}{llllllllllll}210 & 200 & 190 & 180 & 170 & 160 & 150 & 140 & 130 & 120 & 110 & \underset{f 1}{100}(\mathrm{ppm})\end{array}$

$\underset{n}{n}$

. 


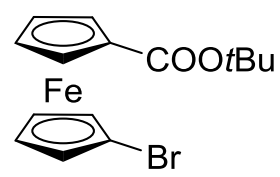

$1 e$

${ }^{1} \mathrm{H}$ NMR $\left.\left(700 \mathrm{MHz}^{\mathrm{CDCl}}\right)_{3}\right)$

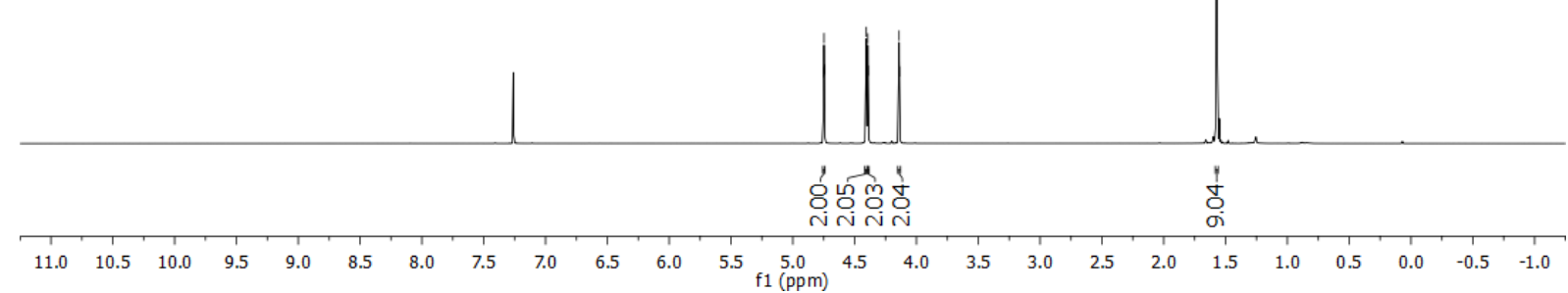

$\frac{0}{i}$

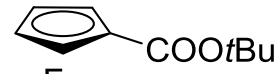

$\mathrm{Fe}$

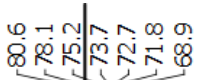

$\stackrel{\substack{n \\ \infty}}{1}$

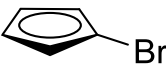

$1 e$

${ }^{13} \mathrm{C}\left\{{ }^{1} \mathrm{H}\right\} \operatorname{NMR}\left(176 \mathrm{MHz}, \mathrm{CDCl}_{3}\right)$

$\begin{array}{lllllllllll}210 & 200 & 190 & 180 & 170 & 160 & 150 & 140 & 130 & 120 & 110 \\ \mathrm{f} 1(\mathrm{ppm})\end{array}$ 

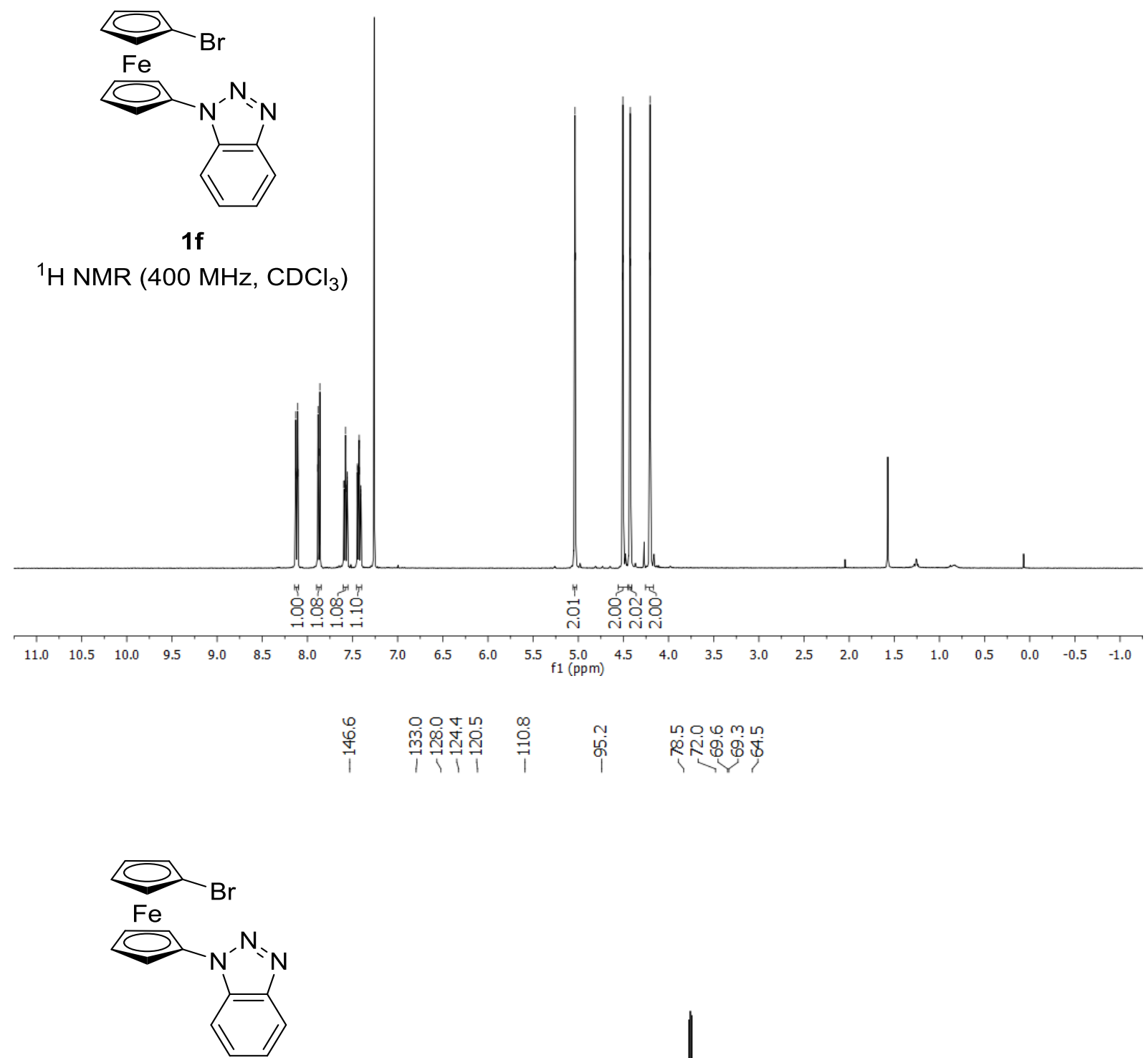

$1 f$

${ }^{13} \mathrm{C}\left\{{ }^{1} \mathrm{H}\right\}$ NMR (100 MHz, $\left.\mathrm{CDCl}_{3}\right)$

$\begin{array}{llllllllllll}210 & 200 & 190 & 180 & 170 & 160 & 150 & 140 & 130 & 120 & 110 & 100\end{array}$ 


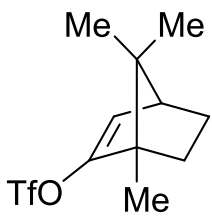

S3

${ }^{1} \mathrm{H} \mathrm{NMR}\left(500 \mathrm{MHz}, \mathrm{CDCl}_{3}\right)$
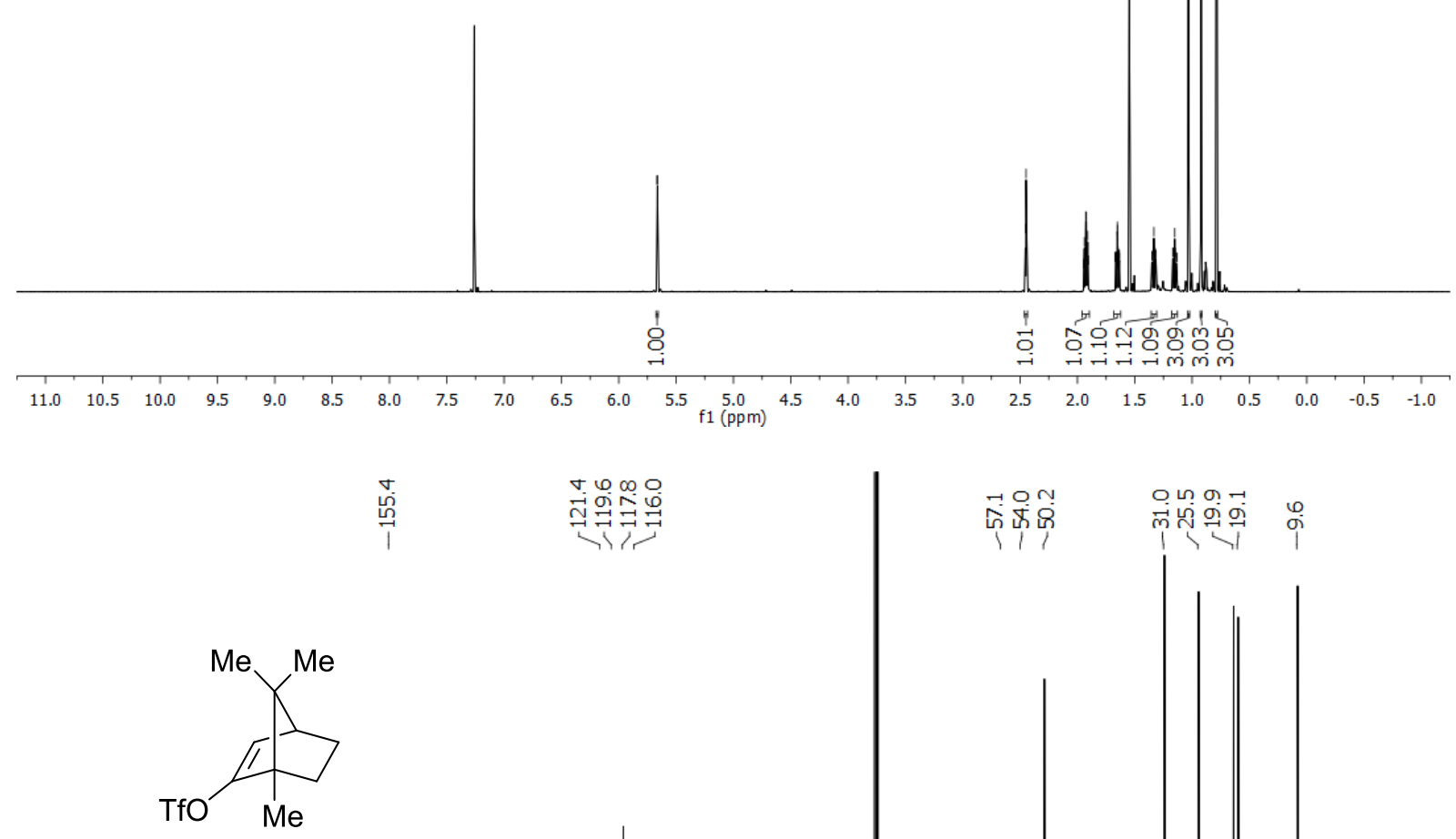

S3

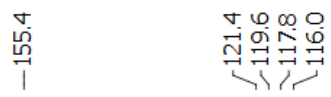

กิ่

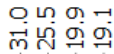

तर

${ }^{13} \mathrm{C}\left\{{ }^{1} \mathrm{H}\right\}$ NMR $\left(176 \mathrm{MHz}, \mathrm{CDCl}_{3}\right)$ 


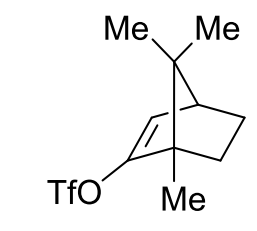

S3

${ }^{19} \mathrm{~F} \mathrm{NMR}\left(565 \mathrm{MHz}, \mathrm{CDCl}_{3}\right)$

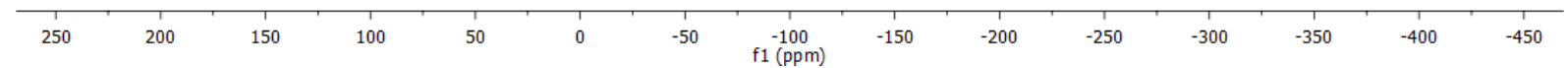




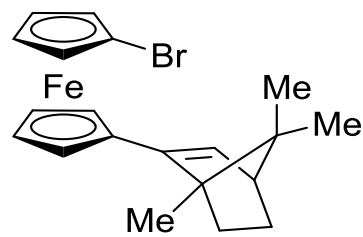

$1 \mathrm{~g}$

${ }^{1} \mathrm{H}$ NMR $\left(500 \mathrm{MHz}, \mathrm{CDCl}_{3}\right)$

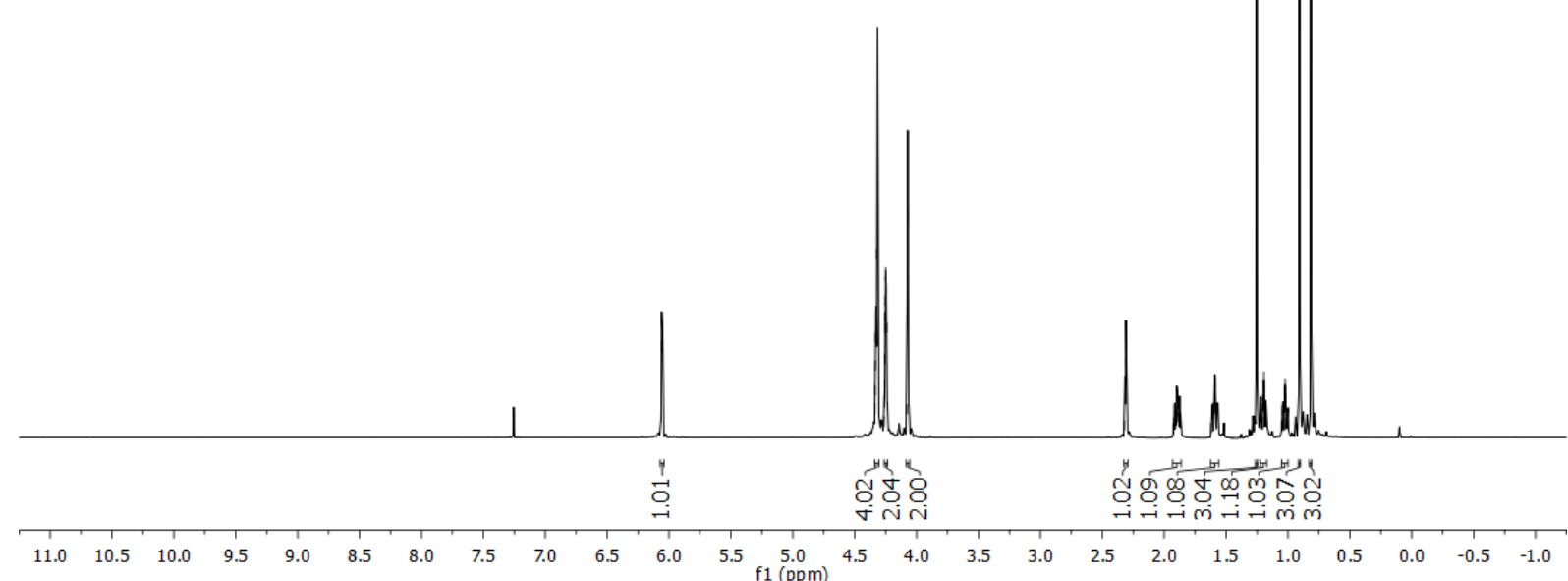

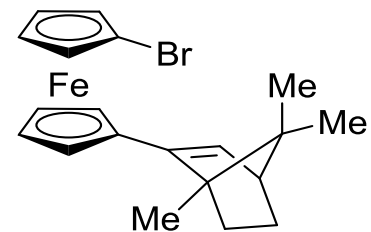

$1 \mathrm{~g}$

${ }^{13} \mathrm{C}\left\{{ }^{1} \mathrm{H}\right\}$ NMR $\left.(126 \mathrm{MHz}, \mathrm{CDCl})_{3}\right)$

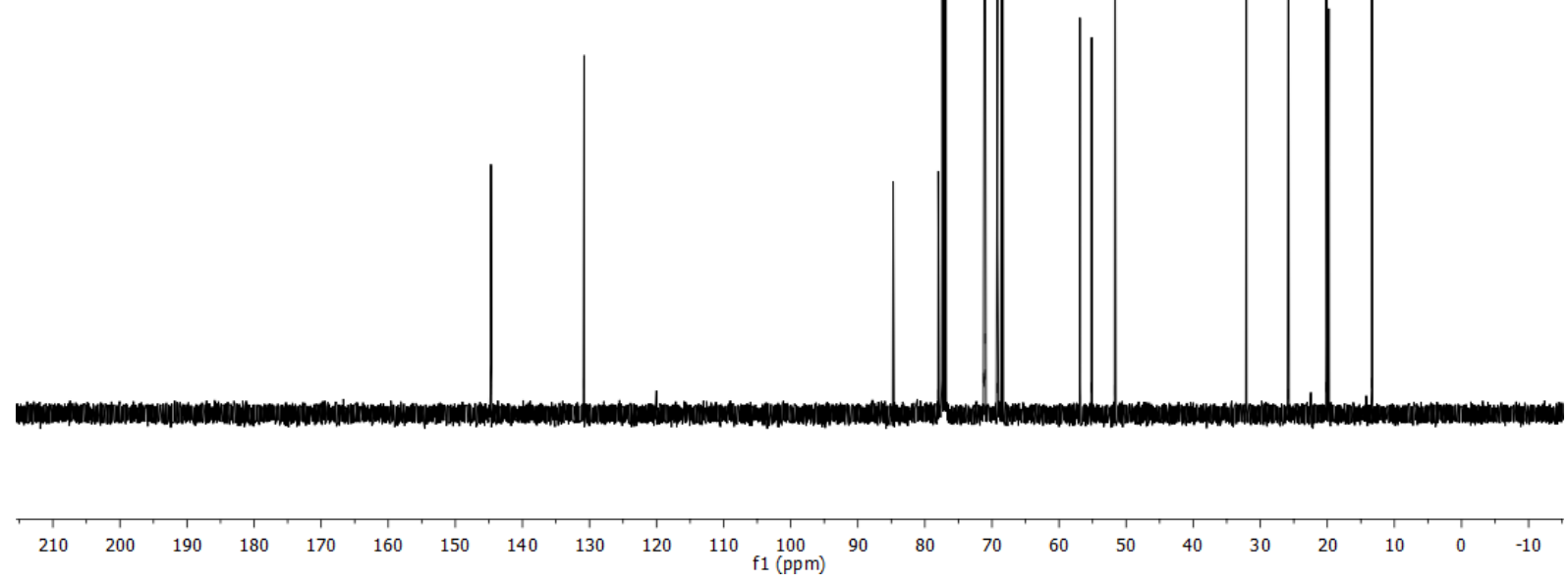




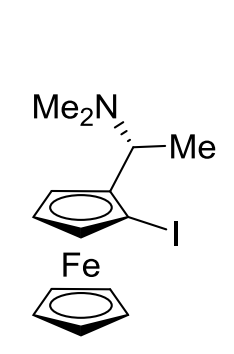

$1 \mathrm{~h}$

${ }^{1} \mathrm{H}$ NMR $\left(500 \mathrm{MHz}, \mathrm{CDCl}_{3}\right)$
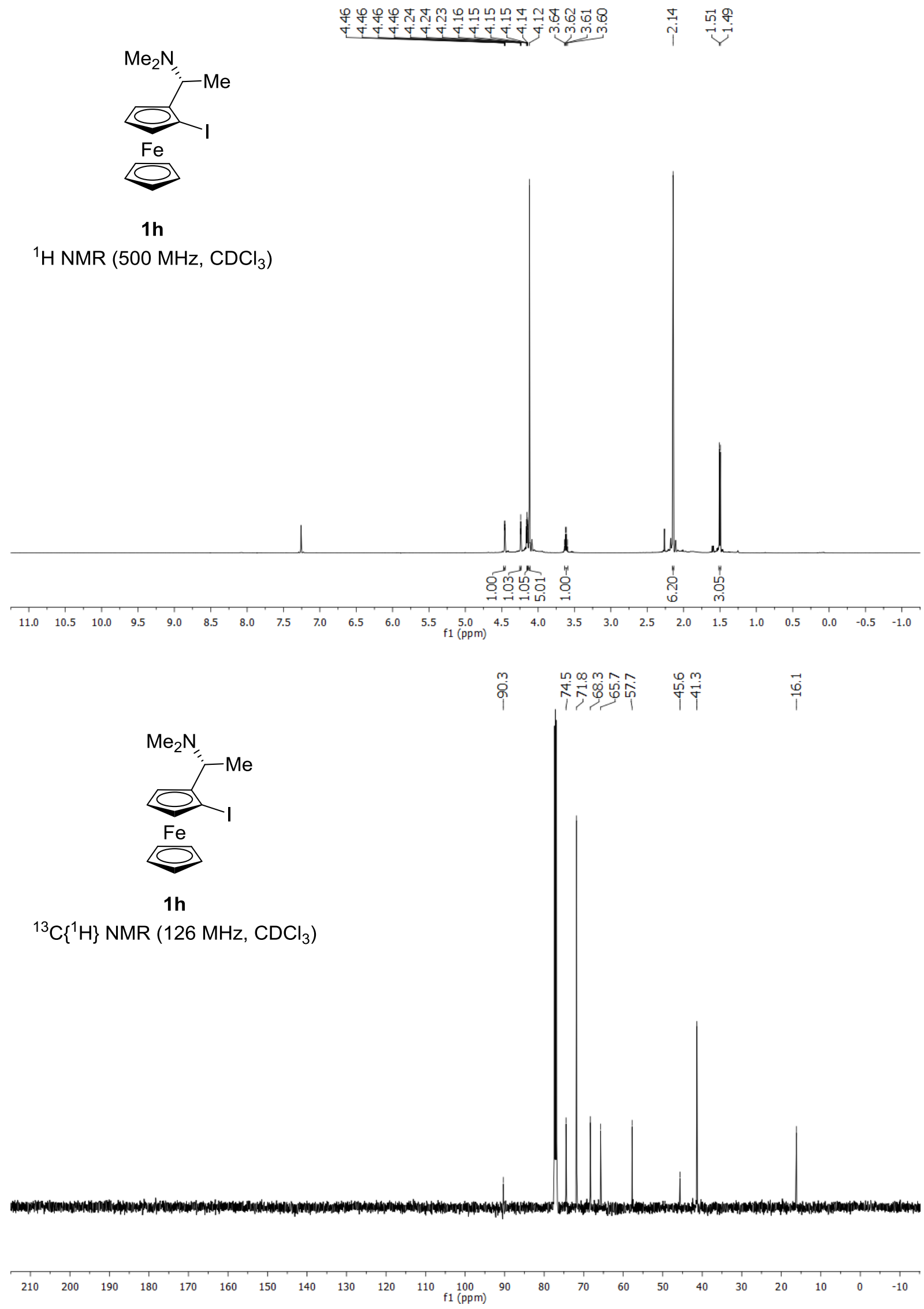

$1 \mathrm{~h}$

${ }^{13} \mathrm{C}\left\{{ }^{1} \mathrm{H}\right\}$ NMR $\left(126 \mathrm{MHz}, \mathrm{CDCl}_{3}\right)$

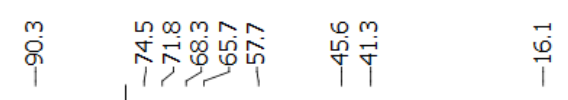




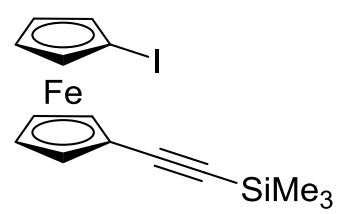

$1 \mathrm{i}$

${ }^{1} \mathrm{H}$ NMR $\left(700 \mathrm{MHz}, \mathrm{CDCl}_{3}\right)$
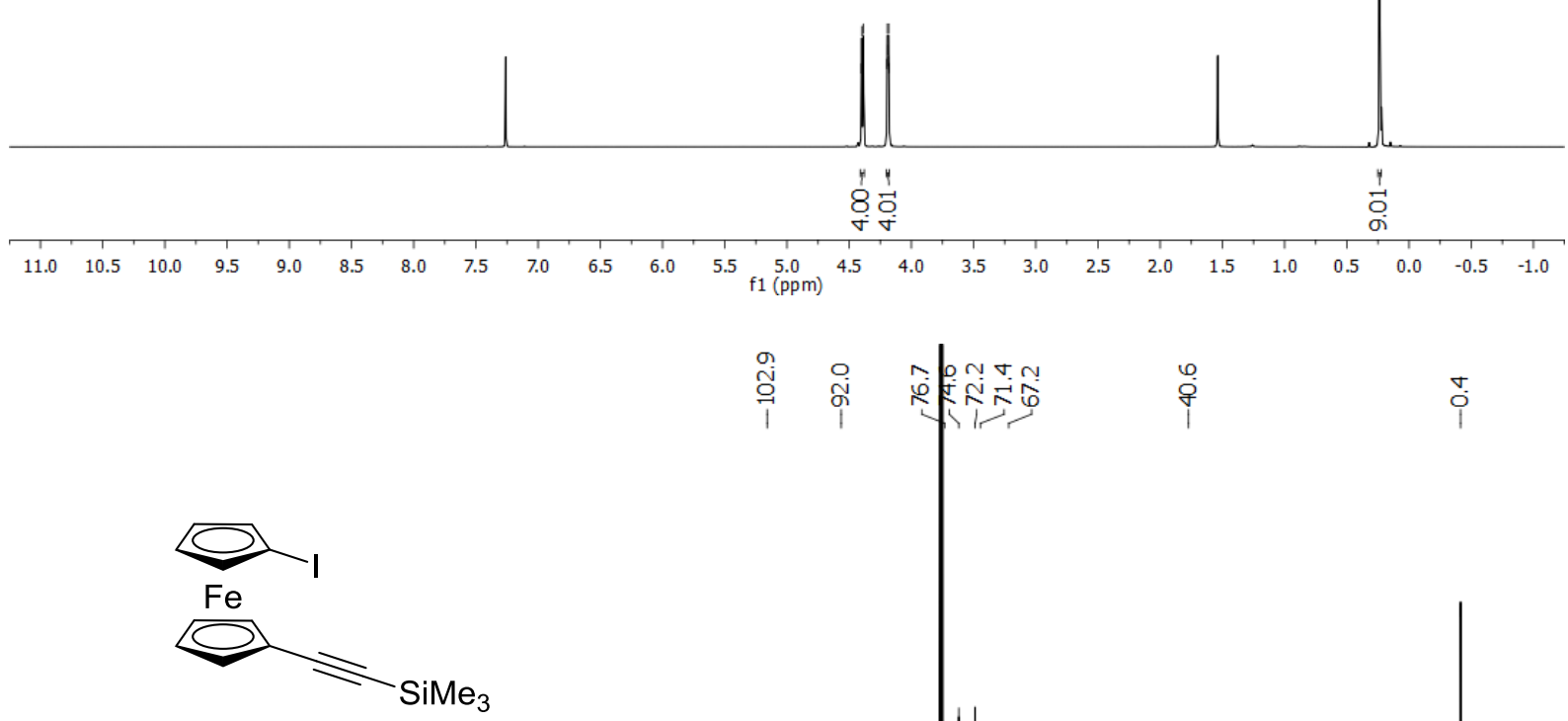

$1 \mathrm{i}$

${ }^{13} \mathrm{C}\left\{{ }^{1} \mathrm{H}\right\}$ NMR (176 $\mathrm{MHz}, \mathrm{CDCl}_{3}$ )

家

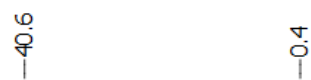

$i_{0}^{1}$ 


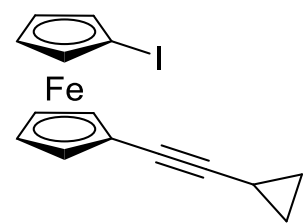

$1 \mathrm{j}$

${ }^{1} \mathrm{H} \mathrm{NMR}\left(500 \mathrm{MHz}, \mathrm{CDCl}_{3}\right)$

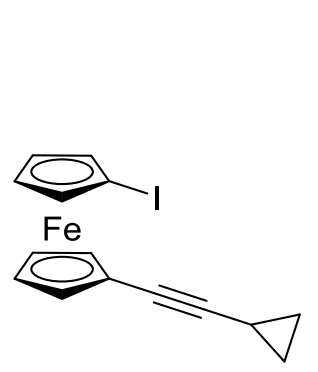

$1 \mathrm{j}$

${ }^{13} \mathrm{C}\left\{{ }^{1} \mathrm{H}\right\}$ NMR $\left(126 \mathrm{MHz}, \mathrm{CDCl}_{3}\right)$

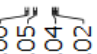

尝

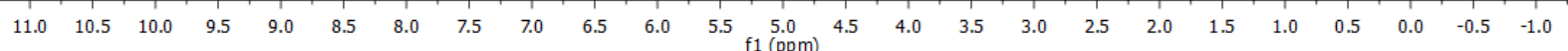

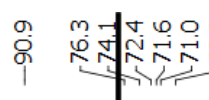

$\stackrel{\overrightarrow{+}}{\overrightarrow{+}}$

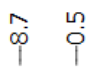

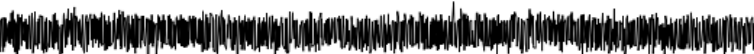

$\begin{array}{lllllllllllllllllllllll}210 & 200 & 190 & 180 & 170 & 160 & 150 & 140 & 130 & 120 & 110 & 100 & 90 & 80 & 70 & 60 & 50 & 40 & 30 & 20 & 10 & 0 & -10\end{array}$ 


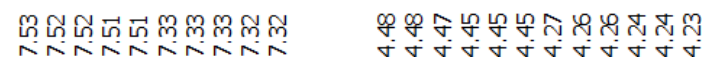

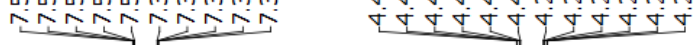

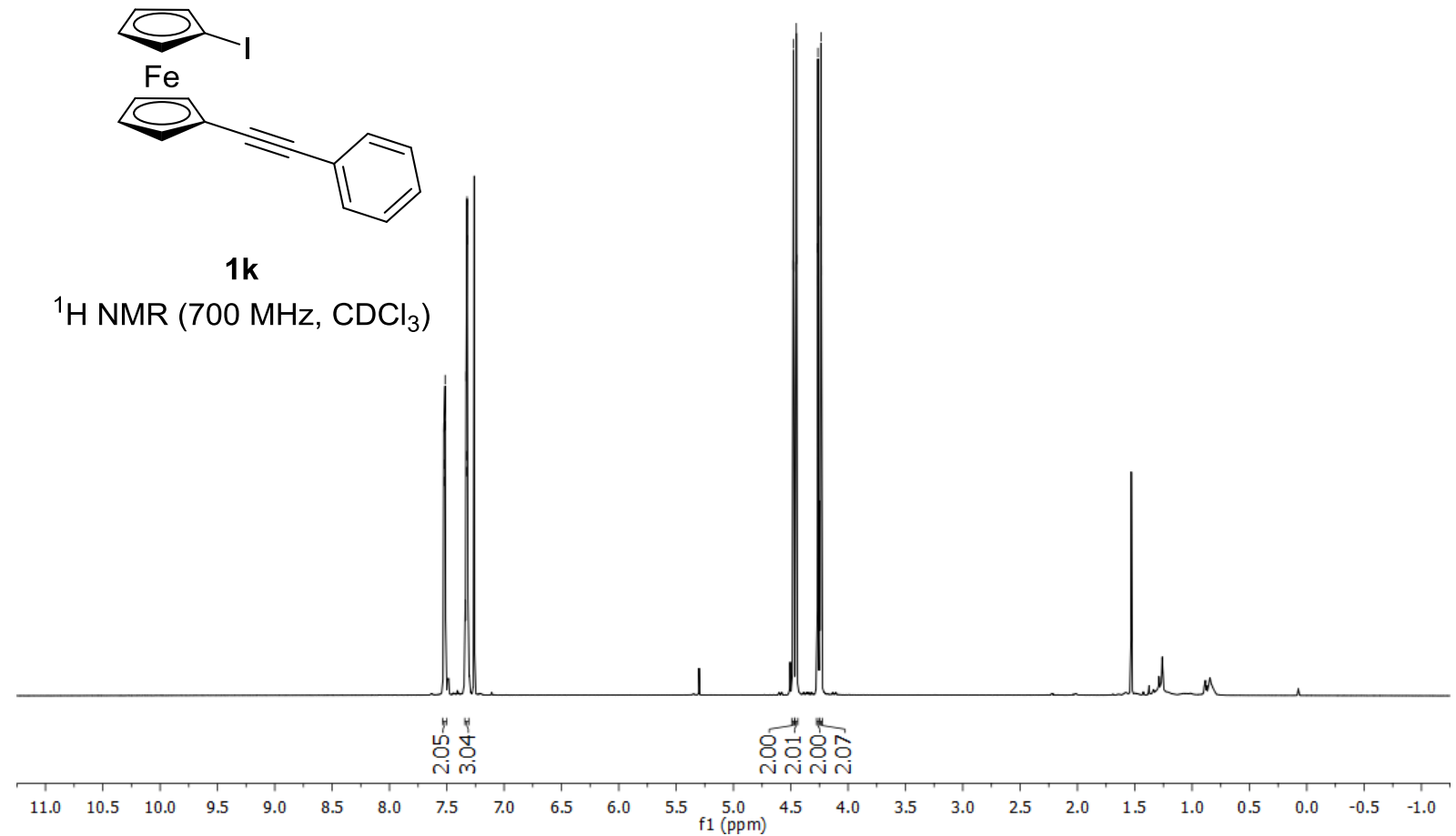

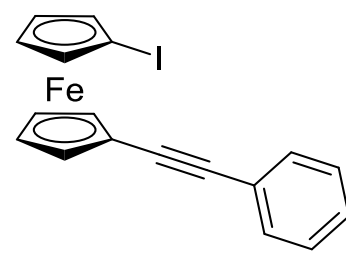

$1 k$

${ }^{13} \mathrm{C}\left\{{ }^{1} \mathrm{H}\right\}$ NMR $\left(176 \mathrm{MHz}, \mathrm{CDCl}_{3}\right)$

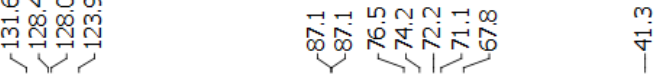

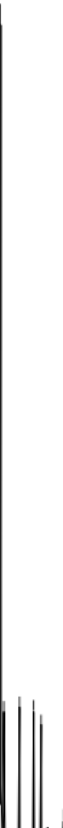

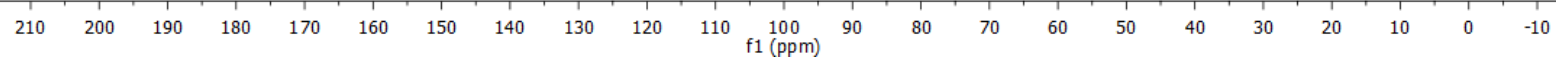




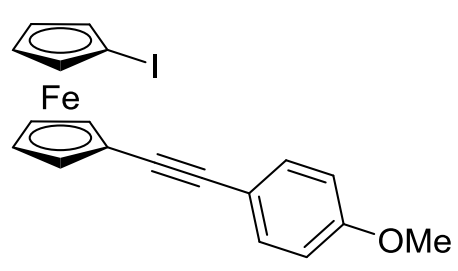

11

${ }^{1} \mathrm{H}$ NMR $\left(700 \mathrm{MHz}, \mathrm{CDCl}_{3}\right)$

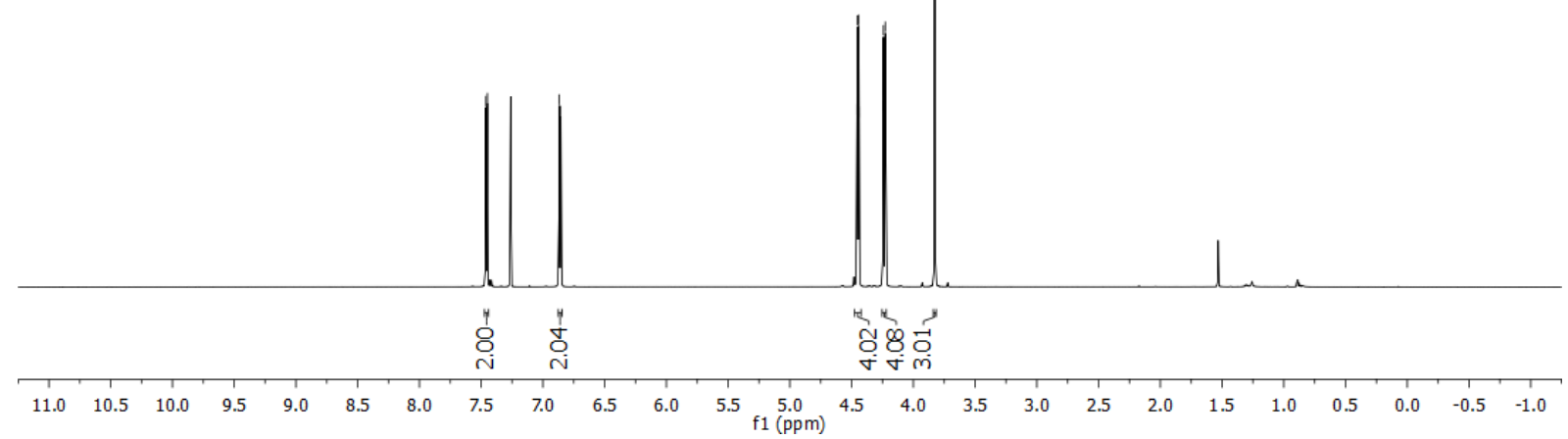

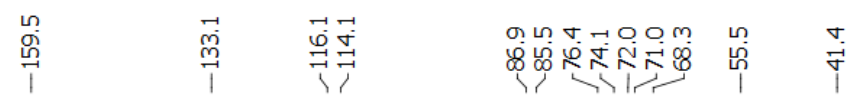

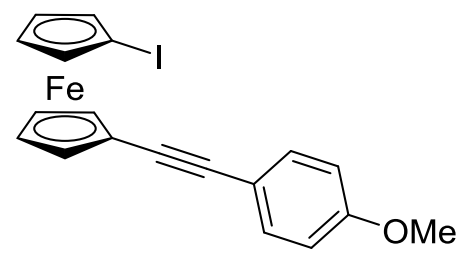

11

${ }^{13} \mathrm{C}\left\{{ }^{1} \mathrm{H}\right\} \operatorname{NMR}\left(176 \mathrm{MHz}, \mathrm{CDCl}_{3}\right)$

$\begin{array}{llllllllllll}210 & 200 & 190 & 180 & 170 & 160 & 150 & 140 & 130 & 120 & 110 & 100 \\ \mathrm{f} 1(\mathrm{ppm}) & 90\end{array}$ 

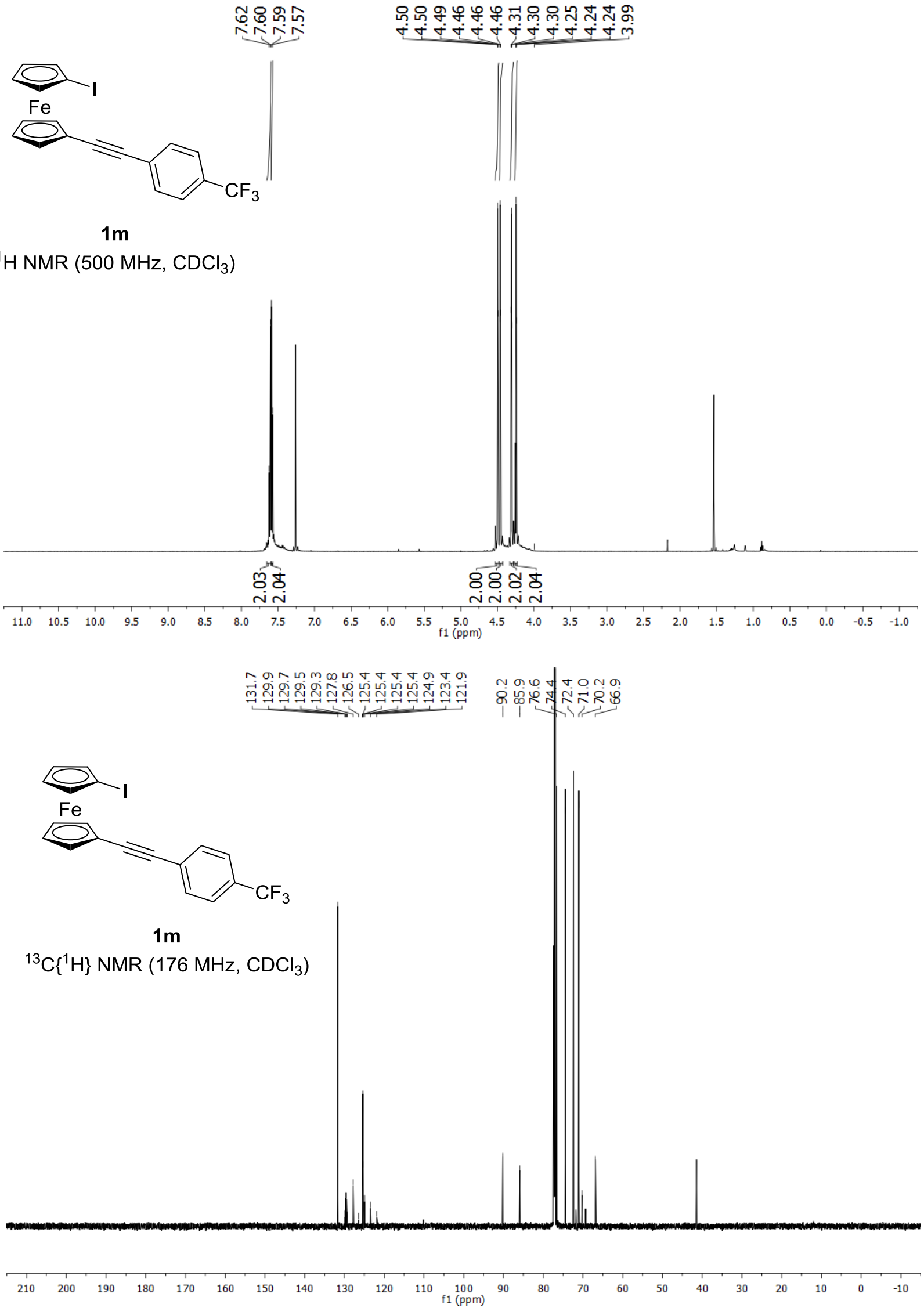


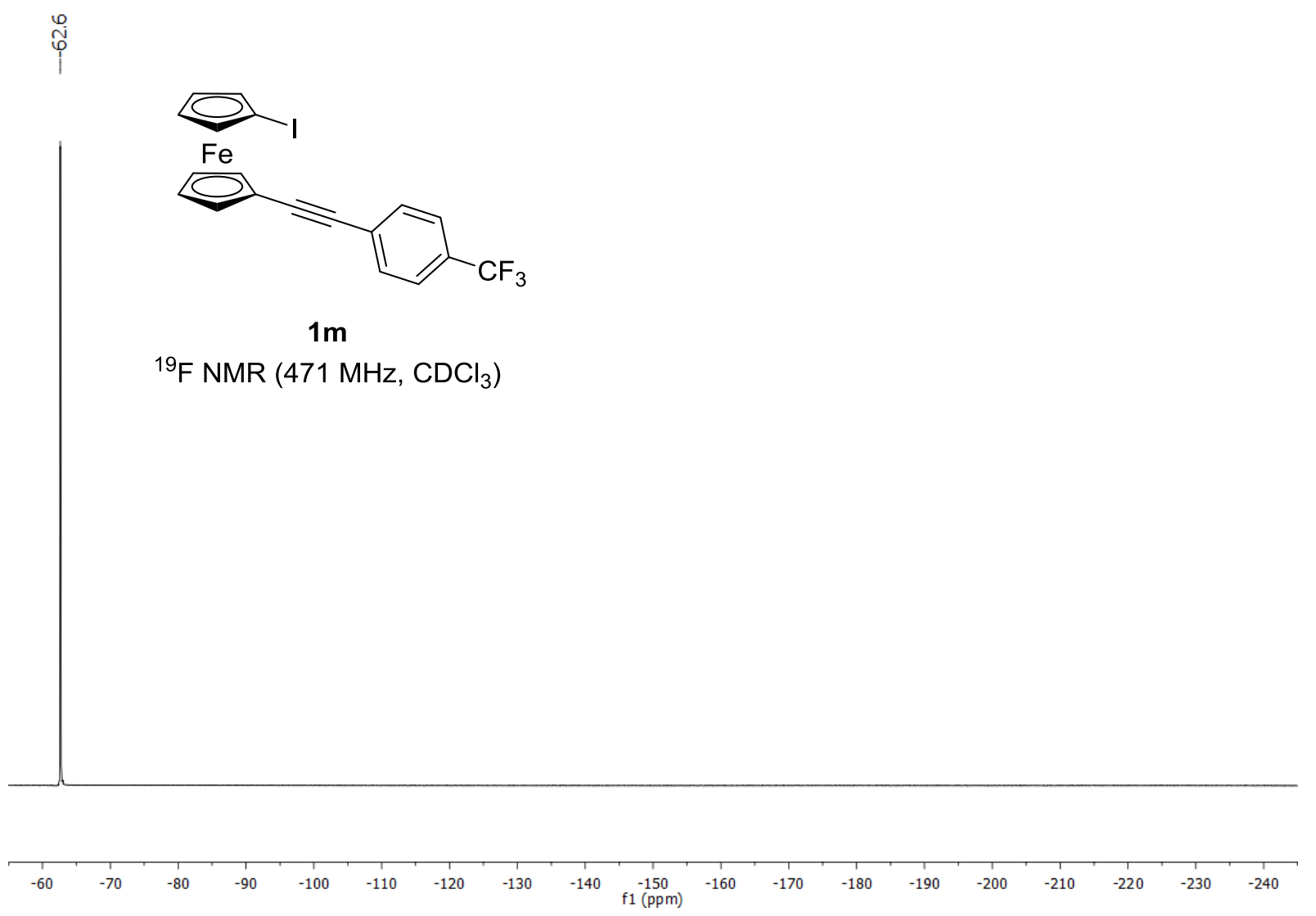




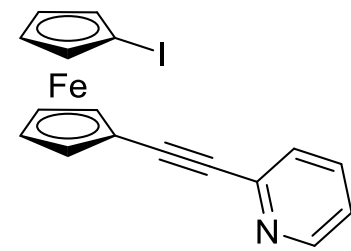

1n

${ }^{1} \mathrm{H}$ NMR $\left(500 \mathrm{MHz}, \mathrm{CDCl}_{3}\right)$

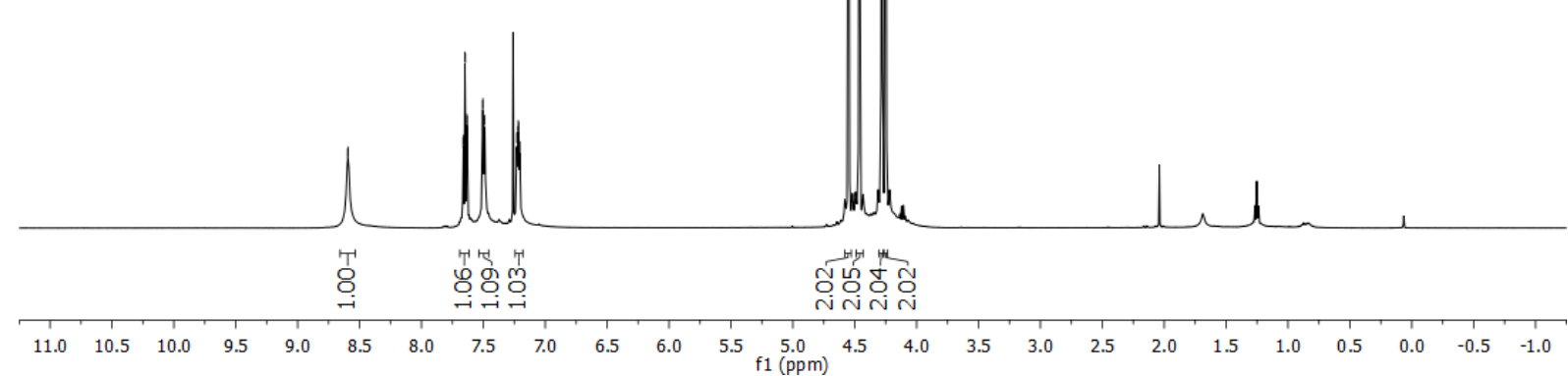

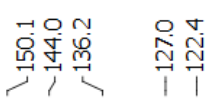

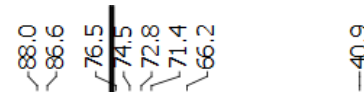

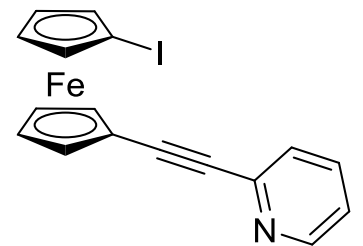

$1 \mathrm{n}$

${ }^{13} \mathrm{C}\left\{{ }^{1} \mathrm{H}\right\}$ NMR $\left(126 \mathrm{MHz}, \mathrm{CDCl}_{3}\right)$

$\begin{array}{lllllllllll}210 & 200 & 190 & 180 & 170 & 160 & 150 & 140 & 130 & 120 & 110 \\ \mathrm{f} 1(\mathrm{ppm}) & 100\end{array}$ 


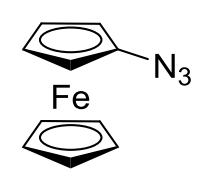

$2 a$

${ }^{1} \mathrm{H}$ NMR (400 MHz, $\mathrm{CDCl}_{3}$ )
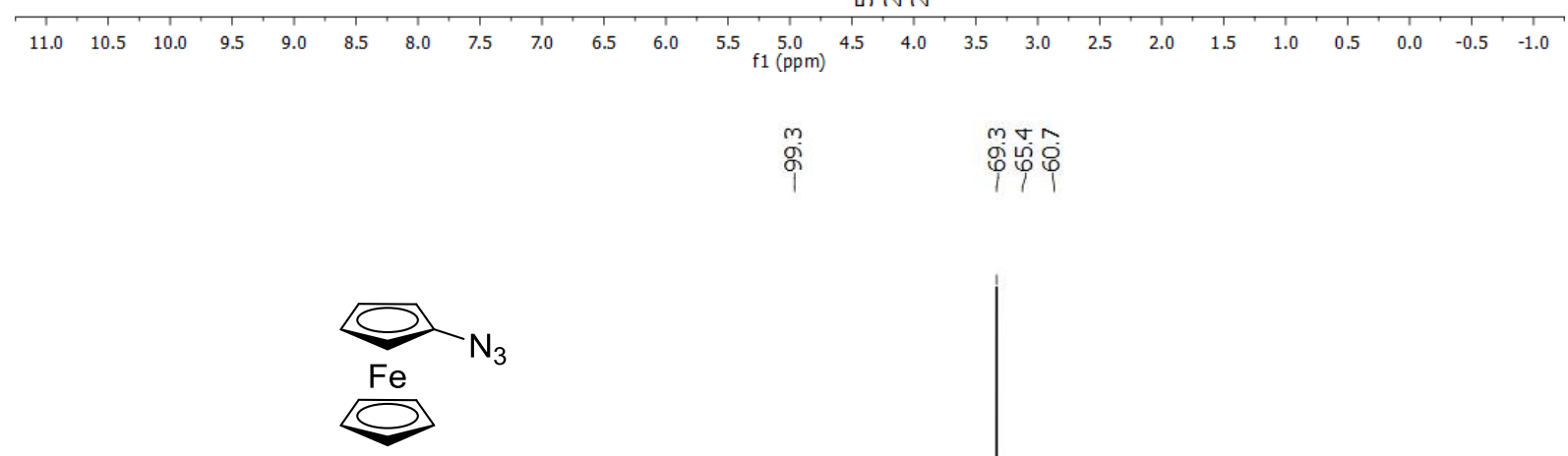

2a

${ }^{13} \mathrm{C}\left\{{ }^{1} \mathrm{H}\right\}$ NMR $\left(100 \mathrm{MHz}, \mathrm{CDCl}_{3}\right)$

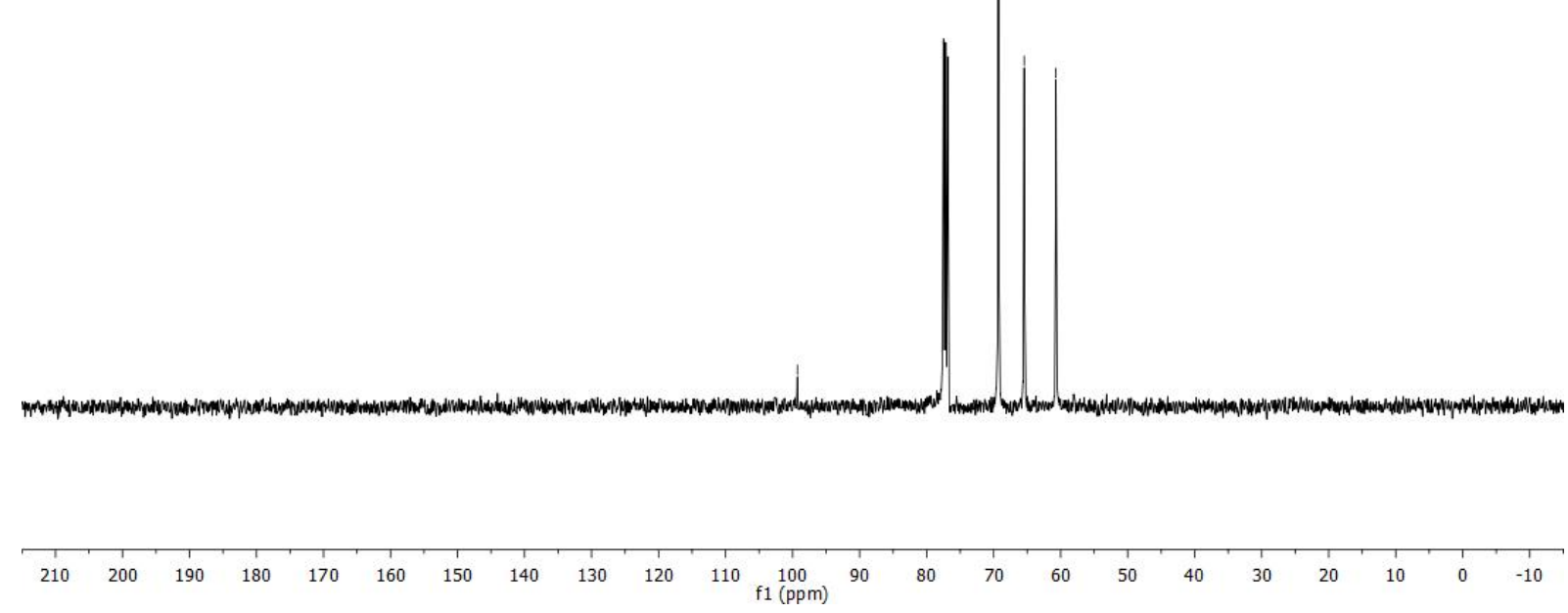




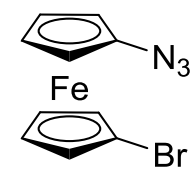

2b

${ }^{1} \mathrm{H}$ NMR $\left(500 \mathrm{MHz}, \mathrm{CDCl}_{3}\right)$

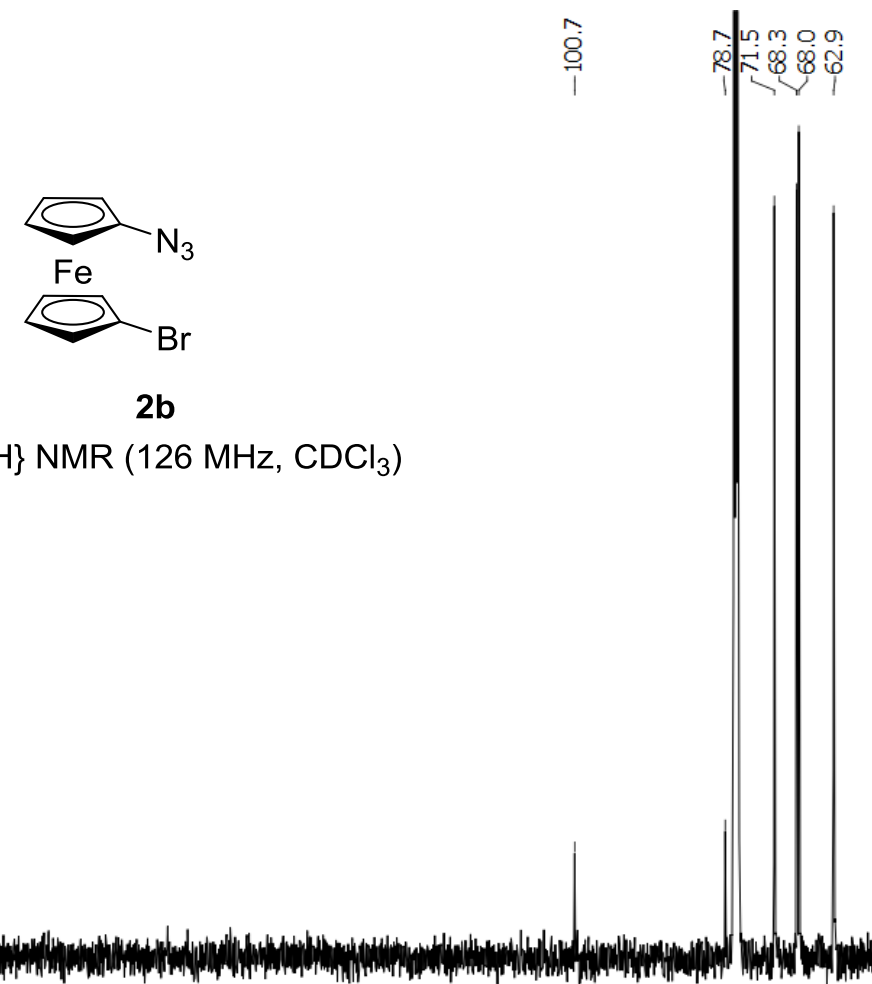

2b

${ }^{13} \mathrm{C}\left\{{ }^{1} \mathrm{H}\right\}$ NMR $\left(126 \mathrm{MHz}, \mathrm{CDCl}_{3}\right)$

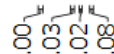

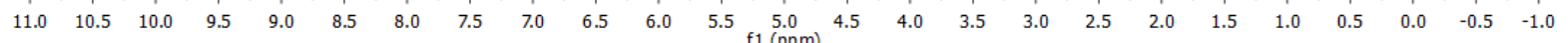

(N)

$\begin{array}{lllllllllllllllllllllllll} & 210 & 200 & 190 & 180 & 170 & 160 & 150 & 140 & 130 & 120 & 110 & 100 & 90 & 80 & 70 & 60 & 50 & 40 & 30 & 20 & 10 & 0 & -10\end{array}$ 


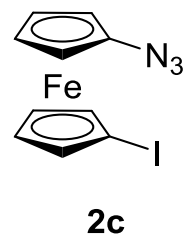

${ }^{1} \mathrm{H}$ NMR $\left(400 \mathrm{MHz}, \mathrm{CDCl}_{3}\right.$ )

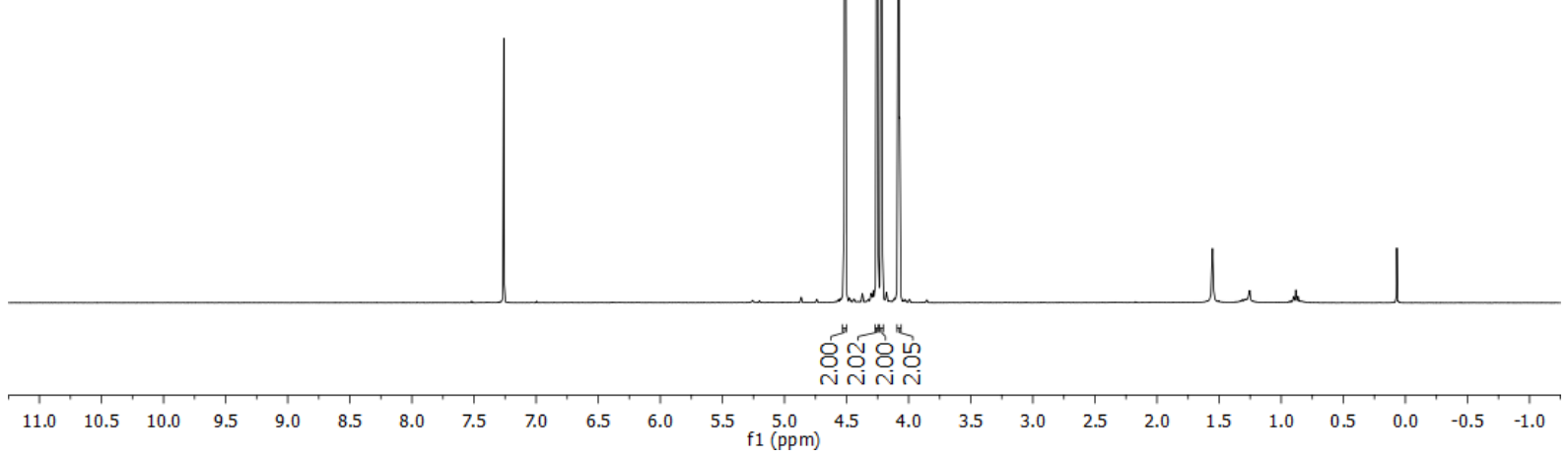

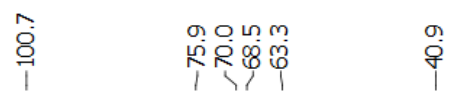
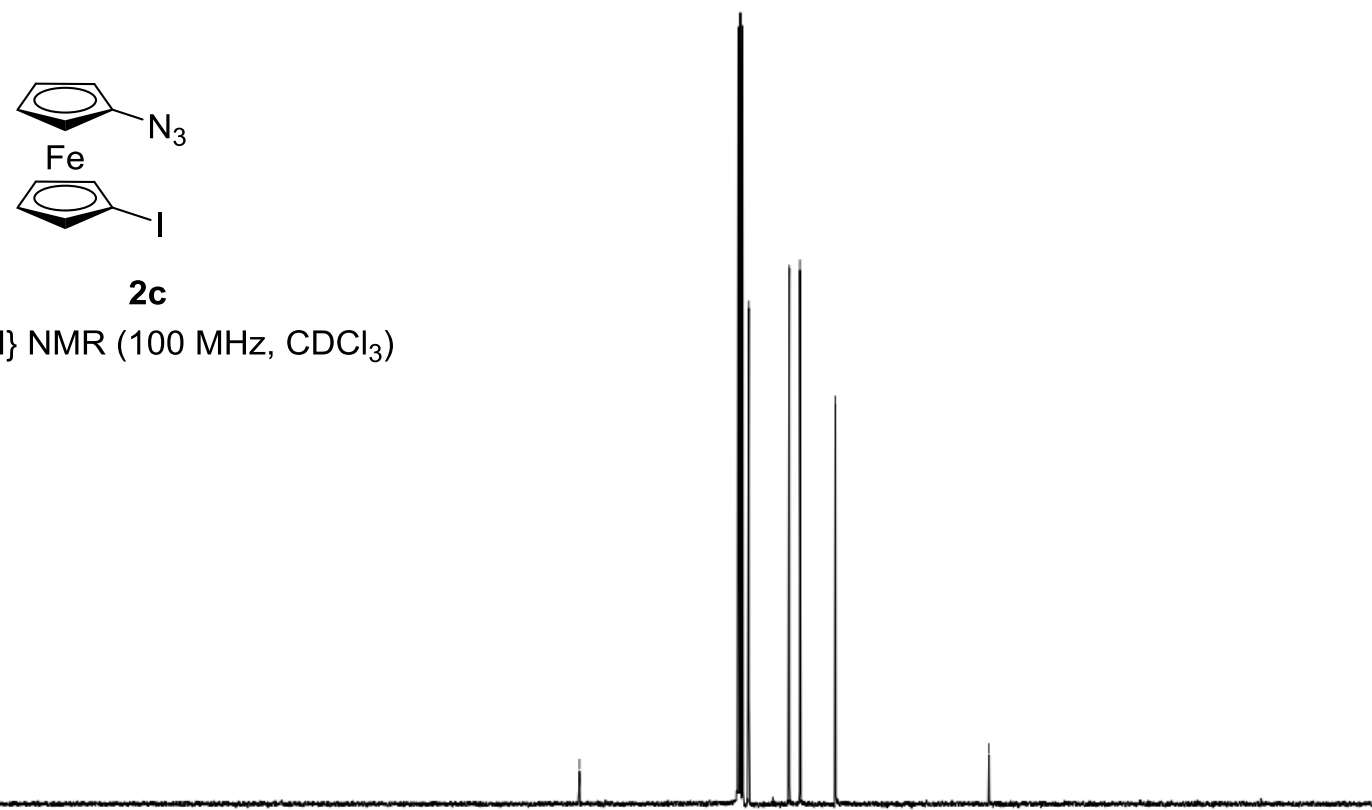

210

$\begin{array}{llllll}200 & 190 & 180 & 170 & 160 & 1\end{array}$

$\begin{array}{lll}140 & 130 \quad 120\end{array}$

$10 \stackrel{100}{100} 90$

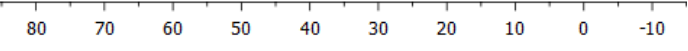




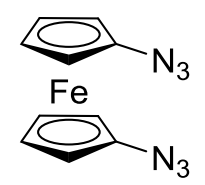

2d

${ }^{1} \mathrm{H}$ NMR $\left(700 \mathrm{MHz}, \mathrm{CDCl}_{3}\right)$
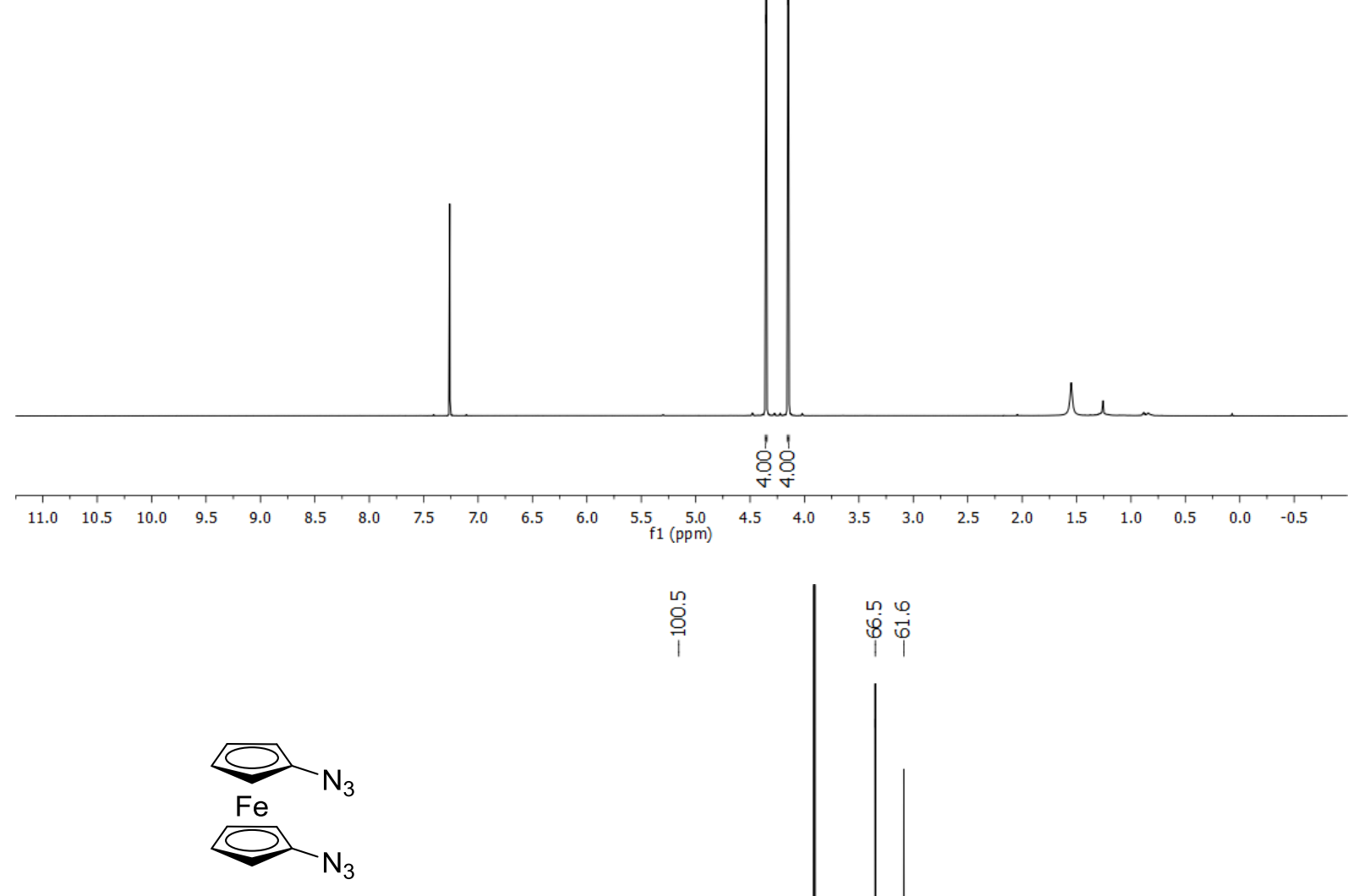

2d

${ }^{13} \mathrm{C}\left\{{ }^{1} \mathrm{H}\right\}$ NMR (176 MHz, $\mathrm{CDCl}_{3}$ )

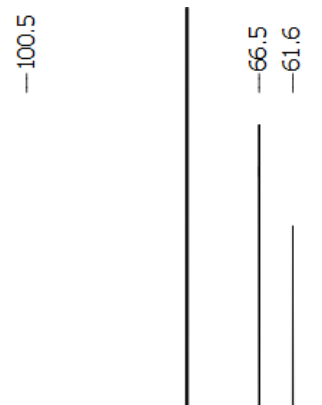

$\begin{array}{lllllllllllllllllllllll}210 & 200 & 190 & 180 & 170 & 160 & 150 & 140 & 130 & 120 & 110 & 100 & 90 & 80 & 70 & 60 & 50 & 40 & 30 & 20 & 10 & 0 & -10\end{array}$ 


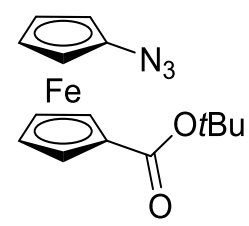

$2 e$

${ }^{1} \mathrm{H}$ NMR $\left(400 \mathrm{MHz}, \mathrm{CDCl}_{3}\right)$

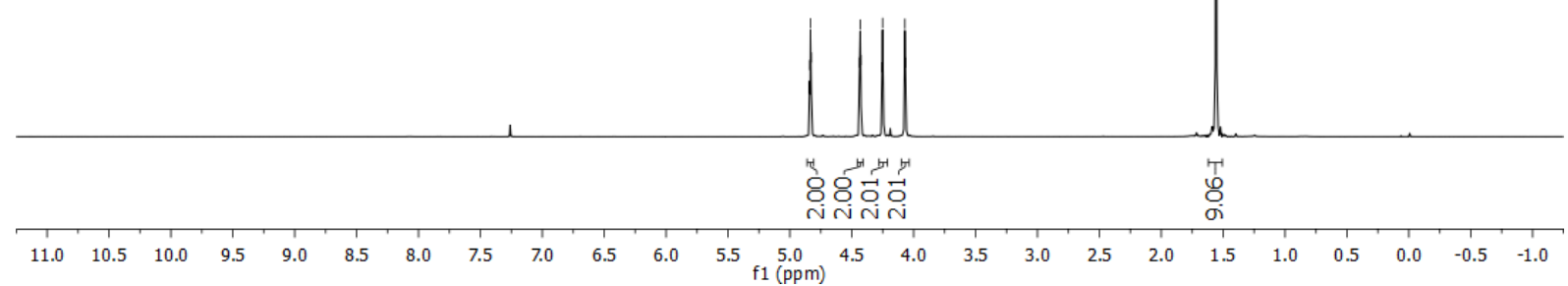

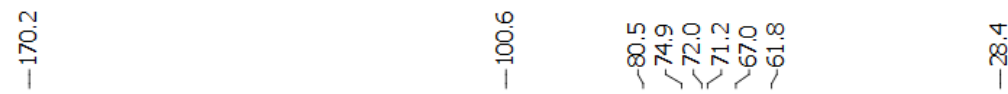

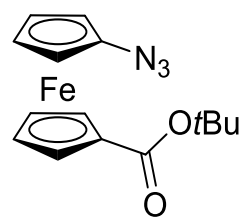

$2 e$

${ }^{13} \mathrm{C}\left\{{ }^{1} \mathrm{H}\right\}$ NMR $\left(100 \mathrm{MHz}, \mathrm{CDCl}_{3}\right)$

210

200

$\begin{array}{lllllll}180 & 170 & 160 & 150 & 140 & 130 & 120\end{array}$

$110 \frac{100}{f 1(p p m)}$ 


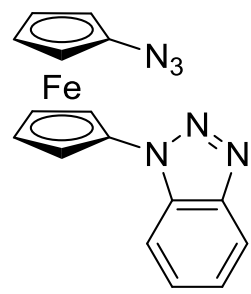

$2 f$

${ }^{1} \mathrm{H} \mathrm{NMR}\left(400 \mathrm{MHz}, \mathrm{CDCl}_{3}\right)$

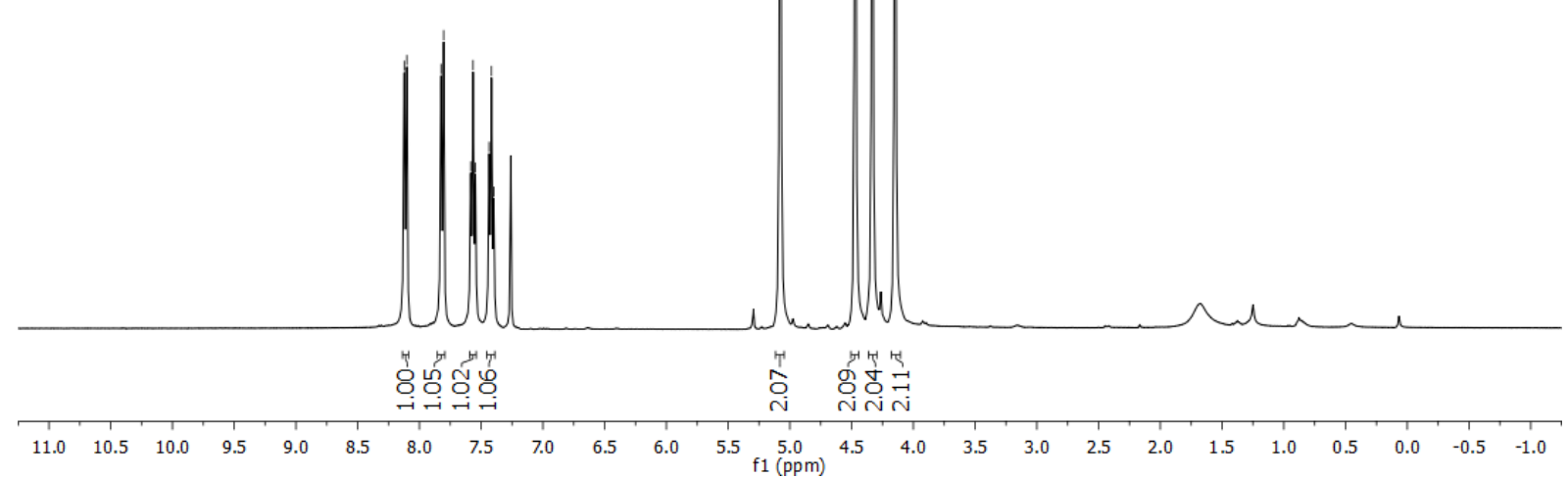

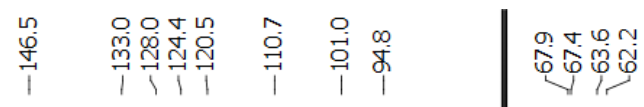

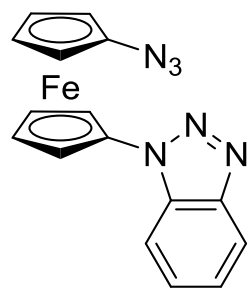

$2 f$

${ }^{13} \mathrm{C}\left\{{ }^{1} \mathrm{H}\right\}$ NMR $\left(100 \mathrm{MHz}, \mathrm{CDCl}_{3}\right)$ 


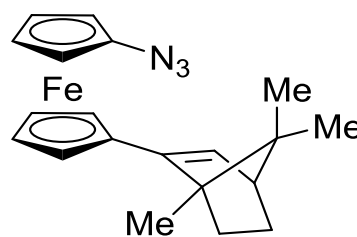

2g

${ }^{1} \mathrm{H}$ NMR $\left(400 \mathrm{MHz}, \mathrm{CDCl}_{3}\right)$

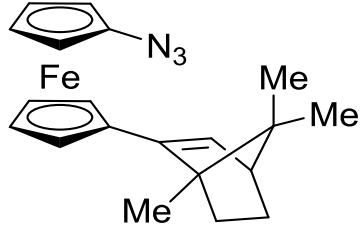

2g

${ }^{13} \mathrm{C}\left\{{ }^{1} \mathrm{H}\right\} \mathrm{NMR}\left(100 \mathrm{MHz}, \mathrm{CDCl}_{3}\right)$

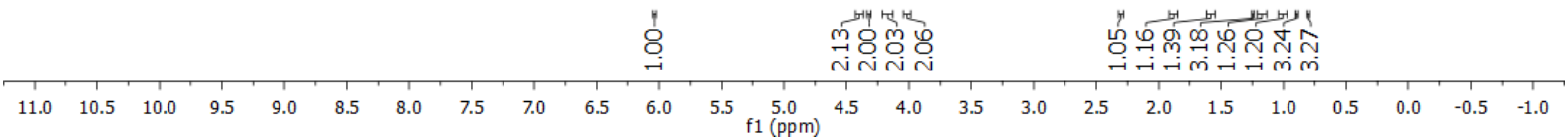




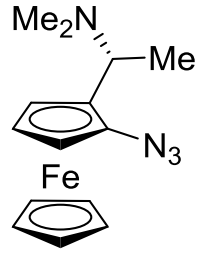

2h

${ }^{1} \mathrm{H}$ NMR $\left(600 \mathrm{MHz}, \mathrm{CDCl}_{3}\right)$
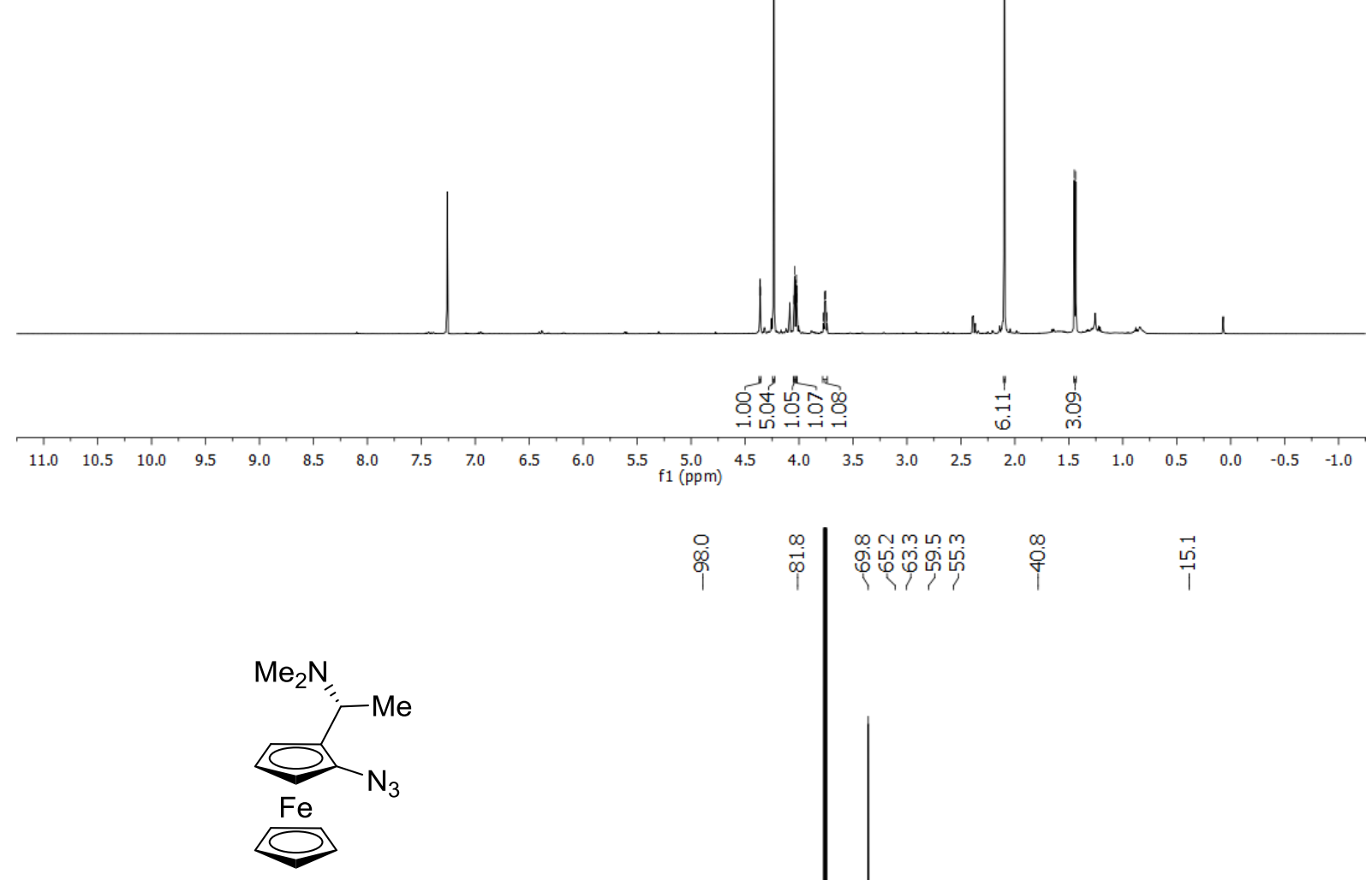

2h

$\left.{ }^{13} \mathrm{C}\left\{{ }^{1} \mathrm{H}\right\} \operatorname{NMR}(151 \mathrm{MHz}, \mathrm{CDCl})_{3}\right)$

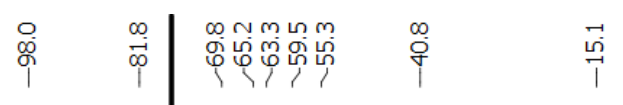

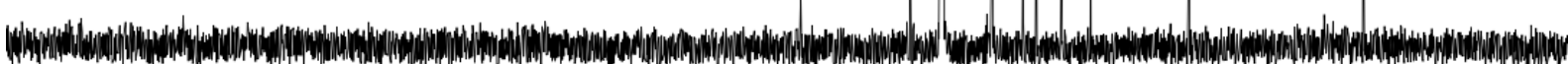

$\begin{array}{lllllllllllllllllllllllll}210 & 200 & 190 & 180 & 170 & 160 & 150 & 140 & 130 & 120 & 110 & 100 & 90 & 80 & 70 & 60 & 50 & 40 & 30 & 20 & 10 & 0 & -10\end{array}$ 


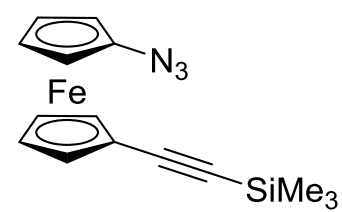

$2 \mathbf{i}$

${ }^{1} \mathrm{H}$ NMR (400 MHz, $\mathrm{CDCl}_{3}$ )

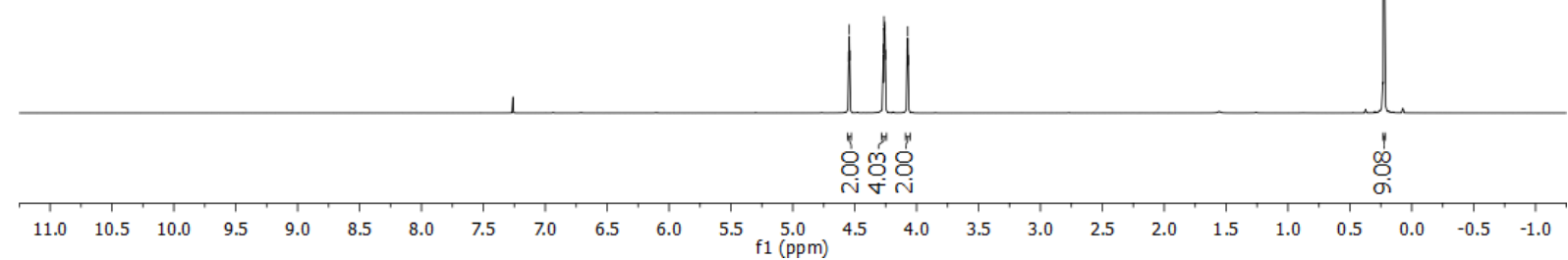

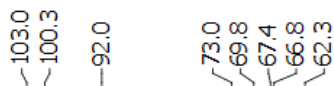

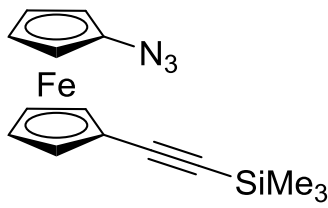

2i

${ }^{13} \mathrm{C}\left\{{ }^{1} \mathrm{H}\right\}$ NMR $\left(100 \mathrm{MHz}, \mathrm{CDCl}_{3}\right)$

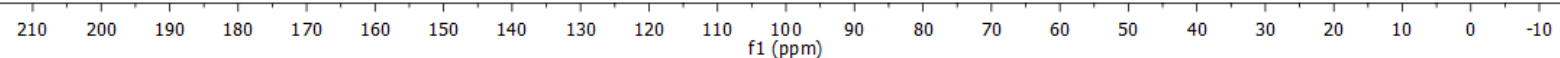




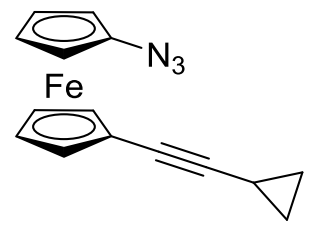

2j

${ }^{1} \mathrm{H}$ NMR $\left(700 \mathrm{MHz}, \mathrm{CDCl}_{3}\right)$
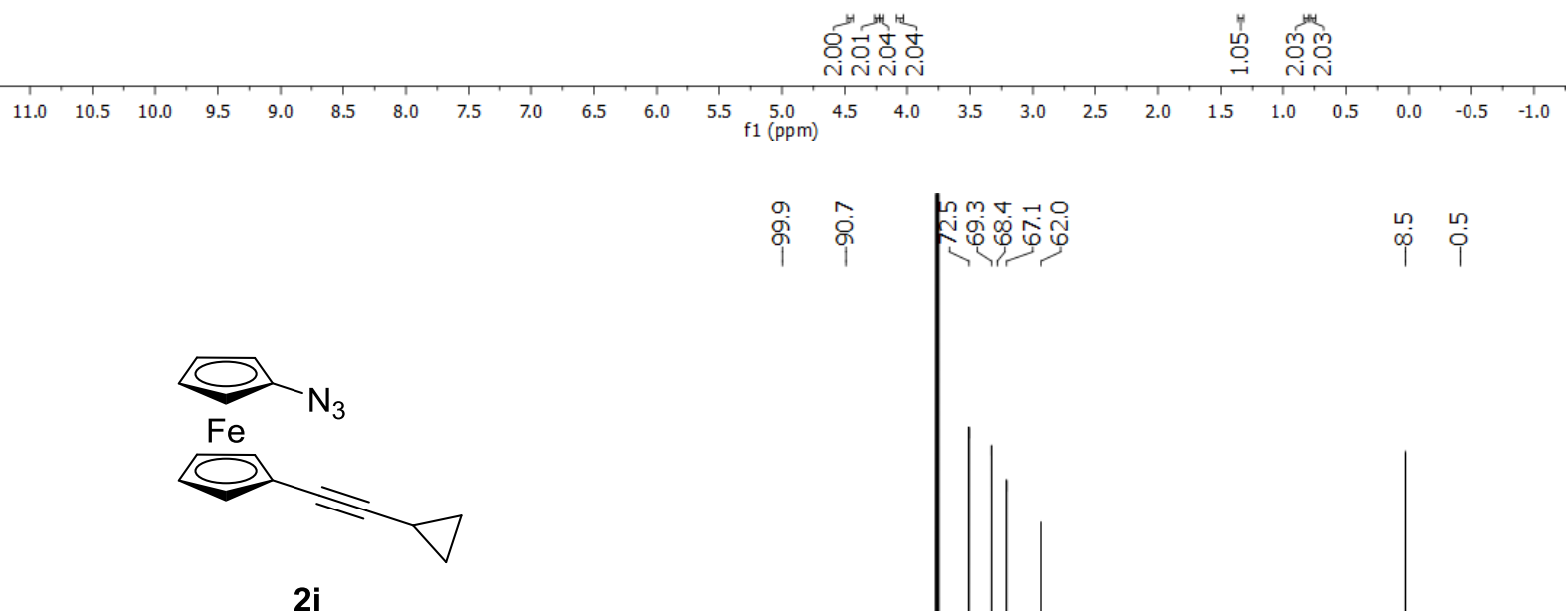

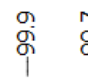

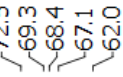

$\stackrel{\text { Ln }}{\infty} \stackrel{\text { Ln }}{i}$

${ }^{13} \mathrm{C}\left\{{ }^{1} \mathrm{H}\right\}$ NMR $\left(176 \mathrm{MHz}, \mathrm{CDCl}_{3}\right)$

$\begin{array}{lllllllllllllllllllllllllll}210 & 200 & 190 & 180 & 170 & 160 & 150 & 140 & 130 & 120 & 110 & 100 & 90 & 80 & 70 & 60 & 50 & 40 & 30 & 20 & 10 & 0 & -10\end{array}$ 


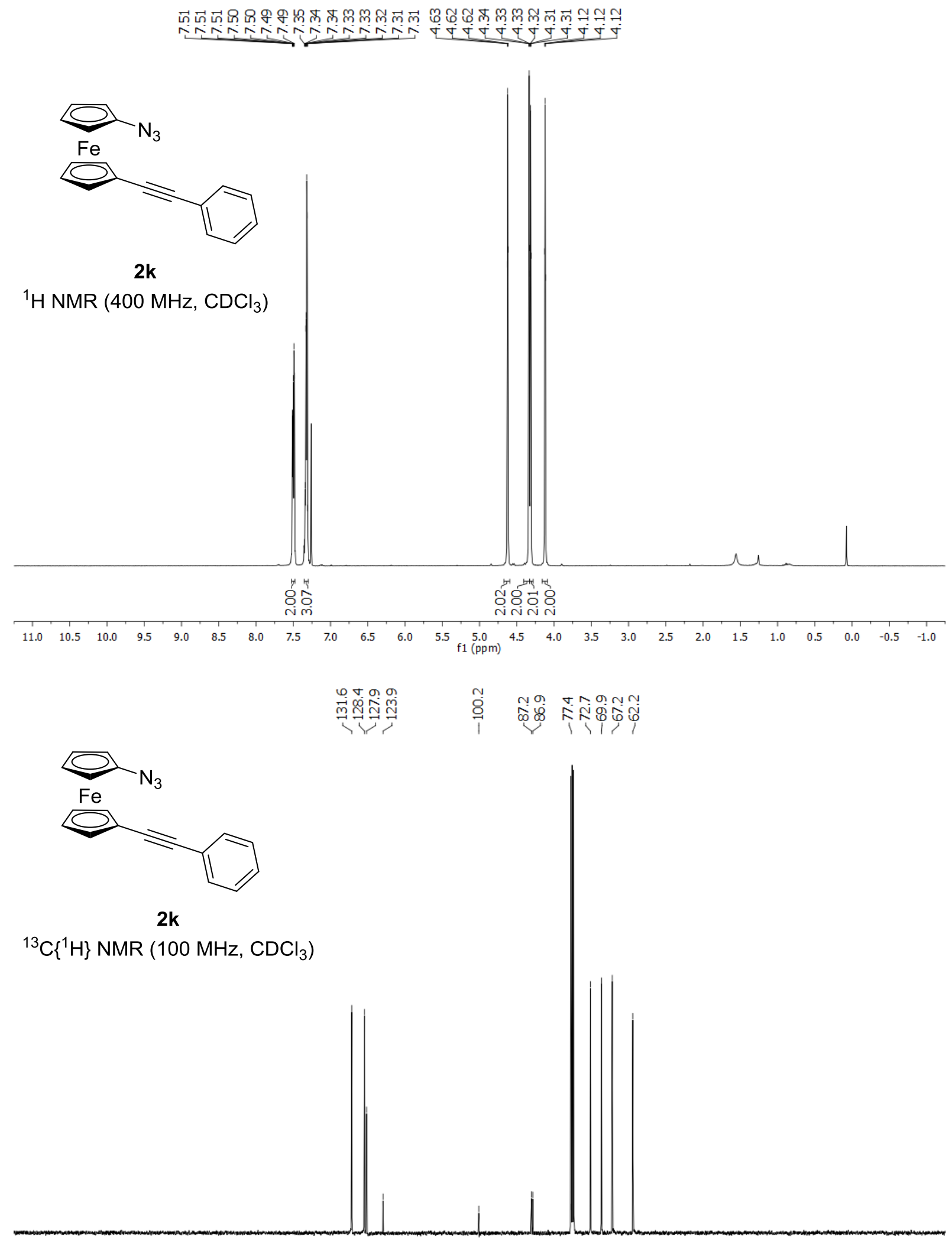

$\begin{array}{lllllllllllllllllllllllll}210 & 200 & 190 & 180 & 170 & 160 & 150 & 140 & 130 & 120 & 110 & \underset{\mathrm{f} 1}{100}(\mathrm{ppm}) & 90 & 80 & 70 & 60 & 50 & 40 & 30 & 20 & 10 & 0 & -10\end{array}$ 


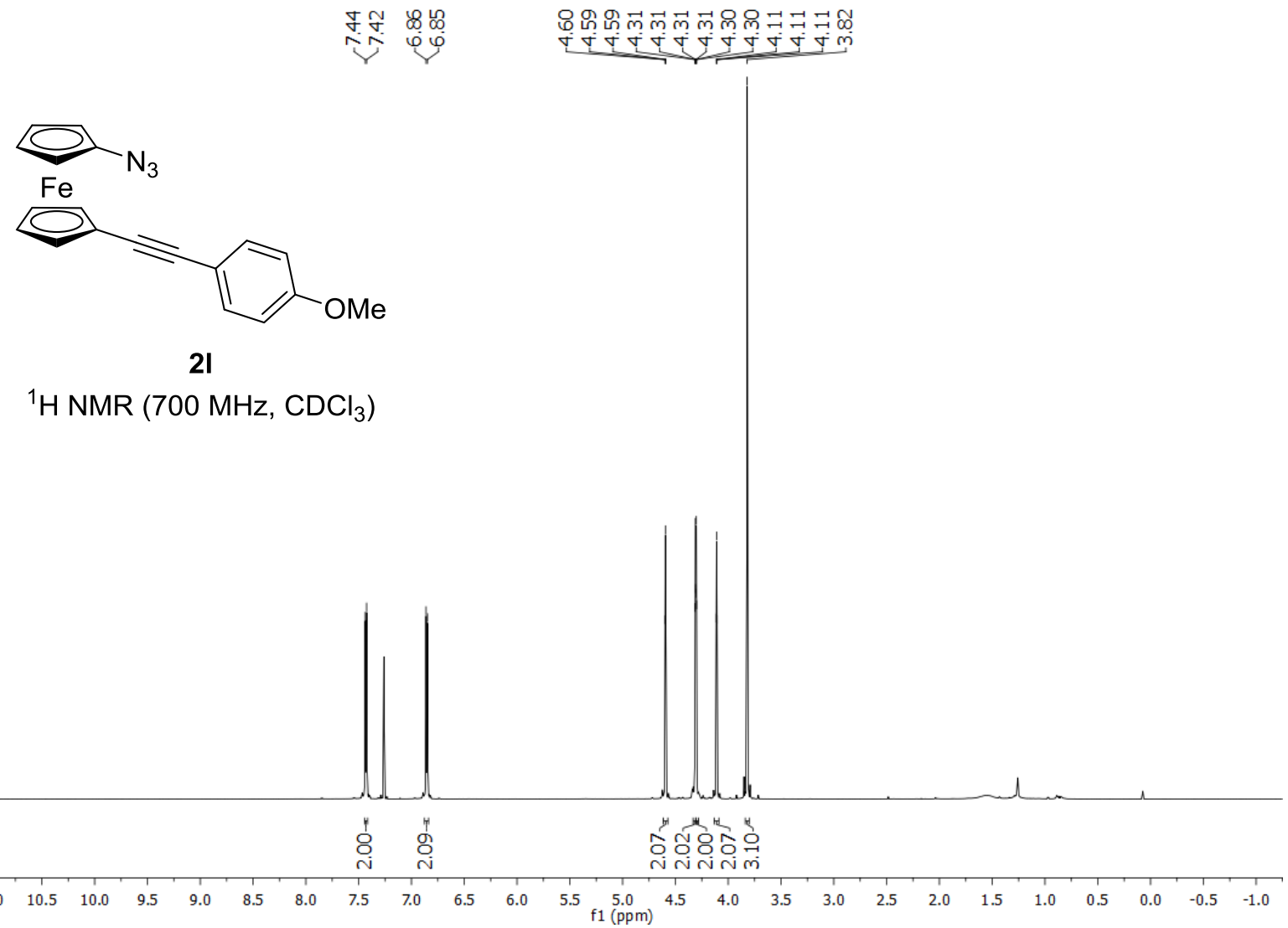

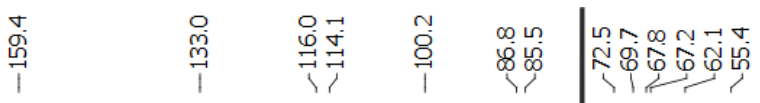

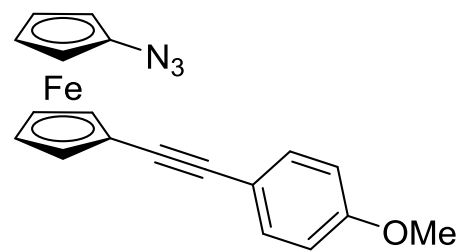

2I

${ }^{13} \mathrm{C}\left\{{ }^{1} \mathrm{H}\right\} \mathrm{NMR}\left(176 \mathrm{MHz}, \mathrm{CDCl}_{3}\right)$

210

$\begin{array}{llllllllllllllllllllllllllll}10 & 200 & 190 & 180 & 170 & 160 & 150 & 140 & 130 & 120 & 110 & 100 & 90 & 80 & 70 & 60 & 50 & 40 & 30 & 20 & 10 & 0 & -10\end{array}$ 


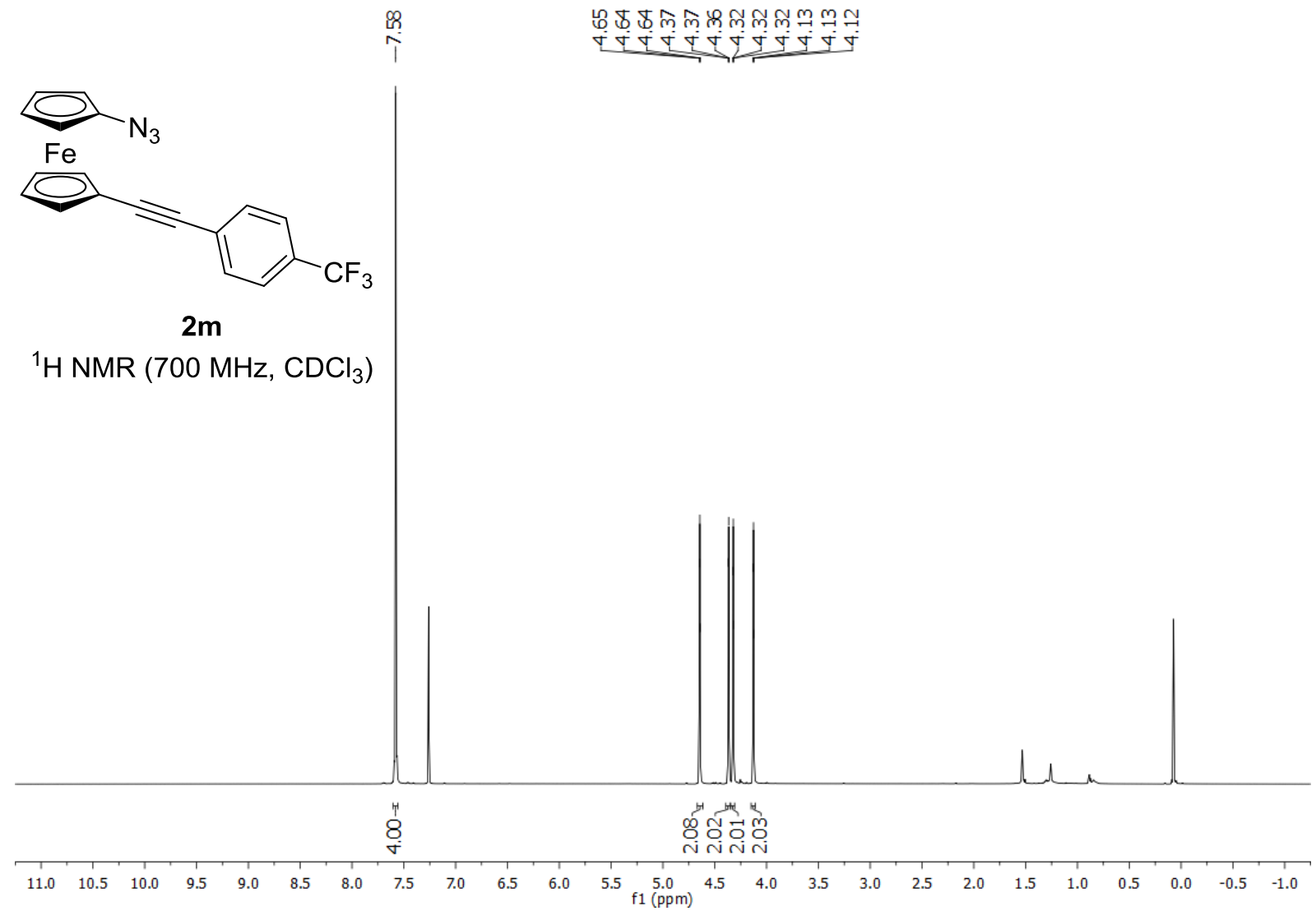

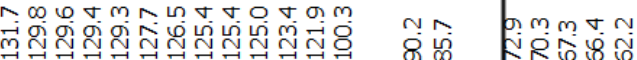

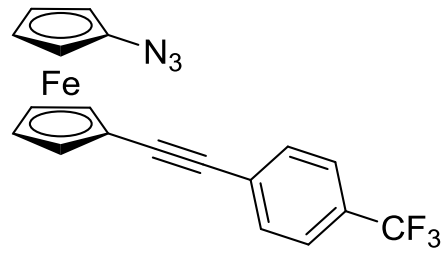

$2 \mathrm{~m}$

${ }^{13} \mathrm{C}\left\{{ }^{1} \mathrm{H}\right\}$ NMR $\left(176 \mathrm{MHz}, \mathrm{CDCl}_{3}\right)$

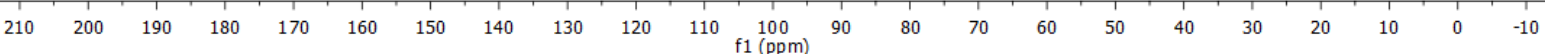




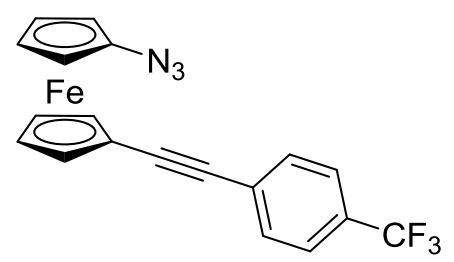

$2 \mathrm{~m}$

${ }^{19} \mathrm{~F} \mathrm{NMR}\left(565 \mathrm{MHz}, \mathrm{CDCl}_{3}\right)$

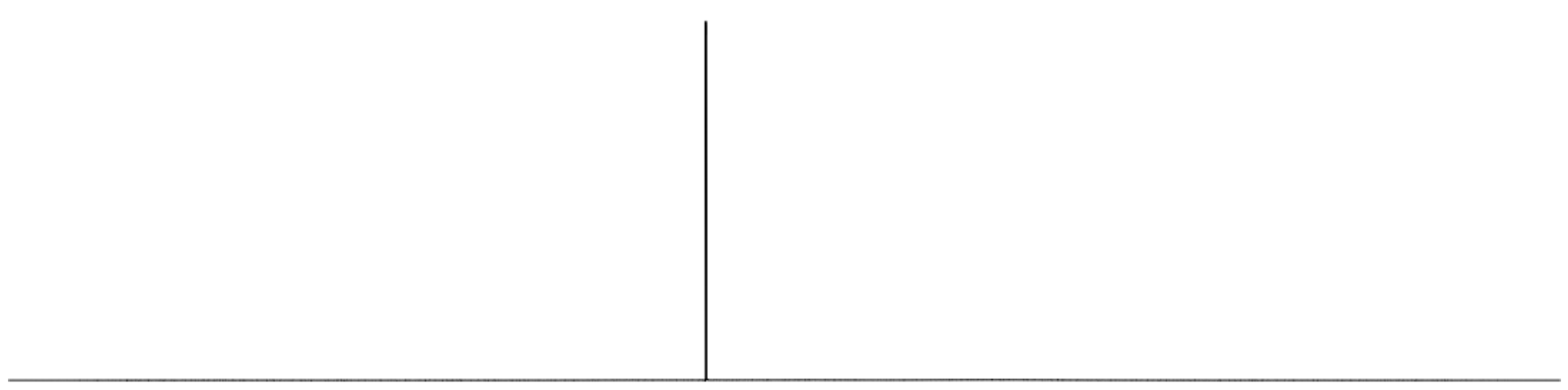

50




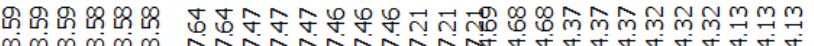

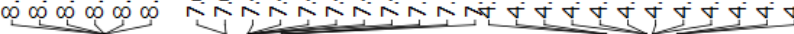

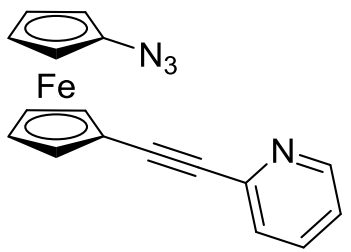

2n

${ }^{1} \mathrm{H}$ NMR $\left(700 \mathrm{MHz}, \mathrm{CDCl}_{3}\right)$

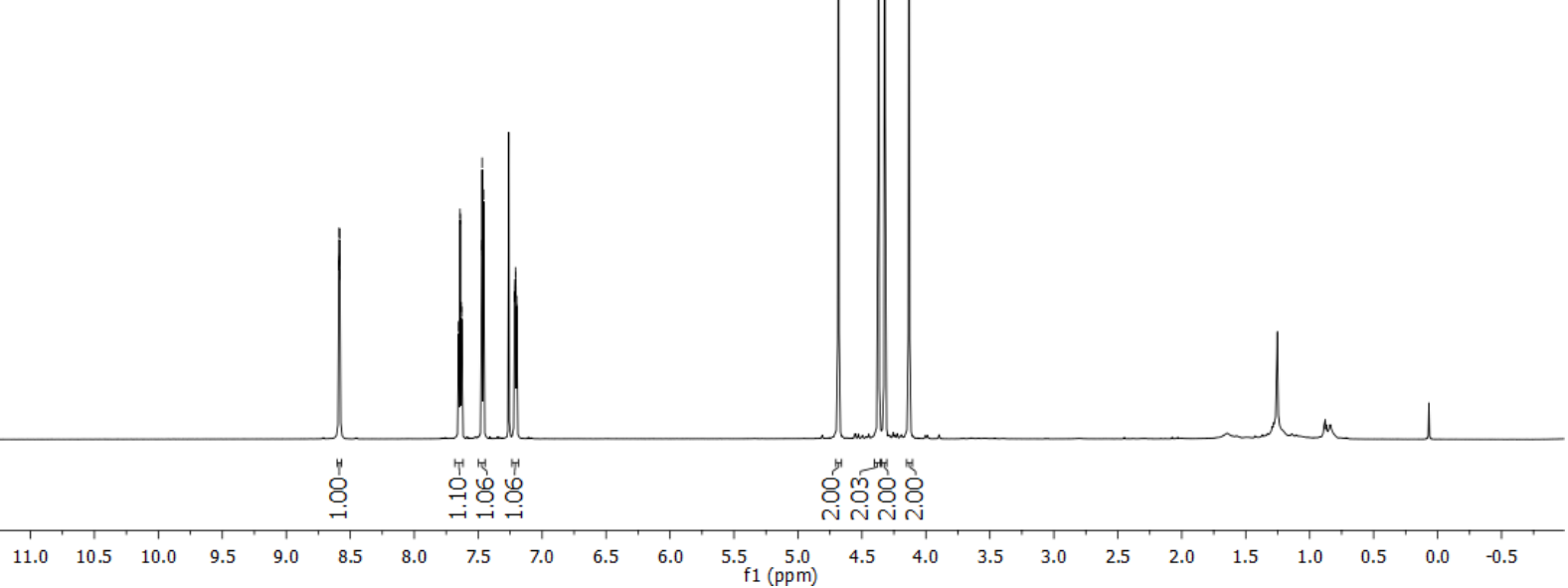

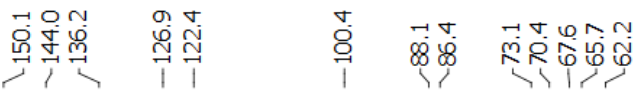

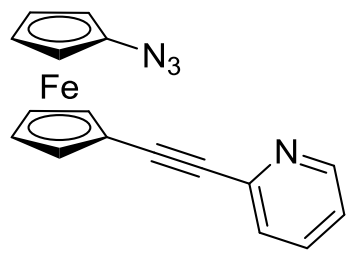

2n

${ }^{13} \mathrm{C}\left\{{ }^{1} \mathrm{H}\right\} \operatorname{NMR}\left(176 \mathrm{MHz}, \mathrm{CDCl}_{3}\right)$

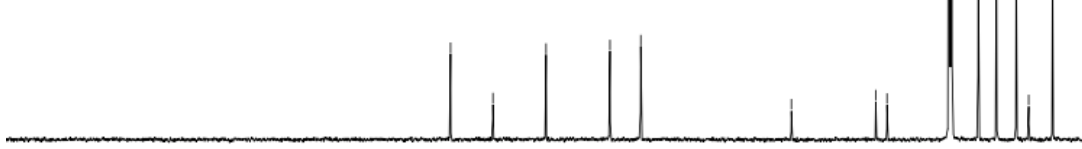

$\begin{array}{lllllllllllllllllllllllll}210 & 200 & 190 & 180 & 170 & 160 & 150 & 140 & 130 & 120 & 110 & 100 & 10 & 80 & 70 & 60 & 50 & 40 & 30 & 20 & 10 & 0 & -10\end{array}$ 

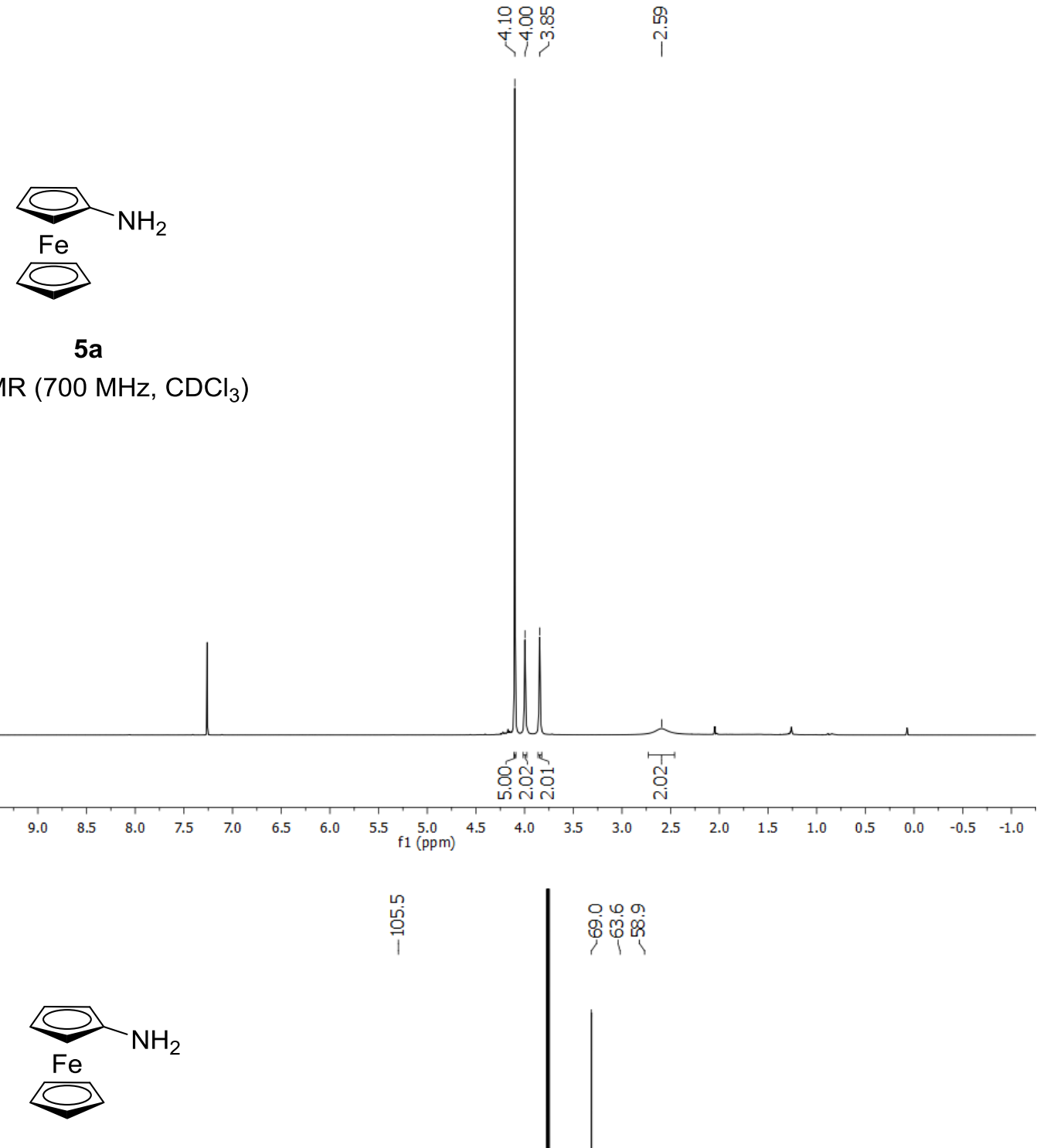

$5 a$

${ }^{13} \mathrm{C}\left\{{ }^{1} \mathrm{H}\right\}$ NMR (176 MHz, $\mathrm{CDCl}_{3}$ )

$\begin{array}{llllllllllllllllllllllllll}210 & 200 & 190 & 180 & 170 & 160 & 150 & 140 & 130 & 120 & 110 & 100 & 90 & 80 & 70 & 60 & 50 & 40 & 30 & 20 & 10 & 0 & -10\end{array}$ 


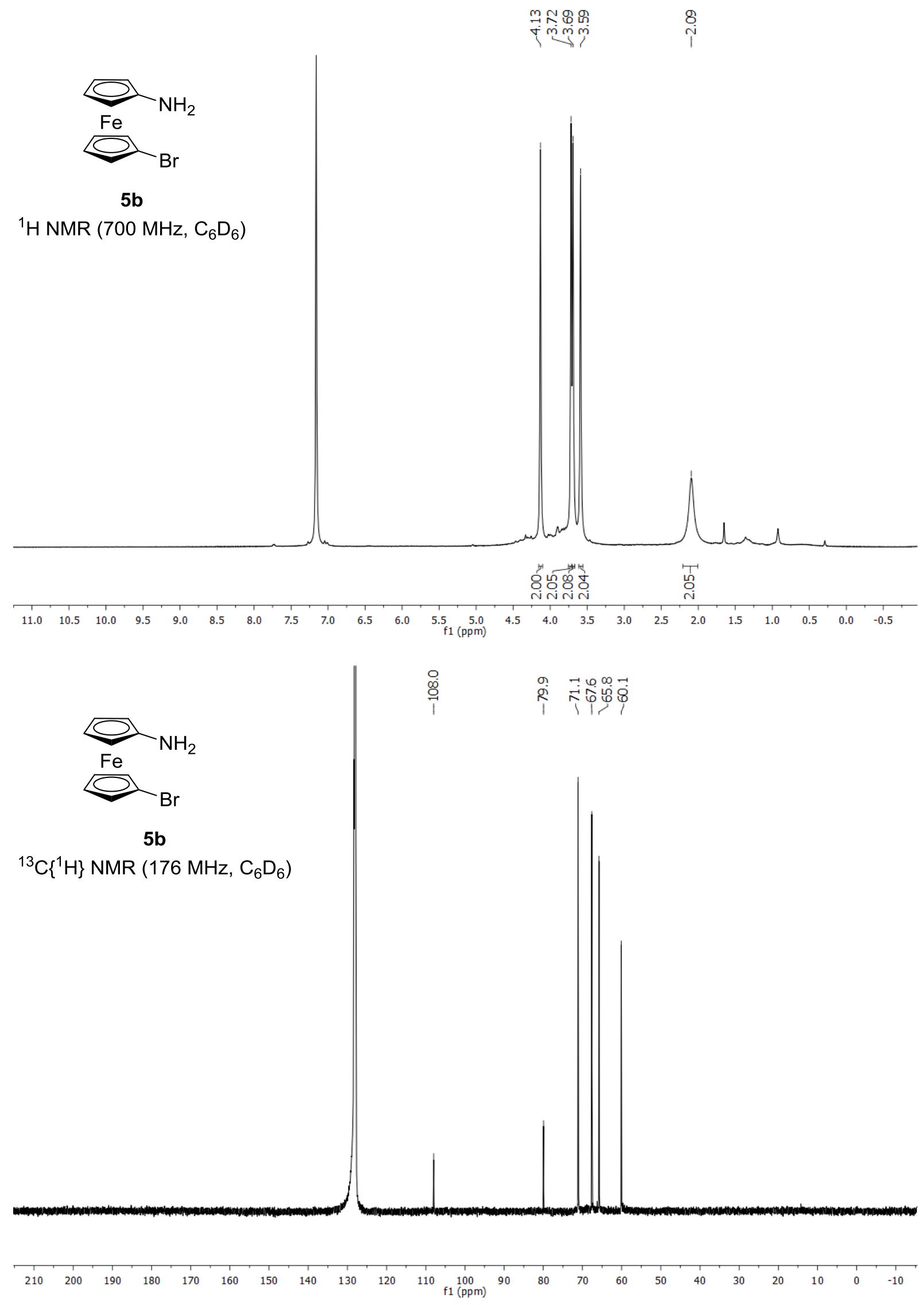




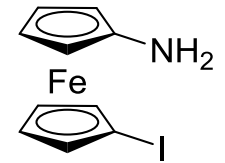

$5 c$

${ }^{1} \mathrm{H}$ NMR $\left(500 \mathrm{MHz}, \mathrm{CDCl}_{3}\right)$

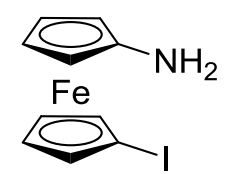

$5 c$

${ }^{13} \mathrm{C}\left\{{ }^{1} \mathrm{H}\right\} \operatorname{NMR}\left(126 \mathrm{MHz}, \mathrm{CDCl}_{3}\right)$

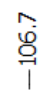
Wh

${ }_{f 1(\mathrm{ppm})}^{5.0} 4$.

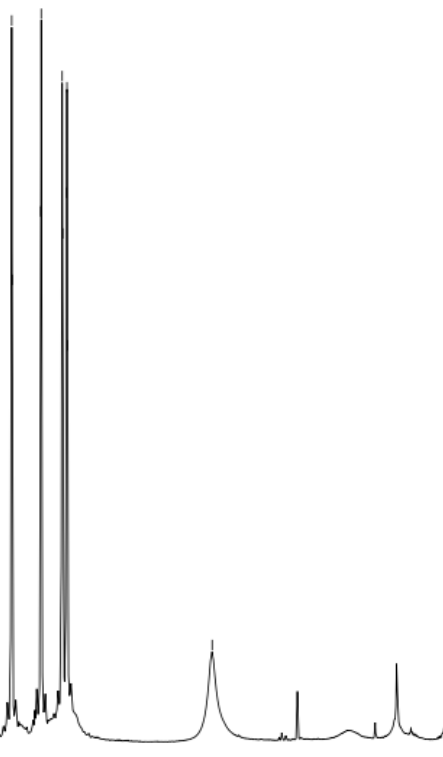

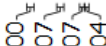

ininis

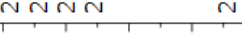

\section{$\overbrace{2.5}^{1}$}

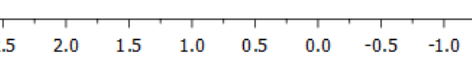

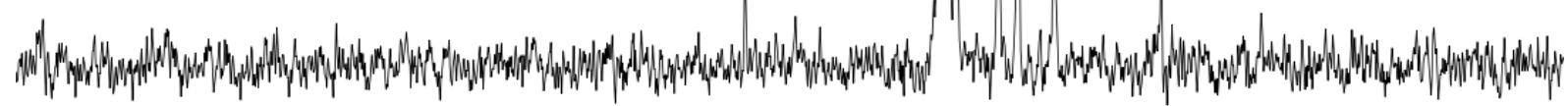

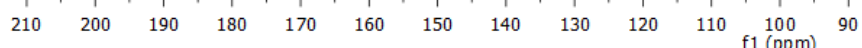
f1 (ppm) ${ }^{90} \quad 80$ 


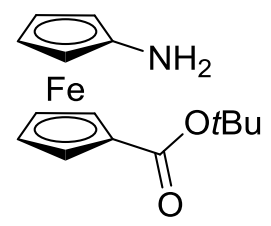

$5 d$

${ }^{1} \mathrm{H}$ NMR $\left(500 \mathrm{MHz}, \mathrm{CDCl}_{3}\right)$

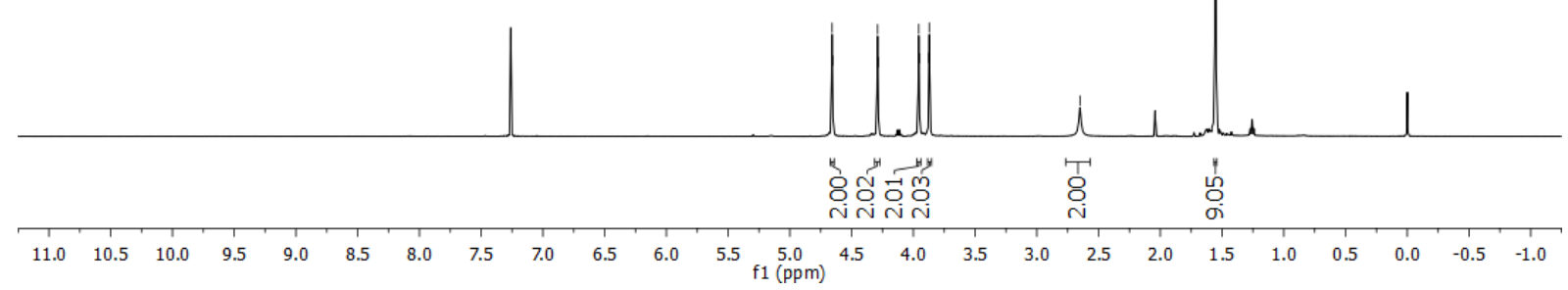

$\begin{array}{llll}\hat{8} & 0 & 0 & 0\end{array}$

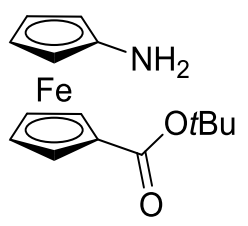

$5 d$

${ }^{13} \mathrm{C}\left\{{ }^{1} \mathrm{H}\right\} \mathrm{NMR}\left(126 \mathrm{MHz}, \mathrm{CDCl}_{3}\right)$

$210 \quad 200 \quad 190$

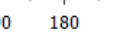

$140 \quad 130$

$30 \quad 120$

$10 \quad 100 \quad 90$ 


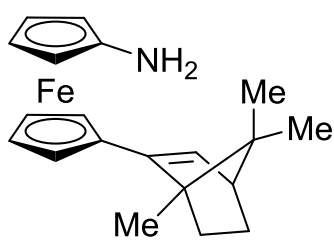

$5 e$

${ }^{1} \mathrm{H}$ NMR $\left(600 \mathrm{MHz}, \mathrm{CDCl}_{3}\right)$

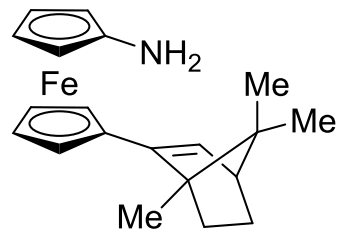

$5 e$

${ }^{13} \mathrm{C}\left\{{ }^{1} \mathrm{H}\right\} \operatorname{NMR}\left(151 \mathrm{MHz}, \mathrm{CDCl}_{3}\right)$

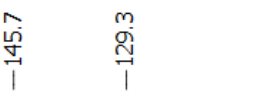

涡

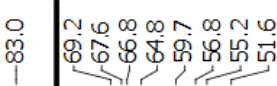

Nimo

ing

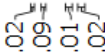

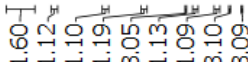

$\begin{array}{lllllllllllllllllllllllll}11.0 & 10.5 & 10.0 & 9.5 & 9.0 & 8.5 & 8.0 & 7.5 & 7.0 & 6.5 & 6.0 & 5.5 & 5.0 & 4.5 & 4.0 & 3.5 & 3.0 & 2.5 & 2.0 & 1.5 & 1.0 & 0.5 & 0.0 & -0.5 & -1.0\end{array}$

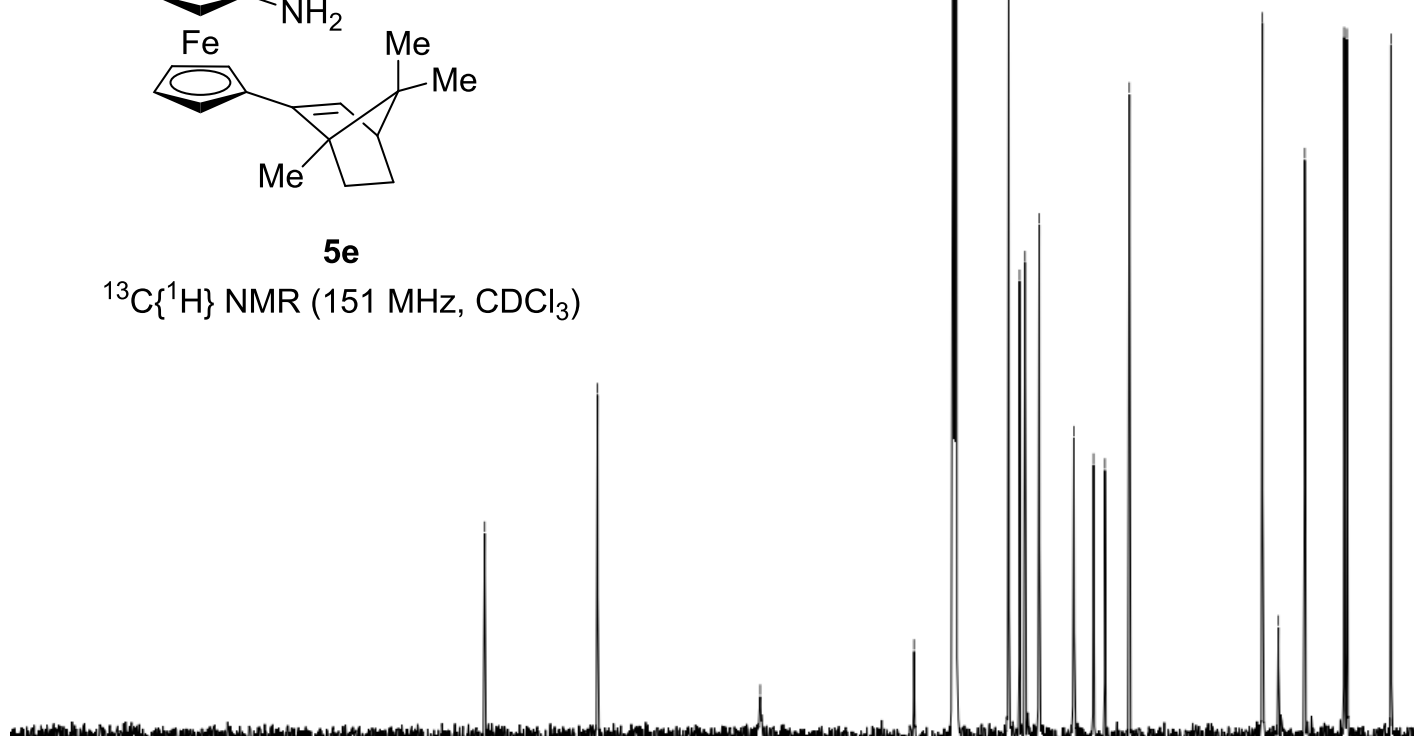

$\begin{array}{lllllllllll}200 & 190 & 180 & 170 & 160 & 150 & 140 & 130 & 120 & 110 & \begin{array}{c}100 \\ \mathrm{f} 1(\mathrm{ppm})\end{array}\end{array}$

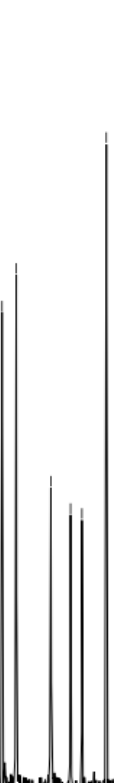




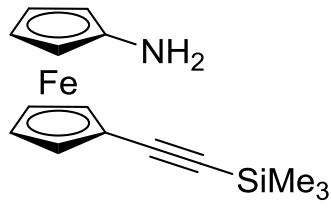

$5 f$

${ }^{1} \mathrm{H} \mathrm{NMR}\left(400 \mathrm{MHz}, \mathrm{CDCl}_{3}\right)$
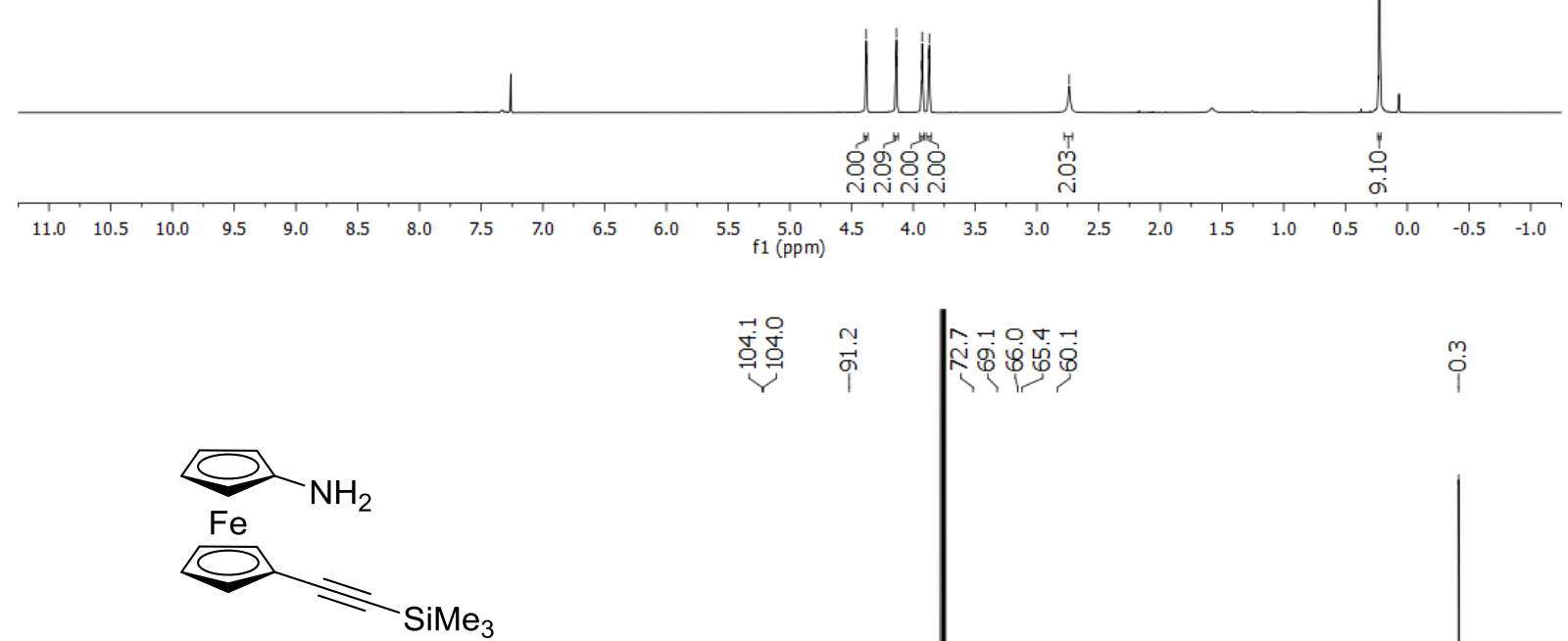

$5 f$

${ }^{13} \mathrm{C}\left\{{ }^{1} \mathrm{H}\right\} \operatorname{NMR}\left(100 \mathrm{MHz}, \mathrm{CDCl}_{3}\right)$

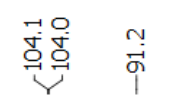

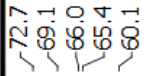

$\stackrel{m}{i}$

$\begin{array}{llllllllllll}210 & 200 & 190 & 180 & 170 & 160 & 150 & 140 & 130 & 120 & 110 & \underset{f 1}{100}(\mathrm{ppm})\end{array}$ 


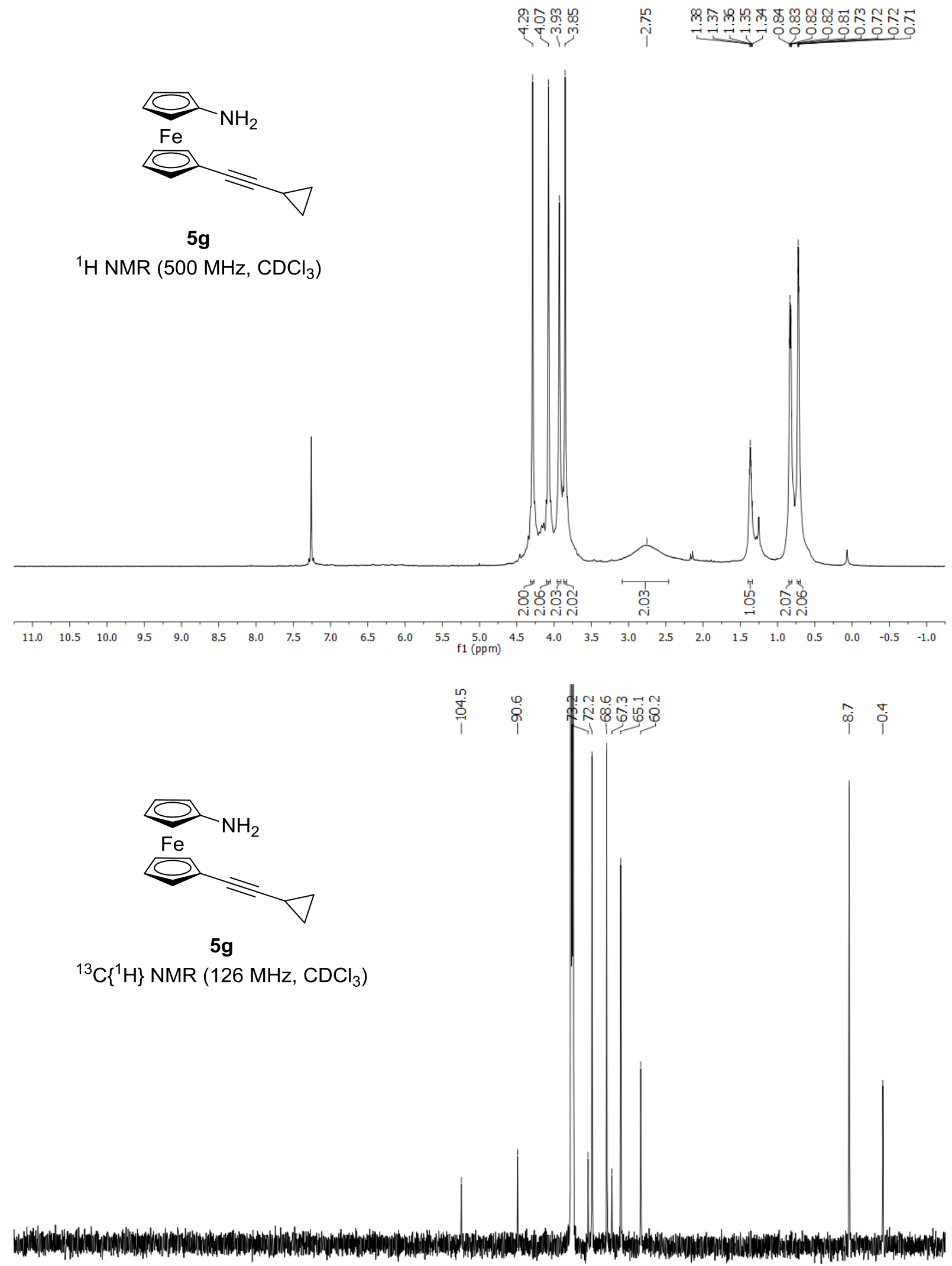

$\begin{array}{llllllllllll}210 & 200 & 190 & 180 & 170 & 160 & 150 & 140 & 130 & 120 & 110 & 100 \\ \mathrm{f} 1(\mathrm{ppm}) & 90\end{array}$ 


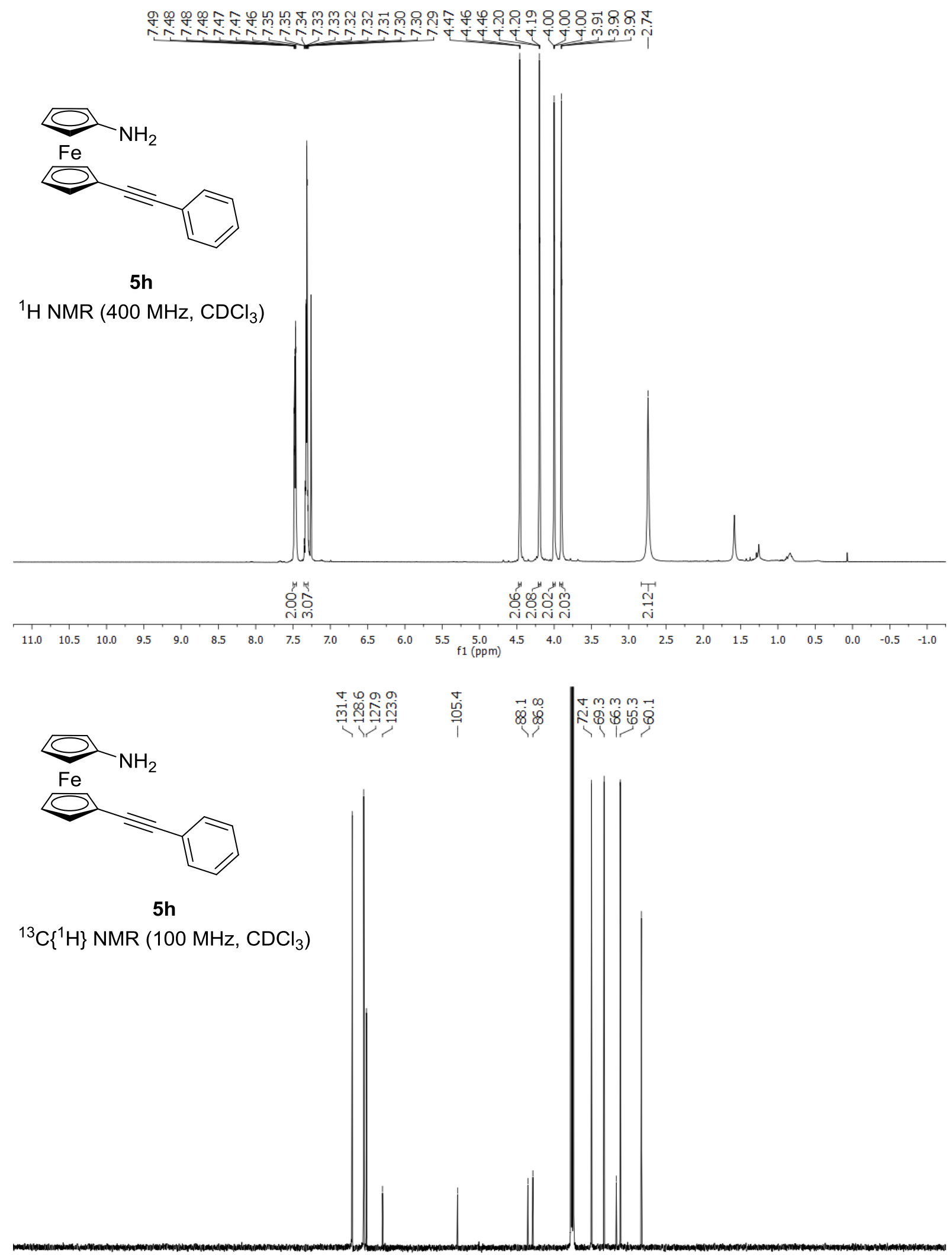

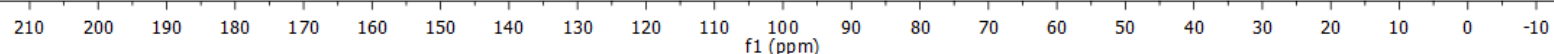




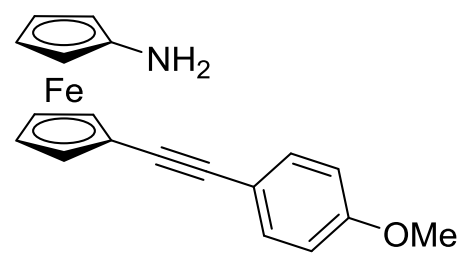

$5 i$

${ }^{1} \mathrm{H}$ NMR $\left(600 \mathrm{MHz}, \mathrm{CDCl}_{3}\right)$

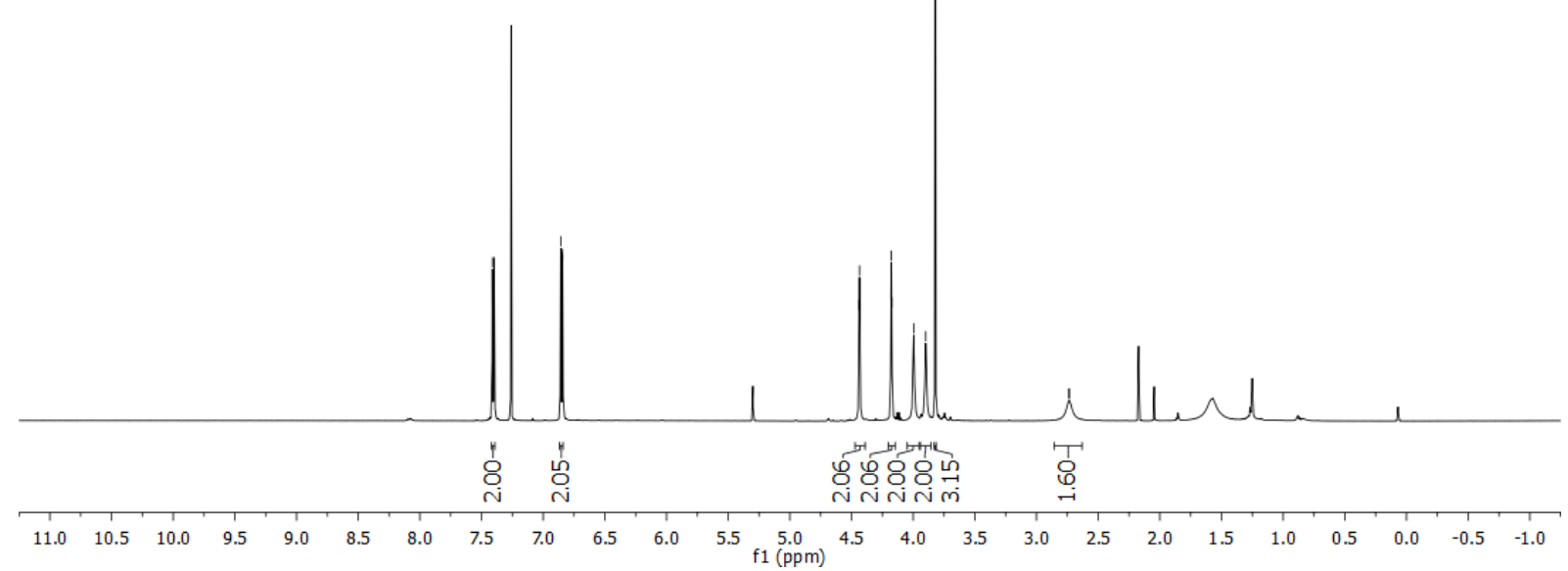

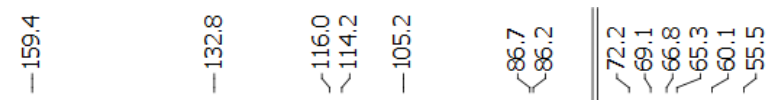

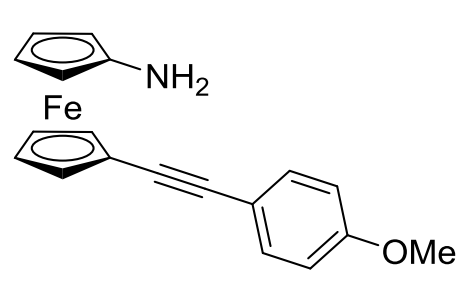

$5 i$

${ }^{13} \mathrm{C}\left\{{ }^{1} \mathrm{H}\right\} \operatorname{NMR}\left(151 \mathrm{MHz}, \mathrm{CDCl}_{3}\right)$

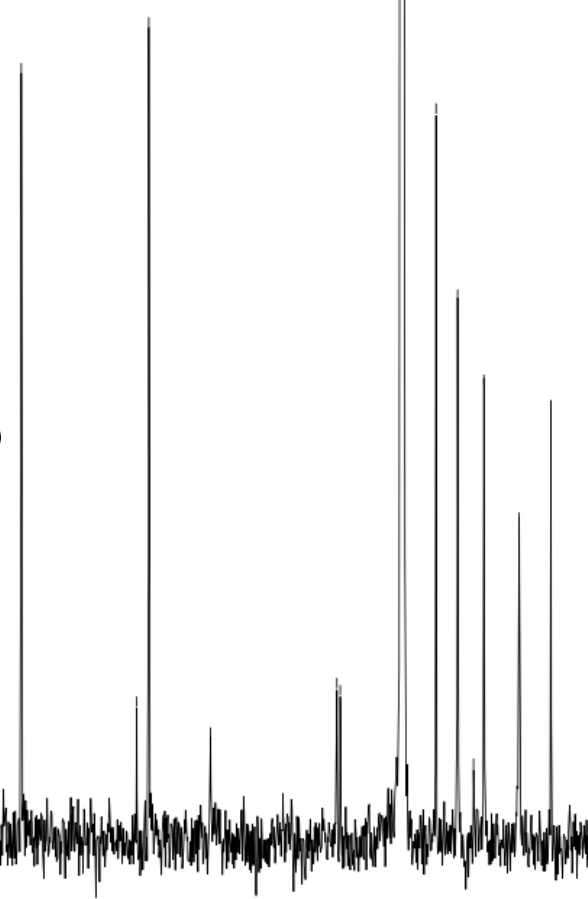

$210 \quad 200$

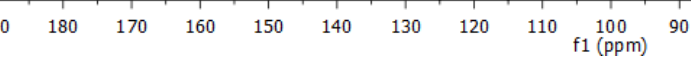

M.

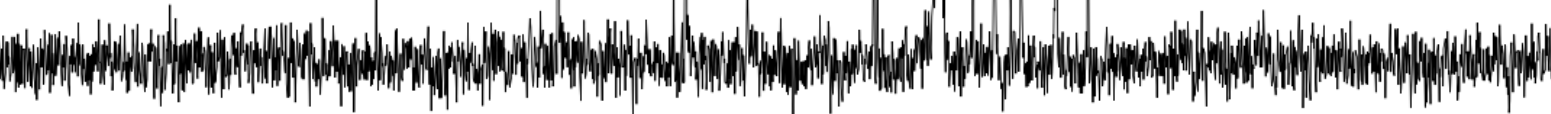

$\begin{array}{llllllllll}190 & 180 & 170 & 160 & 150 & 140 & 130 & 120 & 110 & \begin{array}{l}100 \\ \mathrm{f} 1(\mathrm{ppm})\end{array}\end{array}$




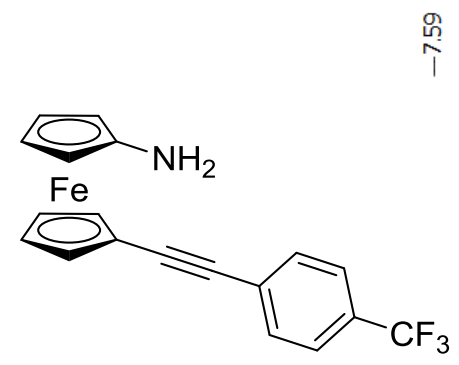

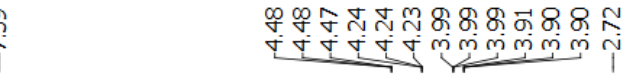

$5 j$

${ }^{1} \mathrm{H}$ NMR $\left(500 \mathrm{MHz}, \mathrm{CD}_{2} \mathrm{Cl}_{2}\right)$

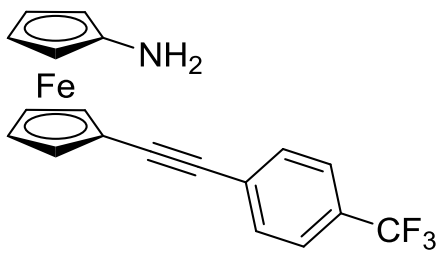

$5 \mathbf{j}$

${ }^{13} \mathrm{C}\left\{{ }^{1} \mathrm{H}\right\}$ NMR $\left(126 \mathrm{MHz}, \mathrm{CD}_{2} \mathrm{Cl}_{2}\right)$

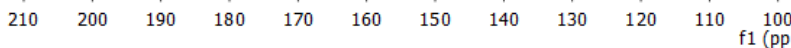
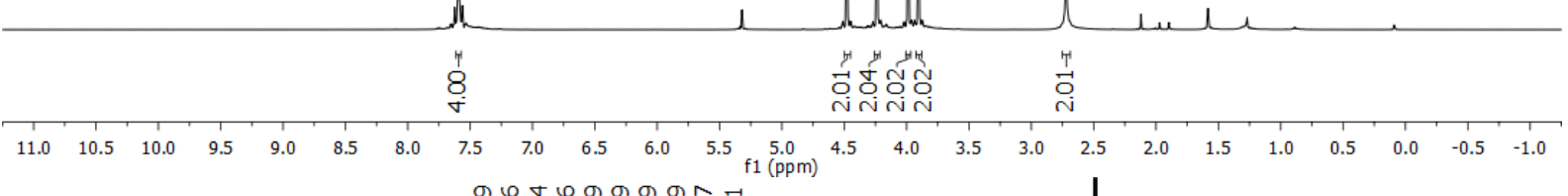

m

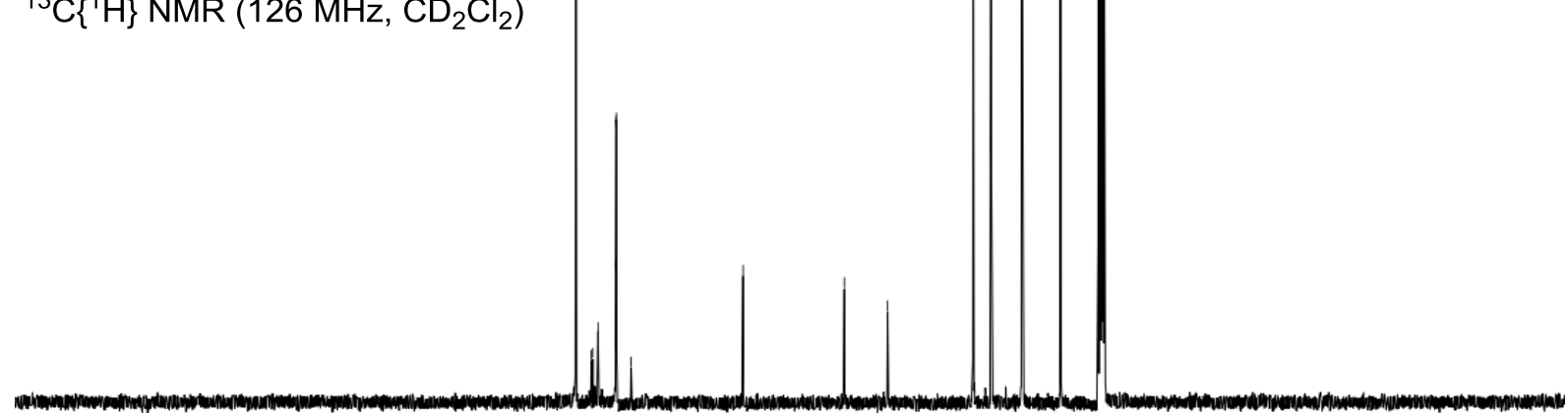




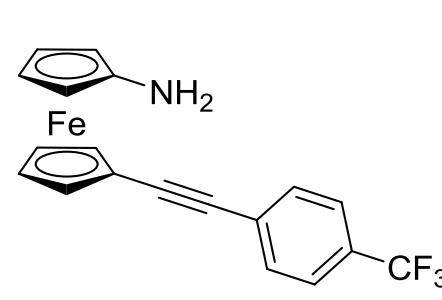

$5 j$

${ }^{19} \mathrm{~F}$ NMR $\left(471 \mathrm{MHz}, \mathrm{CD}_{2} \mathrm{Cl}_{2}\right)$

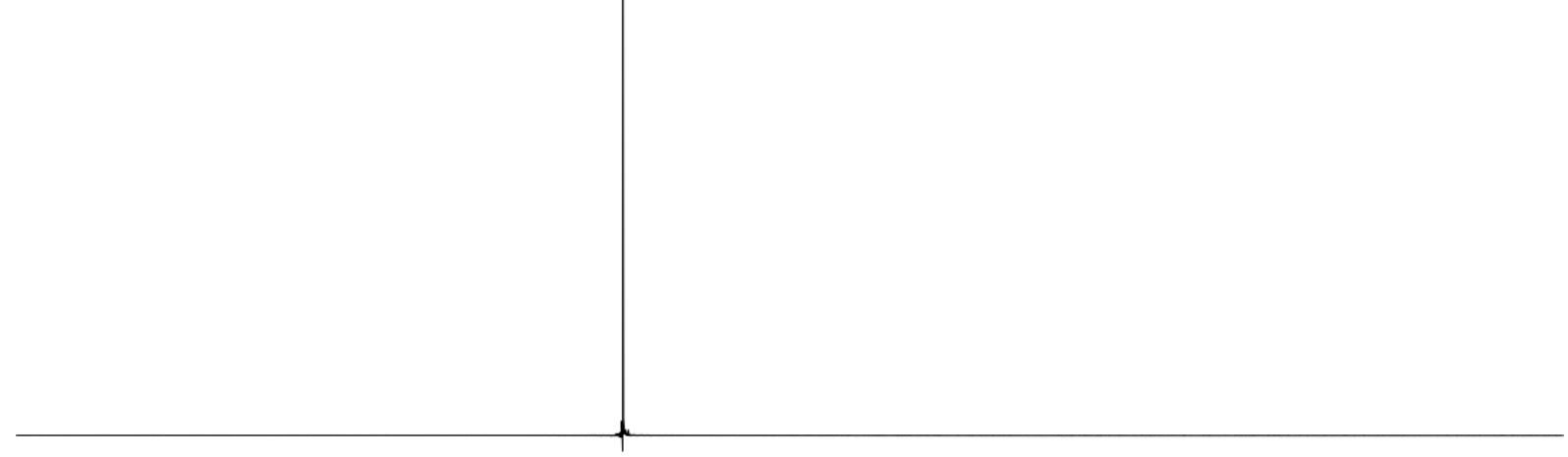

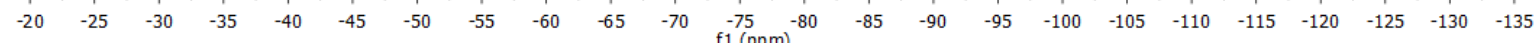




\subsection{NMR Spectra of Compounds Synthesized in Flow}

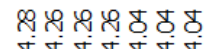

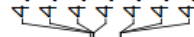

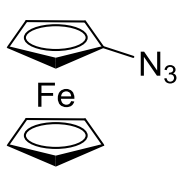

$2 a$

${ }^{1} \mathrm{H}$ NMR $\left(700 \mathrm{MHz}, \mathrm{CDCl}_{3}\right)$

880
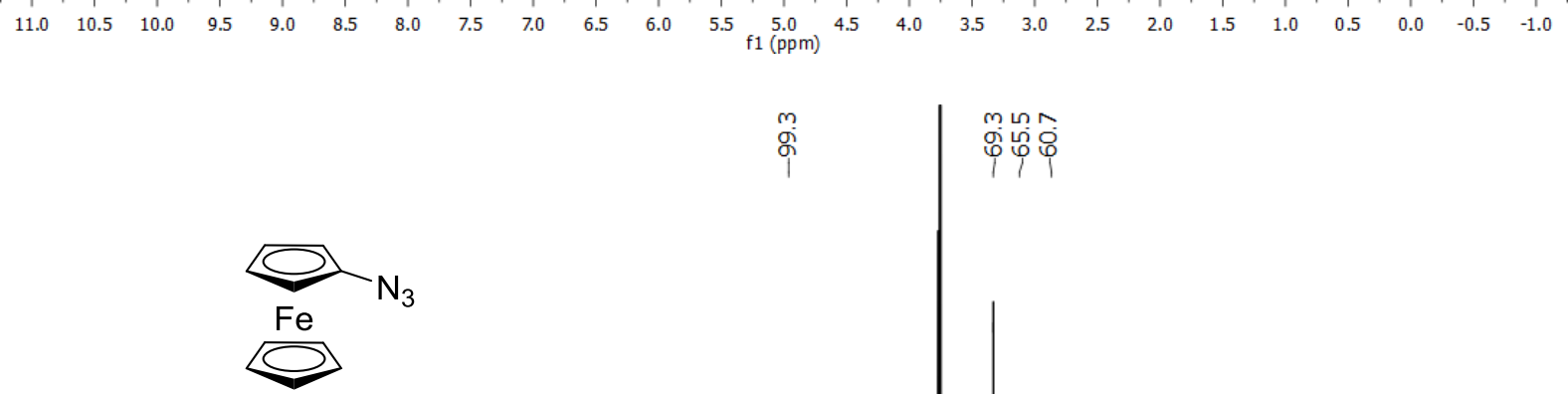

$2 a$

${ }^{13} \mathrm{C}\left\{{ }^{1} \mathrm{H}\right\}$ NMR $\left(176 \mathrm{MHz}, \mathrm{CDCl}_{3}\right)$

$\begin{array}{llllllllllll}210 & 200 & 190 & 180 & 170 & 160 & 150 & 140 & 130 & 120 & 110 & \begin{array}{l}100 \\ \mathrm{f} 1(\mathrm{ppm})\end{array}\end{array}$ 


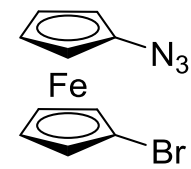

2b

${ }^{1} \mathrm{H}$ NMR $\left(700 \mathrm{MHz}, \mathrm{CDCl}_{3}\right)$
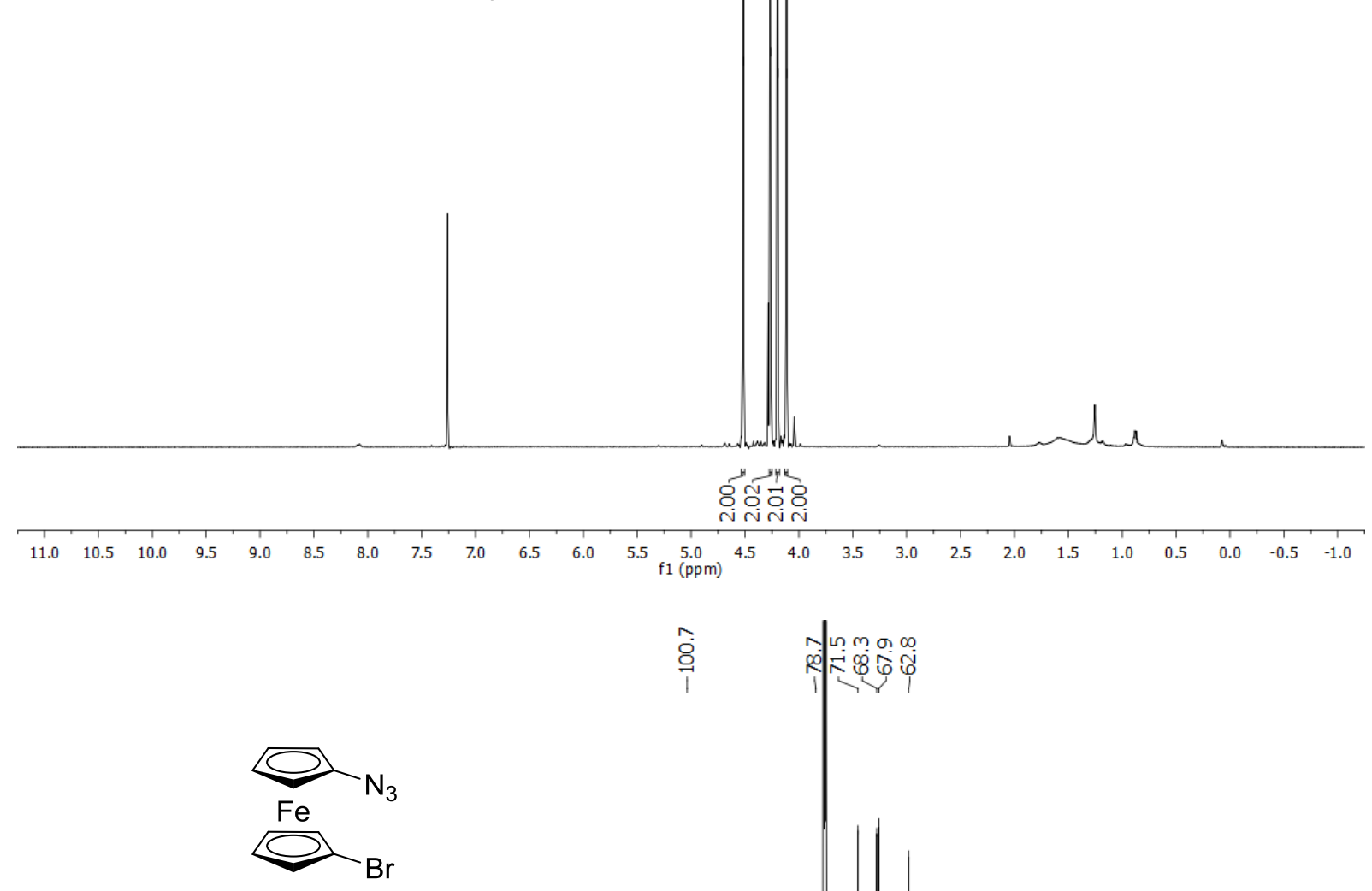

2b

${ }^{13} \mathrm{C}\left\{{ }^{1} \mathrm{H}\right\}$ NMR $\left(176 \mathrm{MHz}, \mathrm{CDCl}_{3}\right)$
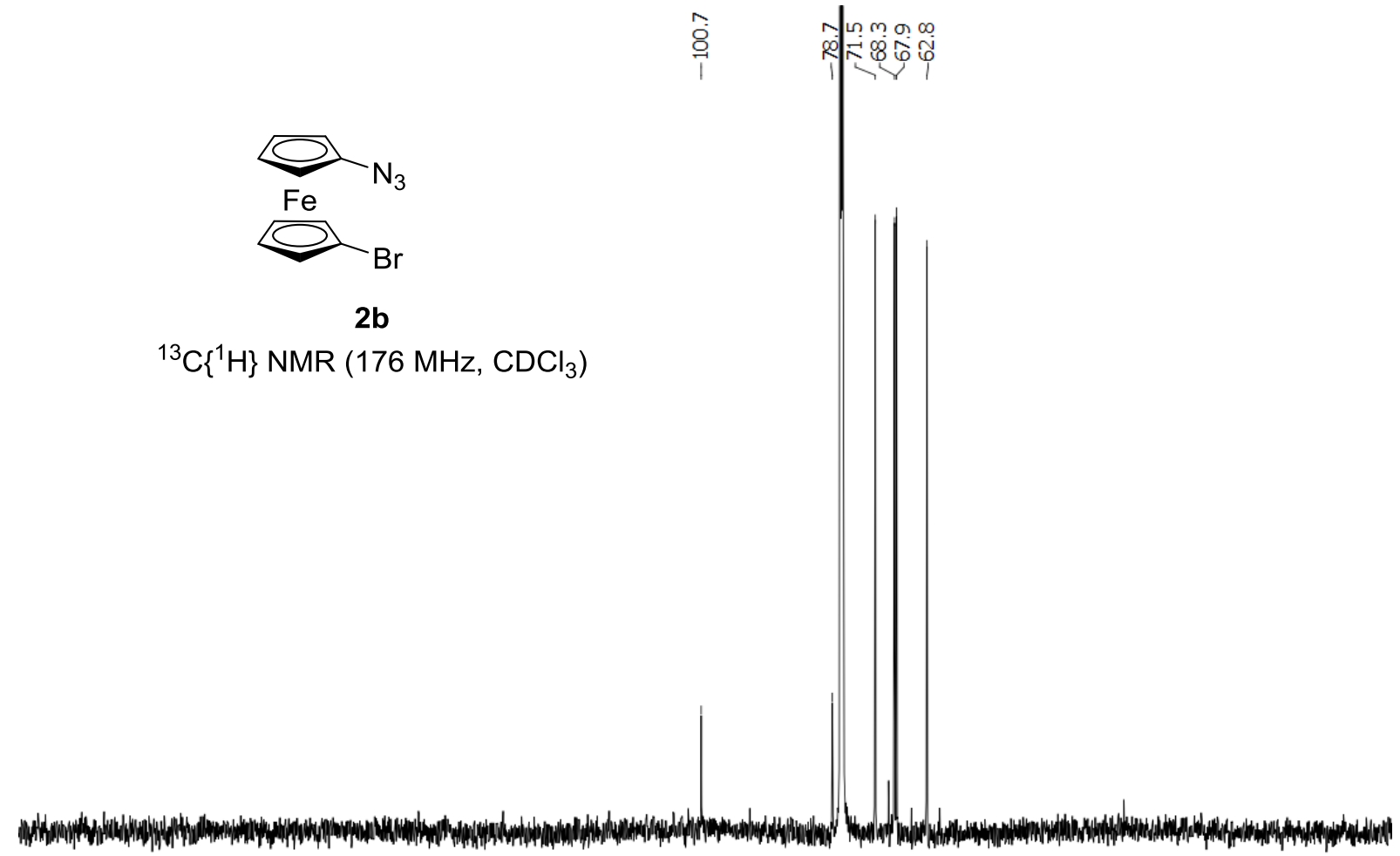

$\begin{array}{llllllllllllllllllllllllll}210 & 200 & 190 & 180 & 170 & 160 & 150 & 140 & 130 & 120 & 110 & 100 & 90 & 80 & 70 & 60 & 50 & 40 & 30 & 20 & 10 & 0 & -10\end{array}$ 


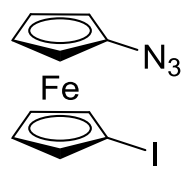

2c

${ }^{1} \mathrm{H}$ NMR $\left(600 \mathrm{MHz}, \mathrm{CDCl}_{3}\right)$

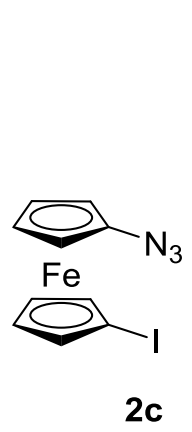

2c

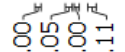

$\begin{array}{llllllllllllllllllllllllll}11.0 & 10.5 & 10.0 & 9.5 & 9.0 & 8.5 & 8.0 & 7.5 & 7.0 & 6.5 & 6.0 & 5.5 & \begin{array}{c}5 \\ \mathrm{f} 1.0\end{array} & 4.5 & 4.0 & 3.5 & 3.0 & 2.5 & 2.0 & 1.5 & 1.0 & 0.5 & 0.0 & -0.5 & -1.0\end{array}$

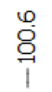

당요 응

ํ.

$\stackrel{9}{\circ}$

${ }^{13} \mathrm{C}\left\{{ }^{1} \mathrm{H}\right\}$ NMR (151 MHz, $\left.\mathrm{CDCl}_{3}\right)$ 


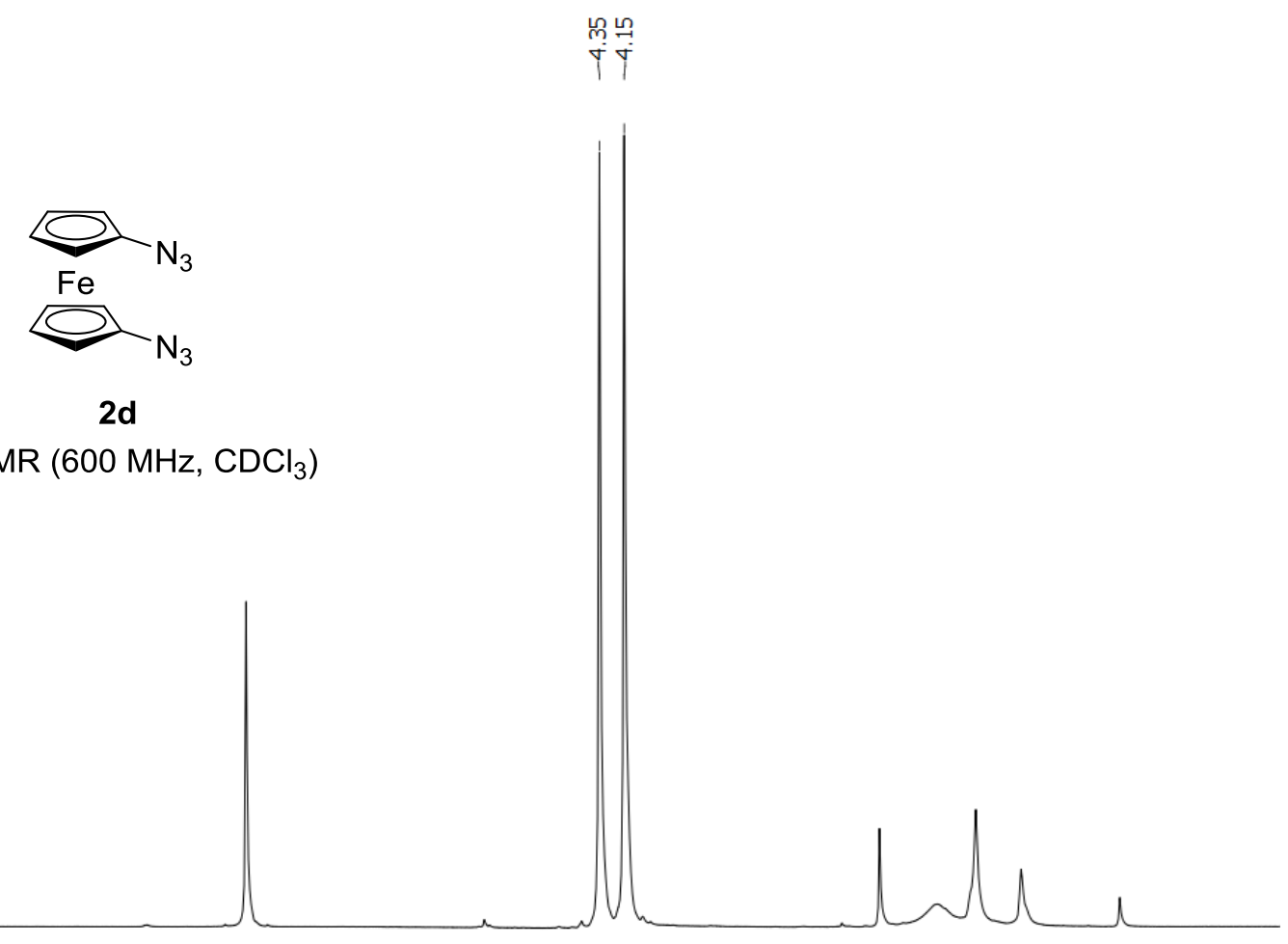

策管
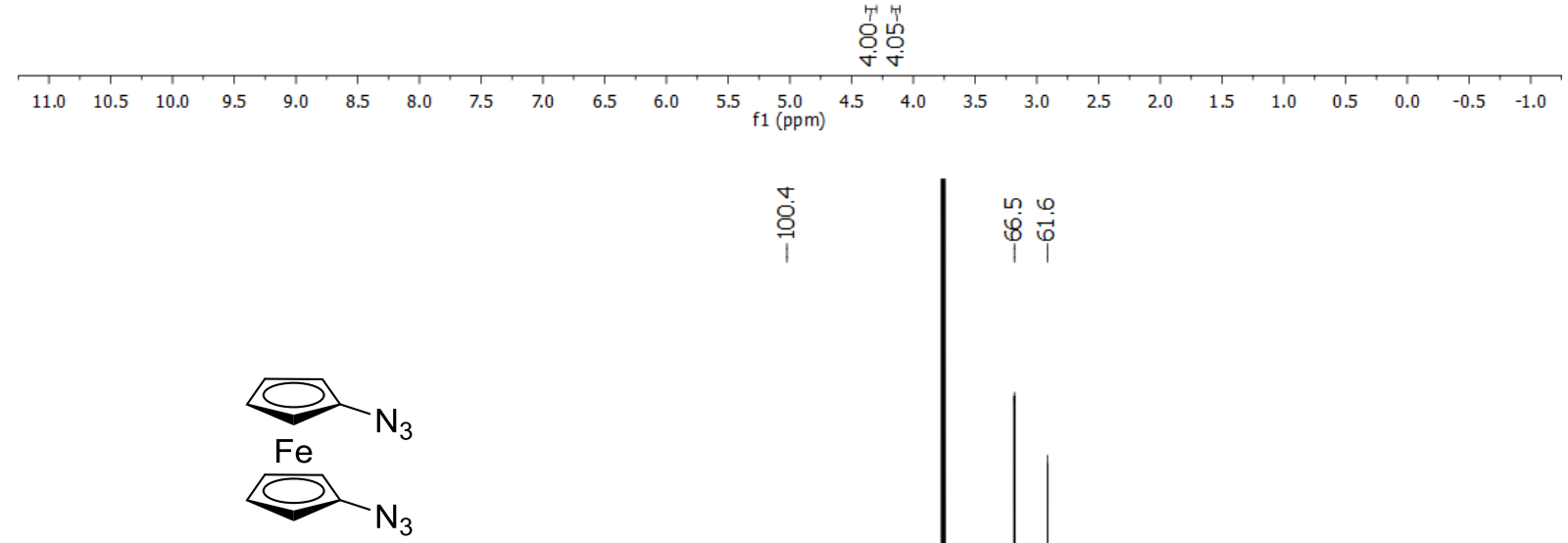

2d

${ }^{13} \mathrm{C}\left\{{ }^{1} \mathrm{H}\right\} \operatorname{NMR}\left(151 \mathrm{MHz}, \mathrm{CDCl}_{3}\right)$

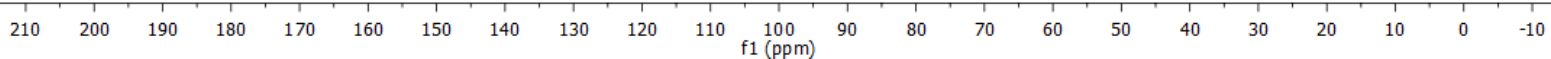




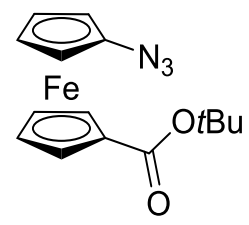

$2 e$

${ }^{1} \mathrm{H}$ NMR $\left(700 \mathrm{MHz}, \mathrm{CDCl}_{3}\right)$

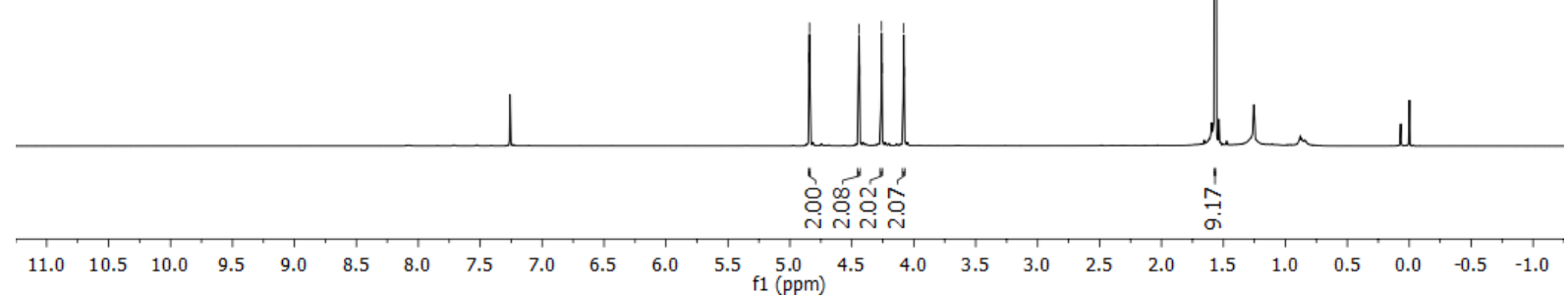

$\stackrel{m}{\stackrel{m}{1}}$

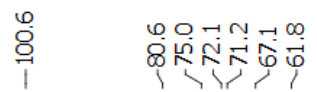

$\stackrel{1}{\infty}$

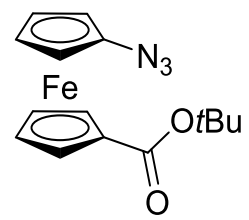

$2 \mathrm{e}$

${ }^{13} \mathrm{C}\left\{{ }^{1} \mathrm{H}\right\}$ NMR (176 MHz, $\mathrm{CDCl}_{3}$ )

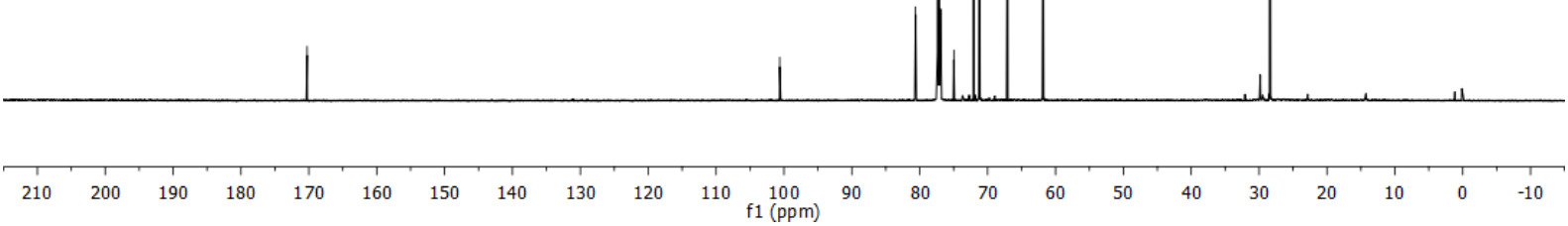




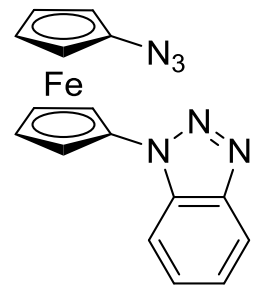

$2 f$

${ }^{1} \mathrm{H}$ NMR $\left(600 \mathrm{MHz}, \mathrm{CDCl}_{3}\right)$

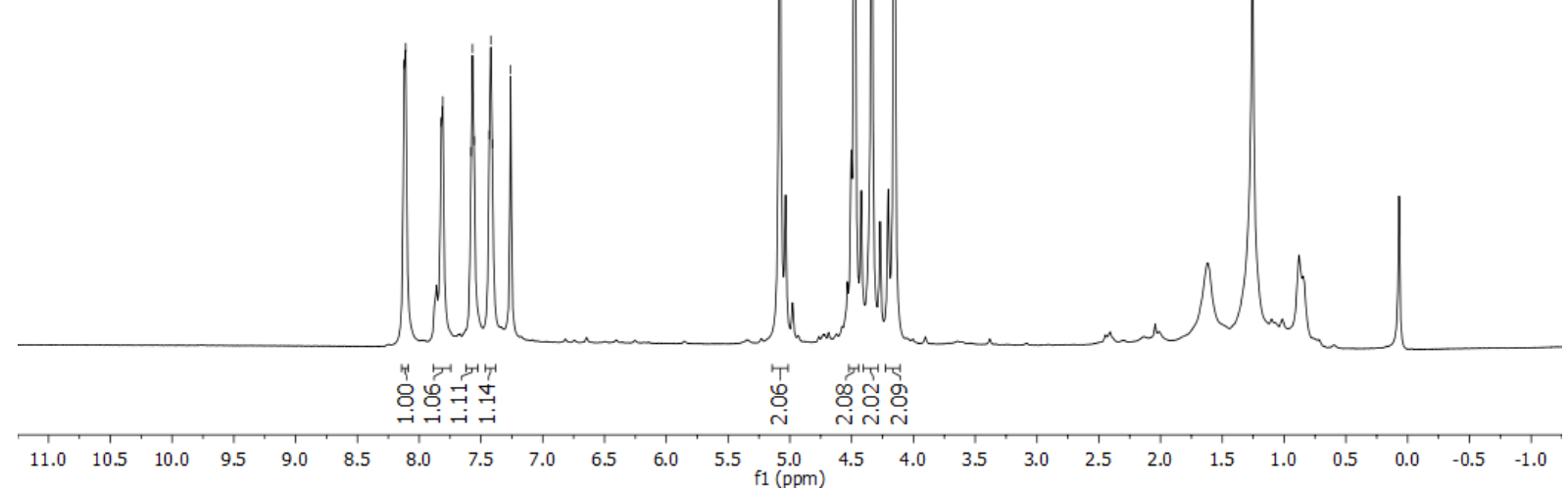

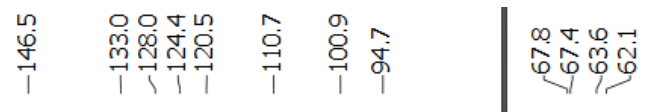

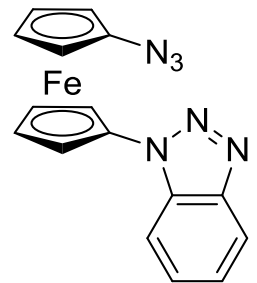

$2 f$

${ }^{13} \mathrm{C}\left\{{ }^{1} \mathrm{H}\right\}$ NMR $\left(151 \mathrm{MHz}, \mathrm{CDCl}_{3}\right)$

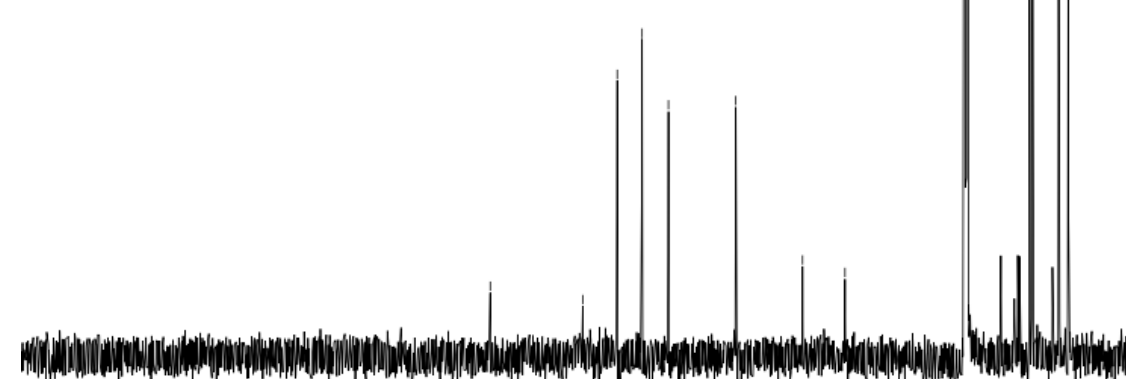

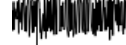

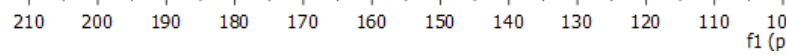




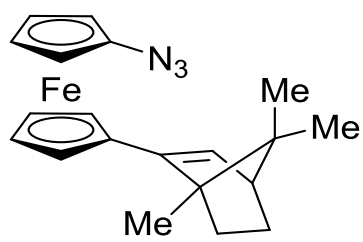

$2 \mathbf{g}$

${ }^{1} \mathrm{H}$ NMR $\left(600 \mathrm{MHz}, \mathrm{CDCl}_{3}\right)$
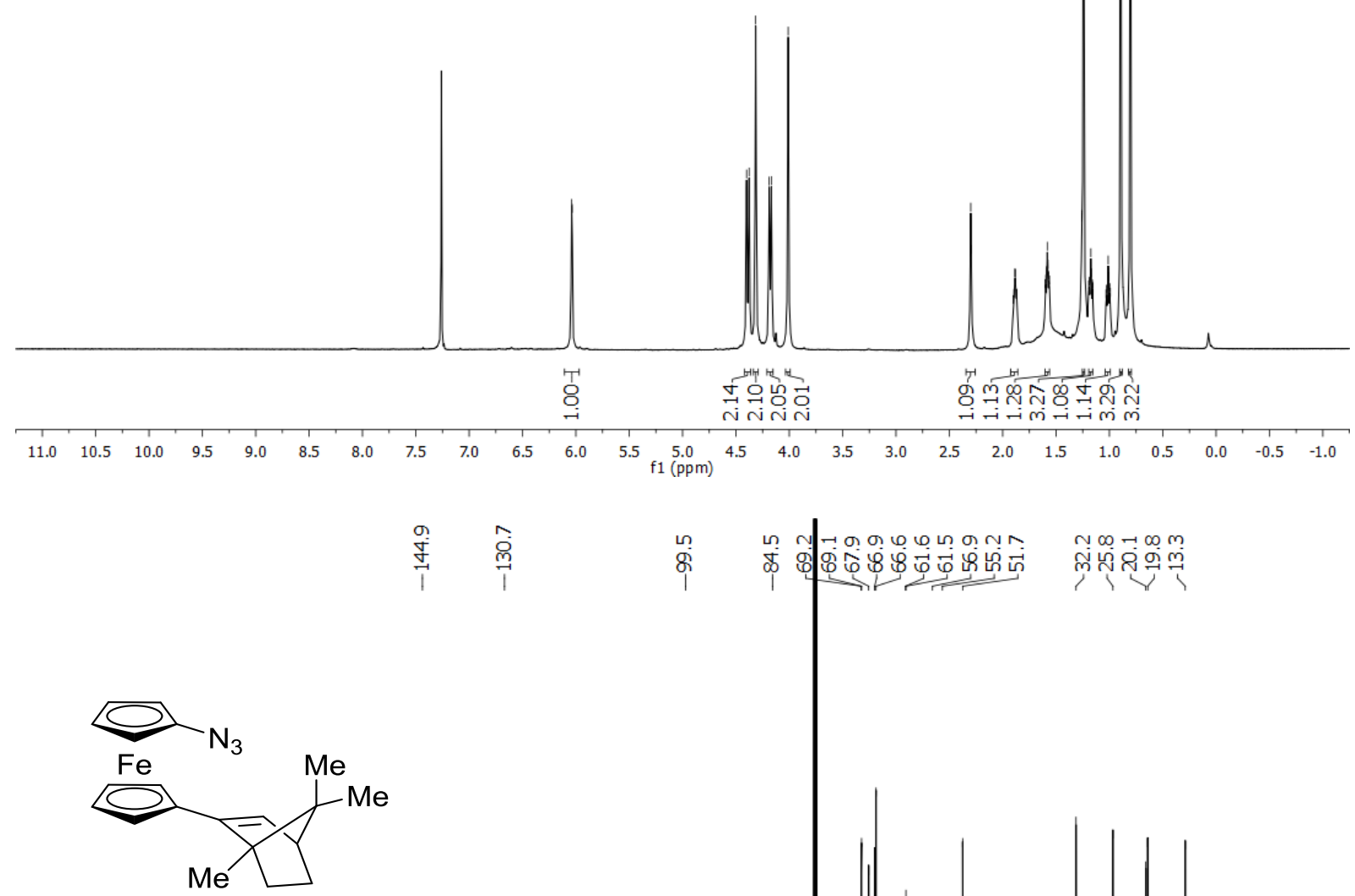

2g

${ }^{13} \mathrm{C}\left\{{ }^{1} \mathrm{H}\right\}$ NMR $\left(151 \mathrm{MHz}, \mathrm{CDCl}_{3}\right)$

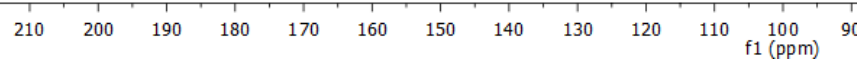




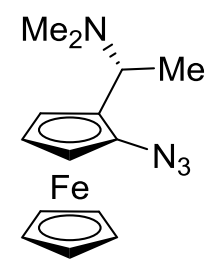

$2 \mathrm{~h}$

${ }^{1} \mathrm{H}$ NMR $\left(500 \mathrm{MHz}, \mathrm{CDCl}_{3}\right.$ )

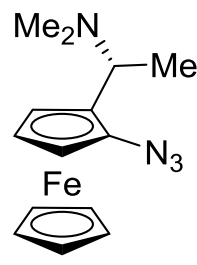

$2 h$

$\left.{ }^{13} \mathrm{C}\left\{{ }^{1} \mathrm{H}\right\} \mathrm{NMR}(126 \mathrm{MHz}, \mathrm{CDCl})_{3}\right)$

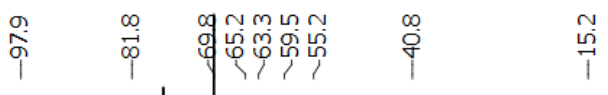

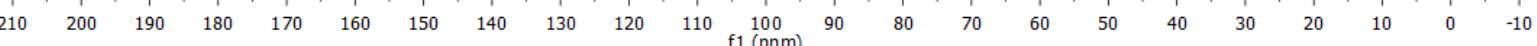




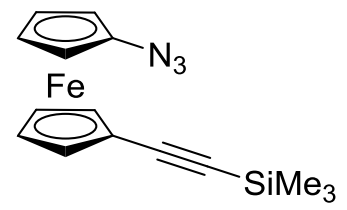

2i

${ }^{1} \mathrm{H}$ NMR (700 MHz, $\mathrm{CDCl}_{3}$ )
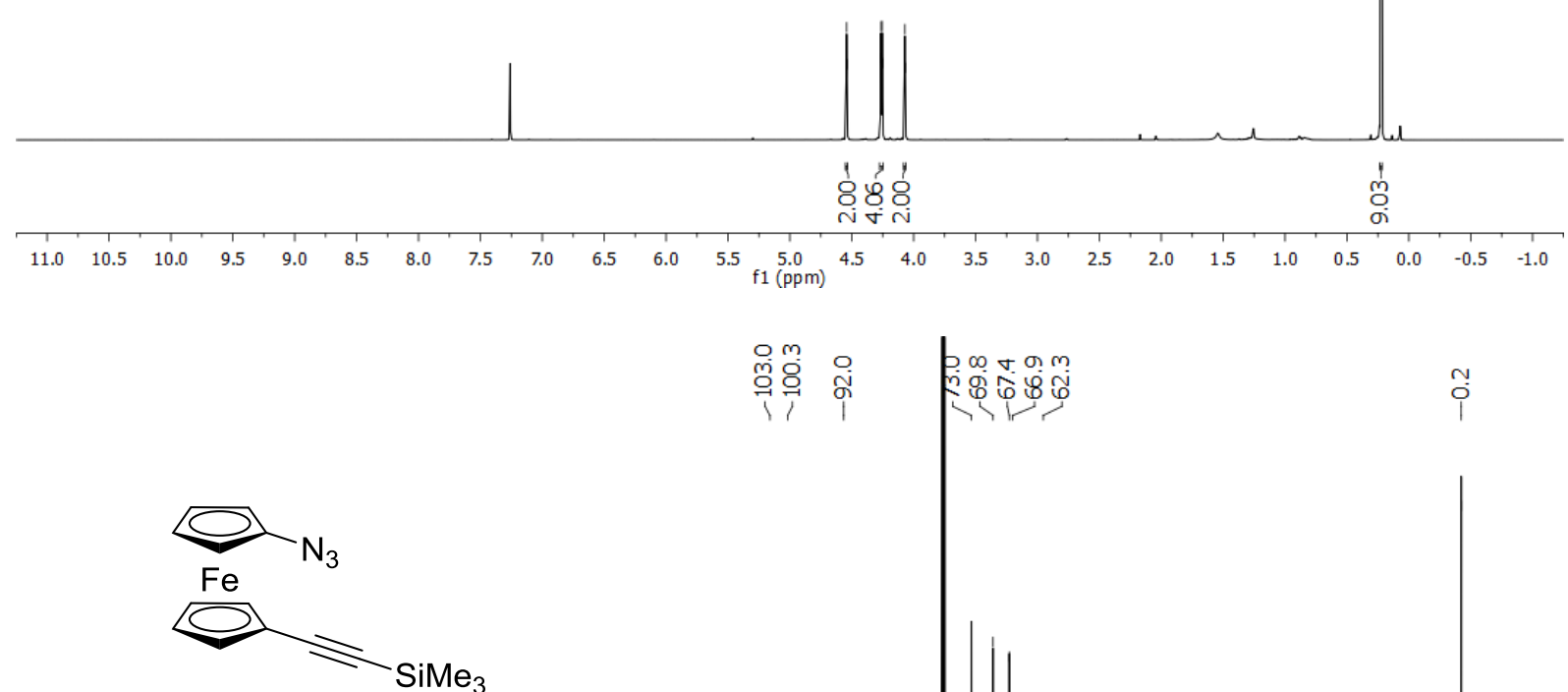

2i

${ }^{13} \mathrm{C}\left\{{ }^{1} \mathrm{H}\right\}$ NMR (176 MHz, $\left.\mathrm{CDCl}_{3}\right)$

$\begin{array}{ll}0 & m \\ \text { mo } & 0 \\ 0 & 0 \\ 1 & 1\end{array}$

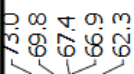

$\stackrel{1}{1}$

$210 \quad 200$

$\begin{array}{lllllll}100 & 190 & 180 & 170 & 160 & 150 & 140\end{array}$

$\begin{array}{lll}40 & 130 & 120\end{array}$

$110 \underset{f 1(\mathrm{ppm})}{100} 90$

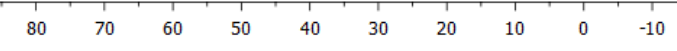




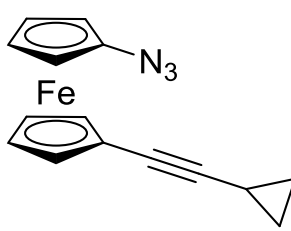

2j

${ }^{1} \mathrm{H}$ NMR $\left(600 \mathrm{MHz}, \mathrm{CDCl}_{3}\right)$

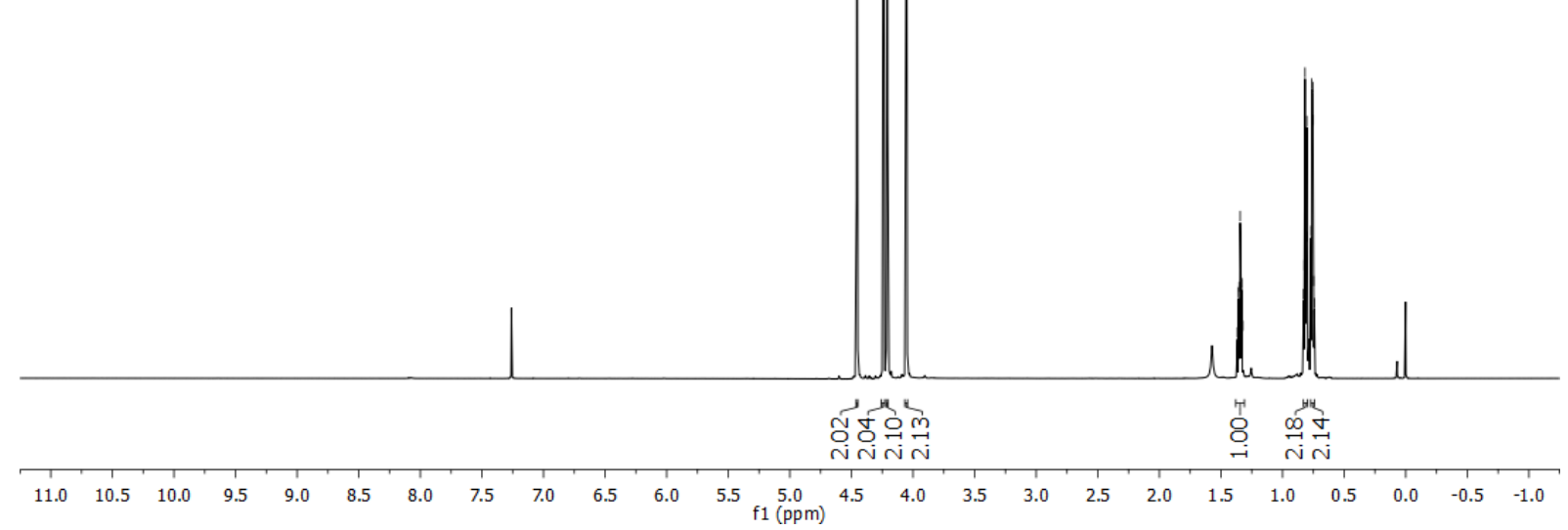

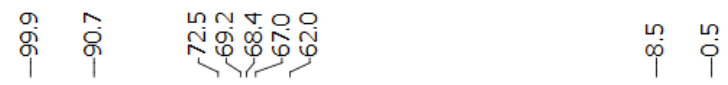

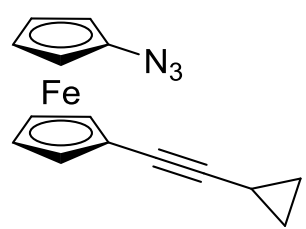

2j

${ }^{13} \mathrm{C}\left\{{ }^{1} \mathrm{H}\right\} \mathrm{NMR}\left(151 \mathrm{MHz}, \mathrm{CDCl}_{3}\right)$

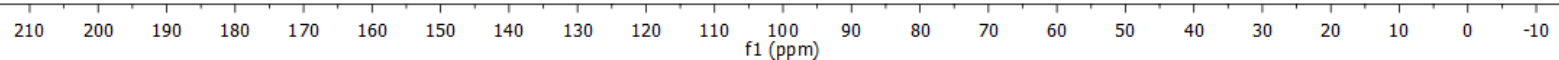



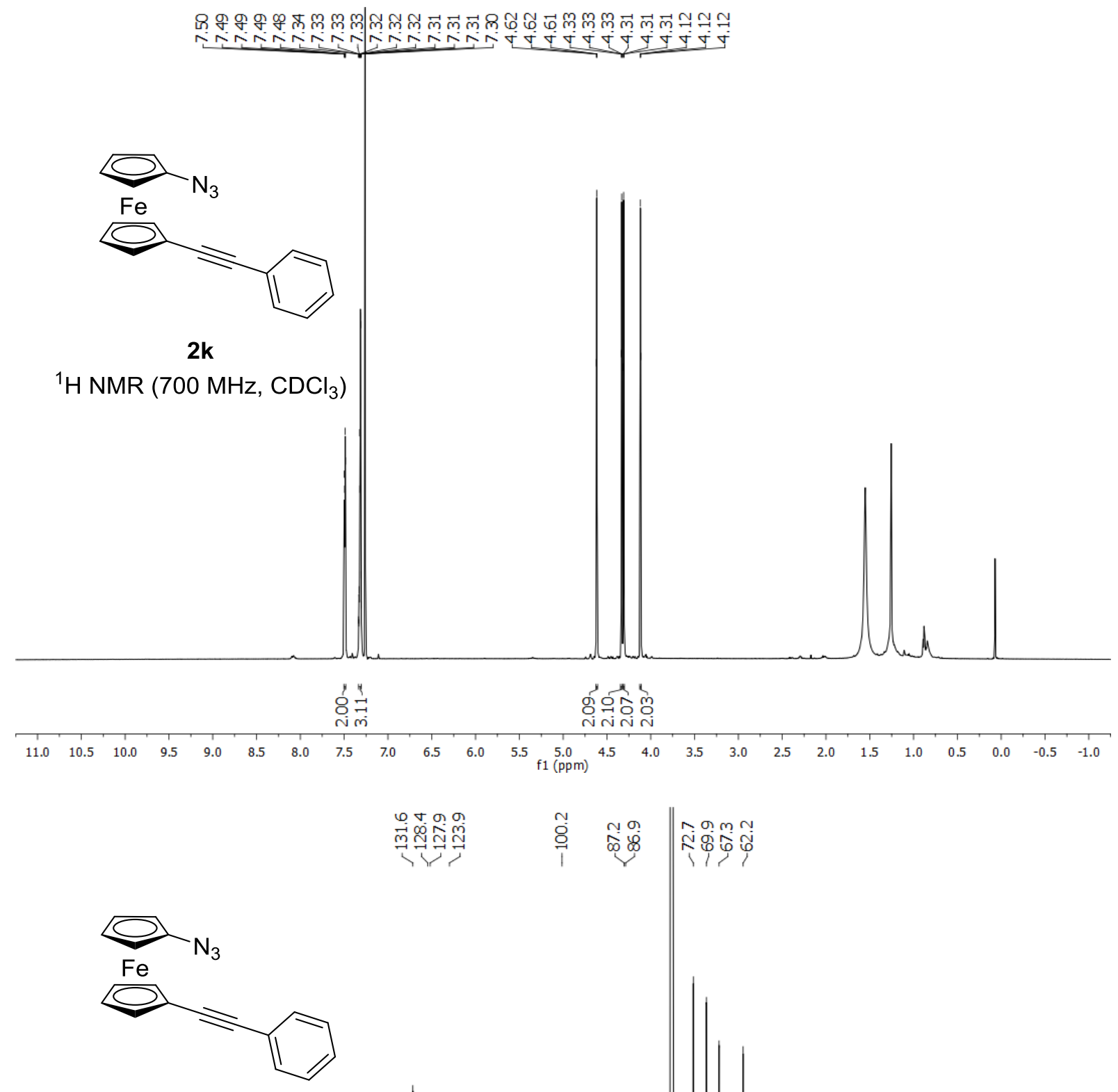

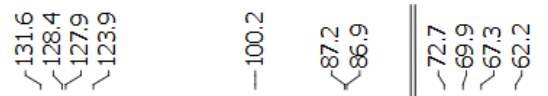

2k

$\left.{ }^{13} \mathrm{C}\left\{{ }^{1} \mathrm{H}\right\} \mathrm{NMR}(176 \mathrm{MHz}, \mathrm{CDCl})_{3}\right)$

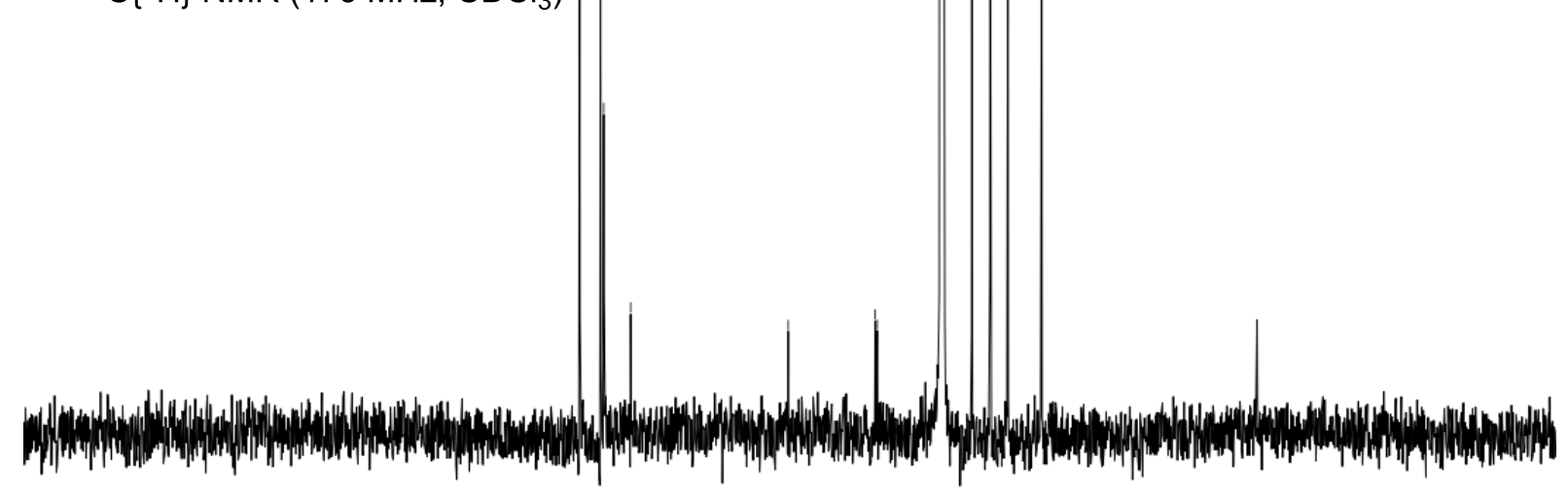

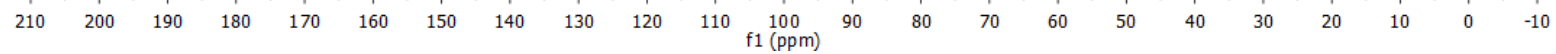




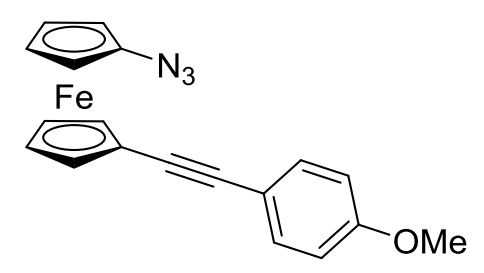

2I

${ }^{1} \mathrm{H}$ NMR $\left(700 \mathrm{MHz}, \mathrm{CDCl}_{3}\right)$

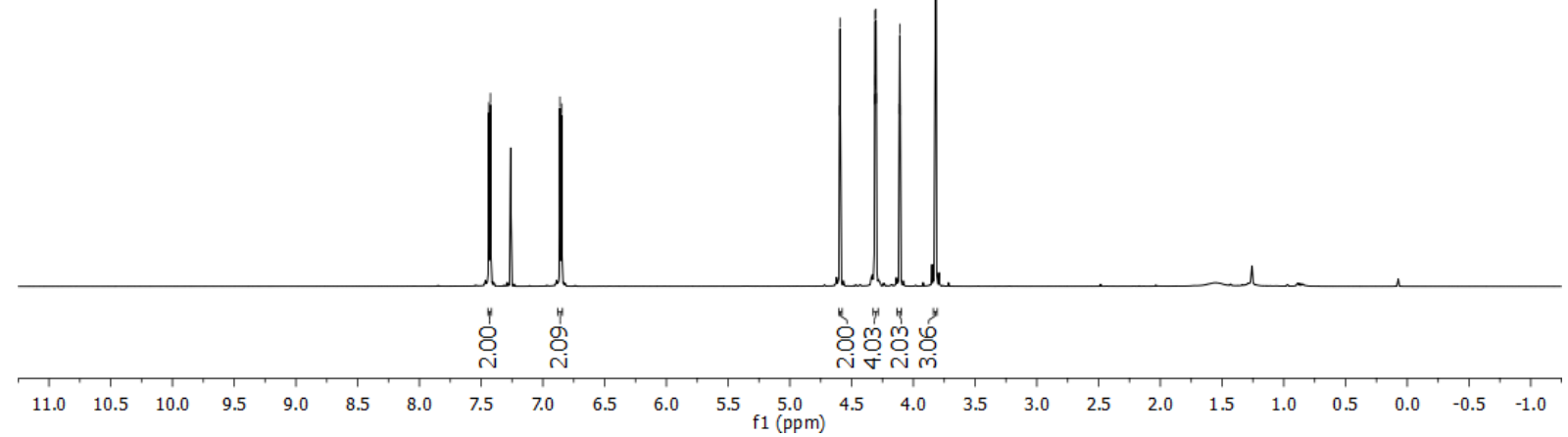

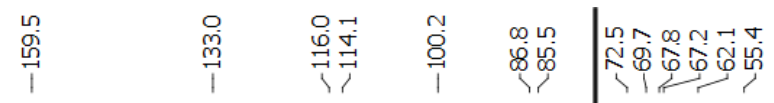

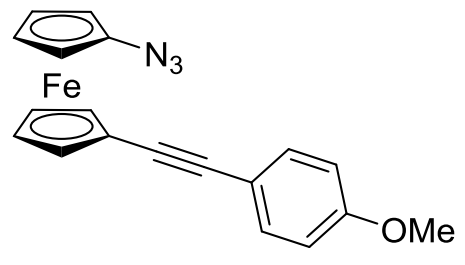

2I

${ }^{13} \mathrm{C}\left\{{ }^{1} \mathrm{H}\right\}$ NMR $\left(176 \mathrm{MHz}, \mathrm{CDCl}_{3}\right)$ 

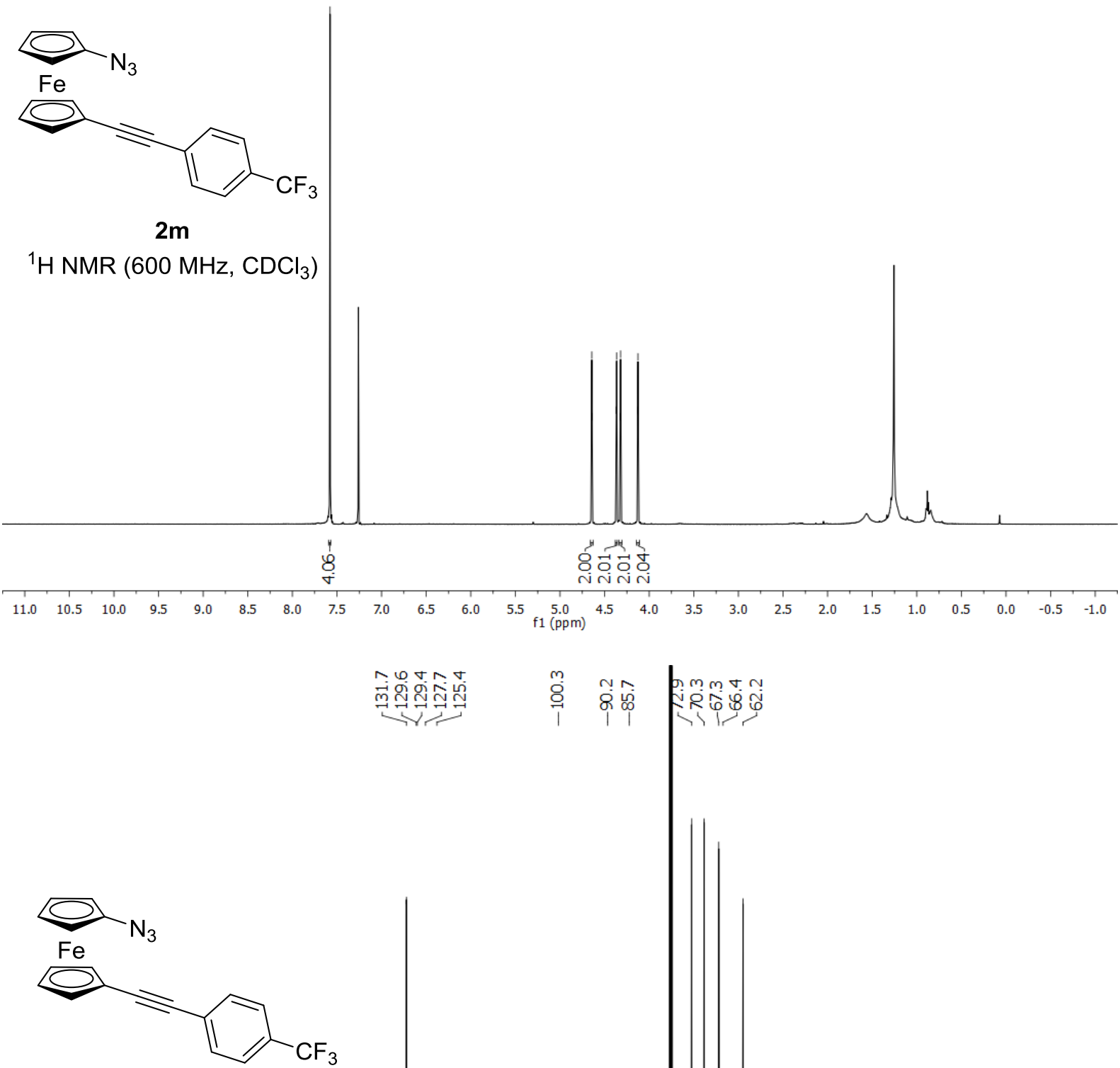

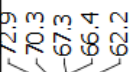

$2 \mathrm{~m}$

${ }^{13} \mathrm{C}\left\{{ }^{1} \mathrm{H}\right\}$ NMR $\left(151 \mathrm{MHz}, \mathrm{CDCl}_{3}\right)$

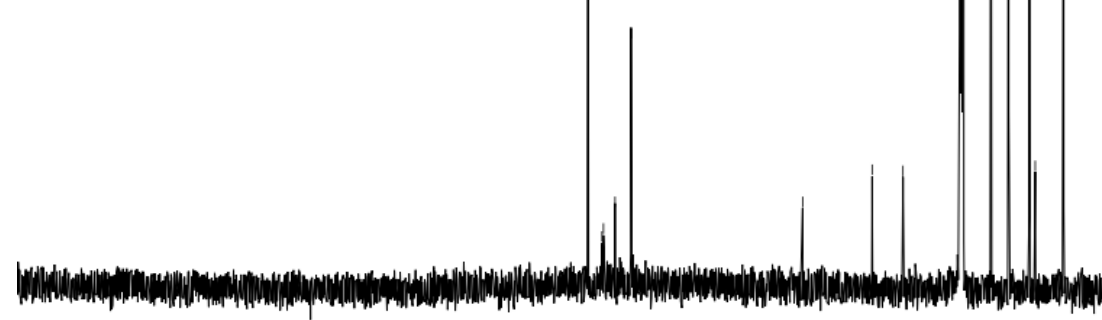

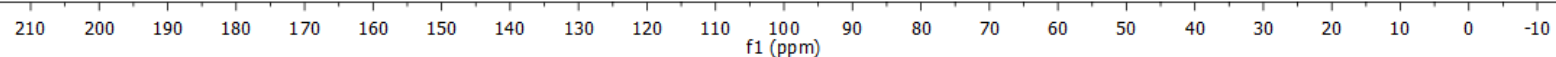




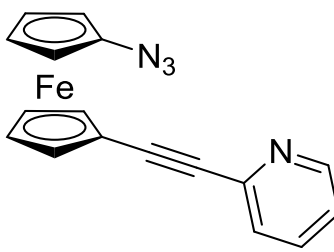

2n

${ }^{1} \mathrm{H}$ NMR (600 MHz, $\left.\mathrm{CDCl}_{3}\right)$

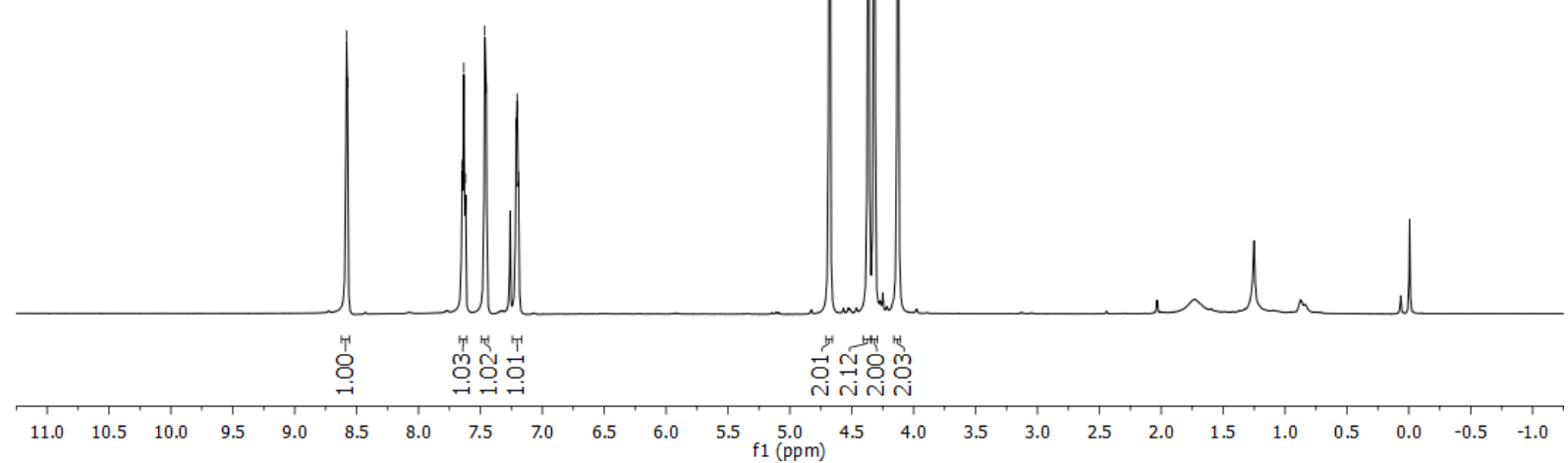

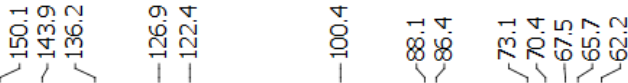

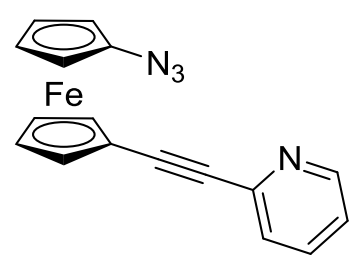

2n

${ }^{13} \mathrm{C}\left\{{ }^{1} \mathrm{H}\right\}$ NMR $\left(151 \mathrm{MHz}, \mathrm{CDCl}_{3}\right)$

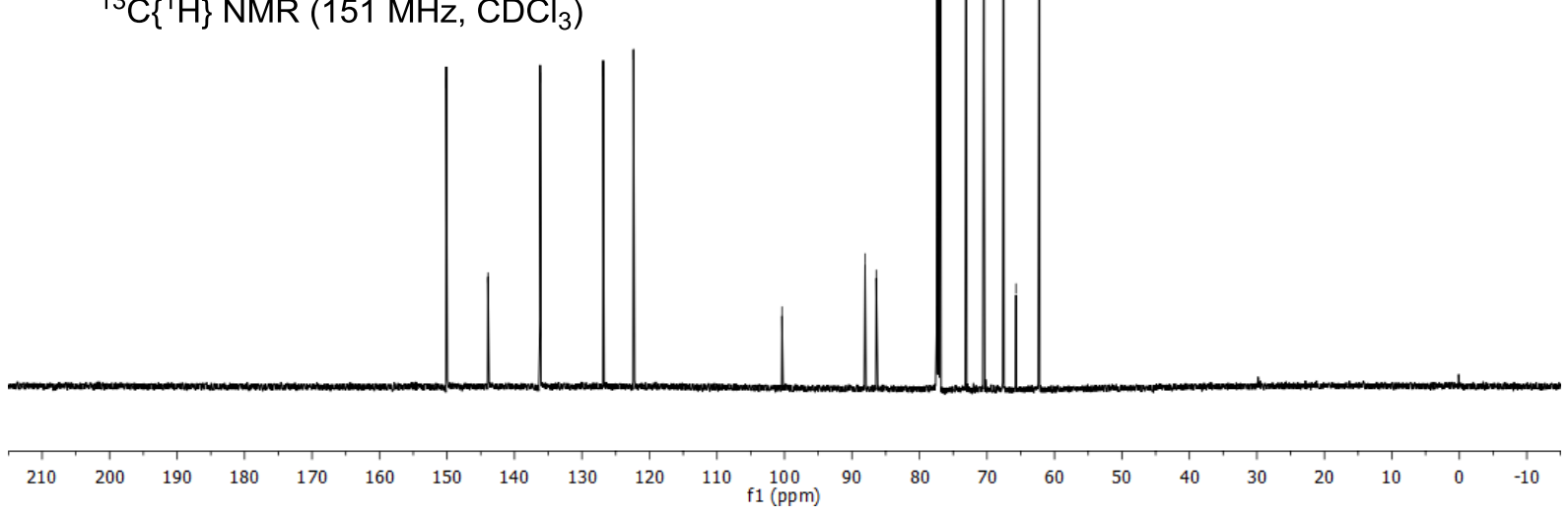




\section{References}

[1] S. Diethelm, C. S. Schindler, E. M. Carreira, Chem. Eur. J. 2014, 20, 6071-6080.

[2] N. N. Bhuvan Kumar, O. A. Mukhina, A. G. Kutateladze, J. Am. Chem. Soc. 2013, 135, 9608-9611.

[3] The original setup was developed by M. Spano from the Croatt research group, is open source and can be found on: https://chem.uncg.edu/croatt/flow-chemistry/ (25.02.19). For a recent application of this setup, see: L. Fakhouri, C. D. Cook, M. H. Al-Huniti, L. M. Console-Bram, D. P. Hurst, M. B. S. Spano, D. J. Nasrallah, M. G. Caron, L. S. Barak, P. H. Reggio, M. Abood, M. P. Croatt, Biorg. Med. Chem. 2017, 25, 4355-4367.

[4] B. Pieber, M. Shalom, M. Antonietti, P. H. Seeberger, K. Gilmore, Angew. Chem. Int. Ed. 2018, 57, 9976-9979; Angew. Chem. 2018, 130, 10127-10131.

[5] D. A. Khobragade, S. G. Mahamulkar, L. Pospíšil, I. Císařova, L. Rulíšek, U. Jahn, Chem. Eur. J. 2012, 18, 12267-12277.

[6] T. Okitsu, K. Sato, K. Iwatsuka, N. Sawada, K. Nakagawa, T. Okano, S. Yamada, H. Kakuta, A. Wada, Bioorg. Med. Chem. 2011, 19, 2939-2949.

[7] K. Tappe, P. Knochel, Tetrahedron: Asymmetry 2004, 15, 91-102.

[8] T.-Y. Dong, S. W. Chang, S.-F. Lin, M.-C. Lin, Y.-S. Wen, L. Lee Organometallics 2006, 25, 2018 2024.

[9] L. E. Wilson, C. Hassenrück, R. F. Winter, A. J. P. White, T. Albrecht, N. J. Long, Eur. J. Inorg. Chem. 2017, 496-504.

[10] C. Engtrakul, L. R. Sita, Organometallics 2008, 27, 927-937.

[11] A. Shafir, M. P. Power, G. D. Whitener, J. Arnold, Organometallics 2000, 19, 3978-3982.

[12] W. Yao, M. Chen, X. Liu, R. Jiang, S. Zhang, W. Chen, Catal. Sci. Technol. 2014, 4, 1726-1729.

[13] P. Srinivas, S. Prabhakar, F. Chevallier, E. Nassar, W. Erb, V. Dorcet, V. Jouikov, P. R. Krishna, F. Mongin, New. J. Chem. 2016, 40, 9441-9447.

[14] S. Dey, J. W. Quail, J. Müller, Organometallics 2015, 34, 3039-3046. 